\title{
CODEX MONACENSIS
}

n. 324 cum editione Ducangii collatus.

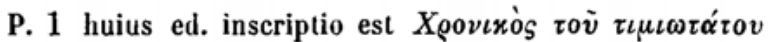

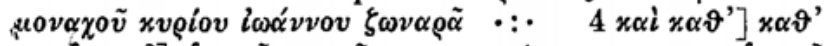

$\tilde{\varepsilon} \alpha v \tau o \tilde{v}] \tilde{\varepsilon} \alpha v \tau \tilde{\omega}$, et oiv margo r., i. e. manu rec. $\hat{\varepsilon} \alpha v \tau \tilde{\omega}$ etiam A, qui eandem constructionem infert vol. 1, p. 287, B́,

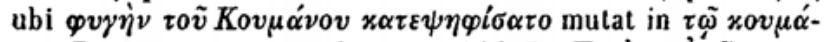
$\nu \omega$. Et sic omnes Annae Comn. p. 146, B: $\Sigma \iota \gamma \dot{\eta} v \alpha v \tau \tilde{\omega}$ x $\alpha \tau \alpha-$

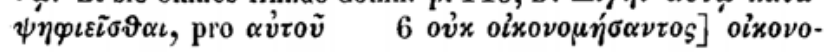
$\alpha$

$\mu \dot{\eta} \sigma \alpha \nu \tau$, sed os margo r. $7 \dot{\varepsilon} \pi \varepsilon \iota \delta \dot{\eta}] \dot{\varepsilon} \pi \varepsilon \dot{i} \delta \dot{\varepsilon}$, sed $\dot{\varepsilon} \pi \varepsilon \iota \delta \dot{\eta}$

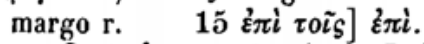

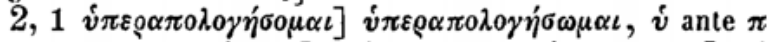

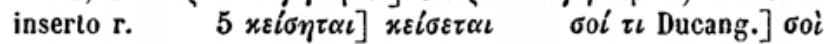
ut ceteri: quod notavi, alioqui mera Parisinae vitia omittens, ut p. 1, $4 \mu \varepsilon \varepsilon \tau \alpha \nu \alpha \sigma \tau \varepsilon \dot{v} \sigma 0 \nu \tau \alpha$ pro $\mu \varepsilon \tau \alpha \nu \alpha \sigma \tau \varepsilon v ́ \sigma \alpha \nu \tau \alpha \quad 8 \tau \alpha^{\prime} \tau \tau \varepsilon$ $\left.\not \lambda \lambda \alpha_{\S}\right]$ om. $\left.11 \sigma \tau \rho \alpha \tau 0 \pi \varepsilon \delta \varepsilon i \alpha_{\xi}\right] \sigma \tau \rho \alpha \tau 0 \pi \alpha \iota \delta \varepsilon i \alpha_{\S}$, sed $\varepsilon$ margo r. $\left.14 \alpha v^{\prime} \omega^{\prime} v \omega v\right] \alpha v^{\prime} \alpha i o v$, sed cum ductu super $\alpha i ́ o v$, quo alibi genitivus pluralis indicatur, ut in sequenti $\chi \rho \eta \mu \alpha ́ \tau$ pro

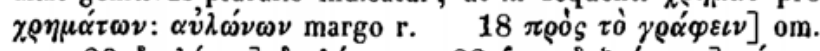

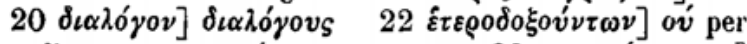
compendium pro ov, ov́v margo r. $23 \sigma v \gamma \gamma \varrho \alpha ́ q \omega \nu \tau \alpha \iota]$

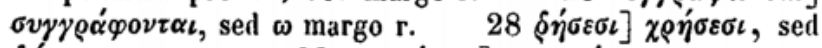
仓๊

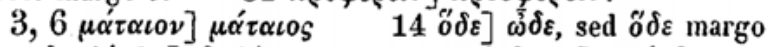

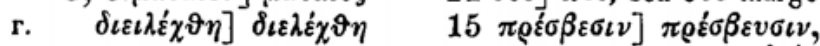

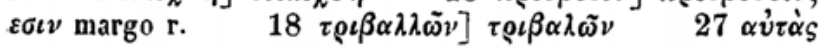




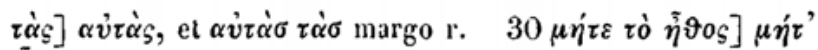
ทेंos.

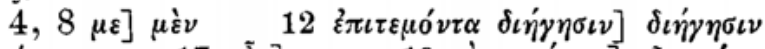

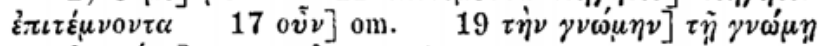
$\hat{\varrho} \alpha \sigma \tau \omega \nu \eta]$ erasum $\hat{\alpha} \alpha \sigma \tau \omega$ initio paginae, quippe scriptum iam v. ult. praecedentis $\left.\left.23 \delta \delta^{\prime} \mu \varepsilon\right] \delta \delta^{\prime} \gamma^{\prime} \mu \varepsilon \quad 27 \vartheta v \rho.\right]$ $\boldsymbol{\nabla} \eta \rho$ et $\boldsymbol{v}$ margo $\mathrm{r}$.

$5,7 x \lambda v \delta \omega \nu \iota \alpha] x \lambda v ́ \delta \omega \nu \alpha \quad z \pi i \pi \alpha \nu] \pi \tilde{\alpha} \nu$ unargo r. ad hunc versum, quod huc referendum 15 ov้ $\tau \varepsilon]$ ov́ $\tau \omega$, et

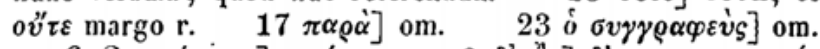

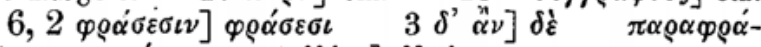

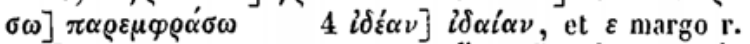

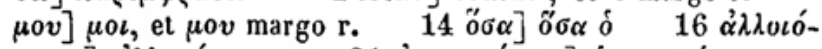

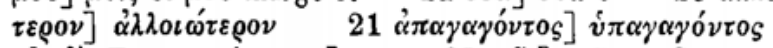

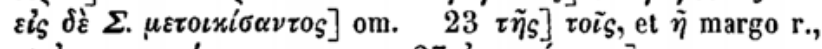

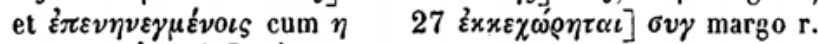

$28 \tau \dot{\eta} \nu \tau \tilde{\omega} \nu] \tau \dot{\eta} \nu$, non item 31, ut perhibet Pinderus, ubi sic alii.

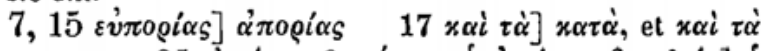

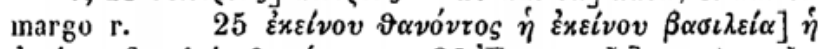

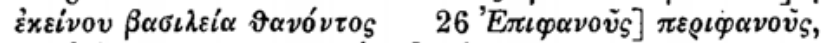

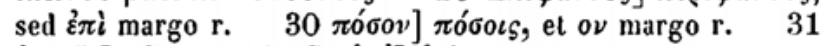

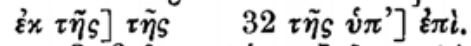

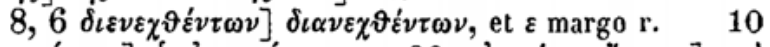

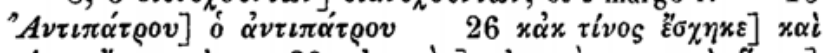

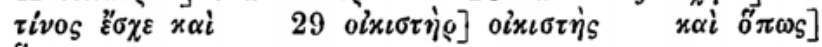
"̈ $\pi \omega$ s.

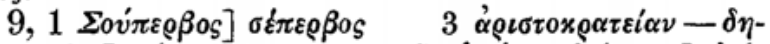

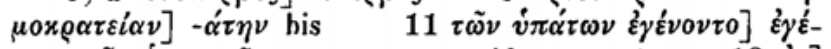

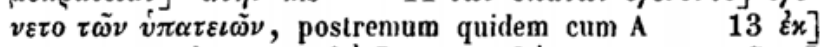

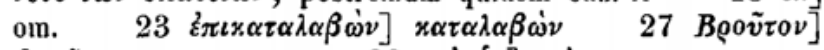

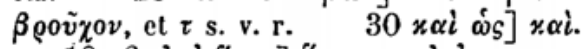

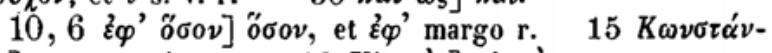

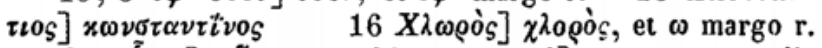

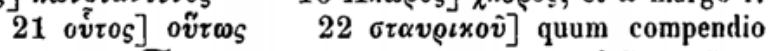

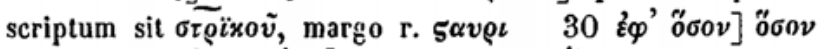

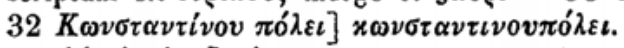

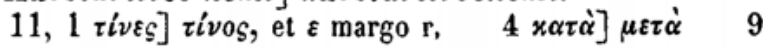


$\ddot{\alpha} \varrho \varrho \eta r o v$ vulgo additum] $\dot{\alpha} \varrho \chi \dot{\eta} v$, scriptum $\stackrel{\chi}{\alpha \varrho}$, et $\ddot{\alpha} \varrho \varrho \eta \tau o \nu$

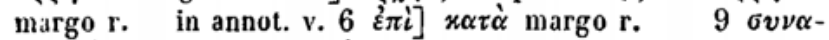
$\left.\gamma \alpha \lambda \lambda \omega^{\prime} u \varepsilon v o \nu\right] \sigma v \nu \alpha \gamma \alpha \lambda \lambda \mu^{\prime} \varepsilon v 0 \nu$, quod reponendum pro eo quod Pinderus intulit $\sigma v v \alpha \gamma \alpha \lambda \lambda \iota \omega^{\prime} \mu \varepsilon v o \nu$. Alterum ego tacito posueram at Stephanum $14 \chi \varrho \eta \sigma \alpha ́ \mu \varepsilon \nu 0 \varsigma] \chi \varrho \eta \sigma \alpha ́ \mu \varepsilon \nu 0 \nu$

$15 \tau \alpha \xi\llcorner\alpha \varrho \chi \alpha \rho] \tau \alpha \xi \iota \alpha \rho \chi \alpha_{S}$ recte, sed $\alpha^{\prime} \varrho \chi \alpha \sigma$ margo r. $\quad 17$ post illud $\varepsilon^{2} \nu \vartheta \mu \eta^{\prime} \sigma \varepsilon \omega \varsigma$, quo finitur appendix ab me eiecta, codex eodem versu continuo pergit his sex versibus, quos ego repetii ut sunt in illo divisi :

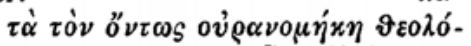

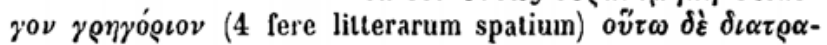
( 8 fere litt. spatium) $v 0 \tilde{v} \nu$ ( 6 fere litt. spatium) $\tau \alpha$ ( 8 fere litt. spatium)

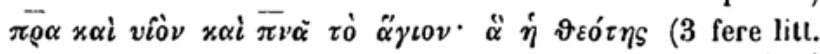

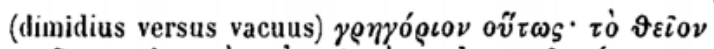
spatium)

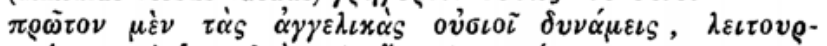

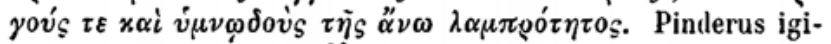
tur quod perhibet illud $\delta$, quod cum aliis libris duobus p. 12,

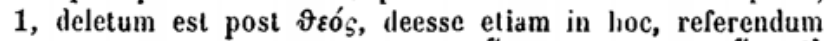
potius ad versum 7 , ubi hic codex ov́ $\tau \omega \subseteq$ tantum pro ov́ $\tau \omega \delta$ ' " $\chi 0 \nu$, illa vero verba $\vartheta \varepsilon \dot{S} \delta$ " है $\sigma \tau \iota \mu \dot{\varepsilon} \nu$ omnino non habet.

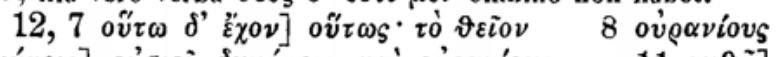

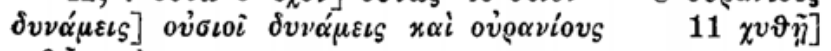
$\chi \varepsilon \vartheta \grave{\eta}$, sed $v$ margo r.

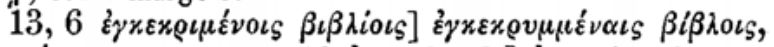

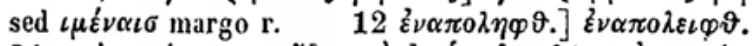

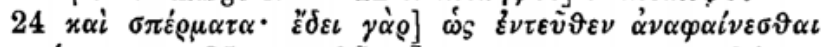

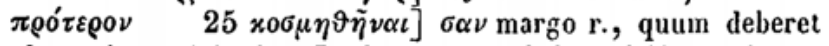
$\varepsilon \hat{i} \sigma \alpha \nu$ : ita accipiendum Pinderi , $\varkappa 0 \sigma \mu \eta \vartheta \varepsilon \tilde{\sigma} \sigma \alpha \nu$ C."

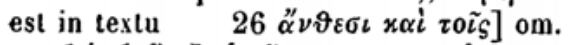

$\left.14,1 i^{\prime \prime} \nu \alpha\right] \omega_{S} \tilde{i}^{\prime \prime} \nu \alpha$, sed infra $\dot{\omega}_{S}$ lineola r. $3 x \alpha i \sigma \eta$ $\mu \varepsilon \tilde{i} \alpha]$ pro his lacunam totidem litterarum, unde post $\pi \alpha \rho \varepsilon$ -

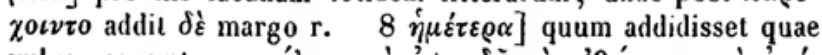

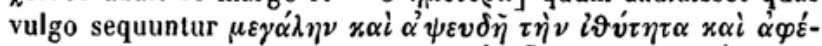
$\lambda \varepsilon \iota \alpha \nu$, pro sequentibus $\left.\tau \tilde{\eta} \pi \dot{\varepsilon} \mu \pi \tau \eta \delta^{\prime} \alpha \tilde{v} \vartheta \iota \varsigma-17 \tau \dot{\eta} \nu \gamma \tilde{\eta} \nu\right]$

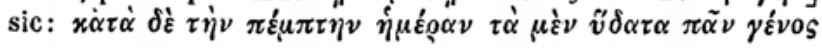




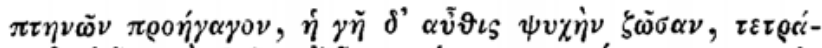

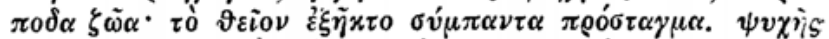

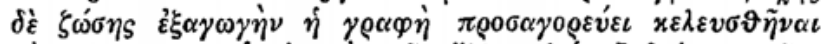

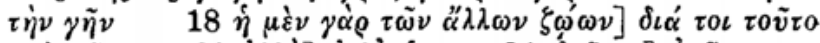

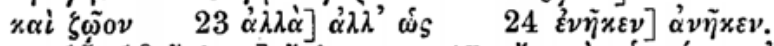

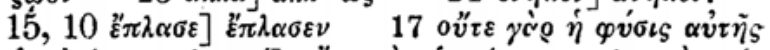

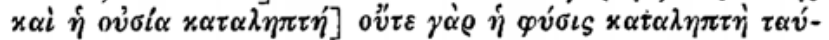

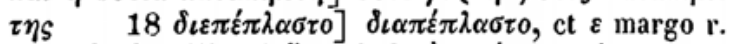

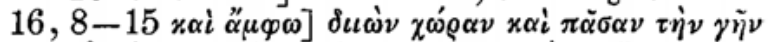

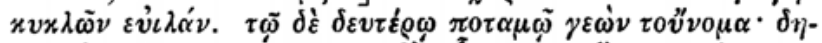

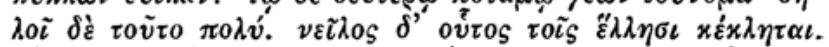

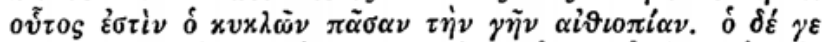

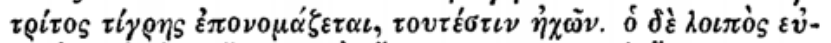

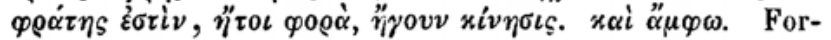

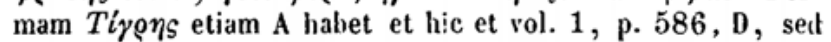

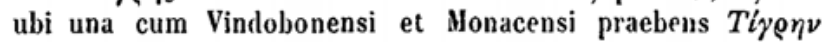
suspectum reddit quod hic quidem est etian in plerisque lo-

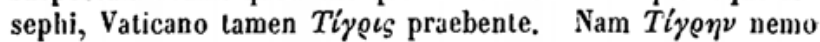

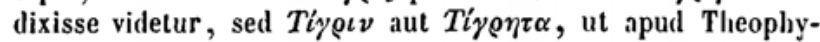
lactum quoque Hist. p. 25, C, et Cedrenum p. $396, \mathrm{~B}$, pro $T^{\prime} \gamma \varrho \eta \nu$ restituendum sit $T^{\prime} \gamma \varrho \iota \nu$, ut uterque semper dicit

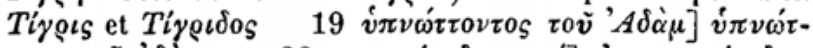

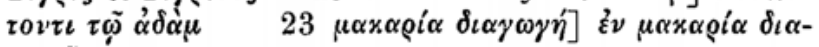
$\gamma \omega \gamma \tilde{\eta}$.

17, 11 ' $A \delta \dot{\alpha} \mu$ i $\delta \pi \dot{\alpha} \gamma \varepsilon \iota]$ vं $\pi \dot{\alpha} \gamma \varepsilon \iota v$, deleto $\nu$ ead. m. rec. quae omissum ' $A \delta \dot{\alpha} \mu$ supplevit.

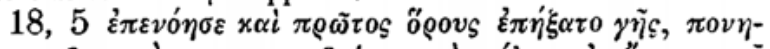

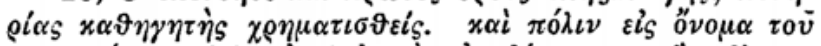

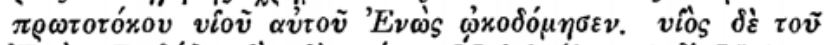

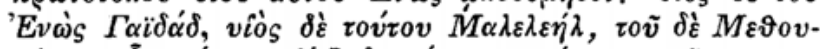

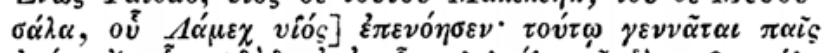

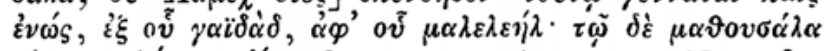
$\tau i \hbar \tau \varepsilon \tau \alpha \iota$ 수 $\mu \varepsilon \chi$ viós. In marg. asteriscus r. $13 \pi \varrho \circ \beta \alpha$ -

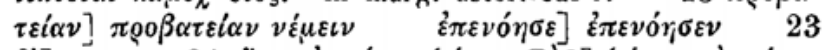

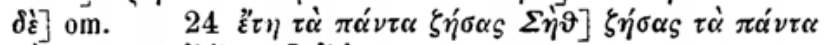

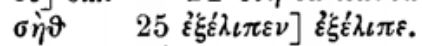

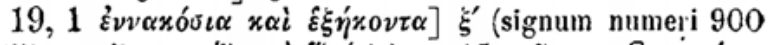

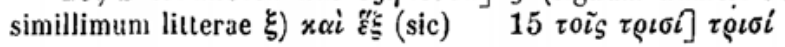


$22 \dot{\varepsilon} x \tau \tilde{\omega} \nu] \tau \tilde{\omega} \nu \dot{\varepsilon} \varkappa$

$29 \pi \varrho 0 \sigma \omega ́ \chi \vartheta \iota \sigma \varepsilon] \pi \varrho 0 \sigma \omega ́ \chi \vartheta \iota \sigma \alpha \nu$ perspicue.

$20,1 N \tilde{\omega} \varepsilon] \nu \tilde{\omega} \varepsilon \delta \dot{\varepsilon}$

$\left.2 \delta \iota^{\prime} \dot{\varepsilon} \pi \circ \mu \beta \rho i \alpha_{S}\right]$ om.

6

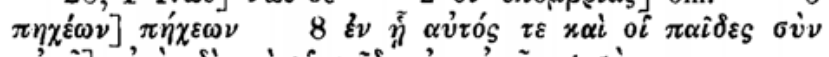

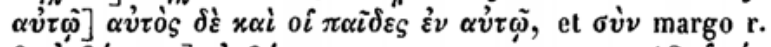

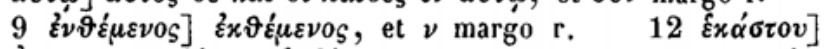

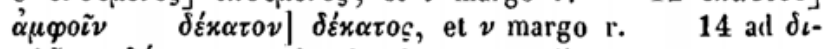
$\pi \lambda \tilde{\eta} v-\delta v_{0}$, quae vulgo legebantur pro is quae ego ex me-

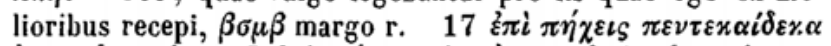

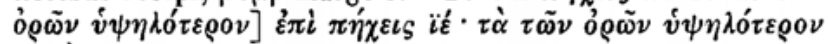
ชอ

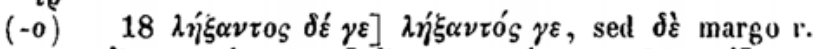

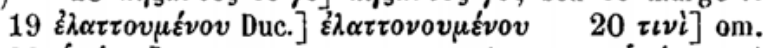
$23 \dot{\eta} \mu \varepsilon \alpha_{\varsigma}$ ] asteriscus r. super $\sigma$ (pro quo $\dot{\eta} \mu \varepsilon \dot{\varepsilon} \alpha \nu$ A)

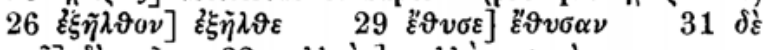

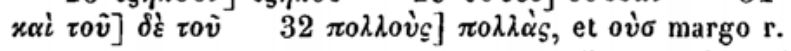

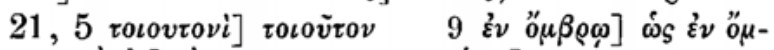

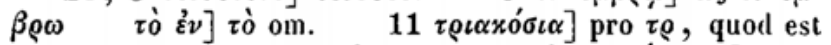
in rasura, haud llubie pr. $\delta$, ut $\mathbf{A} \quad \alpha \alpha i \pi \varepsilon v \tau \eta \dot{\eta} \kappa o v \tau \alpha] \pi \varepsilon v-$

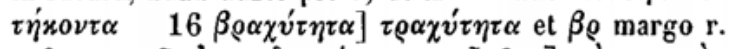

19

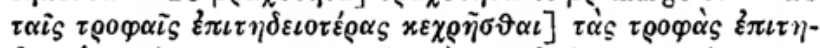
$\delta \varepsilon \iota \tau t \rho \alpha_{S}$ (pr. - $\rho 0 v \varsigma$, ut videtur) $x \varepsilon \chi \rho \tilde{\eta} \sigma \vartheta \alpha \iota$, et $\tau \alpha i_{S}$ margo $r$., sequentibus syllabis $-\alpha_{S}$ lineola notatis $\left.21 \delta i \dot{\alpha}\right]$ on., sed addit margo r. $22 \tau \varepsilon x \alpha i] x \alpha i \quad 26 \tau 0 \tilde{v} N \omega \tilde{\varepsilon}]_{\text {e }} \nu \tilde{\omega} \varepsilon$.

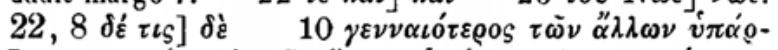

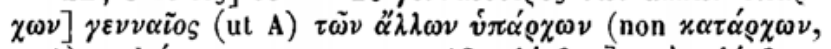

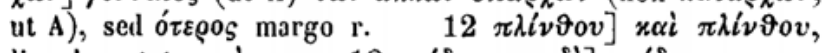
lineola notato $x \alpha i \mathrm{r} . \quad 18 \sigma x i \delta \nu \alpha \nu \tau \alpha \iota \quad \delta \dot{\xi}] \quad \sigma x i \delta \nu \alpha \nu \tau \alpha \iota$

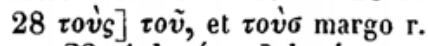

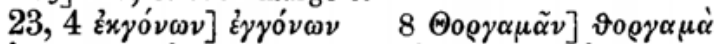

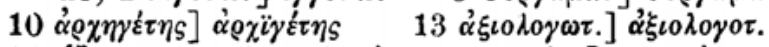

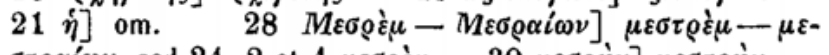

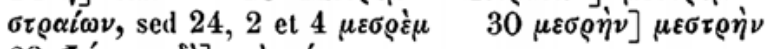
32 Фó $\nu \tau \eta \nu ~ \delta \dot{\varepsilon}] x \alpha i$ ตó $\nu \tau \eta v$.

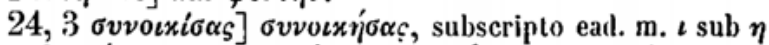

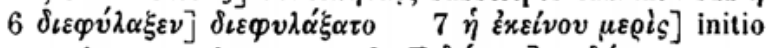
versus omissa cum lacuna $8 \Sigma\left\llcorner\delta \omega^{v} \nu \iota_{s}\right] \sigma \iota \delta^{\alpha} \nu \iota 0_{S}$, et $\omega$

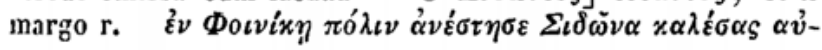




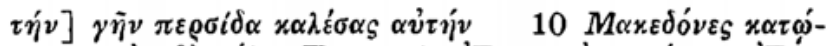

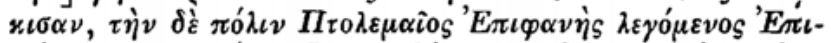

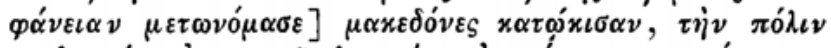

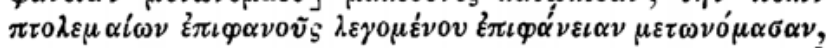
confusa vulgata cum ea quam A praebuil scriptura 14 roṽ]

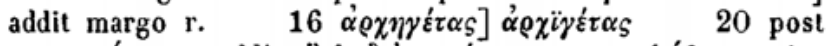

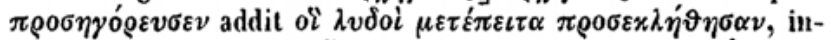

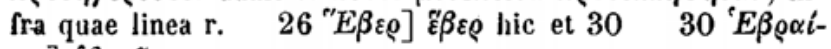
$\omega \nu] \varepsilon \beta \rho \alpha \tilde{\imath} \boldsymbol{~} \iota$.

\section{o}

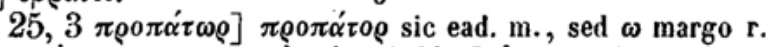

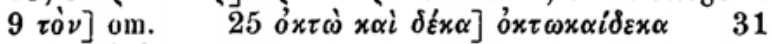

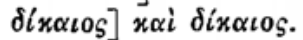

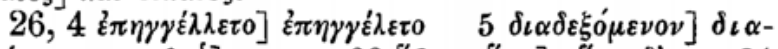

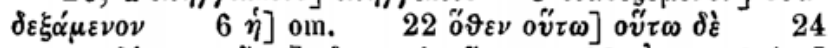

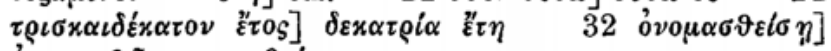

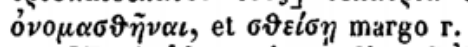

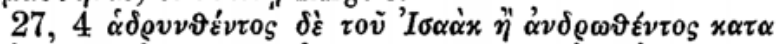

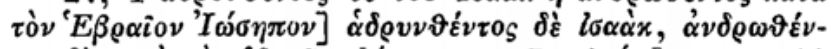

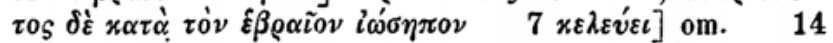

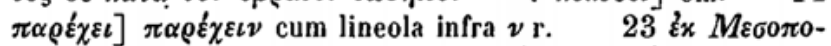

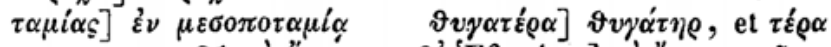

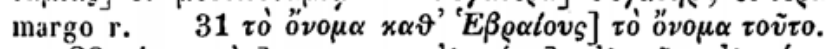

$28,4 \varphi \alpha \gamma \dot{\nu} v]$ om.

$5 \alpha \pi 0 \vartheta \alpha \nu \varepsilon i \nu] \vartheta \alpha \nu \varepsilon i \nu, 12 \pi \alpha \rho \varepsilon \sigma x \varepsilon v \alpha \sigma \mu \varepsilon \dot{v} \nu \omega \nu]-v 0 v$, sed $\omega \nu$ margo r. $24 \delta \dot{\varepsilon}] \delta^{\prime}$.

$29,8 K \alpha \varrho \varrho \dot{\alpha} \nu] \chi \alpha \rho \dot{\alpha} \nu$, sed $x \alpha \varrho \oint \dot{\alpha} \nu$ punctis infra positis

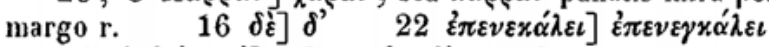
$30 \tau \tilde{\omega} \alpha \nu \delta \rho i \quad x \alpha i] \tau \tilde{\omega} \pi \alpha \tilde{i} \delta \alpha \delta \dot{\varepsilon} \gamma \varepsilon \nu \nu \tilde{\eta} \sigma \alpha i$, sic prorsus $\left.32 \delta \eta \lambda_{0} i \bar{\imath}\right] \lambda \varepsilon \varepsilon \varepsilon \tau \alpha \iota:$ v. p. $30,15$.

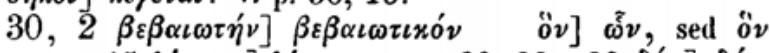

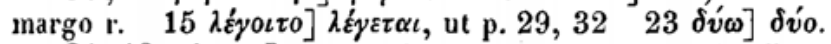
$31,13$ yiver $\alpha \iota]$ om., sed addit margo r.: unde intelligitur quomodo $x \alpha i$ ó $\eta^{\prime} \rho \varepsilon v \nu \alpha$, quod ex A recepi, primum in

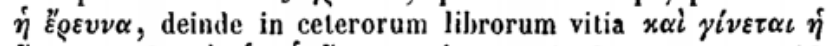

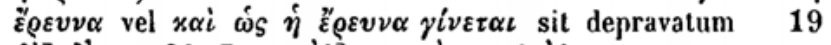
$\left.\delta \dot{\varepsilon}] \delta^{\prime} \quad 29 \Phi \alpha \nu 0 v \dot{\eta} \lambda\right]$ $\varphi \alpha \nu o v \chi$, sed $\dot{\eta} \lambda$ margo r.

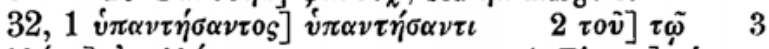

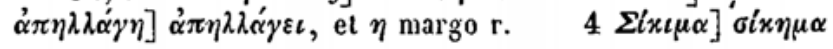




\section{$8 \Sigma \iota x \iota \mu \iota \tau \dot{\omega} \nu] \sigma \iota x \eta j \mu \omega \nu \quad 9 \Delta \varepsilon i v \alpha \nu] \delta \varepsilon i v \alpha$ hic et 17}

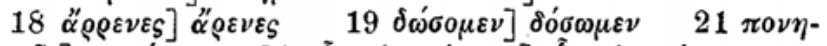

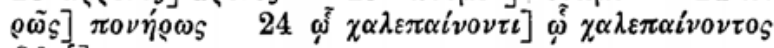
26 o $] \mathrm{om}$.

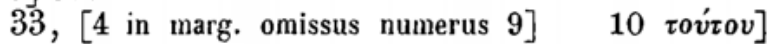

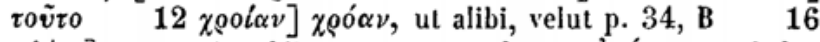
$\left.\pi \lambda \varepsilon_{0} v\right]$ om. ut A, aldit margo r., sed post $\eta^{\prime} \gamma \alpha \dot{\alpha} \alpha$ non habet $\mu \tilde{\alpha} \lambda \lambda o \nu$, ut A, quocum non convenit Iosephus, qui $\pi \lambda \hat{\varepsilon} o v$, non $\left.\mu \tilde{\alpha} \lambda_{0} \nu 18 \delta_{\dot{\varepsilon}}\right] \delta$ '.

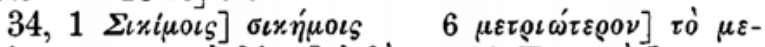

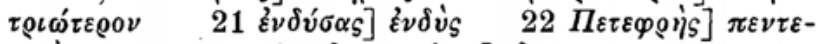
$\varphi \rho \dot{\eta}_{s}$ hic et $\left.2626 \delta_{\iota \alpha \tau \varepsilon \vartheta \varepsilon i \sigma \eta}\right] \delta \iota \alpha \tau \varepsilon \vartheta \varepsilon \tilde{\sigma} \sigma \alpha \quad \pi \rho \circ \sigma \alpha-$

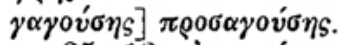

$\left.35,13 \varepsilon \dot{v} \pi \rho \alpha \gamma \eta^{\prime} \sigma \alpha \tau \alpha\right] \varepsilon \dot{v} \pi \rho \alpha \gamma \dot{\eta} \sigma \alpha \nu \tau 0 \varsigma$ 17. $\left.\tau 0 v i \omega\right]$

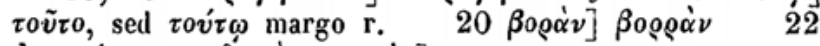

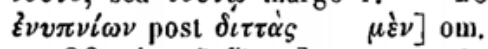

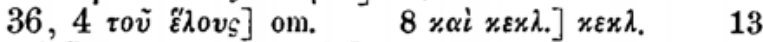

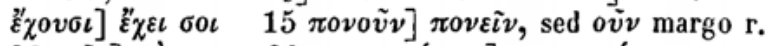

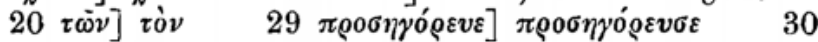

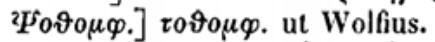

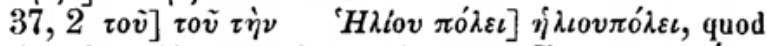
praeferendum videtur apud recentiores, ut $K \omega \nu \sigma \tau \alpha \nu \tau \iota \nu 0 v ́ \pi 0-$ $\lambda_{\iota \varsigma}$ el alia $\left.5 \tau \dot{\eta} \nu-\dot{\varepsilon} \lambda \varepsilon v \vartheta \varepsilon \rho i \alpha \nu\right] \tau \dot{\alpha}-\varepsilon \dot{\lambda} \varepsilon \varepsilon \dot{v} \vartheta \varepsilon \rho \alpha \quad 10$

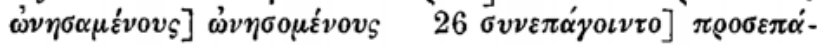
joเvนo.

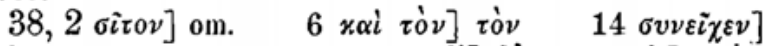

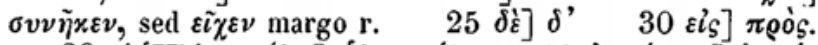

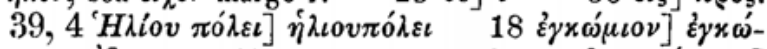
$\mu \iota \alpha \mu \grave{\eta}]$ om., addit margo r. $21 \sigma v \nu \delta \iota \alpha \iota \rho o v \mu \varepsilon v o \iota]$ $\sigma v \nu \delta\llcorner\alpha \iota \rho 0 v \mu \varepsilon v \omega$, corr. margo $\mathrm{r}$.

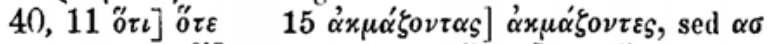

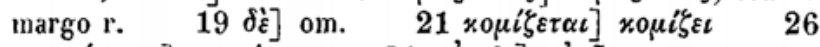

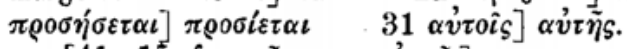

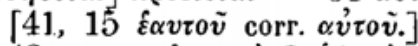

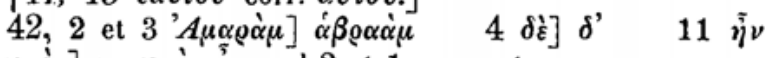

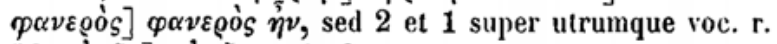
$23 \alpha \dot{v} \tau \tilde{\omega} \nu] \alpha \dot{v} \tau \tilde{\omega} \nu$, sed ov margo r.

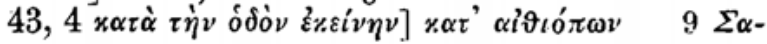




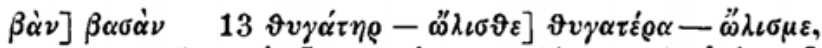

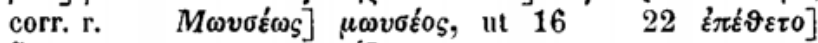
ह้งยน , sed corr. r. 30 ó] om.

$44,2 \dddot{\eta}]$ om., addit margo r. $6 \tau \varepsilon]$ on. $10 \dot{\varepsilon} \pi \eta \gamma$ -

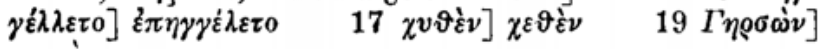
$\gamma \eta \varrho^{\alpha}$, quod villetur esse $\gamma \eta \alpha^{\prime} \nu$, ut $\Gamma \eta \varrho \sigma \dot{\alpha} \mu$ LXX 28 $\varepsilon \pi \alpha 0 \iota \delta \tilde{\omega} \nu] \lambda \alpha 0 \iota \delta \tilde{\omega} \nu \quad 29 M \omega v \sigma \tilde{\eta} \varsigma] \mu \omega \sigma \tilde{\eta}_{S} \quad 32 x \alpha \tau \dot{\eta}^{\prime}$

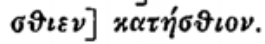

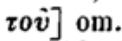

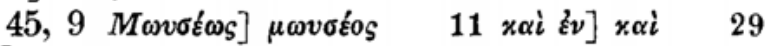

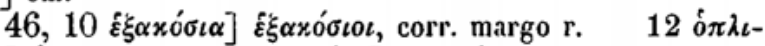

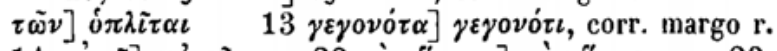

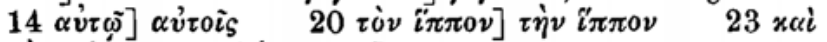
$\tau \dot{\alpha} \tau o v ́ r o v-25 \dot{\xi} v \dot{\mu} \pi \alpha \nu \tau \varepsilon \xi]$ om.

47, $8 M \dot{\alpha} \varrho$ et $9 \mu \dot{\alpha} \varrho] \mu \dot{\alpha} \nu$ et $\mu \dot{\alpha} \nu \quad 14 \gamma \lambda v x \alpha \nu \vartheta \tilde{\eta} \nu \alpha \iota]$

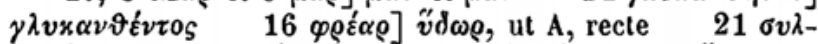

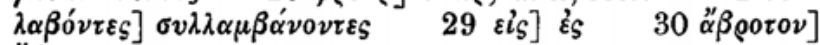
$\alpha \beta \varrho \omega \tau o v$, ut ego scripseram: $\alpha$ et proxima littera in rasura: $\beta$ margo r.

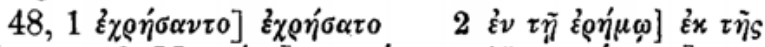

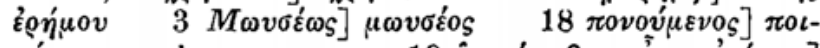

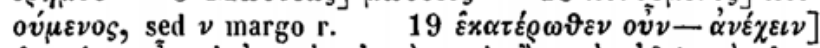

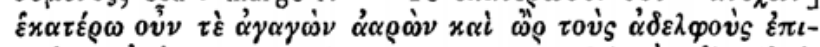
$\sigma \tau \eta^{\prime} \sigma \alpha \mathcal{\alpha} \alpha \varepsilon^{\prime} \chi \varepsilon \iota \nu$, cum + in marg. r.

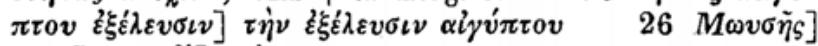

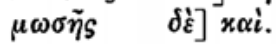

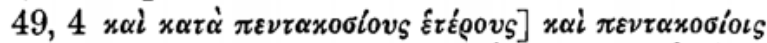

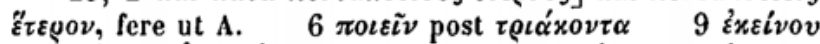

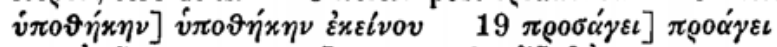
$25 \alpha v \tau \tilde{\omega}$ vulgo additum] om. $28 \xi \xi \xi] \delta$ '.

$50,7 \sigma 0 v]$ oin. ut $\mathbf{A}$, recte $\dot{\alpha} \varphi i \eta \varsigma] \dot{\alpha} \varphi \varepsilon i s$, et $i \eta \varsigma$

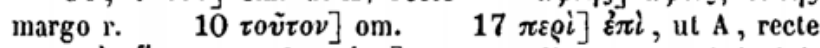
$x \alpha i$ oṽ $\left.\tau \omega_{\varsigma}-x \alpha \vartheta \alpha \varrho \sigma i \omega \nu\right]$ om., medio versu qui incipit $\varepsilon \pi \dot{\imath} \tau \dot{\eta} \nu$, desinit $\kappa \alpha \dot{\zeta} \zeta \omega \omega \nu$, nonnisi dimidia eius parte vacua $\left.\begin{array}{lll}\text { relicta } & 19 & \tau \varepsilon\end{array}\right]$ om. ut $\mathrm{A}$, recte 24 órı] om. 32 $\left.\tau \tilde{\eta}_{S} M \alpha \varrho \iota \dot{\alpha} \mu\right] \mu \alpha \varrho \iota \dot{\alpha} \mu$. 


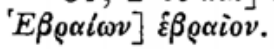

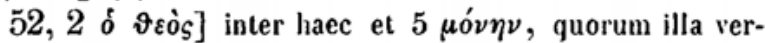
sum finiunt, hoc inchoat, integer versus vacuus relictus 7 $\pi \dot{\alpha} \nu v] \pi \alpha^{\prime} \lambda \iota v$ perspicue $\left.\dot{\omega}_{\zeta}\right]$ om. et $\tilde{\sigma} \sigma \tau \varepsilon$ margo r. 8 $\left.\left.M \omega \nu \sigma \tilde{\eta}_{S}\right] \mu \omega \sigma \tilde{\eta}_{S} \quad 21 \dot{v} \varphi \eta \gamma \omega \bar{\nu} \tau \alpha L\right] \dot{v} \varphi \eta \mu \tilde{\eta} \nu \tau \alpha \iota$, et $\dot{v} \varphi \eta$ -

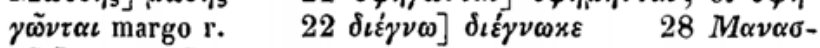
$\sigma \tilde{\eta} \nu] \mu \alpha \nu \alpha \sigma \sigma \tilde{\eta}$.

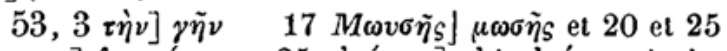

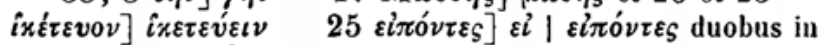

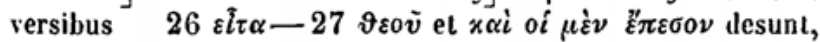
vacuis spatiis in versuum initiis, qui incipiunt $\alpha \alpha i \quad \pi \rho 0 \sigma \beta \alpha$ -

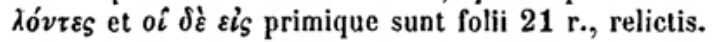

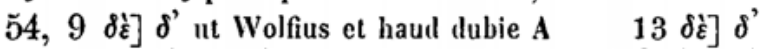

$\left.15 M \omega v \sigma \tilde{\eta}_{S}\right] \mu \omega \sigma \tilde{\eta}_{S}$, ut $\left.25,27,2824 \dot{\varepsilon} \pi \eta \eta^{\prime} \nu \eta \sigma \varepsilon\right]$

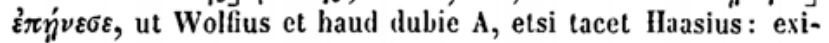
mendum igitur hoc iis de quilus dixi praef. vol. 1, p. VI.

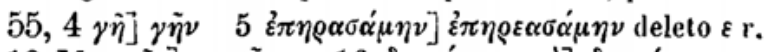

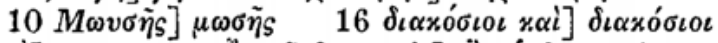

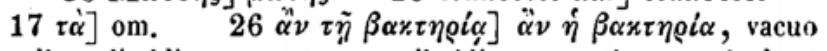
relicto dimidio versu et prope dimidio sequenti, quem inchoat $\lambda \varepsilon v i \tau \eta v$.

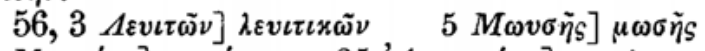

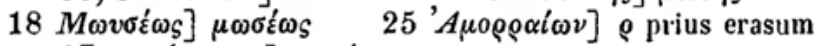

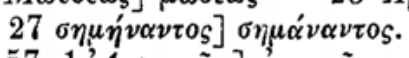

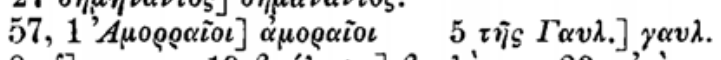

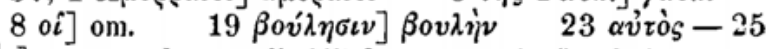
$\dddot{o} \alpha \nu]$ om. cum lacuna dinidii fere versus in fine huius et sequentis initio 26 ö oos] rò ö oos.

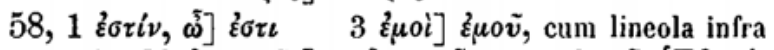

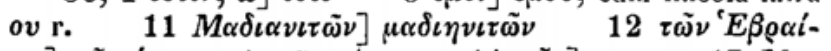
$\omega \nu] \tau \tilde{\omega} \nu \varepsilon_{\varepsilon}^{\prime} \omega \nu$, ut A $\left.\tau \tilde{\omega} \nu \nu \bar{\varepsilon} \omega \nu 14 \tau \tilde{\varepsilon}\right]$ om. $17 M \alpha-$

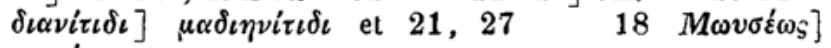
$\mu \omega \sigma \varepsilon \omega \varsigma$.

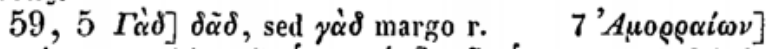

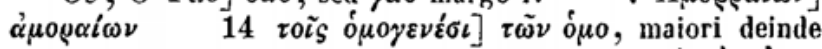
versus parte vacua relicta et proximo incipiente $\alpha \alpha \dot{i} \tau \dot{\eta} \nu \dot{\alpha} \mu 0-$ 


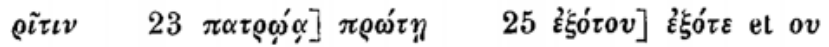
margo $r$.

$60,10 \pi \rho 0 \varepsilon v \varepsilon \gamma x \varepsilon \tilde{\nu} \nu] \pi \rho 0 \sigma \varepsilon \nu \varepsilon \gamma x \varepsilon i \nu \quad 15 N \alpha \beta \dot{\alpha} \nu] \nu \alpha-$ $\left.\beta \alpha \tilde{v} 19 \delta^{\prime}\right]$ pro hoc $\delta \dot{\varepsilon}$ anguste insertum ead. $\mathrm{m}$.

$61,8 \varepsilon \pi \circ \mu . \pi . \mu$.] in fine versus cum vacuo spatio omissa supplevit r. $9 \dot{0} \pi \lambda i \tau \alpha \iota \varsigma] \dot{\omega} \pi \lambda \iota \tau \tilde{\omega} \nu, \omega$ mutato in marg. in o r. $\left.\quad 13 \delta \delta^{\prime}\right] \delta^{\prime}$, ut Wolfius, quol dedi: v. conti-

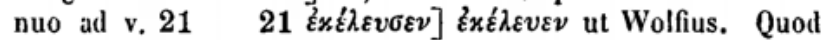
quum etiam apud losephum 5, 1, 2 praebuerint libri, Parisinae vitium felellisse videtur Haasium et recipiendum esse

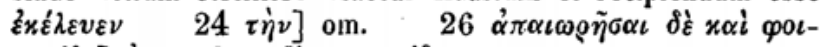

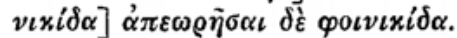

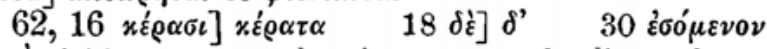
- $\gamma \dot{\alpha} \varrho$ initio versus suppleta in vacuo quod relictum fuerat spatio $\mathrm{r}$.

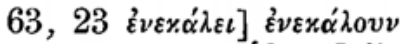
haud dubie $\mathbf{A}$

$\delta \dot{\varepsilon}] \delta$ ' ut Wolfius et

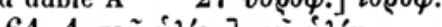
haud dubie A $\left.10 \tau \dot{\omega} \nu] \tau \dot{\nu} \nu \quad 26 \tau \dot{\nu} \nu \lambda \alpha \dot{\nu} \nu \varepsilon i_{S} \Sigma \eta \lambda \omega \mu\right]$

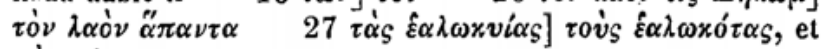
$\tau \dot{\alpha} \alpha_{S} v i \alpha_{S}$ margo $\mathrm{r}$.

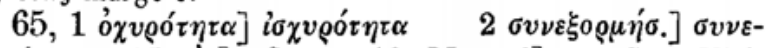

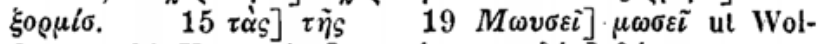
fius $\left.28 \mathrm{X} \alpha \nu \alpha \nu \alpha i o \iota]^{\prime} \chi^{\alpha \nu \alpha i o \iota s} \delta v^{\prime} \omega\right] \delta v^{\prime}$ ut ego.

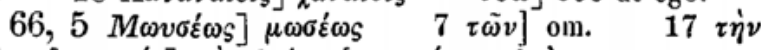

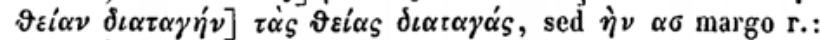
nam $\gamma \dot{\alpha} s$ compendio scriptum non animadvertit corrector.

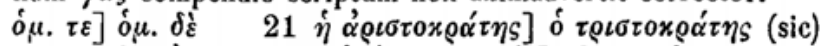
$23 \delta \varepsilon \iota \nu \dot{\eta}]$ om. $24 \dot{\alpha} \nu \grave{\eta} \rho]$ oin. $\left.\varepsilon^{2} x\right] \varepsilon i \xi$, sed हैx margo r. $67,5 \tau \tilde{\eta}$ ante $\lambda \dot{v} \pi \eta$ s. v. ead. m. $7 \tau \dot{\eta} \nu] \alpha \dot{v} \tau \dot{\eta} \nu$

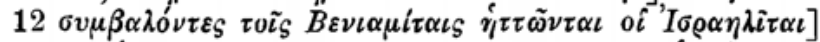

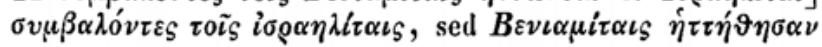
margo $r$.

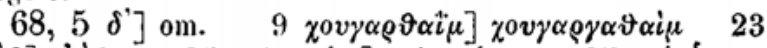

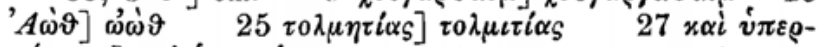

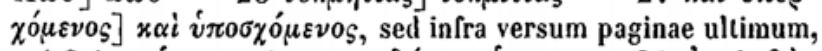

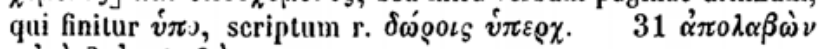
$\alpha \dot{v} \tilde{o} \nu] \dot{\alpha} \pi 0 \lambda \alpha \beta \dot{\omega} \nu$. 
69, $1 \xi \iota \varphi \iota \delta i \omega] \xi \iota \varphi i \delta \iota$ margo r. $14 \tau \tilde{\omega} \nu] \tau \tilde{\eta} \mathrm{s}$ volueram, ut dixi praef. vol. $1, \mathrm{p}$. V.

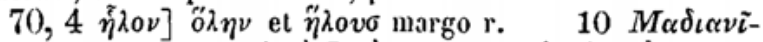
$\tau \alpha \iota] \mu \alpha \delta \iota \eta v i \tau \alpha \iota \quad 14 \tau \dot{o} \nu] \tau \dot{\eta} \nu 18 \alpha \dot{v} \tau \sigma \dot{v}] \alpha \dot{v} \tau \tilde{\omega}$ $\tau \hat{j}] \tau \tilde{\eta}$ recte, ut viletur, quum apud Iosephum hoc quoque sit 5, 6, 2, ut $\tau \dot{\eta} v$ paullo ante v. 14 ex A recepi, etsi Zonaras in talibus sibi non constat $21 \mu \varepsilon \tau \alpha v \alpha \sigma \tau \tilde{\eta} v \alpha \iota] \mu \varepsilon \tau \alpha-$ $\sigma \tau \tilde{\eta} \nu \alpha \iota$ $31 \dot{\varepsilon} \pi i \dot{i o \nu}] \dot{\varepsilon} \pi i$.

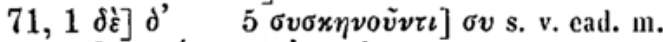

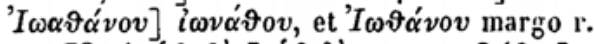

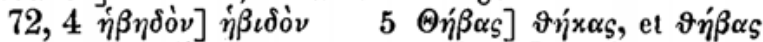

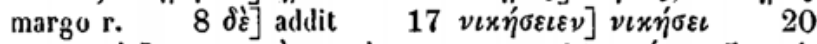
$\left.\mu 0 v 0 \gamma \varepsilon v \dot{\varepsilon}_{S}\right] \mu 0 \nu 0 \gamma \varepsilon v \grave{\eta}_{S}$, et $\dot{\varepsilon}$ margo r. $\left.21 \psi \varepsilon \varepsilon \dot{v} \sigma \tau \alpha \iota\right] \psi \varepsilon \dot{v}-$

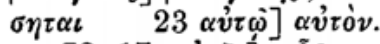

$73,17 \alpha v i \tilde{\eta} \varsigma$ ] $\alpha v^{*} \vartheta \iota \varsigma$.

$74,3 \delta \dot{\varepsilon}] \delta^{\prime}$ ut Wolfius et ego $\left.\Pi \alpha \lambda \alpha \iota \sigma \tau \iota \nu \omega ั \nu\right] \pi \alpha-$ $\lambda \alpha \iota \sigma \tau \eta \nu \tilde{\omega} \nu$

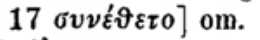
31 ๕̆] ò.

$75,17 \delta \dot{\varepsilon}] \delta^{\prime}$ ut Wolfius et ego $\left.20 \pi \eta \gamma \dot{\eta} \nu\right] \delta \rho o^{-}$

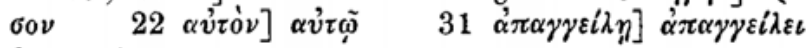
fere ut $\mathrm{A}$.

$76,8 x \alpha i]$ om. $\quad 17 \tau \rho \iota \sigma \chi \imath \lambda i \omega \nu] \tau \rho \iota \sigma \chi \iota \quad 20 \pi \alpha \rho \dot{\alpha}]$

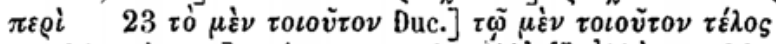

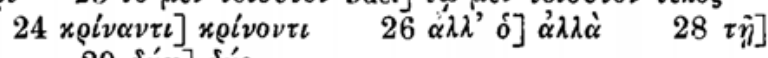
om. $\left.29 \delta v^{\prime} \omega\right] \delta v_{0}$.

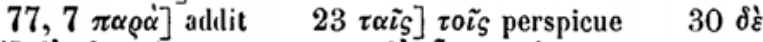
$\pi \varepsilon \rho i] \delta^{\prime} \varepsilon i \pi \varepsilon \rho i$, fere ut A, qui $\delta^{\prime} \varepsilon i \pi \tau \varepsilon \pi \varepsilon \rho i$, sed lineola sul, $\varepsilon i \pi \varepsilon \rho i$ et + margo $\mathbf{r}$.

$78,7$ ov $\chi]$ ovx

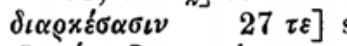
$\Phi \varepsilon v \alpha \nu v \alpha] \varphi \varepsilon v \nu \alpha ́ v \alpha$

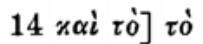

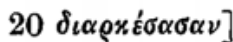
supra v. ead. $m$. $\quad 30$ et 79,1 $32 \Sigma \eta \lambda \dot{\omega} v] \sigma \eta \lambda \dot{\omega} \mu$.

$79,6 \delta \delta] \delta^{\prime}$ ut IVolfius et ego, etsi all $\delta \dot{\varepsilon}$ tacet Haasius

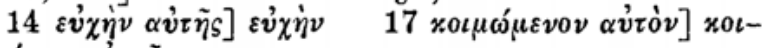

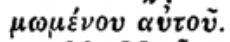

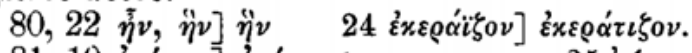

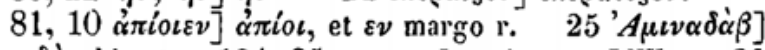
$\dot{\alpha} \mu \iota \nu \alpha \delta \dot{\alpha} \mu$ hic et p. 104, 25, contra Iosephum et LXX 29

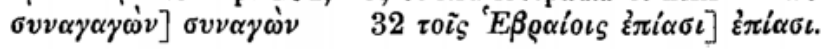


$82,8 \sigma \varepsilon \iota \sigma \mu \omega]$ ] $\sigma \varepsilon \iota \sigma \mu 0 \tilde{v} \quad 10 \delta \varepsilon \iota \lambda i \alpha \nu] \delta o v \lambda$. pr. scri-

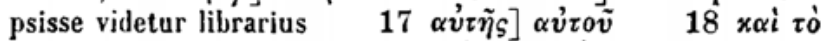

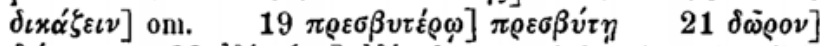

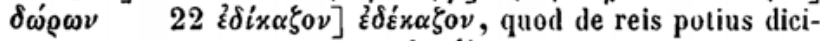
tur. Eliam infra p. $85,10 \delta \ddot{i x} \alpha_{s}^{\prime} \varepsilon t \nu$ pr. per $\varepsilon$ videri polest scriptum. terum.

83, $\left.12 \pi \rho \dot{o}_{S} \tau \dot{v} \nu \Sigma \alpha \mu o v \dot{\eta} \lambda\right]$ bis, sed rubro deletum al-

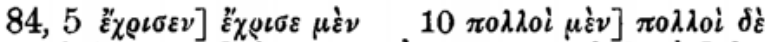

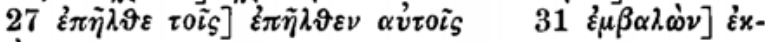
$\beta \alpha \lambda \omega \nu$, sed $\psi$ margo r.

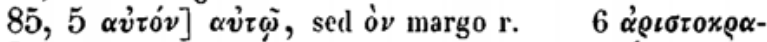

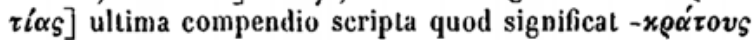

$\left.\left.M \omega v \sigma \omega_{S}\right] \mu \omega v \sigma_{\varepsilon} o_{j}^{\prime} \quad 10 \pi \varepsilon \rho i\right]$ om. $\left.26 \delta \iota \alpha \nu \alpha \sigma \tau \tilde{\eta} \nu \alpha \iota\right]$ $\delta \iota \alpha \sigma \tau \tilde{\eta} \nu \alpha \iota$, sed $\alpha \nu \alpha$ margo r.

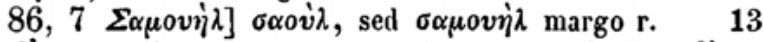
$\delta \dot{\varepsilon}] \delta$ ' ut Wolfius et ego, et saepius supra, ubi Par. $\delta \dot{\varepsilon}$ ta-

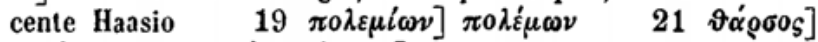

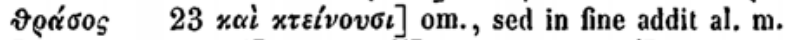

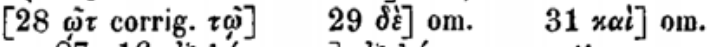

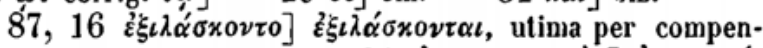
dium, quod est $\alpha \iota$, non $024 \dot{\alpha} \varrho \chi \iota \sigma \rho \alpha \tau \eta \gamma \dot{0} \nu] \dot{\alpha} \rho \iota \sigma \tau \rho \alpha^{\prime}-$ $\tau \eta \gamma o v$ ut ego.

$88,7 \mu \varepsilon \tau \alpha \mu \varepsilon \lambda \eta \tilde{\sigma} \alpha \iota] \mu \varepsilon \tau \alpha \mu \varepsilon \lambda \tilde{\eta} \sigma \vartheta \alpha \iota \quad 8 \delta \dot{\varepsilon}] \delta^{\prime}$.

$\left.\left.89,13 \varepsilon^{\xi} \varphi \eta^{\prime} \lambda \alpha \tau 0\right] \varepsilon \varphi \dot{\eta}^{\prime} \lambda \lambda \alpha \tau 0 \quad 16 \tau \iota \nu \alpha\right]$ om. $\pi \eta \chi \dot{\varepsilon} \omega \nu$ Duc.] $\pi \eta \dot{\eta} \chi \omega \nu$.

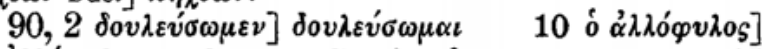
oi $\dot{\alpha} \lambda \lambda_{o} \varphi v \lambda o \iota$, sed notato lineola oi et $\sigma$ margo r. 15

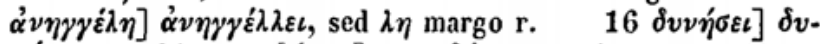
$\left.\nu \eta^{\prime} \sigma y .129 \sigma \varphi \varepsilon \nu \delta o ́ v \eta \nu\right] \sigma \varphi \varepsilon v \delta \delta^{\prime} \nu \alpha$, qua forma usi videtur

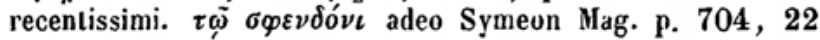
ell. Bonn.

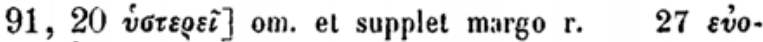

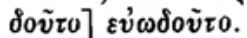

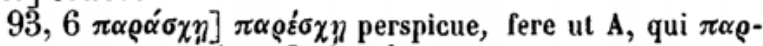

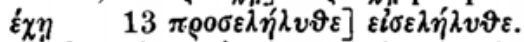

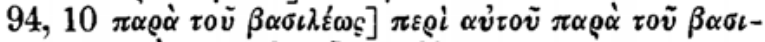

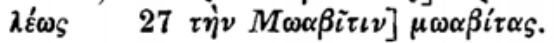




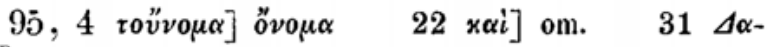
$\beta i \delta]$ om.

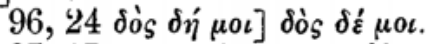

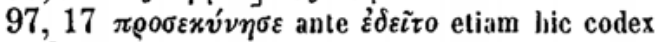
$\delta \varepsilon i \mu \alpha \tau \iota] \delta \varepsilon i \gamma \mu \alpha \tau \iota$, sed cum lineola infra $\gamma \mathrm{r}$. $27 \mu \nu \omega \dot{\mu} \varepsilon$ $\nu 0 \nu] \mu \nu \omega \mu^{\prime} v \nu_{\S}$ cum ultimae compendio, quod os est, non $0 v$.

$98,2 \tau \varepsilon \tau \rho \alpha x_{0 \sigma i \omega \nu]} \varepsilon \xi \alpha x o \sigma i \omega \nu$, et $\tau \varepsilon \tau \rho \alpha x$. r. $\quad 11$

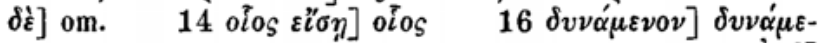
vos ultima per compendium, quod os est, non ov $26 \alpha i \tau \omega]$

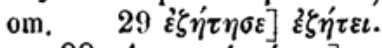

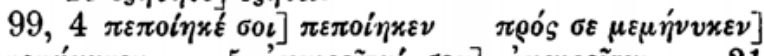

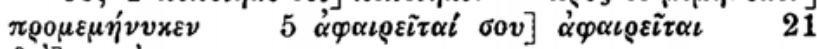
$\delta \iota \dot{\alpha}] \pi \alpha \rho \dot{\alpha}$. 0

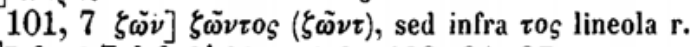
$24 ' I \varepsilon \beta 0 \sigma \vartheta \xi]$ i $i \varepsilon \beta 0 \vartheta \dot{~ h i c ~ e t ~ i n f r a ~ 102, ~ 24, ~} 27$.

$\left.\left.102,12 \pi \alpha \varrho o^{\prime} v \nu \varepsilon\right] \pi \alpha \rho \omega^{\prime} v \nu \varepsilon \quad 17 \delta \xi\right] \delta^{\prime}$ ut Wolfius $\left.28 \omega^{\tau}\right] \tau \omega ั$ perspicue.

$103,11 \alpha v \tau \dot{\prime} \nu]$ oin. $18 \pi \rho \tilde{\alpha} \xi \alpha \iota] \pi \rho \alpha^{\prime} \xi \varepsilon v$, sed $\alpha \iota$ margo r. $21 \varepsilon i \xi]$ om. $\quad 30$ roi $\alpha \lambda \lambda \omega s]$ s. v. ead. m.

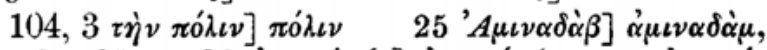

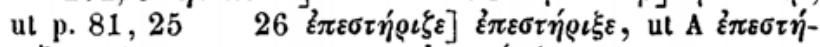
$\varrho \iota \xi \varepsilon$, unde ego iam scripseram $\varepsilon \pi \varepsilon \sigma \tau \eta \dot{\rho} \iota \xi \varepsilon$.

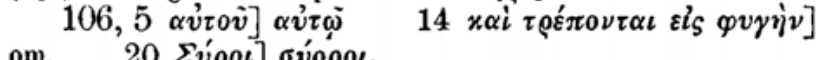

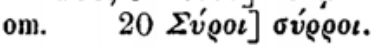

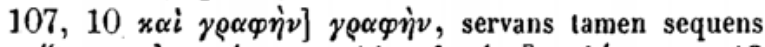

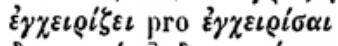
$\delta v \sigma \mu \alpha \chi \omega ́ \tau$.] $\delta v \sigma \mu \alpha \chi o ́ \tau$. $\beta \alpha \sigma \iota \lambda \varepsilon \dot{v}$.

$108,6 \delta \dot{\varepsilon}] \delta$ ' ut Wolfius et ego $\left.\tau \dot{\varepsilon} \vartheta \nu \eta x \varepsilon \delta \dot{\delta}^{2} \nu\right]$

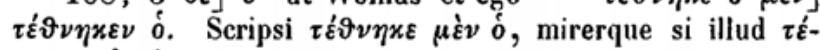
$\vartheta \nu \eta x \varepsilon$ o $\mu \dot{\varepsilon} \nu$ servet $\mathrm{A}$, de quo tacetur.

$\left.109,9 \varkappa \alpha \tau \alpha \sigma \pi \alpha \sigma \alpha \mu \varepsilon^{\prime} \nu \eta\right]$ prius $\sigma$ deletum r., ut $x \alpha \tau \alpha-$

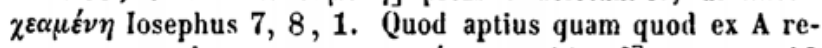
ceptum pro altero $x \alpha \tau \alpha \pi \alpha \sigma \sigma o \mu \varepsilon \dot{v} \eta \quad 11 \tau \omega]$ om. 16 $\delta \dot{\varepsilon}$ anguste insertum $32 \alpha \nu \varepsilon \lambda \varepsilon \tilde{\imath} \nu] \mathrm{om}$.

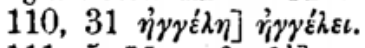

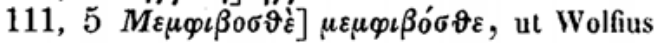




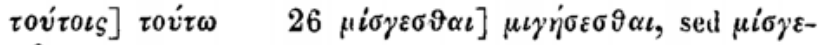
$\sigma \vartheta \alpha \iota$ margo $\mathrm{r}$.

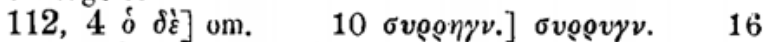

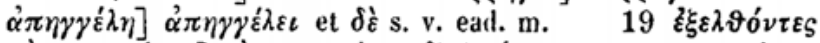

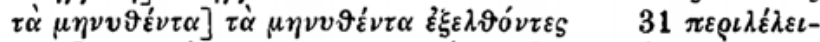
$\left.\pi \tau \alpha \iota] \pi \alpha \rho \alpha \lambda \varepsilon^{\prime} \lambda \varepsilon \iota \pi \tau \alpha \iota \quad \mu \alpha \chi \eta \sigma o ́ \mu \varepsilon \nu o \nu\right] \mu \alpha \chi \varepsilon \sigma \alpha \dot{\alpha} \mu \varepsilon \nu 0 \nu$.

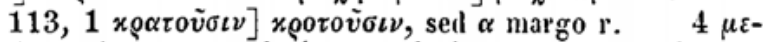

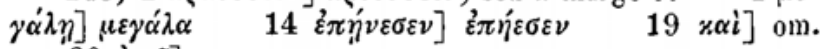

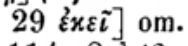

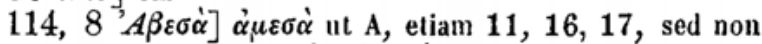
115, $\left.1629 \pi \alpha^{\prime} \lambda_{\iota v} \pi \alpha^{\prime} \sigma \eta \varsigma\right] \pi \alpha^{\alpha} \sigma \varsigma$, ut tacito ed. Bonn., haud dubie ex A. Sed quum etiam apud losephum 7, 11, 8

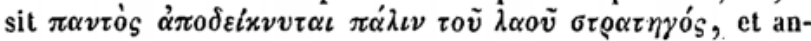
tea restitui quod casu excidisse videbatur in ed. Bonnensi, neque, si desit etiam in $\mathbf{A}$, delendum putem, alibi quoque omittente utroque libro quae sunt apud losephum, ut p. 106,

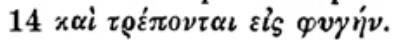

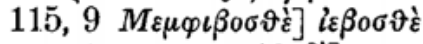

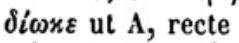

$19 \delta \dot{\varepsilon}]$ supra v. eadem $\mathrm{m}$.

$13 \kappa \alpha \tau \varepsilon \delta i \omega \xi \varepsilon]$ $\alpha \tau \varepsilon-$ va $\alpha$ ] $i \omega \nu \alpha \delta \alpha$, u videtur: nunc ente 26 ' $1 \omega$ $\left.\nu \alpha^{\prime} \vartheta \alpha \nu\right] i \omega \nu \alpha \delta \alpha^{\prime} \beta$, ut videtur: nunc enim erasis litteris mediis apparet tantum $i \omega \alpha \beta$, pro quo $i \omega \nu \alpha \vartheta \alpha^{\prime} \nu$ margo r.

$\left.30 \delta_{\xi}\right] \delta^{\prime}$ ut Wolfius et ego.

$116,9$ غ̇лi $B \eta \vartheta \lambda \varepsilon \dot{\mu} \mu]$ om.

$\tau \alpha \pi \lambda \alpha \gamma \varepsilon \dot{v} \nu \varepsilon \varsigma$, sed $\omega \nu$ margo r. $\left.15 x \alpha \tau \alpha \pi \lambda \alpha \gamma^{\prime} \nu \tau \omega \nu\right] x \alpha-$ $16 \pi \varrho 0 \sigma x \varepsilon x о \mu i x \alpha \sigma \iota \nu]$

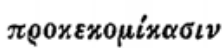
qui $\mu \varepsilon \mu \varepsilon \dot{\varepsilon} \lambda \eta \tau \alpha \iota$

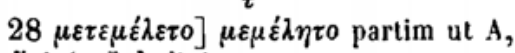
$\left.31 z^{\prime \prime} x \lambda \varepsilon \xi \alpha \iota\right] \varepsilon \pi i \lambda \varepsilon \xi \alpha \iota$.

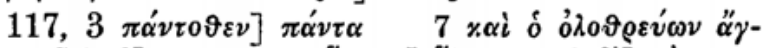

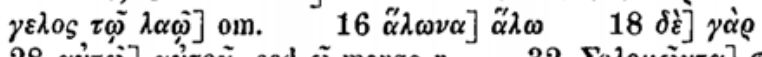

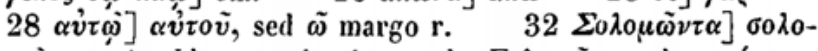

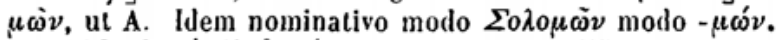

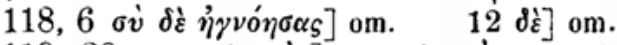

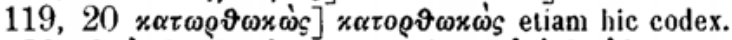

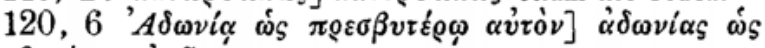
$\pi \rho \varepsilon \sigma \beta v \tau \varepsilon \dot{\varepsilon} \rho \alpha \dot{v} \tau \tilde{\omega}$.

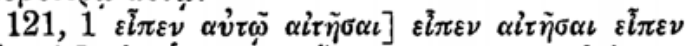

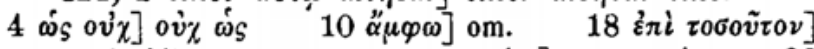

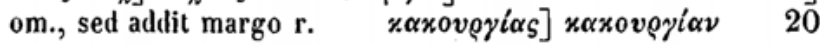




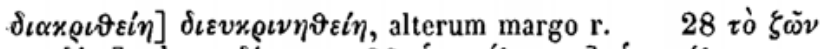

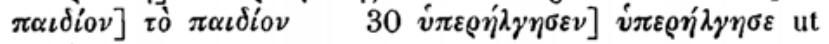
Wolfius et ego.

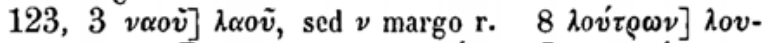

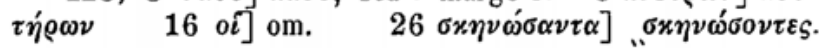

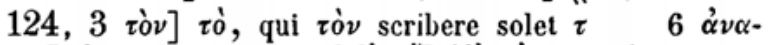
$\chi \vartheta \tilde{\eta} \nu \alpha \iota] \varepsilon^{2} \nu \varepsilon \chi \vartheta \dot{\eta} \nu \alpha \iota \quad 8 \varepsilon i \delta^{\prime}$ o'v] $i \delta^{\prime}$ ov', sed $\varepsilon i$ margo r.

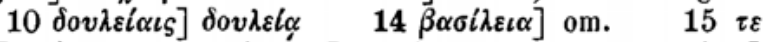

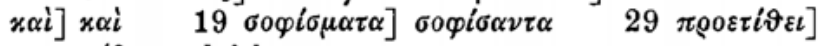
$\pi \varrho 0 \sigma \varepsilon \tau i \vartheta \varepsilon \iota$, sed deleto $\sigma \mathrm{r}$.

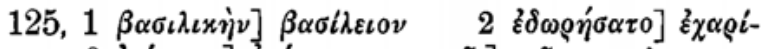

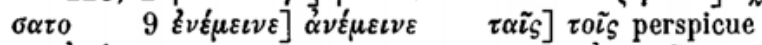

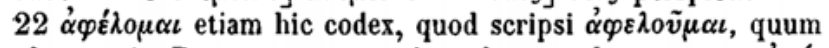
alterum in Zonaram non magis cadere crederem quam $\alpha \gamma \alpha \dot{\alpha}$.

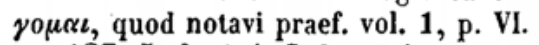

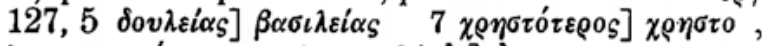

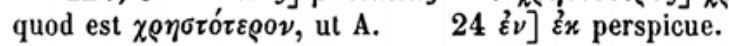

128, $7 x \alpha i$ om. Duc.] addit: sed unius Parisinae vitia pleraque omitto $9 x \alpha \tau \alpha \lambda \varepsilon i \psi \eta]$ ] $x \alpha \tau \alpha \lambda \varepsilon i \psi \varepsilon \iota$ ultima com-

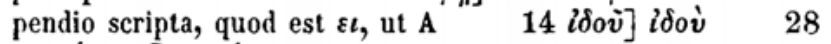
$\left.\chi v \vartheta \eta^{\prime} \sigma \varepsilon \tau \alpha \iota\right] \chi \varepsilon \vartheta \eta^{\prime} \sigma \varepsilon \tau \alpha \iota$.

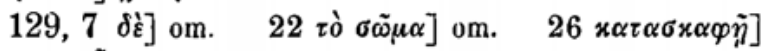
$\alpha \tau \alpha \sigma \kappa \alpha \varphi \tilde{\eta} \tau \varepsilon$.

$130,20 \mu \varepsilon \dot{v \varepsilon \iota]} \mu \varepsilon v \varepsilon \tilde{\imath}$ recte $25 \pi \alpha \rho \dot{\alpha}] \pi \varepsilon \rho i$, sed $\pi \alpha \varrho \dot{\alpha}$

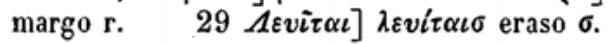

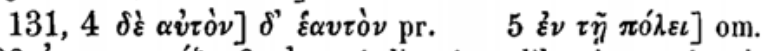
$\left.20 \stackrel{\alpha}{\alpha} \tau \iota \pi \alpha \rho \alpha \tau \alpha^{\prime} \xi \alpha \sigma \vartheta \alpha \iota\right]$ erasis litteris $\varrho \alpha$ librarius coniunxit

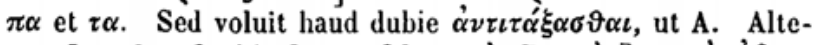
rum Iosephus $8,11,2 \quad 29 \pi \alpha \rho \dot{\alpha} B \alpha \alpha \sigma \dot{\alpha} \nu] \pi \alpha \rho \dot{\alpha} \alpha \beta \alpha \sigma-$ $\sigma \dot{\alpha} \nu$, ut $B \alpha \alpha \sigma \sigma \dot{\alpha} \nu$ hic et continuo Wolfiana, quae infra p. 132,

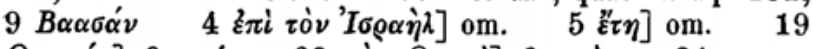
$\left.\left.\Theta \alpha \mu \nu \eta^{\prime} \nu\right] \vartheta \alpha \mu \nu i \quad 20 \tau \dot{0} \nu \Theta \alpha \mu \nu i\right] \vartheta \alpha \mu \nu i \quad 21 \pi \varepsilon \rho \varepsilon$ -

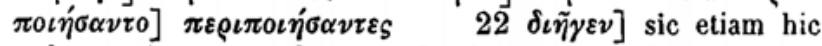
codex, qui $\varepsilon \nu$ non solum ascribit in rasura, sed etiam compendio usitato scribit super $\gamma \quad 23 \Sigma \alpha \mu \alpha \rho \alpha \iota \dot{\nu} \nu] \sigma o \mu \alpha \rho \alpha \iota \dot{\nu} \nu$

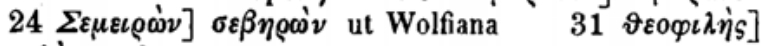
$\vartheta \varepsilon 0 \varphi v \lambda \dot{\eta} s$, sed $\iota$ margo r. 


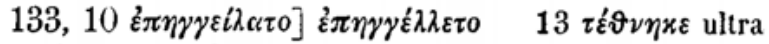
versum in marg. ead. $\mathrm{m}$.

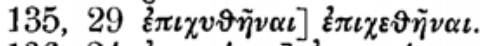

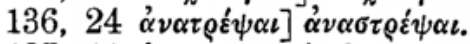

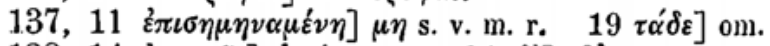

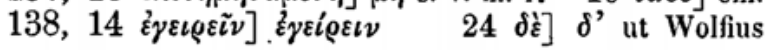
et ego.

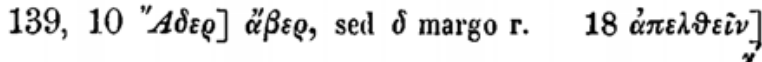
om. $23 \kappa \alpha \tau \alpha \delta \eta \sigma.] \kappa \alpha \tau \alpha \delta \varepsilon \sigma . \quad 26 \tau \alpha \xi i \alpha \varrho \chi 0 \nu] \tau \alpha \xi \ddot{i \alpha}{ }^{\chi}$, i. e. $\tau \alpha \xi \iota \alpha \varrho \chi \eta \nu$ ut A.

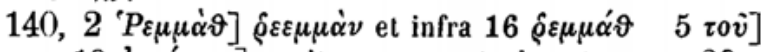

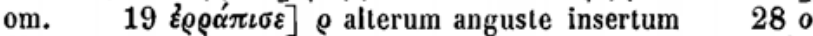
$\delta \frac{1}{\varepsilon}$ ex A additum etsi non habet, est tamen insertum ante $\tau \dot{\nu} \nu$ signum + , idemque in marg., sed nihil additum.

141, 9 ह̇ंi] $\pi \alpha \varrho \dot{\alpha}$ ut A, de quo dixi praef. vol. 1, p. V.

$\left.18 \delta^{\prime}\right]$ om. $\left.19 \pi \varrho \circ \sigma \chi \vartheta \hat{\sigma} \sigma \alpha \nu \tau \sigma \varsigma\right]$ sic etiam hic cod.

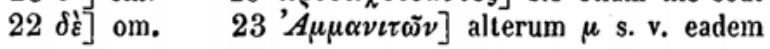
fortasse $\mathrm{m}$.

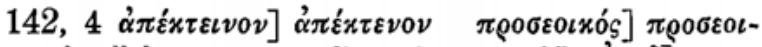

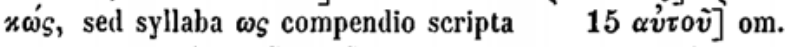

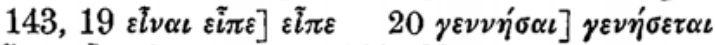

21 i $\left.\pi \pi 0 \iota_{\varsigma}\right] \tau o i_{S}$, sed ut p. 140,28 , signo ante hoc et in margine r., etsi nihil ascriptum 23 ö $]$ om.

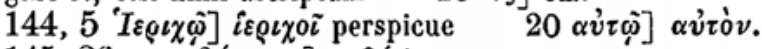
$145,26 \pi \varrho \varepsilon \sigma \beta v \tau \alpha \tau o \nu] \varepsilon \sigma \beta v^{\prime}$ in rasura.

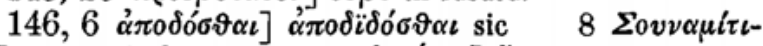

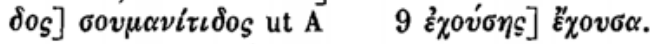

$147,4 \tau 0 \tilde{v}]$ o perspicue $6 x \alpha \tau \alpha \lambda \varepsilon i \psi 0 v \sigma \iota]$ super $\sigma \iota$ positum ${ }^{2}$ significat $\sigma \iota \nu$. Tum $\pi \varepsilon \rho \iota \sigma \varepsilon v \dot{v} \mu \alpha \tau \alpha$, ut $19 \dot{\alpha} \dot{\lambda} \alpha \sigma \sigma o \mu$. $11 \varepsilon i]$ om., sed supplet margo r.

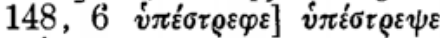

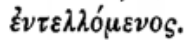

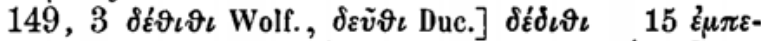

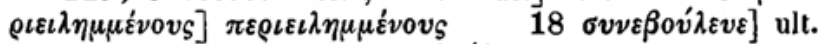
in rasura, ut nunc videatur $\sigma v \nu \varepsilon \beta 0 v i \lambda \varepsilon v \sigma \varepsilon$, ut $\mathrm{A} \quad 26 \pi \varepsilon v$ -

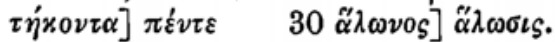


$150,21 \alpha \hat{v} \tau \tilde{\omega} \nu]$ om. quod A transponit post $\stackrel{\alpha}{\alpha} \tau \iota \tau \tau i \alpha \nu$, ut solet Zonaras verba transponere.

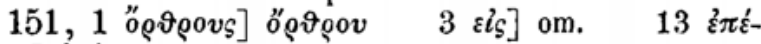

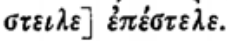

$\left.152,22 \tau \tilde{\omega} \nu] \tau \tilde{\omega} \tau \tilde{\omega} \nu \quad 25 \tau \tilde{\eta}_{S} \sigma \tau \rho \alpha \tau i \tilde{\alpha}_{S}\right]$ om., ut losephus A. I. 9,6, 1 , sed qui praemiserat $\tau \dot{\eta} \nu \sigma \tau \varrho \alpha \tau \iota \dot{\alpha} \nu$ «ँ $\pi \alpha \sigma \alpha \nu$.

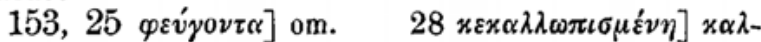
$\lambda \omega \pi \iota \sigma \mu \varepsilon \dot{\varepsilon} \nu \eta \quad 32 \tau \omega]$ om. ut A. Sed habet Iosephus 9, 6, 4 .

$\left.154,5 \delta_{\xi}\right] \delta$ ut Wolfius et ego $\left.17 \delta \dot{\varepsilon}\right]$ om. ut A,

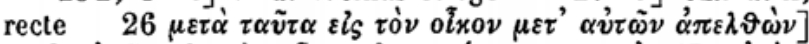
$\mu \varepsilon \tau^{\prime} \alpha \dot{v} \tau \tilde{\omega} \nu \varepsilon l_{\zeta} \tau \dot{\nu} \nu$ oixov $\left.\alpha \pi \varepsilon \lambda \vartheta \omega^{\prime} \nu \quad 32 \alpha \dot{v} \tau 0 \tilde{v}\right] \alpha \dot{v} \tau \dot{\eta} v$, ut A. Et ipsam Wolfius, ut $\alpha$ v่vov fortasse fide careat.

$155,3 \beta \alpha \sigma \iota \lambda \varepsilon i \alpha \nu \alpha v i v 0 \hat{]}] \beta \alpha \sigma \iota \lambda \varepsilon i \alpha \nu \quad 14 \dot{\eta}]$ o 25 $\lambda \alpha \grave{o ̀ s}_{\text {] }} \beta \alpha \sigma \iota \lambda \varepsilon \dot{v}$.

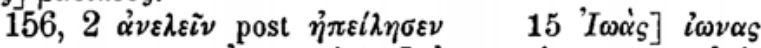

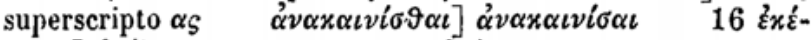
$\lambda \varepsilon v \sigma \varepsilon] \varepsilon \dot{x} \varepsilon \dot{\lambda} \varepsilon v \varepsilon$, ut Wolfius. Sed $\varepsilon^{x} \varepsilon \dot{\lambda} \varepsilon v \sigma \varepsilon$, ut Duc., ad quod

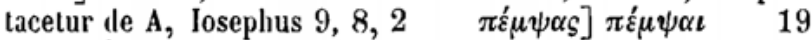
$\lambda 0 \gamma \iota \sigma \vartheta \eta \dot{\sigma \varepsilon} \sigma \vartheta \alpha \iota] \lambda 0 \gamma \iota \sigma \vartheta \varepsilon i_{S}$ et $\sigma \vartheta \tilde{\eta} \nu \alpha \iota$ margo r. $23 \dot{\varepsilon} \mu-$

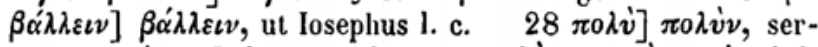

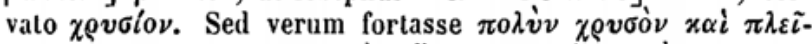

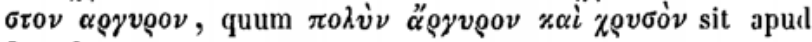
Iosephum.

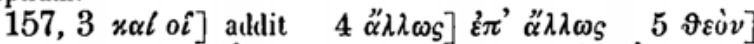
s. v. ead. m. $\left.\quad 6 \delta^{\prime}\right] \delta \dot{\varepsilon}$ ut A, recte $\left.10 \delta \dot{\varepsilon}\right] \delta$ ' ut Wol-

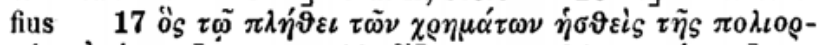

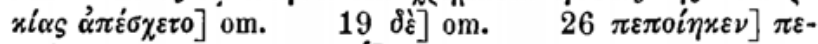
$\pi$ oinxe ut Wolfius 27 o] om.

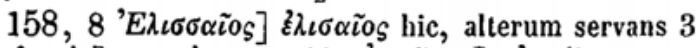
15 $\operatorname{mox} \tau 0 \tilde{v} 31$ ôs $x \alpha i]$ ôs $32 \tau \omega \nu]$ om. ut A, recte.

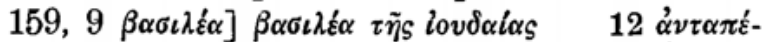
$\sigma \tau \varepsilon \iota \lambda \varepsilon]$ etiam hic codex, quod alibi ipse mutat in $\alpha \nu \tau \varepsilon \pi \varepsilon-$ $\sigma \tau \varepsilon \iota \lambda \varepsilon$, quod hic restituendum. V. ad vol. 3, p. 310, 29 $\left.16 \varepsilon \dot{v} v \dot{v} \chi \eta \alpha_{S}\right] \cdot \varepsilon \dot{v} \tau \chi \eta^{\prime} \sigma \alpha_{S} \quad 29$ हैं $\eta$ om. ¿ैं $\left.x \alpha i \delta \varepsilon^{\prime} x \alpha\right]$ $\varepsilon \xi x \alpha i \delta \varepsilon x \alpha$. 
$160,5 \tau \alpha \pi \varepsilon \iota \nu \omega \vartheta \varepsilon \dot{\varepsilon} \nu \tau]$ ] $\tau \alpha \pi \varepsilon \iota \nu \omega \sigma \alpha \nu \tau \alpha \quad 15 \varepsilon \alpha v \tau \dot{\varepsilon} \nu]$ $\alpha v \tau o ́ v$, qua forma non utitur Zonaras, quod tenendum propter locos quosdam quibus alterum necessarium videatur.

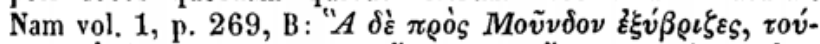

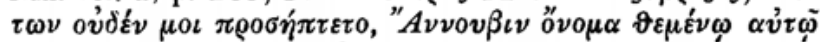
quod Pinderus scripsit $\alpha \dot{v} \tau \tilde{\omega}$ neque in Parisino A, de quo tacetur, esse credo neque a Zonara scriptum, etsi ne $\alpha v \tau \tilde{\omega}$ quidem locum habet. Nam haud dubie $\varepsilon \mu \alpha v \tau \tilde{\omega}$ ob praecedens $\omega$ $\vartheta \varepsilon \mu$, sic cum ductu post $\mu$ scriptum in Monacensi, in $\alpha v \tau \tilde{\omega}$ est mutatum $\tau \dot{\alpha}] \tau \dot{\eta} \nu$, scriptum $\stackrel{\Lambda}{ }^{\prime}, \quad 19 \delta$. $\dot{v} \pi \dot{\delta} \Sigma$. $\left.\dot{\alpha} \sigma \varepsilon \beta \tilde{\omega}_{S} \beta \iota 0 v_{\varsigma}\right] \stackrel{\alpha}{\alpha} \sigma \varepsilon \tilde{\omega}_{S} \beta \iota \tilde{v}_{S}$ ante $\delta$. vंrò $\sigma$.

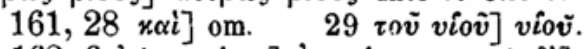

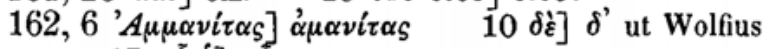
et ego 17 ov ó ov́.

$163,3 \tau \dot{0} \nu] \tau \tilde{\omega} \nu \quad 9 \Theta \alpha \iota \gamma \lambda \alpha \varphi \alpha \lambda \alpha \sigma \dot{\alpha} \varrho] \vartheta \alpha \iota \gamma \lambda \alpha \varphi \alpha \lambda \alpha$ -

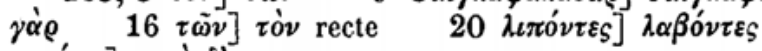
$\mu \varepsilon \tau \dot{\alpha} \tau \varepsilon] \mu \varepsilon \tau \dot{\alpha} \delta \dot{\varepsilon}$.

$165,1 \delta \dot{\varepsilon} \pi \alpha \rho \dot{\alpha} \mu \dot{\varepsilon} \nu] \mu \dot{\varepsilon} v \pi \alpha \rho \dot{\alpha} \quad \tau \rho \iota \alpha x o ́ \sigma \iota \alpha] \tau \rho \iota \alpha x \tilde{o}^{\prime}$

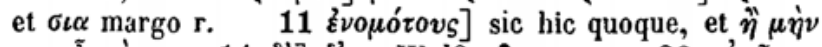
pro $\left.\tilde{\eta}^{\tilde{\eta}} \mu \dot{\eta} v \quad 14 \delta \dot{\varepsilon}\right] \delta^{\prime}$ ut Wolfius et ego $22 \alpha v \tau \tilde{\eta} \tau \varepsilon-$ $\vartheta \lambda \alpha \sigma \mu \varepsilon \dot{v} \eta] \alpha \dot{v} \tau \dot{\eta} \tau \varepsilon \vartheta \lambda \alpha \sigma \mu \varepsilon \dot{v} \eta$.

$166, \overline{6} \tau \tilde{\omega}] \tau \tilde{\omega} \tau \tilde{\omega}$.

167, 29 'B $\alpha \beta v \lambda \omega v i \omega] \beta \alpha \sigma \iota \lambda \varepsilon i \beta \alpha \beta v \lambda \omega v i \omega$.

$\left.168,2 \mu \eta^{\prime}\right]$ in rasura $3 \varphi \eta \sigma i$ (pro $\varphi \eta \sigma i v$, quod scripsi)] $\varphi \dot{\eta} \quad 10 \delta \dot{\varepsilon}]$ om. $\quad 17 \delta \dot{\varepsilon}] \delta^{\prime}$.

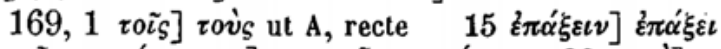

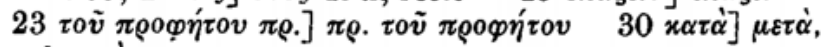
sed $x \alpha \tau \dot{\alpha}$ margo r.

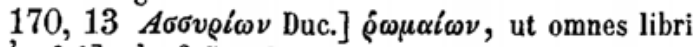
$27 \alpha \sigma \varepsilon \beta \varepsilon i] \varepsilon v \dot{v} \sigma \varepsilon \beta \varepsilon \tilde{\imath}$, sed $\alpha$ s. v. r.

$171,8 \dot{\alpha} \lambda \lambda \dot{\alpha} x \alpha i] \dot{\alpha} \lambda \lambda \dot{\alpha}$.

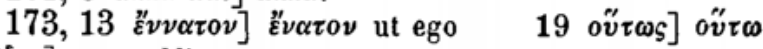
$\pi \alpha i \delta \alpha s]$ om., addit margo $\mathrm{r}$.

$174,14 \dot{\alpha} \lambda \eta \vartheta \tilde{\omega} s] \dot{\alpha} \lambda \eta \vartheta$, littera $\vartheta$ cum ductu, qui $\omega s$ videtur $20 \delta$ ] post hoc asteriscus r., sed nihil in marg.

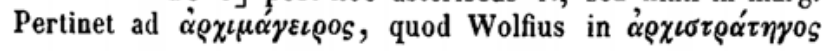


mutatum volebat $\quad 30 \mu \varepsilon \dot{\varepsilon} \varrho \iota \varsigma] \mu \dot{\varepsilon}$, altera parte superscripta cum compendio quod $\chi \varrho \iota$ videtur.

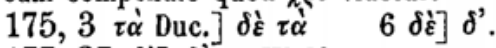

$177,27 \delta \dot{\varepsilon}] \delta$ 'ut Wolfius.

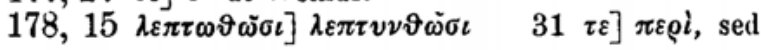
$\tau \varepsilon$ margo.

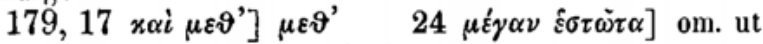
A, quae habet Iosephus 10, 10, 4.

$180,18 \alpha v^{3} \iota \varsigma$ ] om. ut A, recte $\left.23 \dot{o}\right] \omega_{\varsigma}$, sed $\delta$ margo r. $\left.27 x \alpha \tau \varepsilon^{\prime} \lambda v \sigma \varepsilon\right] x \alpha \tau \varepsilon^{\prime} \lambda v \sigma \varepsilon v$, ultima compendio expressa $32 \dot{v} \pi 0 \tau v \pi 0 v i \alpha \iota] v$ in rasura, pr. fortasse $\alpha$.

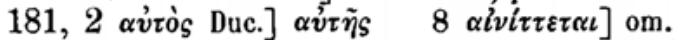

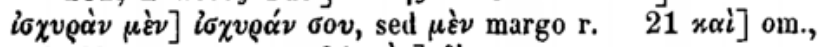

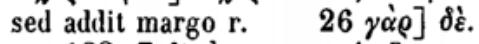

$182,7 \xi \xi \xi \alpha \rho \omega \tau 0 x \rho \alpha \tau i \alpha \varsigma]$ om. ut A, recte $8 \varepsilon i x \alpha i]$ x $\alpha i$ $\left.11 \tau \tilde{\omega} \nu] \tau \dot{\eta} \nu \quad 26 \dot{\alpha} \sigma \vartheta \varepsilon v 0 \tilde{v} \sigma \alpha \nu] \dot{\alpha} \mu \varepsilon \lambda_{0} \tilde{v} \sigma \alpha \nu \quad 27 x \alpha i\right] \dot{\eta}$.

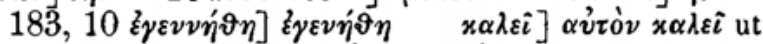
$\mathrm{A}$, quod recipi potest deleto $\tau$ ò $\mathrm{X} \iota \iota \tau \tau o ́ v$, quod om. hic codex, non A.

$[184,12$ corr. dó $\mu \alpha \tau \alpha$.

$185,12 " I \varrho]$ ante hoc asteriscus r., sed nihil in marg. $28 \varphi \eta \sigma i] \varphi \eta$ cum ductu infra $\eta$. Scripsi $\varphi \eta \sigma i \nu \quad 32 \mu \varepsilon-$ $\gamma \alpha \lambda o \sigma v v \eta] \mu \varepsilon \gamma \alpha \lambda \omega \sigma v^{\prime} \nu \eta$, ut ego, hic et continuo.

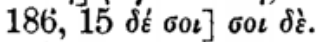

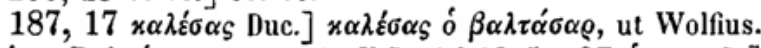
$x \alpha \lambda \varepsilon_{\sigma} \alpha \varsigma B \alpha \lambda \alpha^{\prime} \sigma \alpha \rho$, ut ego, A. V. Daniel 12,5 27 o $\left.x \rho \alpha \tau \tilde{\omega} \nu\right]$

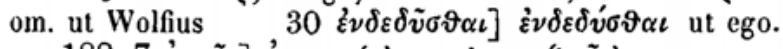

$\left.188,7 \dot{\alpha} \propto \chi \tilde{\eta}_{\varsigma}\right] \stackrel{\alpha}{\alpha} \omega \omega \eta \varsigma$ (ob proximum $\zeta \omega \tilde{\eta} \varsigma$ ), supra versum fere scriptis litteris $\dot{\alpha} \rho$ et $\eta_{S}$ et lineola infra verba $x \alpha i-\sigma o v$ ducta r. $\quad 8 \pi \varepsilon \varrho \iota \varepsilon ́ \sigma \tau \eta] \pi \varepsilon \rho i \varepsilon \sigma \tau \hat{\imath}$ ita cum ductu super $\iota$, circumflexo simili, qui significat $\nu \quad 9 x \alpha i] \gamma \dot{\alpha} \varrho \quad 14 \delta \dot{\varepsilon}]$ addit.

$189,10 \xi \xi \mu \beta \alpha \lambda \lambda \varepsilon \sigma \vartheta \alpha \iota] \quad z \mu$ in codice, ut saepe, velut p. 190,3 , ita scriptum ut $\varepsilon^{\prime} x$ legi possit, fecit ut $\varepsilon \mu$ pone-

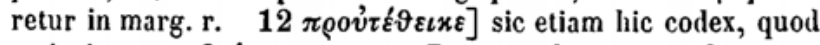
scripsi $\pi \varrho 0 v \tau \varepsilon \vartheta \varepsilon i x \varepsilon \iota$, ut saepe Zonaras plusquamperfecta ponit pro aliis temporibus 13 oí $] \varepsilon \dot{l}$, sed of margo r. 20 $\ddot{\eta} \lambda \pi \iota \sigma \varepsilon] \ddot{\eta} \lambda \pi \iota \xi \varepsilon$. 
190,4 $4 i \pi \omega \nu]$ om. $\quad 8 \delta i \grave{x} x \alpha i] \delta \imath$.

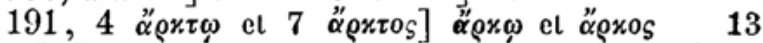
$\left.\delta \eta^{\prime} \lambda \eta v\right] \delta \tilde{\eta} \lambda \alpha$, circumflexo in acutum mutato $\left.14 i^{\prime} \sigma \alpha_{S}\right]$

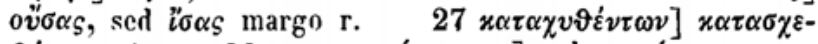

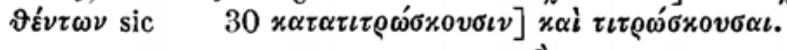

$192,3 \alpha \dot{v} \tau \dot{\alpha} \varsigma]$ ] $v_{\tau o ̀ s}$, scriplum $\alpha \dot{v} \tau$, quod mili quoque

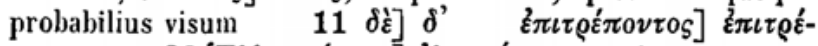

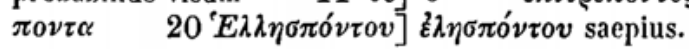

$\left.193,25 \mu \varepsilon^{\prime} \chi \rho \iota\right] \mu \alpha \varrho$ et super his $\mathrm{j}$, sic fere, et $\mu \dot{\varepsilon}^{\prime}-$ $\boldsymbol{i}{ }^{i} /$

$\chi \varrho \iota$ margo r. Solet enim $\mu \varepsilon$ in hoc codice scribi pro $\mu \varepsilon \chi^{\prime} \varrho \iota \varsigma$,

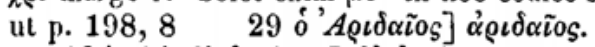

$\left.194,14 \delta \delta_{\varepsilon}^{\prime} \varepsilon \varepsilon^{\prime} i v \omega \nu\right] \delta^{\prime} \varepsilon^{\prime} x \varepsilon i v \omega v$ ut Wolfius et ego.

$195,8 \delta \alpha \sigma \mu \tilde{\omega} \nu] \alpha$ in ras., pr. fortasse $\varepsilon \quad 11 \delta \xi] \delta^{\prime}$

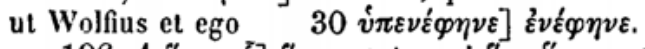

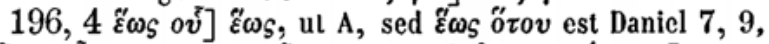

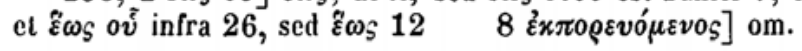

197, 16 oै $\psi \varepsilon \sigma \vartheta \alpha \iota]$ oै $\psi \varepsilon \sigma \vartheta \alpha \iota$ etiam cod., sed ultima com-

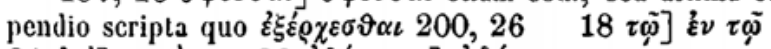

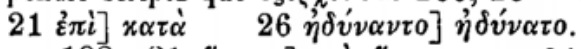

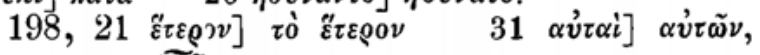
sed scriptum $\alpha \widetilde{v \tau}$.

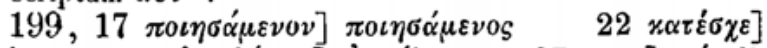

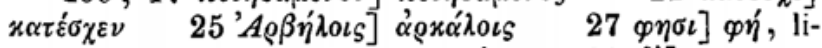

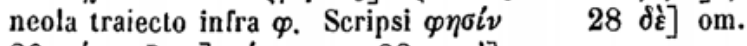

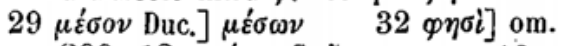

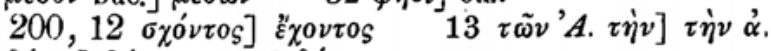
$\left.30 \delta v^{\prime} \sigma \nu\right] \delta v^{\prime} \nu \alpha \mu \iota \nu$, sed $\delta v^{\prime} \iota \nu$ margo r.

202, $2 \varphi \eta \sigma i]$ eolem fere compendio quo ante. Scrib.

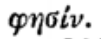

$203,6 \tau \tilde{\omega} \Delta \alpha \nu \iota \dot{\eta} \lambda] \delta \alpha \nu \iota \dot{\eta}^{\prime} \lambda$.

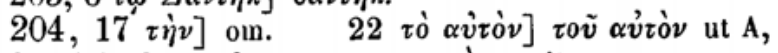
quod recipiendum colo post $\sigma \eta \mu \alpha \nu \tau \iota x o ̀ \nu$ posito.

205, 1 q $\left.\eta \sigma_{i \nu}\right]$ om. $\left.\quad 7 \delta \dot{\varepsilon}\right]$ addit.

$206,5 \sigma \omega \dot{\xi} \varnothing \nu] \sigma \widehat{\omega} \xi \mathrm{ut}$ solet. Seclusi $x \alpha \tau \alpha \dot{\alpha} \quad 7 \beta \alpha \sigma \iota-$ 


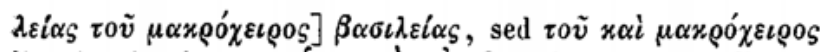

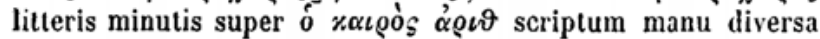
ab ea quae marginibus ascripta allevit et similior est ei quae codicem scripsit $\left.\left.9 \delta \delta^{\prime} \circ \nu\right]\right]_{\tau \varrho^{\prime}} \delta \varepsilon^{\prime}$, sed $\delta \dot{\varepsilon} o \nu$ margo r. $\delta \varepsilon{ }^{\nu}$ scriptum $24 \dot{\alpha} \nu \omega \dot{\tau \varepsilon \rho o \nu] ~} \dot{\alpha} \nu \omega$, quod item est $\dot{\alpha} \nu \omega ́ \tau \varepsilon \rho \circ \nu$, non $\dot{\alpha} \nu \omega \tau \varepsilon \dot{\rho} \rho \omega$.

$\omega$

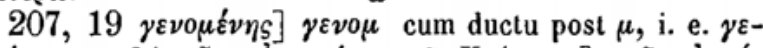

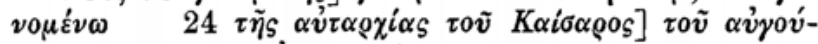
$\sigma \tau o v$ (hoc scriptum $\alpha v$, ceteris compendio superscriptis litte-

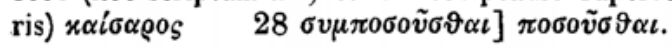

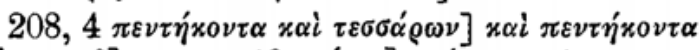

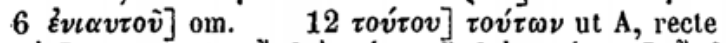

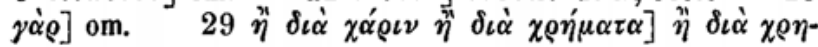

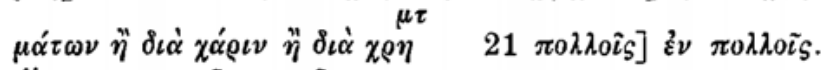

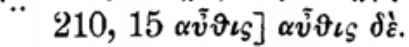

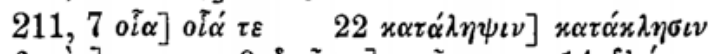

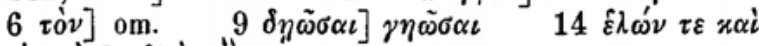
$\dot{\alpha} \nu \varepsilon \lambda \dot{\omega} \nu \tau \dot{o} \nu]_{\text {, }} \hat{\varepsilon} \lambda \dot{\omega} \nu \stackrel{\tau}{\tau}$, sed super $\omega$ accentu acuto in gravem mutato et $\tau$ ita scripto ut alter gravis in acutum mutatus videatur manu sec.

212, 30 ö $\tau \omega]$ ö $\pi \eta$, ut A ö $\pi \rho$.

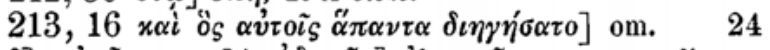

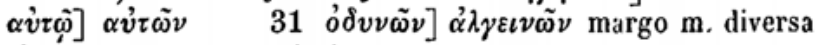
ab ea quae cetera ascripsit.

$214,3 \stackrel{\eta}{\eta}] \ddot{\eta}$ ut Wolfius $\left.\left.15 \not{\alpha} \beta \rho \alpha_{S}\right] \stackrel{\alpha}{\alpha} v \alpha_{S} \quad \alpha \dot{v} \tau \tilde{\eta}_{S}\right]$ $\eta$ ambigue an $0 \iota$.

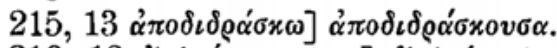

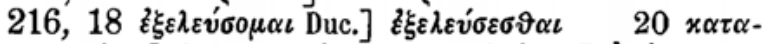

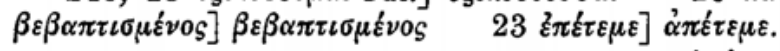

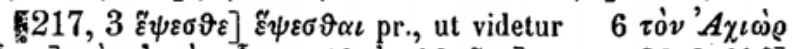

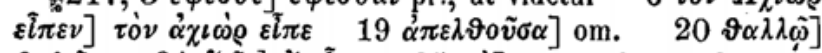
$\left.\left.\vartheta \alpha \lambda \hat{\omega} \quad 24 \hat{\varepsilon} \xi \tilde{\eta}_{s}\right] \xi \xi \tilde{\eta} \tilde{\eta}_{S} \quad 25 \tau \dot{\alpha}\right]$ s. v. ead. ut videtur m. $29 \alpha \nu \vartheta \rho \omega ́ \pi 0 v \varsigma] \alpha \tilde{\alpha o v}$, sed $\alpha \nu$ in ras.

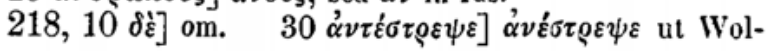


fius et ego, neque aliter, ut opinor, A, quamvis taceat collatio Haasii.

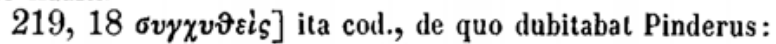
v. praef. vol. 1, p. VI $\left.20 \varepsilon^{2} \nu\right]$ s. v. ead. ut videtur manu

$23 \mu \dot{\varepsilon} \nu]$ om.

220,5 عi] $\varepsilon i_{S}$ pr., ut videtur, quum erasa sit littera post $\varepsilon i$.

$221,26 \dot{o} \sigma \mu \dot{\eta} \tau o \tilde{v}] \dot{o} \omega \dot{\eta}$.

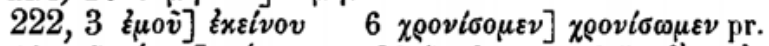
$10 \tau 0 \tilde{v} \gamma \alpha \dot{\alpha} \mu v] \gamma \alpha \dot{\alpha} \mu v \quad 20$ (ita leg. pro 25) vióv $\alpha \dot{v}$ $\left.\left.\tau \tilde{\eta}_{S}\right] v \hat{i} \hat{\nu} \nu 23 \mu \dot{\varepsilon} \lambda o \nu\right] \mu \dot{\varepsilon} \lambda \lambda o v$.

$223,2 T \omega \beta i \tau] \tau \tilde{\omega}$ ante hoc cum lineola infra $\mathbf{r}$. है $\left.v \delta \circ \xi_{0} \nu\right]$ है $\left.v \delta \delta^{\prime} \xi \omega s \quad 23 \delta \dot{\varepsilon}\right], 0 \mathrm{~m}$.

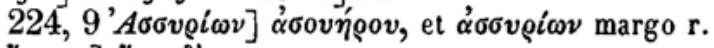

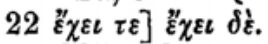

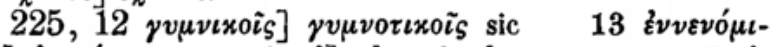

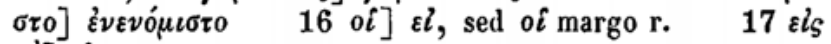
$\tau \dot{\alpha}] \varepsilon l_{s}$.

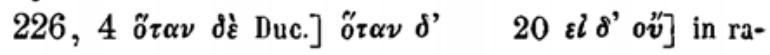

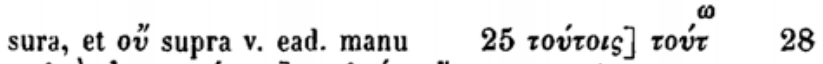

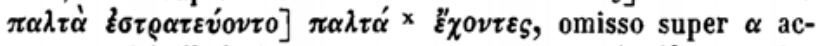
centu, nisi illud signum acutum et gravem significat potius quam vitium.

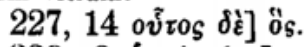

$228,3 \dot{\eta} \tau o i \mu \alpha \zeta \varepsilon v]$ pro $\dot{r}_{1}$ pr., ut videtur, $\left.v \quad 22 \varepsilon v\right]$

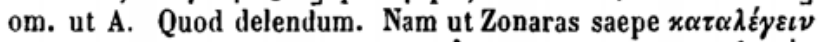

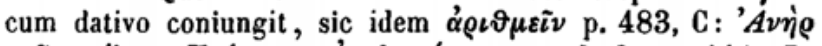

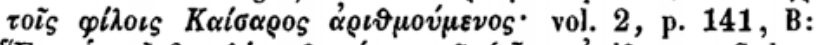

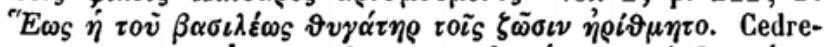

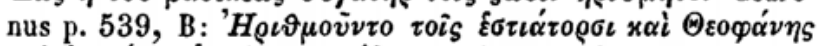

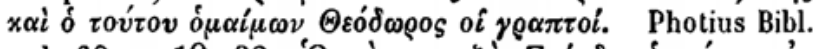

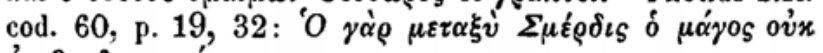

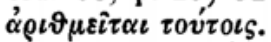

229,8 x $\alpha i \dot{\alpha} \mu \varphi$.] $\alpha \mu \varphi$.

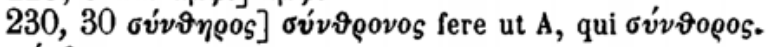

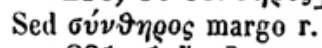

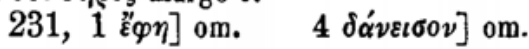


232, $2 \pi \varepsilon \varrho \iota \varrho \eta \gamma \nu.] \pi \alpha \varrho \alpha \varrho \varrho \eta \gamma \nu . \quad \delta \varrho v \pi r o ́ \mu \varepsilon \nu \alpha \iota] \vartheta \varrho v-$

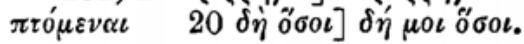

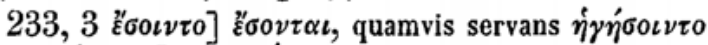
$\left.5 x \alpha \tau \alpha \lambda \eta^{\prime} \psi \circ \iota \tau 0\right]$ x $\alpha \tau \alpha \lambda \eta^{\prime} \psi \circ \iota \nu \tau 0$, sed erasum $\nu 11 M \eta^{\prime}-$ $\delta \omega \nu]$ supplet margo r. $13 \dot{\xi} \xi \tilde{\eta} \gamma \varepsilon] \bar{\varepsilon} \xi \tilde{\eta} \lambda \vartheta \varepsilon$, sed $\dot{\varepsilon} \xi \tilde{\eta} \gamma \varepsilon$

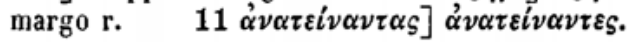

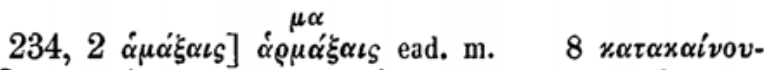
$\sigma \iota \nu] x \alpha \tau \alpha x \alpha i o v \sigma \iota v$, sed $x \alpha \tau \alpha x \alpha i v 0 v \sigma \iota v$ margo r. $23 \pi \rho 0-$

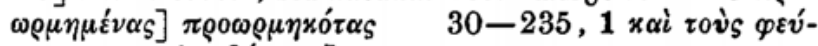

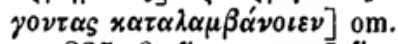

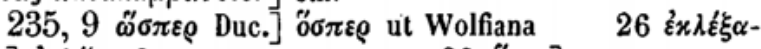

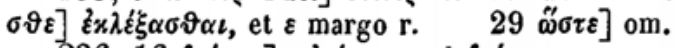

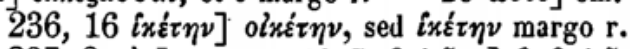

237, $2 \mu \dot{\varepsilon} \nu]$ om. $12 B \alpha \beta v \lambda \omega \nu \alpha] \beta \alpha \beta v \lambda \tilde{\omega} \nu S_{0} \quad 20$

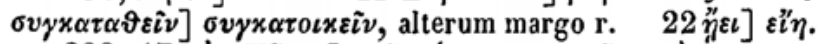
238, $17 \tau \dot{v} v K \tilde{v} \varrho o v]$ ] $0 \tilde{v}$ xv́ov, sed $\tau o \tilde{v}$ in $\tau \dot{o} v$ mutato,

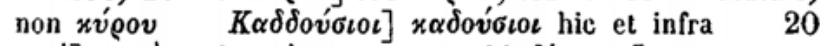

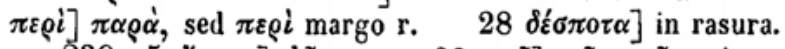

$239,5 i \omega \mu \varepsilon \nu] i \tilde{\omega} \mu \varepsilon \nu \quad 20 \tau 0 \tilde{v}] \tau \tilde{\omega} \nu \tau o \tilde{v}$ satis commode. ทें $2 \dot{\alpha}$.

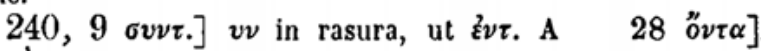

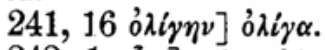

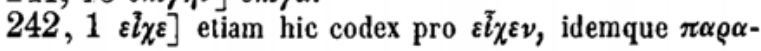

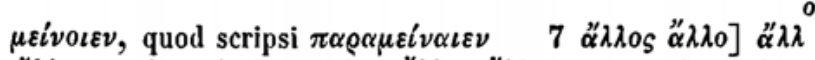
$\not \lambda \lambda_{0}$, quod est in hoc codice $\alpha^{\prime} \lambda \lambda_{0 \varsigma} \not \alpha \lambda \lambda_{0}$, non quod A habere dicitur " $\left.\alpha \lambda \lambda_{0} \alpha^{\prime \prime} \lambda \lambda_{0} \quad 15 \lambda \alpha \nu \vartheta \alpha^{\prime} \nu \varepsilon \iota\right] \lambda \alpha \nu \vartheta \alpha^{\prime} \nu \varepsilon \sigma \vartheta \alpha \iota$, sed $\varepsilon \iota$ margo r. $18 \delta v \nu \eta \sigma o ́ \mu \varepsilon \vartheta \alpha] \delta v \nu \alpha ́ \mu \varepsilon \vartheta \alpha$.

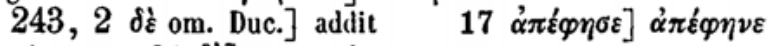
perspicue $31 \delta \dot{\varepsilon}]$ s. v. ead. m.

$245,11 \pi 0 \lambda \varepsilon \mu l o v s] \pi 0 \lambda \varepsilon ́ \mu 0 v s \quad 16 \gamma \dot{\alpha} \varrho]$ om. recte

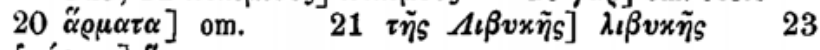
$\varepsilon x \alpha \dot{\sigma} \sigma \eta \nu]$ है $\alpha \alpha \sigma \tau \alpha$.

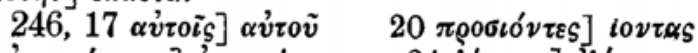

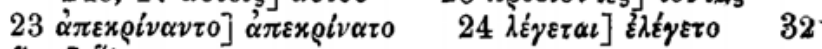
$\ddot{\eta} x \varepsilon \iota] \ddot{\eta} \xi \varepsilon$. 


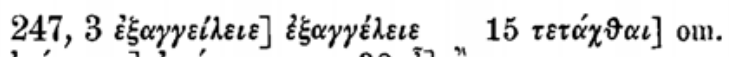

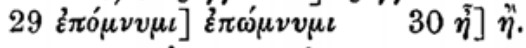

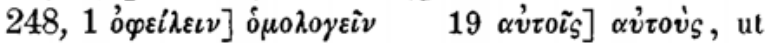
A. Sic p. 112, B, accus. pro dat. iidem praebent post $\pi \alpha \rho \alpha t-$

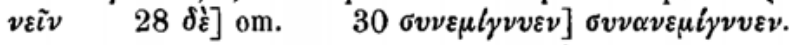

$249,2 \tau \tilde{\omega} \nu]$ om. $\quad \varepsilon \pi i] \varepsilon v \quad 3 \delta K \tilde{v} \rho \circ \varsigma] x \tilde{v} \rho \circ \varsigma$

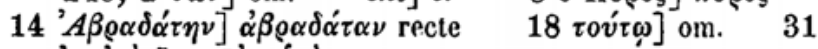
$\left.x \alpha \tau^{\prime} \alpha \dot{v} \tau \dot{0} v\right] x \alpha v^{\prime} \alpha \dot{v} \tau \dot{\nu} \nu$.

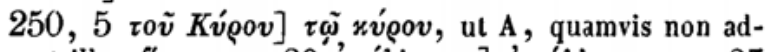

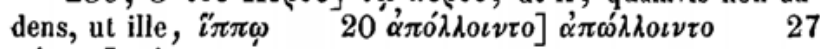
$\pi i \sigma \tau \varepsilon \iota \varsigma] \pi i \sigma \tau \iota v$.

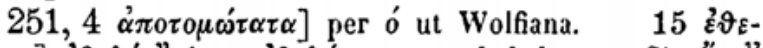

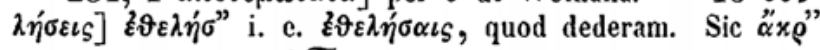

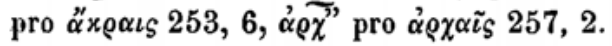

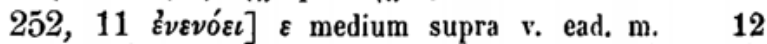

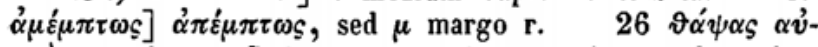
$\tau o v s \mu \varepsilon \gamma \alpha \lambda o \pi \rho \varepsilon \pi \tilde{\omega}$ in summo paginae margine supplet eadem manus.

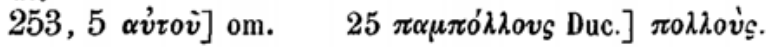

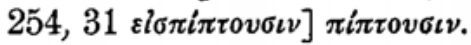

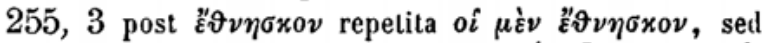
linea infra ducta notata r. $\left.27 x \alpha \tau \alpha \sigma x \varepsilon v \alpha^{\prime} \sigma \alpha l\right] x \alpha \tau \alpha \sigma x \varepsilon v \alpha^{\prime}-$ $\sigma \alpha \sigma \vartheta \alpha t$.

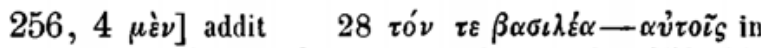
imo paginae margine suppleta $\mathrm{m}$. rec., de qua ad p. 259, 11, omisso, ut in A, quod habet Xenophon $\dot{\omega} \sigma \alpha \dot{\tau} \tau \mathrm{s}$.

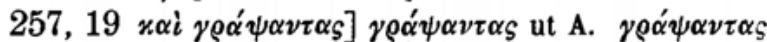

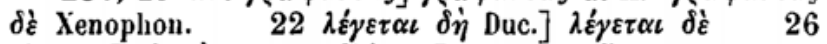
$\varepsilon i_{S}$. Duc.] $\left.\varepsilon i_{S} \tau \dot{\alpha} \quad 31 \varepsilon \xi \xi \xi \varphi \varepsilon \rho\right]$ ante hoc $\varepsilon^{\prime \prime} \varphi \eta$, sed lineola infra ducta r. $\left.\quad 32 \pi \alpha \mu \pi{ }^{\prime} \lambda \alpha_{\varsigma}\right] \pi \alpha \mu \pi o^{\prime} \lambda \lambda_{0 \varsigma}$, sed $\alpha$ super

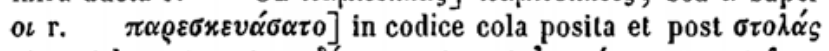

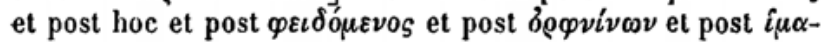
$\tau i \omega v$. Quod notavi ob interpunctionem olim apud Xenophontem male positam sic ut $\pi \alpha \mu \pi o ́ \lambda \alpha_{S}-i \mu \alpha \tau i \omega \nu$ parenthesi includerentur.

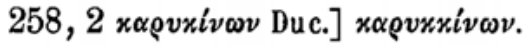




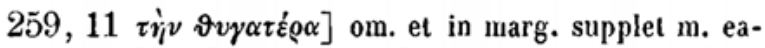
dem quae p. 256,30 versum in fine paginae, diversa ab ea

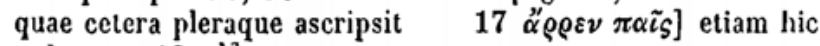
codex $18 \tau \dot{\alpha}]$ om.

$260,21 \alpha \dot{v} \tau 0 \tilde{v}] \alpha \dot{v} \tau \omega$

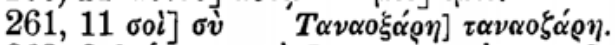

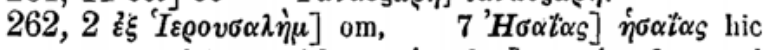
el mox, ut antea fuit $16 \sigma v v \alpha i \rho \varepsilon \sigma \vartheta \alpha \iota] \sigma v \nu \varepsilon i \varrho \varepsilon \sigma \vartheta \alpha \iota$, sed $\alpha \iota$ margo $r$.

$263,1 \delta \dot{\varepsilon}]$ om. $\quad 4 \varepsilon i] \varepsilon i_{\varsigma}$, sed deleto s r. $\quad 16 T \alpha-$ $v \alpha 0 \xi \alpha \dot{\eta} \eta \xi] \tau \alpha \nu \alpha 0 \xi \alpha \dot{\alpha} \eta s$, ut supra.

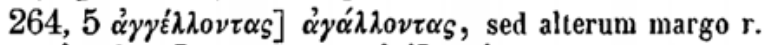

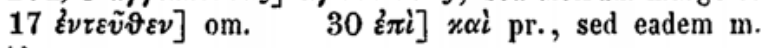
ut videtur correctum.

$265,19, \pi \varepsilon i \vartheta \varepsilon \tau \alpha \iota] \pi v ́ \vartheta \varepsilon \tau \alpha \iota$, sed $\varepsilon \iota$ margo r. 22

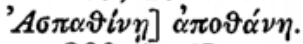

$266,1 \tau \omega]$ in rasura $25 \varepsilon v]$ supra v. ead. m. $\tau \dot{\alpha}]$ $\tau \tilde{n}$, sed $\tau \dot{\alpha}$ margo r. $26 \varepsilon \pi i] \pi \varepsilon \rho i$.

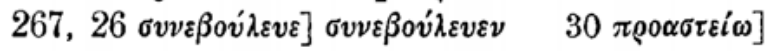
$\tau \omega$, $\pi \varrho \omega$, sed supra asteriscus $\mathbf{r}$.

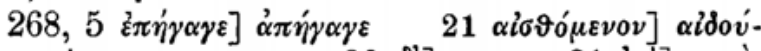
$\mu \varepsilon \nu 0 \nu$, alterum margo r. $\left.30 \delta^{\prime}\right]$ om. $\left.31 \varepsilon \pi i\right] \pi \alpha \rho \dot{\alpha}$.

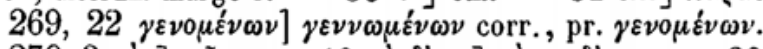
$270,3 \tau \dot{\partial} \nu] \tau \tilde{\omega} \nu$ pr. $18 \tau \dot{o} \delta \dot{\varepsilon}$ \%.] $\tau \dot{o}$ \%. $\delta \dot{\varepsilon}$ recte 28 $\delta ' \eta ै \delta \eta] \delta \dot{~}$.

$\left.271,1 \stackrel{\alpha}{\alpha} \alpha \pi 0 \lambda \circ \tilde{\nu} \nu \tau \sigma_{\varsigma}\right] \nu \alpha$ s. v. r. m. $\quad 31 \varepsilon v] \mathrm{om}$.

$\left.272,3 \delta \alpha \pi \alpha^{\prime} \nu \eta \nu\right] \delta \iota \alpha \pi \alpha^{\prime} \nu \eta \nu$ in ras., pr. fortasse $\delta \iota \alpha$ -

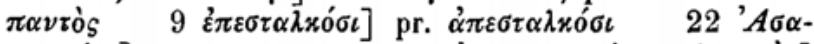

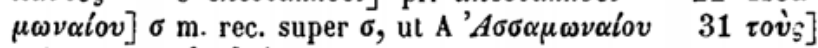

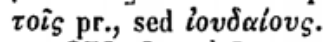

$273,3$ rovं $]$ om. $\left.\quad 11 \delta_{\dot{\varepsilon}}\right] \delta^{\prime}$ ut Wolfius $12 \mathrm{M \omega v}$ -

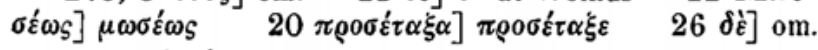

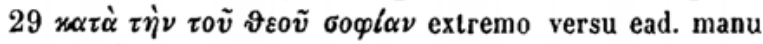
in marg. scripta.

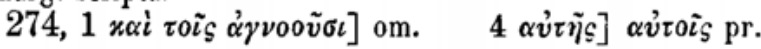


XXVIII

AD VOL. I.

$\left.\left.\pi \rho \dot{s}] \pi \alpha \rho \dot{\alpha} \quad 5 \delta^{\prime}\right] \delta \dot{\varepsilon} \quad x \alpha i\right]$ om. $27 M \omega \sigma \varepsilon \dot{\omega}$ ] $\mu \omega \sigma \varepsilon$ ç .

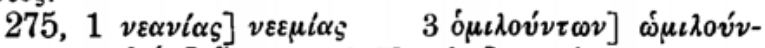

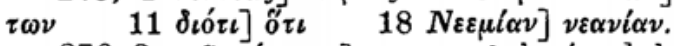

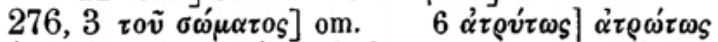

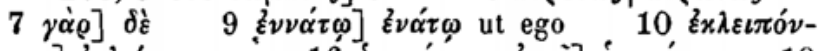

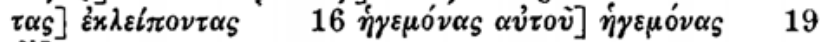
$\delta \dot{\varepsilon}] \mathrm{om}$.

277, $16 \pi \varepsilon \lambda \varepsilon x v \varphi o ́ \rho \omega \nu] \pi \varepsilon \lambda \varepsilon x \eta \varphi o ́ \varrho \omega \nu \quad 19 \delta v ́ \omega] \delta v_{0}$ $27 \mu \dot{\eta}]$ s. v. ead. m.

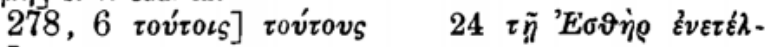
$\lambda \varepsilon \tau 0]$ om.

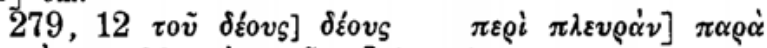
$\pi \lambda \varepsilon v \rho \dot{\nu} \nu 20 x \alpha i \pi \alpha \varrho \tilde{\eta} \sigma \alpha \nu]$ inter haec asteriscus $\mathrm{r}$.

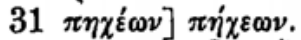

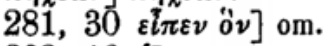

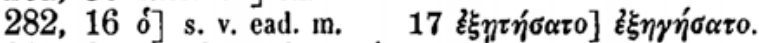

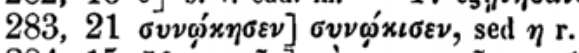

284, $15 M \alpha \nu \alpha \sigma \sigma \tilde{\eta} \nu] \tau \dot{\nu} \nu \mu \alpha \nu \alpha \sigma \sigma \tilde{\eta} \nu 17 \kappa \alpha i$ ó] $x \alpha i$.

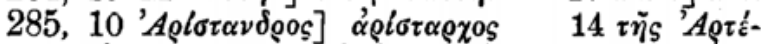

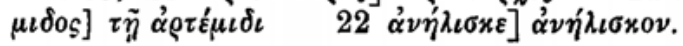

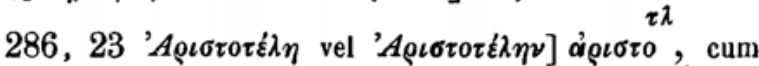
ductu scripto $\lambda$, qui $\lambda \eta$ videtur significare $z \pi \iota \sigma \tau o ́ \lambda \iota 0 \nu]$

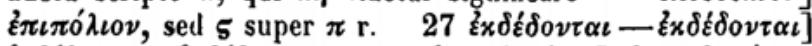

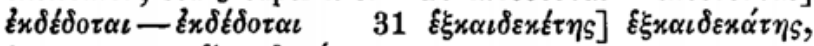
fere ut A, qui $\hat{\varepsilon} \xi x \alpha \iota \delta \varepsilon x \alpha ́$ rov.

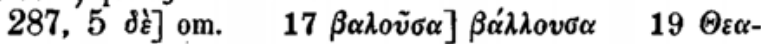

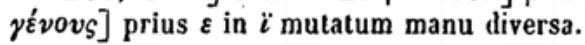

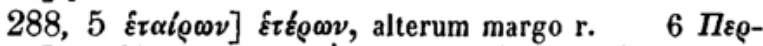
$\delta i x x o v] \pi \varepsilon \rho \delta i x o v \quad 29 \tau \dot{\eta} \nu \pi \alpha \rho \alpha \lambda i \alpha \nu] \pi \alpha \rho \alpha \lambda i \alpha \nu \quad 30$ $\left.x \alpha i K_{\iota} \lambda \iota x i \alpha \nu\right] \quad x \iota \lambda_{\iota x} i \alpha \nu$.

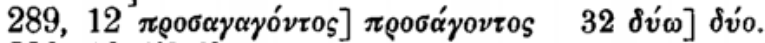
$290,19 \delta \dot{\varepsilon}] \delta^{\prime}$ ' ut Wolfius et ego.

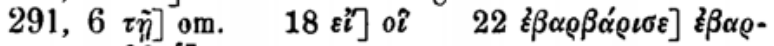

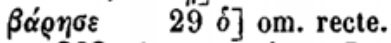

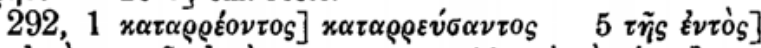

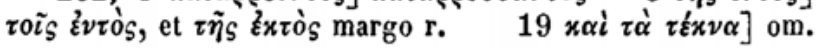


293, $11 \pi \varepsilon \delta i o \nu] \pi \alpha \iota \delta i o \nu$, sed $\pi \varepsilon$ margo r. $13 x \alpha i$

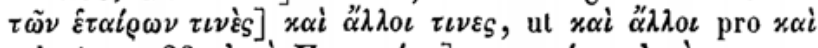
solo A $28 \varepsilon l \mu \dot{\eta} \Pi \alpha \varrho \mu \varepsilon v i \omega \nu] \pi \alpha \varrho \mu \varepsilon v i \omega \nu \varepsilon l \mu \dot{\eta}$.

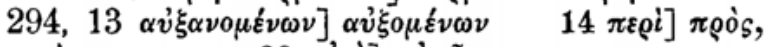

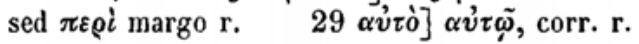

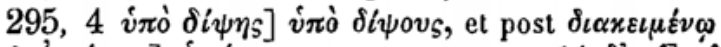

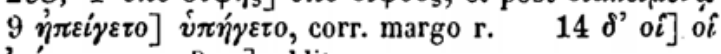
$30 \varepsilon x \operatorname{\varepsilon }_{\mu \iota \sigma \alpha \nu}$ om. Duc.] addit.

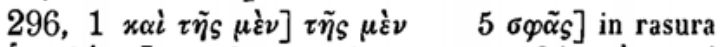
$\left.22 \dot{\eta} \tau \tau \eta \vartheta \varepsilon^{\prime} v \tau \alpha\right]$ ante hoc asteriscus r. $24 x \alpha i \mu \varepsilon \gamma \alpha \lambda \alpha v$ -

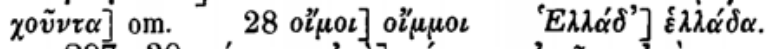

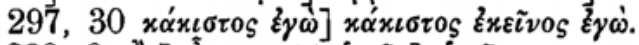

298, $9 \stackrel{\omega}{\omega} \nu] \stackrel{\tilde{\eta}}{\nu} \quad 11 \dot{v} \mu \tilde{\omega} \nu] \dot{\eta} \mu \tilde{\omega} \nu$.

299, $8 \delta v \sigma i] \delta v \sigma^{\prime}$, i. e. $\delta v \sigma i v$, ut Wolfius ỏvic̀s]

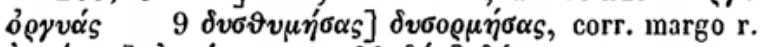

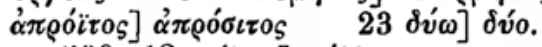

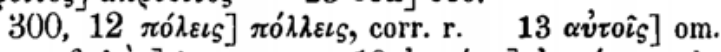

$17 \varkappa \alpha \tau \alpha \beta \alpha \lambda \dot{\omega} \nu]$ in rasura, $18 \dot{\varepsilon} \nu \mu \varepsilon \dot{\varepsilon \varepsilon l]} \varepsilon v \mu \varepsilon \dot{\varepsilon} \sigma \omega$, sed cum

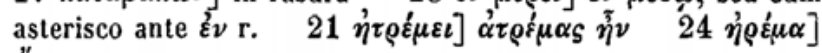

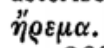

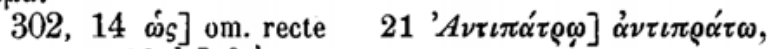
corr. r. $32 \quad \varepsilon x] \delta i \dot{\alpha}$.

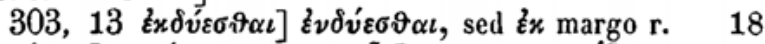

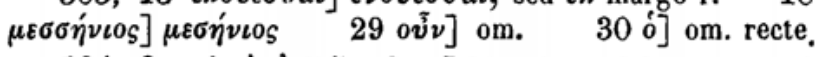

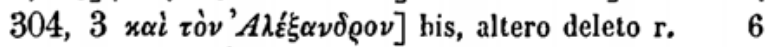
$\tau \lambda$

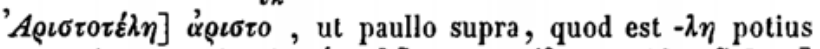

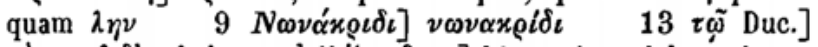

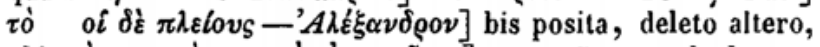
ubi $\varphi \dot{\eta}$ pro $\varphi \dot{\alpha} \mu \dot{\eta} \dot{\alpha} \pi 0 \sigma \tau \tilde{\eta} \nu \alpha \iota] \pi \rho 0 \sigma \tau \tilde{\eta} \nu \alpha \iota$, sed alterum margo $\mathrm{r}$.

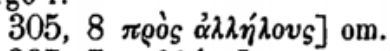

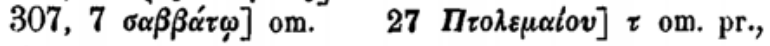
insertum $\mathrm{r}$.

309,5 o $\left.\beta \alpha \sigma \iota \lambda_{\varepsilon} \dot{v}_{S}\right] x \alpha i$ o $\left.\beta \alpha \sigma \iota \lambda \varepsilon \dot{v}_{S} \quad 8 x \alpha \lambda \lambda i \sigma_{\tau} \alpha_{S}\right]$

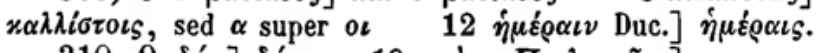
$\left.\left.310,9 \delta v^{\prime} \omega\right] \delta v^{\prime} \quad 19 x \alpha i-\Pi \tau 0 \lambda \varepsilon \mu \alpha \tilde{i} 0 \nu\right]$ om. 
$\left.311,17 \delta^{\prime}\right] \delta \dot{\varepsilon}$.

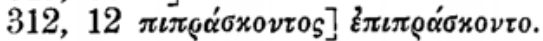

$313,26 \pi \rho \circ \varepsilon i \dot{\mu} \alpha] \pi \varrho 0 \sigma \varepsilon \tau i \mu \alpha$, deleto $\sigma \mathrm{r}$. $\tau \dot{\alpha}] \mathrm{om}$.

$314, \quad 8 \not ̋ \sigma \omega \nu]$ ő $\sigma \alpha \quad 16$ है $\emptyset \eta$ om. Duc.] addit

$315,23 \delta \dot{\varepsilon} x \alpha i] \delta \dot{\varepsilon}$.

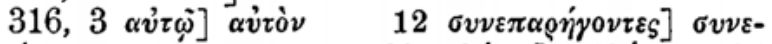

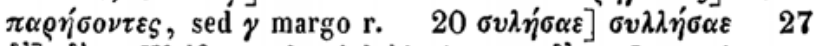
$\left.\delta^{\prime}\right] \delta \dot{\varepsilon}$ ut Wolfus et haud dubie A, non $\delta$, ut Ducangius.

317,18 rıs] om.

$318,5 \delta \dot{\varepsilon} x \alpha i] \delta \dot{\varepsilon}$.

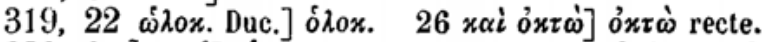

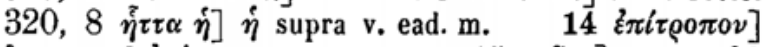

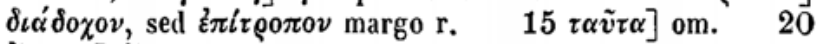

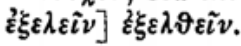

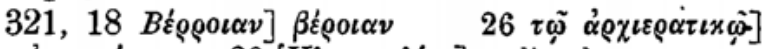

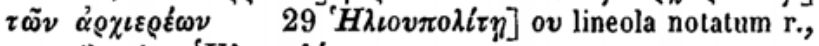
et scribendum 'H

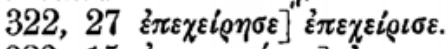

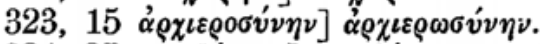

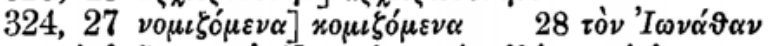

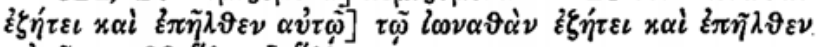

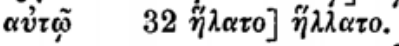

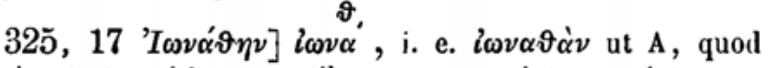
recipi potest, etsi in sequentibus quoque scriptura variat usque

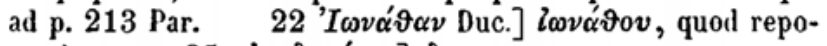

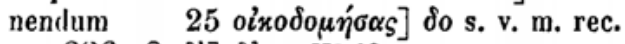

$326,8 \quad \delta \dot{\varepsilon}] \delta^{\prime}$ ' ut Wolfius et ego.

$327,6 \pi \alpha \rho \alpha \lambda \alpha \beta \dot{\omega} \nu] \lambda \alpha$ s. v. m. rec. $9 \pi \sigma^{\circ}$ Duc.]

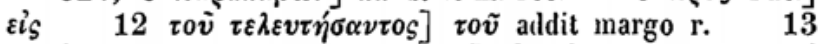

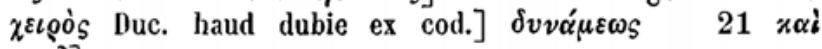
vixô] om.

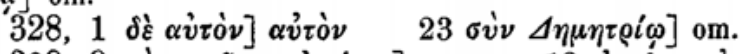

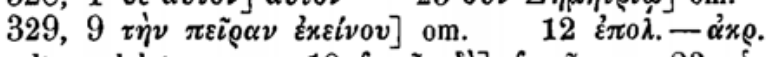

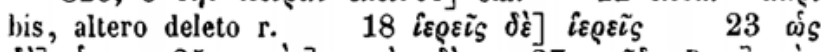

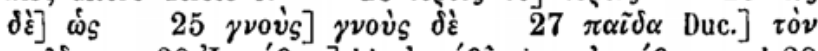

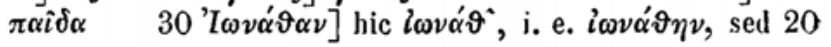




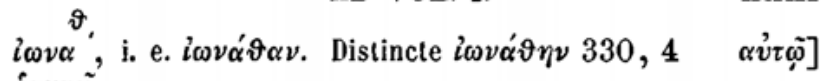
$\varepsilon \alpha v \tau \tilde{\omega}$.

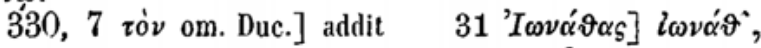

i. e. $i \omega v \alpha^{\prime} \vartheta \eta s$, sed $331,7,12 ; 332,22 i \omega v \alpha$, i. e. $i \omega v \alpha^{\prime}-$

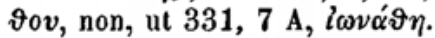

$331,8 \delta \dot{\varepsilon}]$ om. $12 \delta \dot{\varepsilon}]$ om. $\left.\quad \alpha v \tilde{v} \iota \varsigma \alpha v \dot{v} \tau \tilde{\iota}_{S}\right] \alpha \dot{v}-$

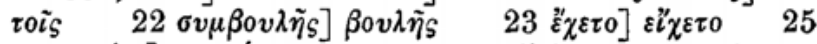
$\left.\sigma \alpha \tau \rho \alpha \pi i \alpha_{\varsigma}\right] \sigma \alpha \tau \rho \alpha \alpha_{\varsigma}$, hoc accentu, syllaba $\alpha_{\S}$ compendio expressa.

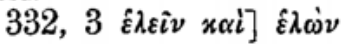
pendio expresso $\eta_{S}$.' Sic et 12

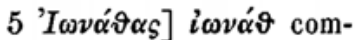

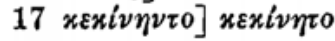

$25 \delta \eta \lambda \tilde{\omega} v]$ anguste inserit, qui pr. $\varepsilon \pi \varepsilon \mu \psi \varepsilon v$, omisso $\delta \eta \imath \lambda \omega ั \nu$.

$333,24 \dot{\alpha} \lambda \omega \mu \varepsilon \dot{\varepsilon} \nu 0 v] \alpha \lambda \omega \mu \varepsilon \dot{\varepsilon} v o v$, ut Wolfiana $25 \sigma \omega-$

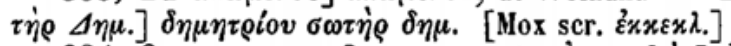

334, $3 \pi \rho 0 \sigma \varepsilon x$. Duc.] $\pi \rho 0 \varepsilon x . \quad 20 \dot{\alpha} \pi 0 \lambda \alpha \beta \dot{\omega} \nu] \alpha \pi 0 \lambda \alpha-$

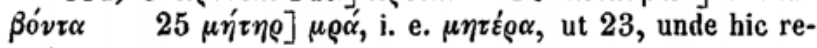
petitum. $\delta \varepsilon \dot{\xi} \alpha \tau$.

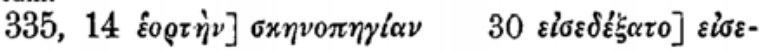

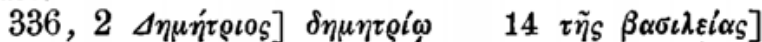

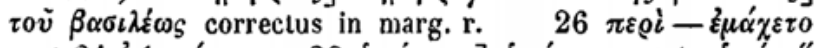

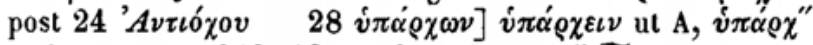

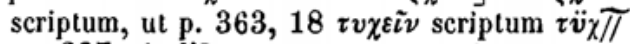

$337,1 \delta \dot{c}]$ om. estque aut delendum aut, ut ego feci,

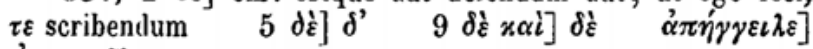
$\dot{\alpha} \pi \alpha \gamma \gamma \varepsilon i \bar{\lambda} \alpha \iota$.

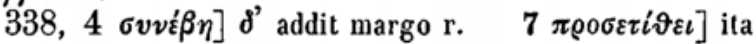
pr., quum super $\varepsilon$ praeter accentum appareat punctum ad $\iota$ pertinens, sed corr. $\pi \varrho \circ \sigma \varepsilon \tau \varepsilon \dot{\varepsilon} \eta \quad \mu \circ \varrho \varrho \alpha] \mu o \tilde{\varrho} \varrho \alpha$ pr., nunc $\mu о i \rho \alpha$.

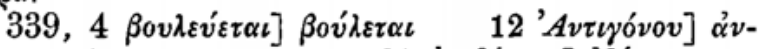

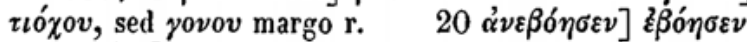

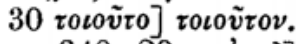

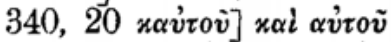

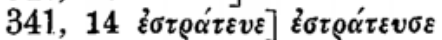

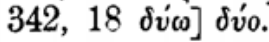

24 oi $[$ ois $\delta] \mathrm{om}$. $32 \varepsilon \varepsilon \varsigma] \omega s$. 


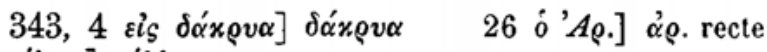
$\left.29 \mu \varepsilon^{\prime} \lambda \varepsilon \iota \nu\right] \mu \varepsilon^{\prime} \lambda \lambda \varepsilon \iota \nu$.

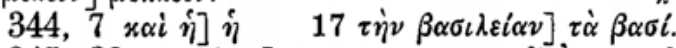

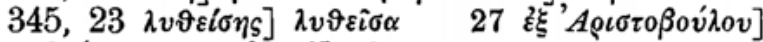

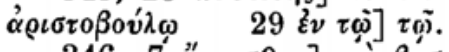

$\left.\left.346,7_{\alpha}^{\prime} \varrho \chi \varepsilon \sigma \vartheta \alpha \iota\right] \mu \eta_{\tilde{\sigma}}^{\prime} \beta \alpha \sigma \iota \lambda \varepsilon v \varepsilon \iota \nu \quad 16 \varepsilon i \sigma \eta^{\prime} \gamma \alpha \gamma \varepsilon v\right]$

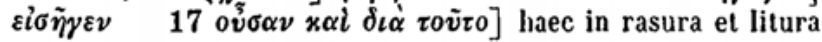
angustissimae lacunae ita scripta: $o v^{\sigma} \sigma$ (ita fere scribi solet $\alpha \nu$ $\tau \tau$

per compendium) $\delta \iota \alpha x \alpha i$.

$347,13 \gamma \circ \tilde{v} v] \gamma \dot{\alpha} \varrho$, sed $\delta \dot{\varepsilon}$ margo r. $\left.16 \delta^{\prime}\right] \delta \dot{\varepsilon}$

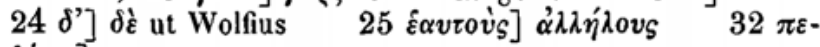
$\left.\lambda^{\prime} \dot{x} \varepsilon \iota\right]$ om.

348, 1 'P

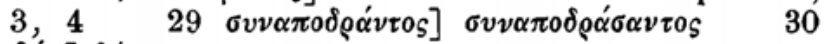
$\left.\delta v^{\prime} \omega\right] \delta v^{\prime}$.

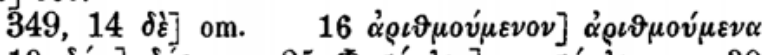

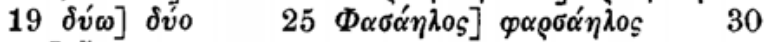
है $\pi \varepsilon \mu \psi \varepsilon]$ हैं $\pi \varepsilon \mu \pi \varepsilon$.

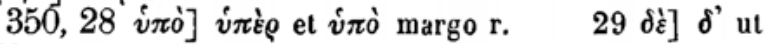
Wolfius.

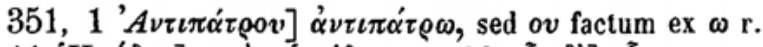

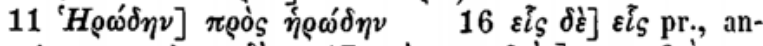
guste inserto ead. m. $\delta \dot{\varepsilon} 17$ $x \alpha i \pi \varepsilon \pi 0 \iota \vartheta \dot{\omega} s] \pi \varepsilon \pi 0 \iota \vartheta \dot{\omega} s$

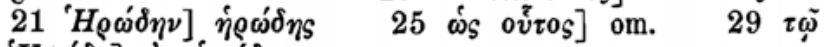

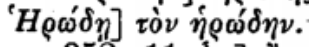

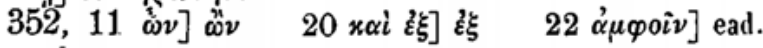
m. supplet margo.

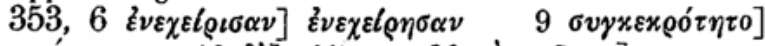

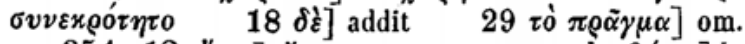

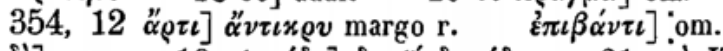

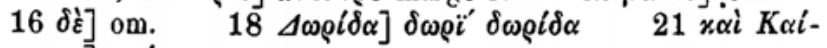

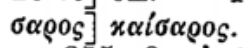

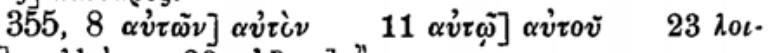

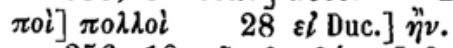

356, $10 \tau \tilde{\omega} \nu \beta \alpha \rho \beta \alpha ́ \rho \omega \nu] \beta \alpha \rho \beta \alpha ́ \rho \omega \nu \quad \delta \dot{\varepsilon} x \alpha i] \delta \dot{\varepsilon}$

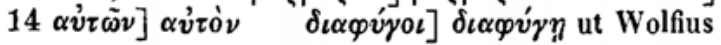


$\pi \alpha \rho \iota \pi \pi \dot{\alpha} \sigma \alpha \sigma \vartheta \alpha \iota] \pi \alpha \rho \iota \pi \pi \varepsilon \dot{v} \sigma \alpha \sigma \vartheta \alpha \iota$ ut Wolfiana. V. praef.

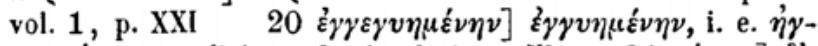

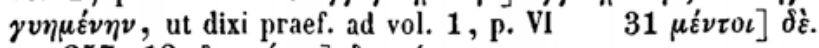

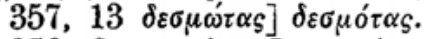

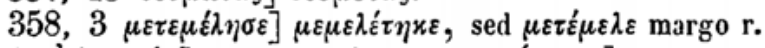

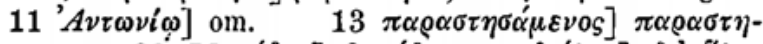

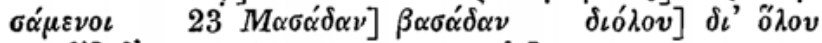
$\left.\delta_{\dot{\varepsilon}}^{\prime}\right] \delta^{\prime}$ ut Wolfius, recte $\left.24 \boldsymbol{\omega}_{\mathrm{s}}\right] \mathrm{om}$.

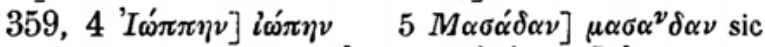

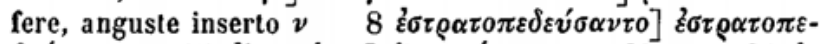

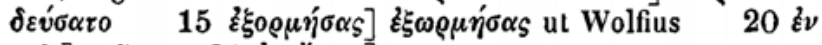

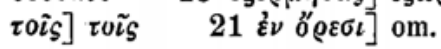

$360,7 x \alpha i \stackrel{\alpha}{\alpha} \pi \alpha \nu]$ x $x i$ ió $\tau \varepsilon \stackrel{\prime \prime}{\alpha} \pi \alpha \nu$, fere ut A, qui $x \alpha i$

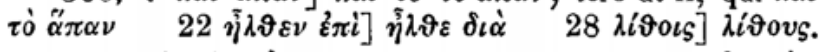

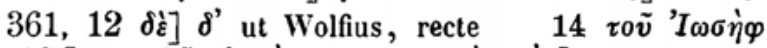

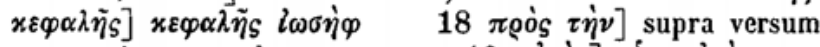

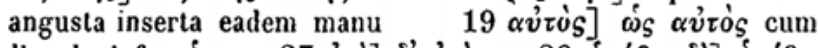

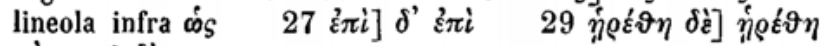
$\gamma \dot{\alpha} \varrho$, sed $\delta \dot{\varepsilon}$ margo r.

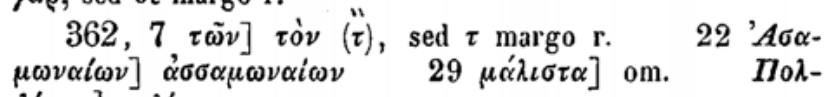
$\lambda i \omega \nu \alpha] \pi 0 \lambda i \omega \nu \alpha$.

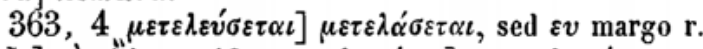

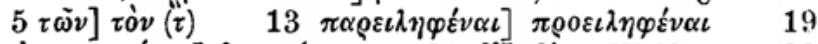

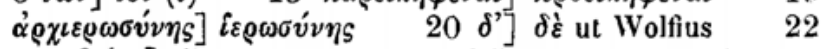
$\pi \varrho \varepsilon \sigma \beta \varepsilon i \alpha \nu]$ alia m. supra v., quae differt a manu marginis, et alia quoque nonnulla sic supplevit inter versus $23 x \alpha i$ $\delta \tilde{\rho} \propto \alpha$ है $\pi \varepsilon \mu \psi \varepsilon \varepsilon]$ om.

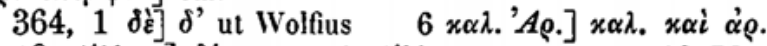

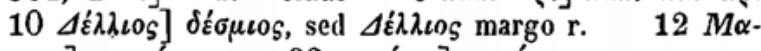

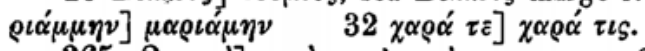

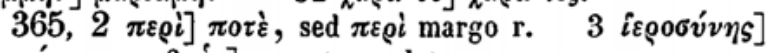

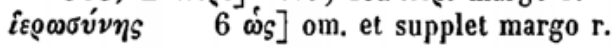

$\left.\begin{array}{lllll}365,7 & \delta \varepsilon^{\prime}\end{array}\right] \delta^{\prime}$ ut Wolfius et ego $\left.12 \quad \dot{\eta}\right] \ddot{\eta} \quad 16$

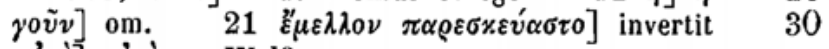
$\left.\alpha v^{\prime} \dot{o}^{\circ}\right] \alpha \dot{v} \tau \dot{v} \nu$ ut Wolfius.

$366,7 x \dot{\alpha} \nu]$ ] $x i$ ut A, cuius constructionis v. $\nu \eta^{\prime} \chi 0 \mu \alpha \iota$ ZONARAS v. 
exempla quaedam attuli ad Stephanum p. 1507, D; aliorum verborum cum dativo sic coniunctorum supra ad p. 228,3 . 367, $\left.\left.1 \quad \varepsilon^{2} x \varphi \dot{\eta} v \alpha \iota\right] \quad \varepsilon^{\prime} x \varphi \vartheta \tilde{\eta} v \alpha \iota \quad 3 \quad \delta \dot{\varepsilon} x \alpha i\right] x \alpha i$ s. v.

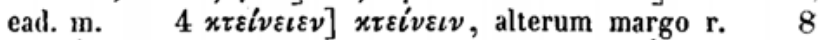

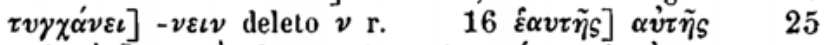

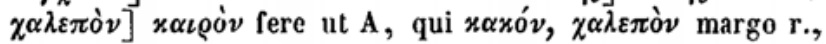
ut Ioscphus $15,3,9$.

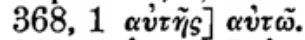

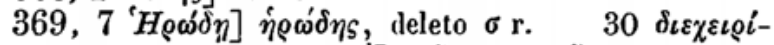

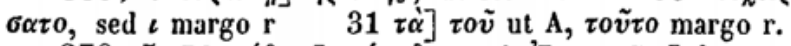

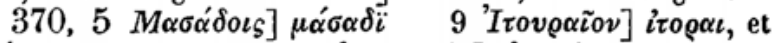

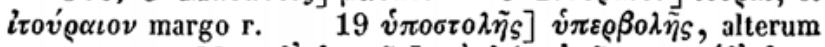
margo r. $\left.28 \mu \eta \delta^{\prime} \varepsilon \alpha v \tau \tilde{\omega} v\right] \mu \dot{\eta} \delta \varepsilon \dot{\xi} \alpha v i \tilde{\omega} \nu$, et $\mu \eta^{\prime} \delta^{\prime} \varepsilon \alpha v-$ $\tau \tilde{\omega} \nu$ margo $\mathrm{r}$.

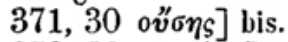

$\left.372,23 \vartheta \alpha \nu \alpha \tau \omega] \vartheta \alpha^{\prime} \nu \alpha \tau 0 \nu \quad 24 \varepsilon \alpha v \tau \tilde{\eta}_{S}\right] \alpha \dot{v} \tau \dot{\eta} \nu$, fere ut $A$, qui $\left.\hat{\varepsilon} \alpha v \tau \tilde{\eta} \quad 25 \varepsilon \kappa \pi \eta \delta \eta^{\prime} \sigma \alpha \sigma \alpha\right] \varepsilon^{2} \mu \pi \eta \delta \eta^{\prime} \sigma \alpha \sigma \alpha$ perspicue.

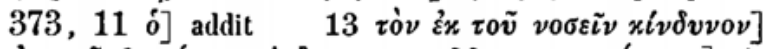

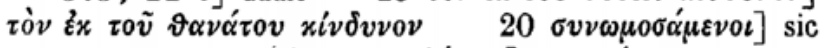

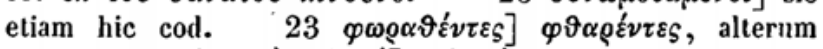
margo r. $27 x \alpha i \mu \varepsilon \lambda \iota \sigma \tau i] \mu \varepsilon \lambda \iota \sigma \tau i$.

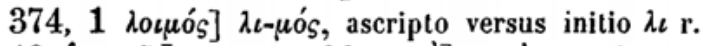

$13 \varepsilon \alpha v \tau o \tilde{\iota} \varsigma]$ om. $26 \pi \alpha \rho \dot{\alpha}] \pi \varepsilon \rho i$ perspicue, $\pi \ll \rho \dot{\alpha}$

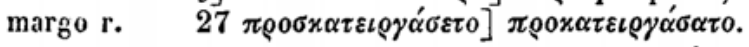

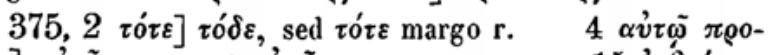
$x \varepsilon \chi.] \alpha \dot{v} \tau \tilde{\eta} \pi \rho 0 \sigma x$. et $\alpha \dot{v} \tau \tilde{\omega} \pi \varrho 0 x \varepsilon$ margo r. $15 \dot{\varepsilon} x \beta \alpha i v \varepsilon \iota \nu$

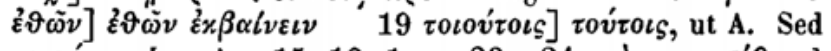

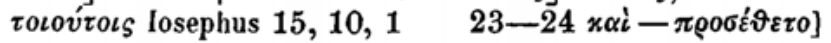
addit.

376, $7 \tau \alpha \dot{v} \tau \dot{\partial} \nu] \alpha \dot{v} \tau \dot{\partial} \nu$ et $\tau \alpha \dot{v} \tau \dot{v} \nu$ margo r. $\quad 11 \dot{\varepsilon} \xi \alpha v-$

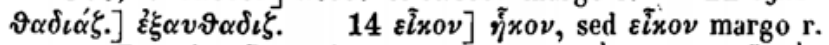

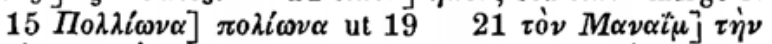

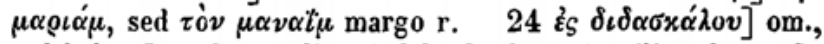
sed habet Iosephus : alioqui delenda foret inutilis ad $\varphi 0 \iota \tau \tilde{\alpha} \nu$ appendix.

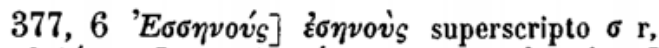

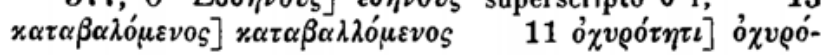


$\tau \alpha \tau o \nu$, et $\eta$ super $\alpha$ et $\iota$ super o r. et alia coniectura $\delta \iota \alpha \varphi o^{-}$ @os in marg. 28 ố $\tau$ ] ö $\tau \iota$, sed ôt $\tau \varepsilon$ margo r.

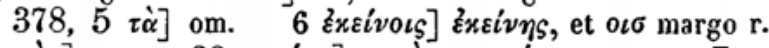
$\gamma \dot{\alpha} \varrho]$ om. $23 \mu \eta \tau \dot{\varepsilon} \rho \alpha] x \alpha \tau \dot{\alpha}$ et $\mu \eta \tau \varepsilon \dot{\varepsilon} \rho \alpha$ margo r. Tum addit $\tau \dot{\eta} v \mu \alpha \varrho \iota \dot{\alpha} \mu$ ut $\mathrm{A}$, quod omisso $\mu \eta \tau \varepsilon \underline{\varepsilon} \alpha$ habet Iosephus $16,1,2$.

$379,4 v \varepsilon \alpha v i \sigma x \omega \nu] \pi \alpha i \delta \omega \nu$, et margo eadem m., ut vi-

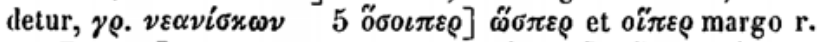

$\left.7 \tau \iota v \alpha_{S}\right] \omega \nu$ s. v. r. $\left.\tau 0 \tilde{\nu} v \nu \varepsilon \alpha \nu i \sigma x o \iota v\right] \tau \tilde{\omega} \nu \nu \varepsilon \alpha v i \sigma x \omega v$,

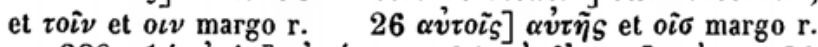

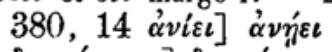

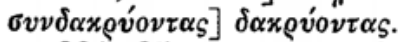

381, $25 \tau \dot{\eta} \nu \beta \alpha \sigma \iota \lambda \varepsilon i \alpha \nu] \tau \dot{\alpha} \beta \alpha \sigma i \lambda \varepsilon \iota \alpha$.

$382,2 \delta \dot{\varepsilon}] \delta^{\prime}$ ut A et Wolfius, recte

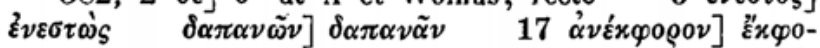

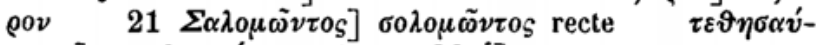

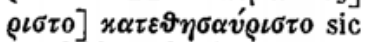

$383,16 \delta \varepsilon]$ om. 28 o] om.

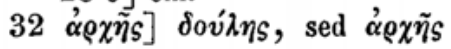
margo $\mathrm{r}$.

384, $4 \dot{\alpha} \pi \eta \gamma \gamma.] \varepsilon \pi \eta \gamma \gamma \gamma$., correcto $\dot{\alpha}$ r. , $8 \varepsilon i \pi \dot{\omega} \nu] \varepsilon \hat{\varepsilon}-$ $\pi 0 v 13$ ov่] supplet margo $\mathrm{r}, \mu \dot{\eta}$ pro ov A $17 \sigma v \nu$ $\vartheta \varepsilon \tilde{\imath} \nu \alpha \iota] \sigma v \mu \pi \varepsilon i \sigma \varepsilon \iota \nu$, et $\sigma v \nu \vartheta \varepsilon \tilde{v} \alpha \iota$ margo $\mathrm{r}$.

$\left.385,4{ }^{\circ} H \varrho \omega ́ \delta \eta \nu\right] \dot{\eta} \varrho \omega ́ \delta \eta \nu$ et $\nu$ s. v. r. $\sigma \alpha \nu] \varepsilon i \delta \varepsilon i \varepsilon \iota \sigma \alpha \nu 18 \pi \alpha \rho \dot{\alpha}] \pi \varepsilon \rho i$, et $\pi \alpha \rho \dot{\alpha}$ margo r.

$386,4 x \alpha \tau \alpha \sigma x.] x \alpha \tau \varepsilon \sigma x . \quad 5 \tau \dot{\eta} \nu \tau \tilde{\omega} \nu] \tau \tilde{\omega} \nu$

$14 \varepsilon i \delta \varepsilon i \eta-$ 22 $\left.\delta \iota \alpha \lambda v^{\prime} \varepsilon \imath \nu\right]$ om. et $\delta \iota \alpha \lambda v^{\prime} \sigma \varepsilon \nu$ (sic) margo r. $23 \chi \alpha \lambda \varepsilon \pi o^{\prime} \tau \eta-$ $\left.\tau{ }_{0 \varsigma}\right] \chi \alpha \lambda \varepsilon \pi \tau \sigma \tau \dot{\alpha} \tau \eta \varsigma$, et $\tau \eta \tau \sigma o \sigma$ margo r., sed alia, ut videtur, $\mathrm{m}$.

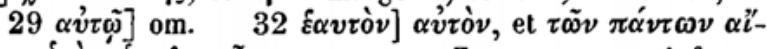

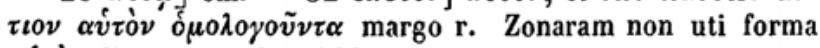
$\alpha \hat{v} \tau \grave{\nu} \nu$ dixi supra ad p. 160, 5.

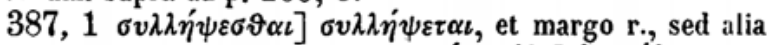
quam ad p. 387, 32 manu $\sigma \vartheta \quad 3$ 'H

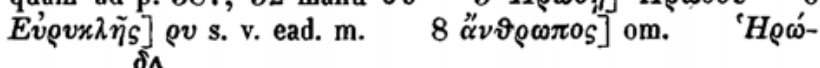
$\delta \eta \nu] \dot{\eta} \varrho \omega$ i. e. $\dot{\eta} \varrho \omega \delta \delta$. V. p. $364,1620 \pi 0 \iota \tilde{\eta} \sigma \alpha \iota$ ta-

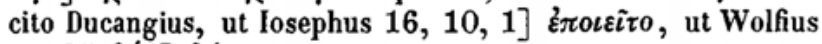
$\left.25 \delta v^{\prime} \omega\right] \delta v^{\prime}$.

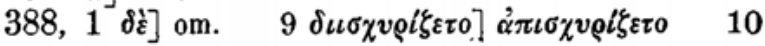



ead. $m$.

$389,6 \mu \varepsilon \dot{v} \tau \imath] \mu \varepsilon \dot{v} v \tau o \iota$, deleto o r. $10 \pi \alpha \rho \alpha \lambda \alpha \beta o ́ v \tau \alpha]$

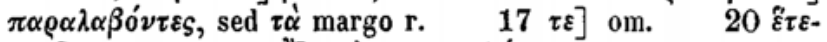
@ov] om. recte $\left.\eta^{\prime \prime}\right] x \alpha i$, unde $\pi \lambda \eta^{\prime} v$ margo r. $26 \pi \varepsilon$ $\left.\varrho \iota \varepsilon \sigma \tau \tilde{\omega} \tau \alpha_{S}\right] \pi \varrho 0 \varepsilon \sigma \tau \tilde{\omega} \tau \alpha_{S}$, sed $\varepsilon$ et $\iota$ s. v. r.

390,10 vंż̀े] $\pi \alpha \varrho$, sed $\pi \varepsilon \rho i$ margo r. 13 ỏvó$\mu \alpha \tau \iota]$ oै $\nu \rho \mu \alpha \quad 14 \pi 0 \lambda \lambda \dot{\alpha}]$ addit $\delta \dot{\varepsilon}$, quod delet margo r. $\left.15 \delta^{\prime} \dot{\varepsilon}\right]$ om.

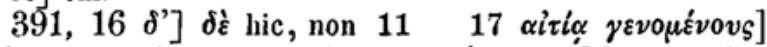

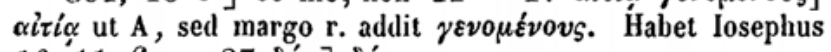
$\left.16,11,6 \quad 27 \delta v^{\prime} \omega\right] \delta$ júo.

$\left.392,3 \delta \eta \lambda \alpha \delta \eta^{\prime}\right] \delta \eta$ prius in ras. ead. $\left.\mathrm{m} . \quad 8 \pi \alpha i \delta \varepsilon \varepsilon\right]$

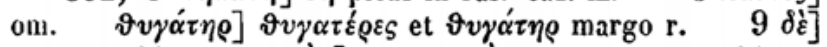

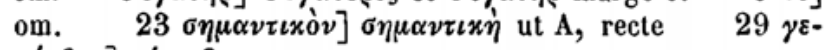

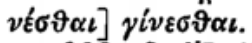

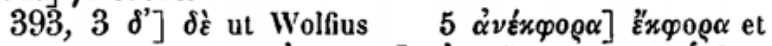

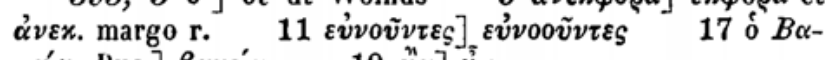
$\gamma \omega^{\prime} \alpha_{S}$ Duc.] $\left.\beta \alpha \gamma \omega \alpha_{S} \quad 19 \omega \nu\right] \eta^{n} \nu$.

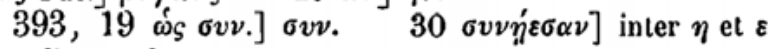
deleta littera, fortasse $\varrho$.

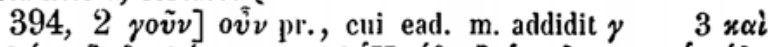

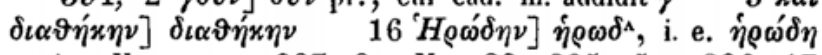
ut A. V. supra p. 387,8 . Mox $23 ; 395,5$ et 396,17

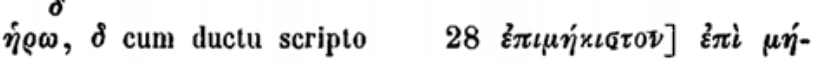

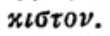

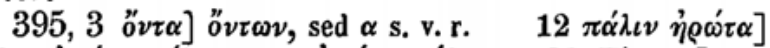

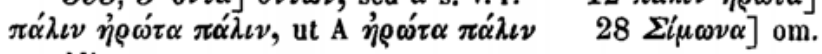
et addit margo $r$.

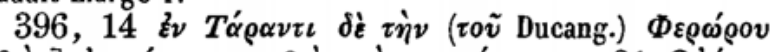

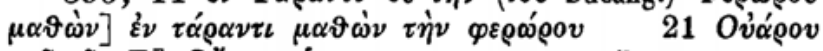

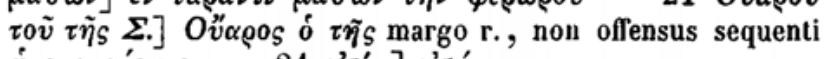

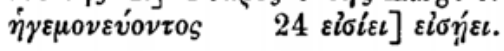

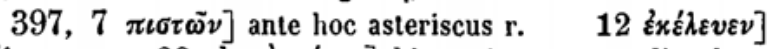

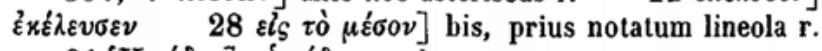

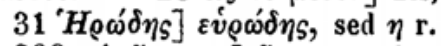

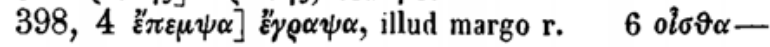


$\gamma \nu \omega \sigma \vartheta \varepsilon i \eta \nu]$ om. ut A, addit margo r., ubi $\delta \dot{v} \omega 14$ ' $A x \mu \grave{\eta}$

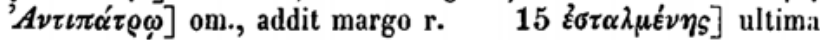
superscripta sic ut $\eta$ videatur, unde ad $\tau \tilde{\eta} S$ praecedens $\tau \tilde{\eta}$ margo r. 18 $\quad 1 \alpha \tau \dot{\alpha} \tau o \tilde{v}]$ $x \alpha \tau \dot{\alpha} \sigma o \tilde{v}$.

$\left.399,1 \delta^{\prime}\right]$ om. $\left.\quad 11 \delta^{\prime}\right] \delta$ \&े 12 है $\left.v\right]$ om. $\quad 20$

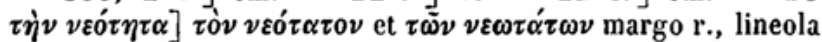

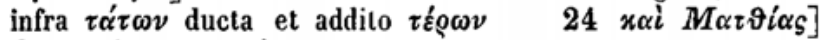
$\delta \mu \alpha \tau \vartheta i \alpha \mathcal{S}$, sed $x \alpha i$ margo $\mathrm{r}$.

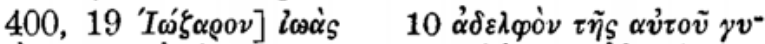

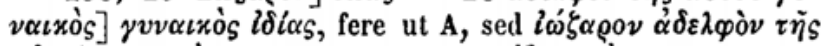

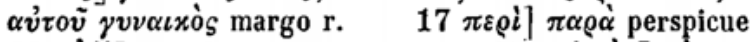

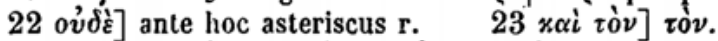

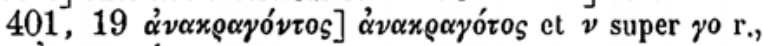
ut A $\dot{\alpha} \nu \alpha x \rho \alpha \gamma o ́$ tos.

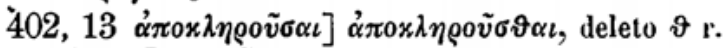

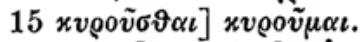

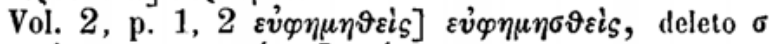

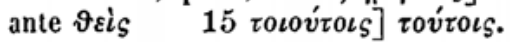

$2,3 \pi \alpha \rho \alpha \chi \rho \tilde{\eta} \mu \alpha]$ quum $\pi \alpha \rho \alpha$ scriptum sit, ut $\pi \varepsilon \rho i$ vi-

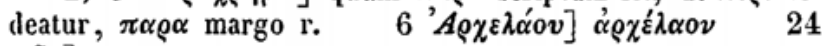
$\tau \tilde{\omega} v] \mathrm{om}$.

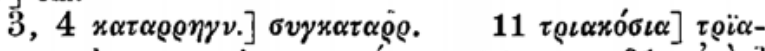

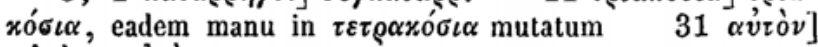
$\alpha \dot{v} \tau \dot{\alpha}$ et $\alpha \dot{v} \tau \dot{\nu} \nu$ margo $\mathrm{r}$.

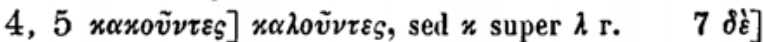

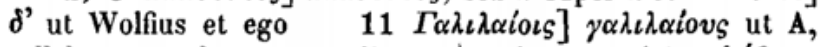
syllaba ov $v_{\varsigma}$ eodem compendio superscripta quo 4 in $\pi \lambda \eta^{\prime} \vartheta 0 v \varsigma$

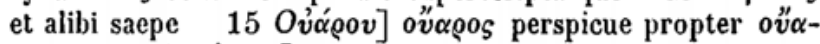
९os $723 \pi \varepsilon \dot{\varepsilon} \mu \varepsilon \iota]$ om.

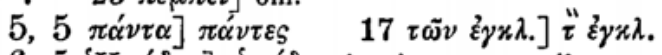

6, $\left.5{ }^{~ ' H \varrho \omega ́ \delta} \eta \nu\right]$ ¿’

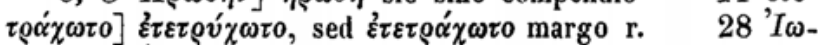

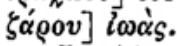

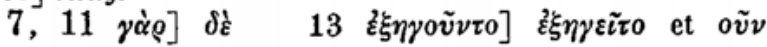
margo $\mathrm{r}$.

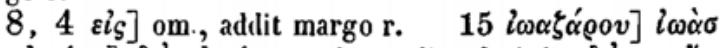

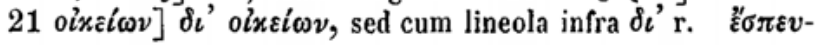


$\delta o v]$ o $v$ in rasura, sed eadem manu scriptum. A tamen $\tilde{\varepsilon}^{\prime \prime} \sigma \pi v \delta \delta \varepsilon$, quod non sufficit spatio inter hoc et $x \varepsilon \rho \delta \tilde{\nu}$.

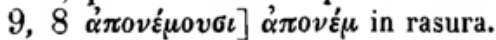

$10,3 \dot{\eta}] \tau \tilde{\eta}$, sed cum lineola infra hoc r. $6 x \alpha i$

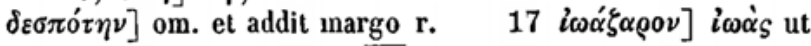

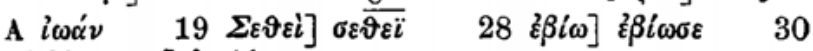

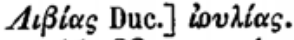

11, $22 \pi \varepsilon \rho \iota \sigma \tau \dot{\eta} \sigma \alpha \varsigma] \pi \varepsilon \rho \iota \vartheta \tilde{\eta} \nu \alpha \iota$, sed $\pi \varepsilon \rho \iota \sigma \tau \dot{\eta} \sigma \alpha \varsigma$ margo r. $\omega$

$\left.23 \vartheta \alpha^{\alpha} \nu \alpha \tau \nu \nu\right] \vartheta \alpha \nu \alpha^{\prime}$, sed $\vartheta \alpha \dot{\alpha} \alpha \tau o \nu$ margo r.

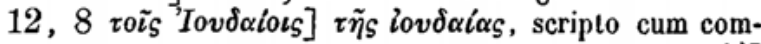
pendio $\alpha \mathcal{S}$, sed oi margo r., non animadverso altero $17 \quad \delta \dot{\eta}]$ $\dot{\varepsilon}$ initio versus, nec littera apparet detrita.

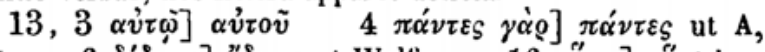

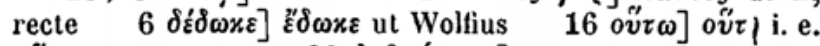

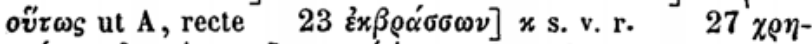

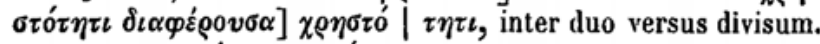

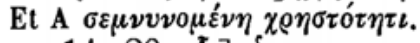

$14,20 \alpha i s] \omega_{s}$.

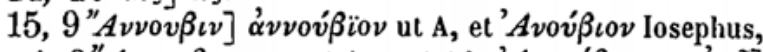

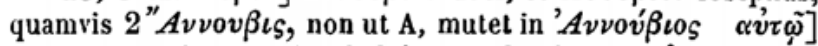
etiam hic codex, ut haud dubie scribendum sit દ̇ $\mu \alpha v \tau \omega \omega^{\prime}$, ut apud Iosephum. Nam quod Pinderus nescio an ex A posuit $\alpha v \tau \tilde{v}$, iam ad p. 160, 5 animadverti non dicere Zonaram

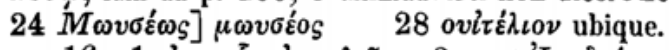

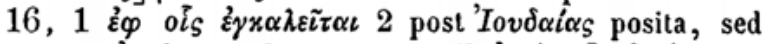
pr. m. post $\alpha \pi 0 \lambda$. transferuntur 17 Ėxcivov] oixciov

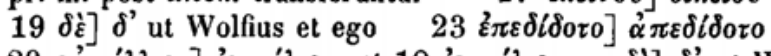

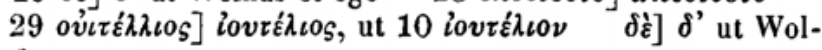
fius, recte.

$17,18 \delta \dot{\varepsilon}] \delta \dot{\varepsilon} \delta$.

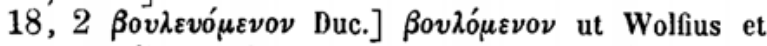
ego, neque aliter, opinor, A, quamvis taceat collatio apud Pinderum, qui $\beta o v \lambda \varepsilon v o ́ \mu \varepsilon v o v$, ut ad alia Parisinae vitia. Conf. autem p. 24, 1822 ह่ $\left.\delta \varepsilon^{\prime} \sigma u v_{S} 28 \delta \delta^{\prime} \xi \alpha \nu\right] \delta \delta^{\prime} \xi \alpha, \nu$ paullo supra ead. $\mathrm{m}$.

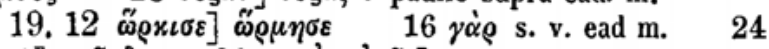
$\tau \tilde{\eta}$ 4.] $\left.\tau 0 \tilde{v} \delta . \quad 26 \pi \alpha \rho{ }^{\prime} \alpha \dot{v} \tau \tilde{\eta}_{S}\right]$ om. 


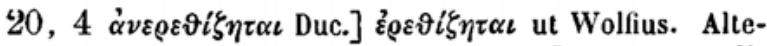
rum losephus $18,6,1$. Itaque hoc quidem Ducangius ex li-

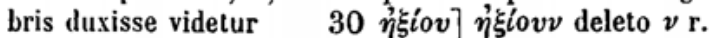

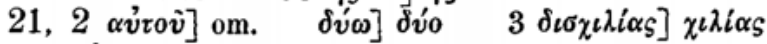
ut A, sed $\delta \iota \sigma$ margo r. $\chi \iota \lambda i \alpha \iota \varsigma$ etiam losephi $18,6,3$ codex Busb., qui etiam paullo ante solus cum Zonara Mér@ov pro

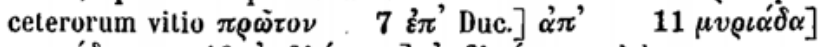

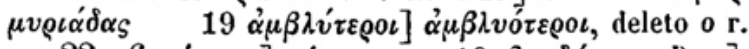

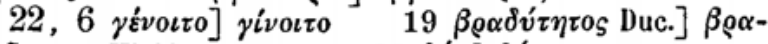
$\delta v \tau \tilde{\eta} \tau o s$ ut Wolfius et ego $\left.20 \delta v^{\prime} \omega\right] \delta$ dvo.

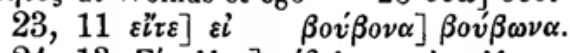

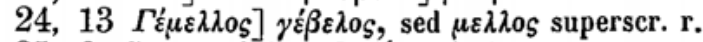

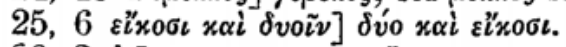

$\left.26,2 \varepsilon^{\prime} \nu\right]$ in rasura $\left.\tau 0 \tilde{v}\right]$ om. ut A. Iosephus 18, 6,

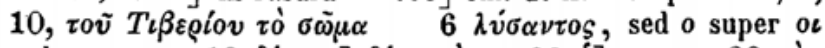

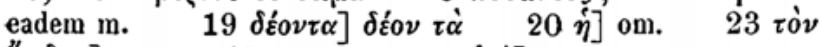
$\not \nu \delta \varrho \alpha]$ om. et addit margo r.

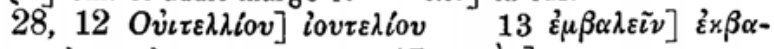

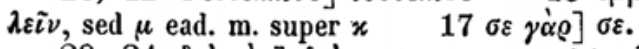

$29,24 \delta \iota \dot{\alpha} \tau \dot{\omega} \nu] \delta \iota \dot{\alpha}$ ut A, recte $31 \tau \tilde{\omega} \nu \pi \varepsilon \rho i \sigma \varepsilon \alpha v$ -

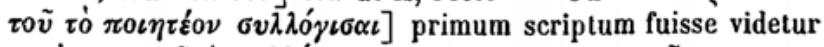

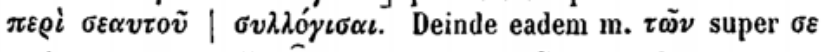
scriptum compendio $\tilde{\tau}$, et post $\sigma \varepsilon \alpha v$ rov supra finem versus eminens tò et prominens supra initium sequentis versus

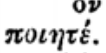

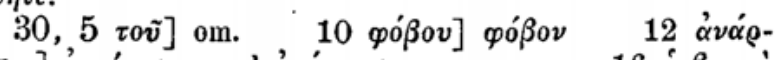

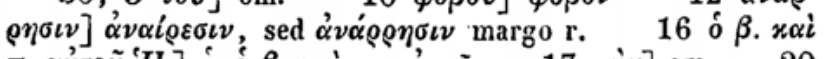

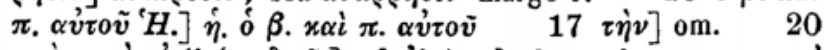

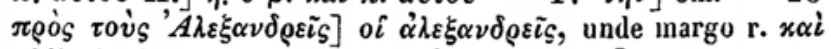
addit initio seq. versus ante oi 27 ioi $\nu] \nu$ in ras., pr. fortasse $c$ ( $\tau \sigma \tilde{i} \mathrm{~s})$.

$\left.31,19 \delta v^{\prime} \omega\right]$ dv́o $\left.22 \delta \xi\right] \delta$ ut Wolfius et ego 25

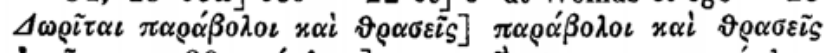

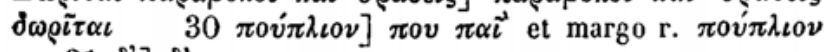
$\left.31 \delta^{\prime}\right] \delta \dot{\varepsilon}$.

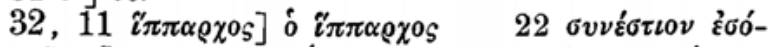

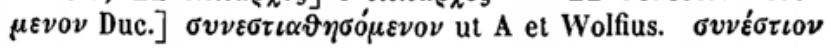


દ̇бó $\mu \varepsilon v o v$ unde petiverit Ducangius non dixit. Iosephus $\sigma v \nu$ -

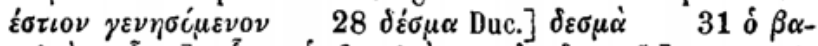

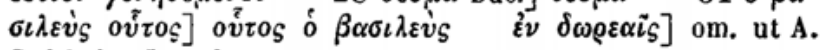
Sed habet Iosephus.
$33,2$ ó $\mu$ oi $\omega s]$ om.

$\left.34,1 \tau 0 \hat{i}_{S}\right] \tau \check{\eta}_{S}$

$\pi \dot{\omega} \nu] x \alpha \tau \varepsilon \dot{\varepsilon} \iota \tau \varepsilon \varepsilon$.

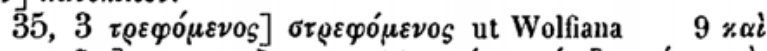

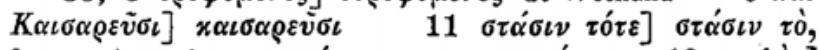
fere ut $\mathrm{A}$, qui recte $\sigma \tau \alpha \dot{\sigma} \sigma \nu \tau \varepsilon$, et s. v. $\tau$ ó $\tau \varepsilon$

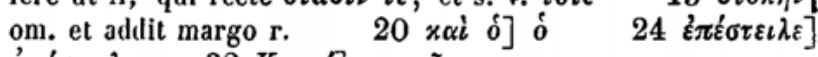

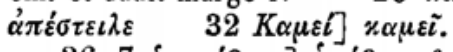

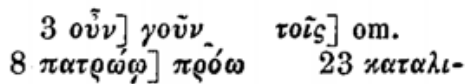

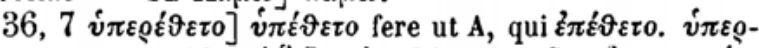

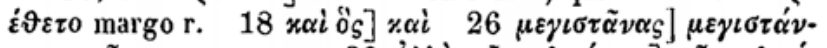
$\tau \alpha S$ et $\tilde{\alpha} \nu \alpha \sigma$ margo r. $\left.29 \dot{\alpha} \lambda \lambda \dot{\alpha} \tau \tilde{\omega} \nu \pi \lambda \varepsilon \iota \sigma^{\prime} \omega \omega \nu\right] \tau \tilde{\omega} \nu \pi \lambda \varepsilon \iota 0^{-}$ $\nu \omega \nu$ fere ut A, qui $\tau \tilde{\omega} \nu \pi \lambda \varepsilon \iota v^{\prime} \omega \nu \nu \delta \varepsilon^{\prime}$. Sed $\alpha \lambda \lambda \dot{\alpha}$ addit margo

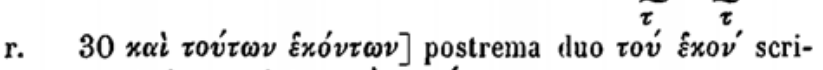
pta anguste inserta inter $x \alpha i$ et $\tau \eta^{\prime} v$.

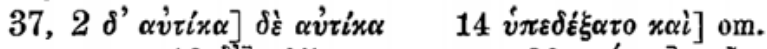

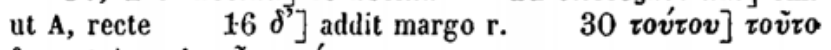

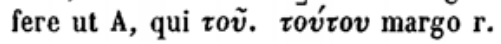

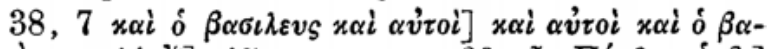

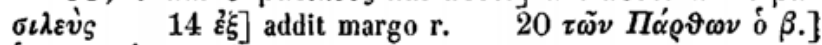
o $\tau \tilde{\omega} \nu \pi \alpha \dot{\alpha} \vartheta \omega \nu \beta$., ut Wolfiana. Quod praetuli. Iosephus 20,

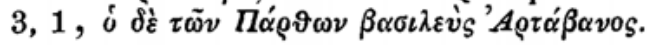

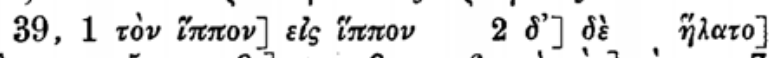

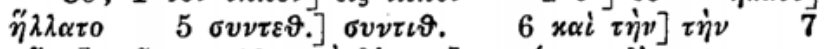

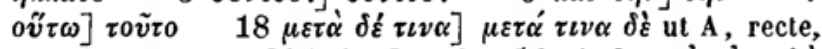
etsi alterum est p. 285, 3. Iosephus $10,4,3 \mu \varepsilon \tau$ ' ov $\pi 0 \lambda \dot{v}$

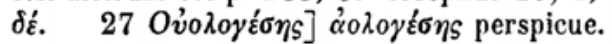

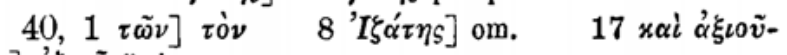
$\sigma \iota v] \dot{\alpha} \iota\llcorner\tilde{v} \sigma \ddot{i}$ sic.

41, $6 \tau \dot{\eta} \nu \delta^{\prime} \alpha \varrho \chi \dot{\eta} \nu \rightarrow \varkappa \alpha \tau \varepsilon^{\prime} \lambda \iota \pi \varepsilon \nu$ Duc. ex codd.] om. ut A et Wolfus $\left.13 \imath^{\prime \prime} \lambda \eta \nu\right] \iota^{\prime \prime} \lambda v \nu$.

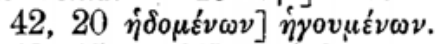

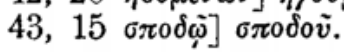




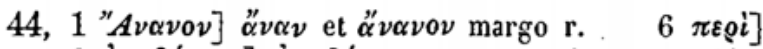
om. $\left.\quad 9 \alpha_{\alpha}^{\prime} \nu \alpha \beta \dot{\alpha} \nu \tau \alpha_{\varsigma}\right] \alpha^{\alpha} \nu \alpha \beta \alpha^{\prime} \tau \alpha_{S}$ et $\nu$ s. v. ead. m., ut videtur $\left.14 \Phi_{\eta}^{\prime} \lambda \iota x \alpha\right] \varphi^{\prime} i \eta \eta x \alpha$ et sic infra, sed $\varphi \eta^{\prime} \lambda \iota x \alpha$ margo r.

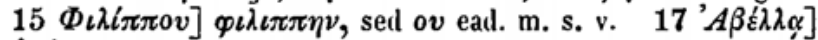

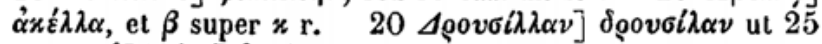

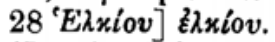

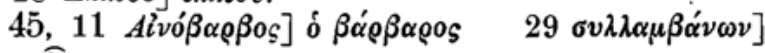
$\sigma \alpha \lambda \alpha \mu \hat{\alpha} \nu$, sed asterisco notatum et lineola infra ducta $r$.

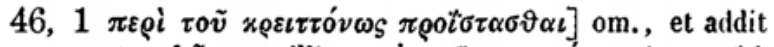

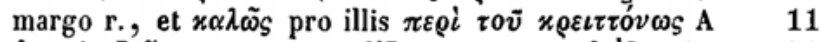

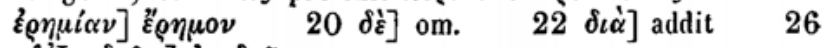

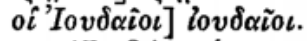

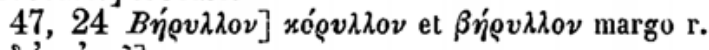
$25 \delta i$ ' $\alpha \dot{v} \tau o v i] \mathrm{om}$.

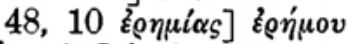

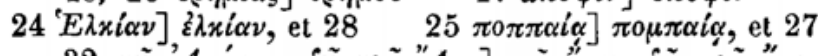

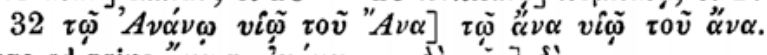
Margo ad prius ${ }^{\prime \prime} \nu \dot{\alpha}$ r. $\left.\dot{\alpha}^{\prime} v \alpha^{\prime} v \omega, \delta \dot{\varepsilon} \tau \tilde{\omega}^{\prime} v\right] \delta \dot{\varepsilon}$.

$49,21 \Gamma \alpha \mu \alpha \lambda \iota \dot{\eta} \lambda] \gamma \alpha \beta_{\rho}^{\prime} \iota \dot{\eta} \lambda$ hic et 50,4 .

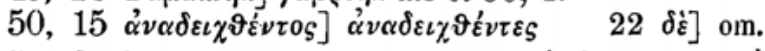

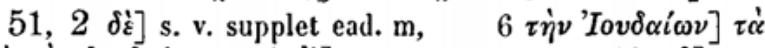

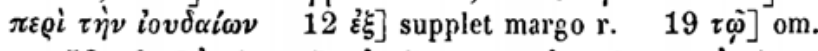

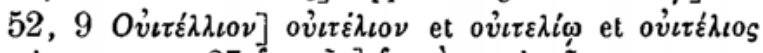
etiam in seqq. $\quad 27 \hat{\varepsilon} \alpha v \tau \tilde{\omega} \nu] \hat{\varepsilon} \alpha v \tau \dot{0} \nu$, sed $\tau \tilde{\omega} \nu$ margo r.

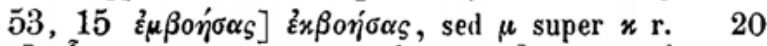

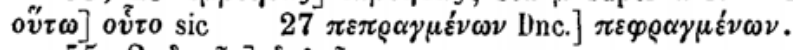

$55,2 \delta \eta \circ \tilde{v} v] \delta \eta \lambda o \tilde{v} \nu$.

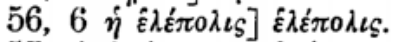

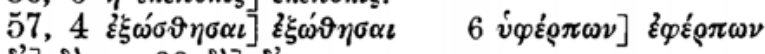
$\left.\left.26 \delta^{\prime}\right] \delta \dot{\varepsilon} \quad 30 \delta \dot{\varepsilon}\right] \delta^{\prime}$.

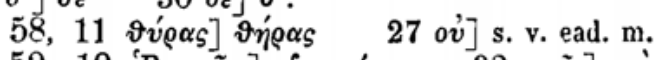

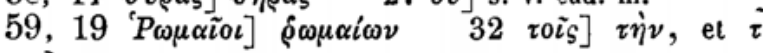
i. e. $\tau \dot{\omega} \nu$ margo $\mathrm{r}$.

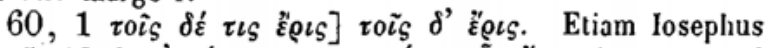

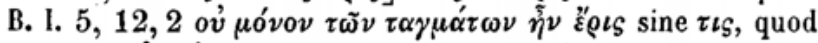
est post $\delta \varrho \mu \eta े$ apud utrumque. Sel codex similiter peccat p. 61, $\left.25 \alpha v \hat{o}^{\prime}\right]$ om. $\left.11 \% \alpha i \nu \varepsilon \alpha v i \alpha \iota\right] v \varepsilon \alpha v i \alpha \iota$, sed 


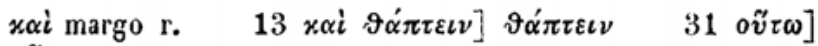
oút $\omega_{s}$,
$61,1 \delta \dot{\varepsilon} \tau \iota] \delta \dot{\varepsilon}$
$12 \dot{\alpha} \nu \alpha \sigma \chi \iota \sigma \vartheta \tilde{\eta} \nu \alpha \iota] \sigma \chi \iota \sigma \vartheta \tilde{\eta} \nu \alpha \iota$

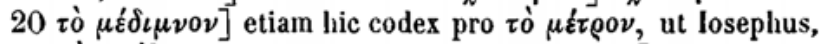
vel $\tau \dot{o} \nu \mu \varepsilon \dot{\delta} \iota \mu \nu 0 \nu$, ut alii omnes $22 \pi 0 \eta \lambda 0 \gamma \varepsilon \tilde{\imath} \nu] \pi 0 \lambda v \lambda o \gamma \varepsilon i \nu$,

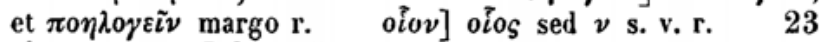

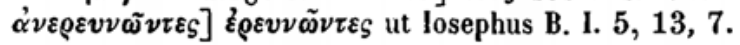

$62,10 \dot{\alpha} \nu \delta \rho i \alpha \nu$ Duc.] $\alpha \nu \delta \rho \varepsilon i \alpha \nu 13 \pi \alpha \rho \varepsilon \sigma \hat{v} \eta \eta \sigma \alpha v]$

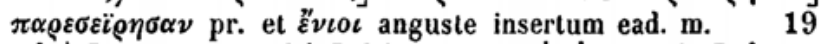
$\alpha \dot{v} \tau \dot{0} \nu]$ om. $\left.\left.25 \delta v^{\prime} \omega\right] \delta v_{0} \quad 26 \tau \dot{\eta} \nu ' A \nu \tau \omega \nu i \alpha \nu\right] \alpha \nu \tau \omega-$ ví $\alpha \nu \quad 28 \delta \dot{\varepsilon}]$ om.

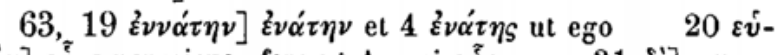

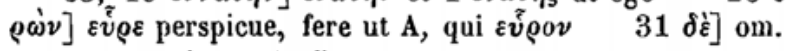

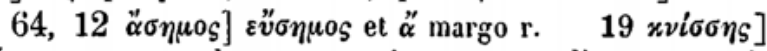
$\varkappa v i \sigma \eta s$, ut ego, sed super $\sigma$ scripto $\sigma$ manu diversa et antiquiori quam quae in marg. conspicitur $\left.\pi \varrho 0 \sigma \beta \alpha \lambda \lambda_{0} v_{\sigma} \eta \mathrm{s}\right]$

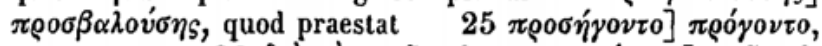

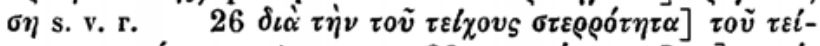

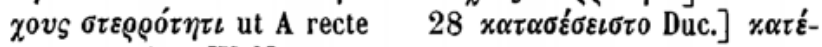

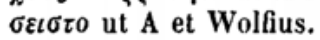

$65,2 \beta \lambda \alpha^{\prime} \beta \eta_{s}$ Duc.] $\beta \lambda \alpha \dot{\beta} \eta \nu$ ut A et Wolfius, quod quum sit etian in losephi nonnullis $6,4,1,9$, restitui pro eo quod Ducangius incertum unde intulerat. Est idem infra $23 \quad 3$

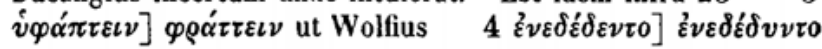

$6 \delta \dot{\varepsilon}]$ om. $\delta$ ' Wolfius et ego 29 है $\xi \omega$ infra versum paginae ultimum supplet ead. $\mathrm{m}$.

66, $8 \delta \iota \alpha \rho \pi \alpha^{\prime} \sigma \alpha \iota$ Duc.] $\dot{\alpha} \varrho \pi \dot{\alpha} \sigma \alpha \iota$.

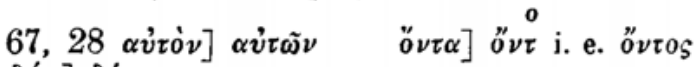

$\left.\delta v^{\prime} \omega\right] \delta v^{\prime}$.

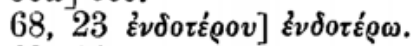

$69,14 \pi \alpha \rho \alpha x \lambda i \nu \omega \nu] \pi \alpha \varrho \varepsilon \gamma x \lambda i \nu \omega \nu$ fere ut A, qui $\pi \alpha-$ $\varrho \varepsilon x \times \lambda i \nu \omega \nu$. Recipiendum quod etiam losephus habet $\pi \alpha \rho \varepsilon \gamma-$ $x \lambda i \nu \omega \nu$.

$70,1 \pi \iota \pi \rho \alpha \dot{\sigma} x \varepsilon \sigma \vartheta \alpha \iota] \pi \iota \pi \rho \alpha^{\prime} \sigma x \iota \tau \alpha \iota$, sed $\sigma \vartheta \alpha \iota$ margo r.

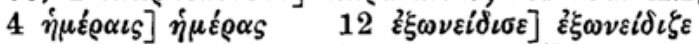

$17 x \alpha \tau \alpha \lambda \varepsilon i \psi \varepsilon \varepsilon v] x \alpha i x \alpha \tau \alpha \lambda \varepsilon i \psi \varepsilon \varepsilon v \quad 23 \delta \varepsilon]$ om. 


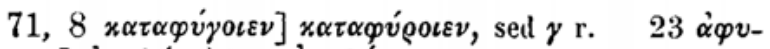
$\left.\lambda \alpha^{\prime} x \tau 0 v \varsigma\right] \alpha^{\prime} \varphi v \lambda_{\alpha}^{\prime} x \tau$ ) i. e. $\alpha \varphi v \lambda \alpha^{\prime} x \tau \omega s$.

$72,1 \tau \tilde{\omega} \nu] x \alpha i \tau \tilde{\omega} \nu \quad 15 \delta \dot{\varepsilon} x \alpha i] \delta \dot{\varepsilon}$.

$73,9 \pi \varepsilon \varrho \varepsilon \sigma \chi \varepsilon \dot{\vartheta} \eta \sigma \alpha \nu] \pi \varepsilon \rho \iota \sigma \chi i \sigma . \vartheta \eta \sigma \alpha \nu \quad 18 \dot{\varepsilon} \mu \pi \alpha-$

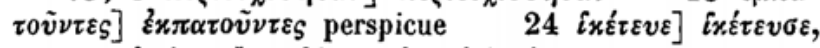
ut $\mathrm{A}$ et plerique Iosephi, quod recipiendum.

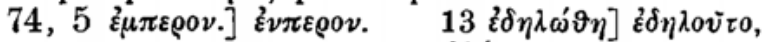

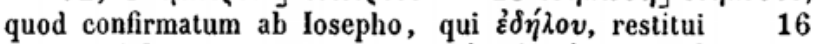

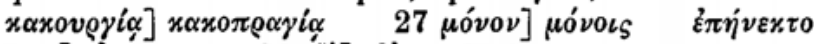
Duc.] $\varepsilon \pi \tilde{\eta} x \tau 0 \quad 29 \quad \delta \dot{\varepsilon}], \delta$ ' ut Wolfius et ego.

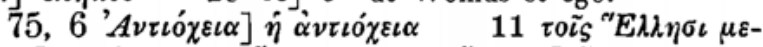

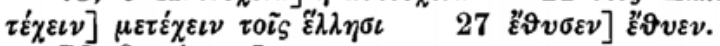

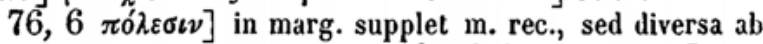

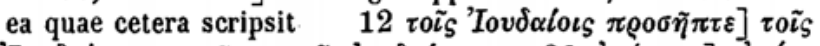

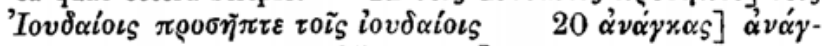

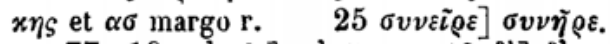

$\left.\left.\left.77,10 \alpha \dot{v} \tau 0 i_{S}\right] \alpha \dot{v} \tau \tilde{\eta}_{S} \quad 12 \delta \dot{\varepsilon}\right] \delta^{\prime} \quad 27 \lambda i \alpha \nu\right]$ supplet $\mathrm{m}$. rec., de qua ad 76,6 .

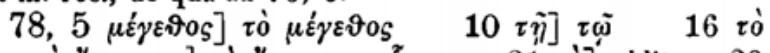

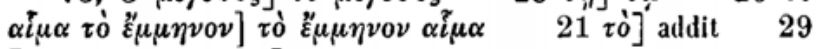
[in marg. corr. 309].

$79,11 \delta \varepsilon \imath \nu \dot{\alpha}]$ addit $\left.\delta \iota \alpha \tau \iota \vartheta \varepsilon i_{S}\right] \delta \iota \alpha \tau \varepsilon \vartheta \varepsilon \iota \varsigma$, sed $\iota$ su-

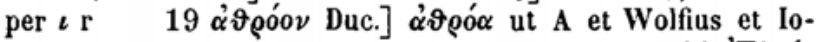
sephus, ut Parisinae vitium sustulisse videar 22 ' $E$ d $\varepsilon \dot{\alpha}$ $\xi \alpha \rho \circ \nu] \varepsilon^{3} \lambda \varepsilon \alpha^{\prime} \zeta \alpha \rho$ ut $\mathrm{A}$, recte, ut antea et postea $24 \dot{i} x \dot{\varepsilon}-$

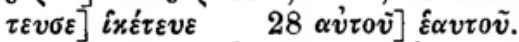

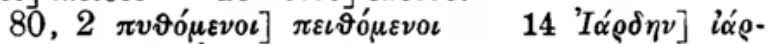
$\left.\delta \omega v \quad 22 \quad \delta_{\varepsilon}^{\prime}\right] \delta^{\prime} \quad 24 \delta \dot{\varepsilon}$ ] in marg. addit manus, de qua ad 76, $\left.630 \delta \iota \alpha \varphi \iota \varepsilon \mu \varepsilon \varepsilon^{\prime} \iota_{\varsigma}\right]$ ita codex ut ed. Ducangii,

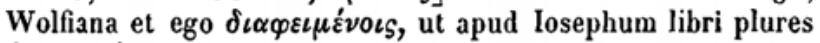
$\delta \iota \alpha \varphi \iota \varepsilon \mu \varepsilon ́ v o \iota$. Quod si est etiam in Parisino, de quo tacetur, Zonarae est restituendum 31 'E $\left.\mu_{\alpha o} \tilde{v}_{S}\right] \dot{\alpha} \mu \alpha o \tilde{v}_{s}$.

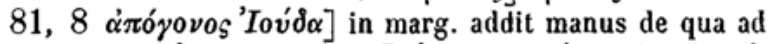

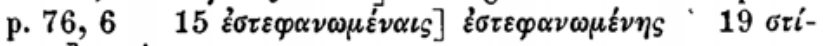
xovs] oroixovs.

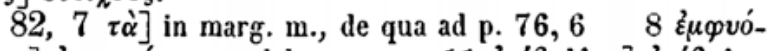

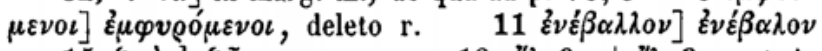

$15 \xi \omega o ̀ s] \xi \tilde{o} \nu \nu$ eraso o $\left.19 \varepsilon^{\prime \prime} \lambda \alpha \vartheta \varepsilon \nu\right\rfloor \varepsilon ้ \lambda \alpha \vartheta o \nu$ ut A, 
quod recipiendum foret, nisi $\varepsilon^{\prime \prime} \lambda \boldsymbol{\vartheta} \varepsilon$ haberet Iosephus $7,9,1$,

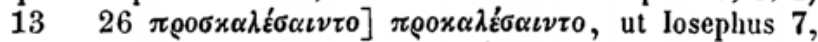

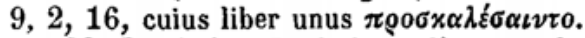

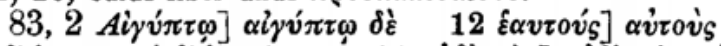

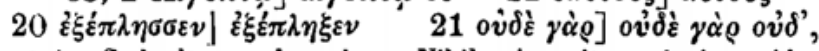
ut A. Sed alterum losephus. Nihilominus ita scripsisse videtur Zonaras, ut vol. 2, p. 269, B.

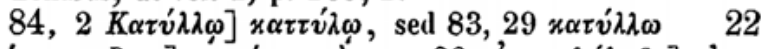

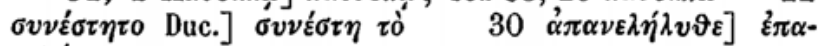
$\nu \varepsilon \lambda \eta^{\prime} \lambda v \vartheta \varepsilon$.

$85,4, \mu \dot{\eta}]$ om. et addit margo r. 6 inscr. col. rubr.

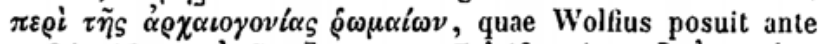

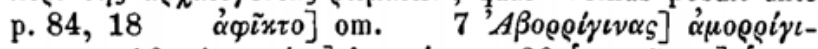

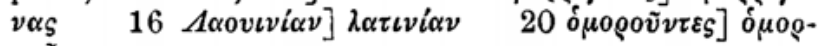

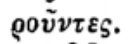

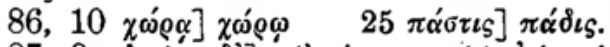
$\lambda i v o v$

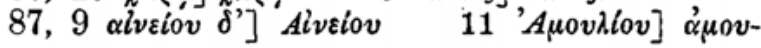

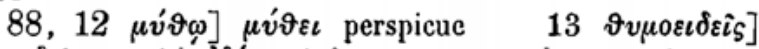

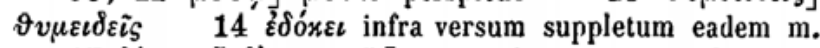

$\left.17 \delta \varepsilon \dot{\varepsilon} \pi 0 \tau \varepsilon] \delta \dot{\varepsilon} \quad \tau 0 \tilde{\iota}_{S}\right] \tau \eta_{\zeta}$, sed $o \iota$ s. v. r. $26 \vartheta \alpha \rho-$

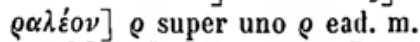

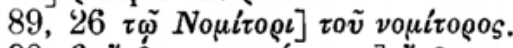

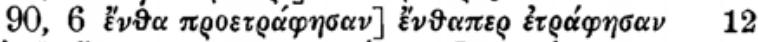

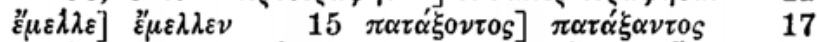

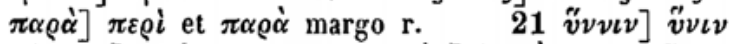

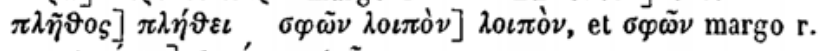

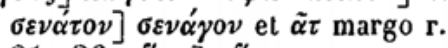

$91,26$ ov' $\tau \omega]$ ovं $\tau \omega s$, recte.

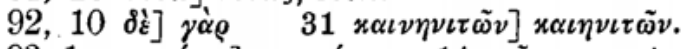

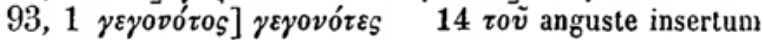
eaclem $\mathrm{m}$.

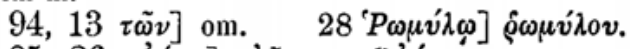

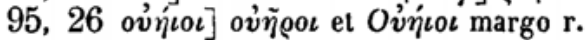

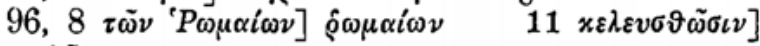
$\varkappa \lambda \varepsilon v \sigma \vartheta \tilde{\eta} \nu \alpha t$.

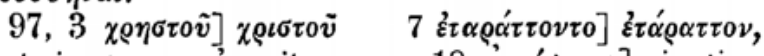
et asteriscus super $\bar{\varepsilon}$ positus r. $18 \dot{\alpha} \mu \varepsilon i \psi o \iota \tau 0]$ sic etiam 
hic pro $\alpha \mu \varepsilon i \psi \alpha \iota \tau o$, quod scripsi $\vartheta_{\left.\varepsilon 0 i_{S}\right]} \vartheta \varepsilon \dot{\varepsilon}_{S}$ et $0 \tilde{\imath}$ margo r. $\left.20 \delta^{\prime}\right] \delta \dot{\varepsilon}$.

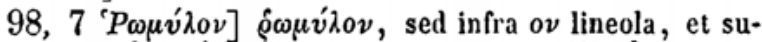
pra $v \quad 8 \dot{\alpha} \nu \varepsilon x \tau \dot{\omega} \nu] x$ s. v. ead. m. $\quad 15 \delta \dot{\varepsilon}] \delta^{\prime}$.

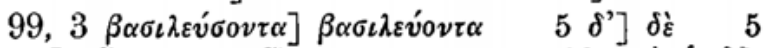

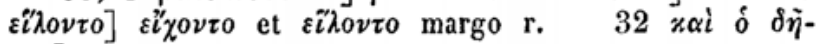
$\mu \mathrm{s}] \mathrm{om}$.

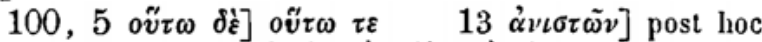

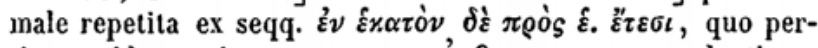
tinere videtur signum $r$. super $\alpha \nu \vartheta \rho$. versu praecedenti po-

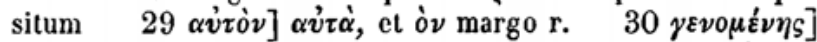
$\gamma \iota \nu 0 \mu \varepsilon \dot{v} \eta S$ corr. in prima r., ut $\gamma \varepsilon$ fuerit pr. $\pi 0 \lambda \varepsilon \dot{\mu} \iota \iota \iota]$ supplet margo al. m., diversa a ceteris in marg.

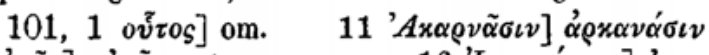
$\left.14 \varepsilon^{\prime} \tau \tilde{\omega} \nu\right] \alpha i \tau \tilde{\omega} \nu$, et $\varepsilon$ s. v. r. 16 'I $\left.\alpha \nu 0 v \alpha \varrho \iota \nu \nu\right] l \alpha \nu \nu 0 v \alpha$ -

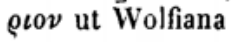
$18 \sigma v \nu \varepsilon \vartheta i \sigma \alpha \nu \tau o s]$ 6 postremum s. v. r.

$30 \vartheta v \gamma \alpha \tau \rho \iota \delta 0 \tilde{v} s]$ จ $v \gamma \alpha \tau \varrho \iota \delta \dot{\eta} S$ ut A. Eadem forma in utroque libro mox p. 103, 24, et in hoc vol.1, p. 636, B, in A vol. 2, p. 51, B, ubi $\vartheta v \gamma \alpha \tau \varrho \iota \delta \eta \dot{v}$ praebet pro $\vartheta v \gamma \alpha \tau \varrho \iota \delta o \tilde{v} \nu$, et in omnibus ib. p. 161, C, ubi $\boldsymbol{\vartheta} v \gamma \alpha \tau \rho \iota \delta \eta^{\prime}$. Et quanquam

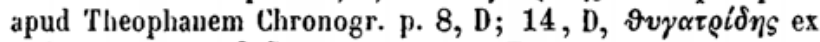
cod. in $\vartheta v \gamma \alpha \tau \rho \iota \delta o \tilde{v}_{S}$ est mutatum, Zonaram tamen utraque usum esse forma ostendunt loci citati. Nam ut $\lambda \varepsilon o v \tau \iota \delta \eta_{S}$ dixerunt recentiores pariter atque $\lambda \varepsilon 0 \nu \tau \iota \delta \varepsilon v_{\varsigma}$, de quo v. Thes. Stephani, ita alia multa eadem dixerunt forma in $\delta \eta_{S}^{\prime}$, quae post Eustathium 0d. p. 1821, 35, memoravit Plilemo ad Stephanum v. $\Lambda \alpha \gamma i \delta \eta s$ citatus. Quam recentiorum consuetudinem ignorans Valckenarius, ut dictum ibidem, et haec omnia ficta opinatus ex formis in $\varepsilon v_{\varsigma}$ corrigebat quae sunt quidem aliena a veteribus, sed recentioribus relinquenda, nerlum ut ad Atticas formas pluralis in $\tilde{\eta}_{S}$ sint revocanda, quae nonnullorum fuit opinio. Confusionem in his formis produnt etiam

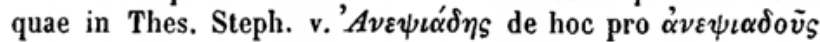
illato annotavi et magis etiam mirum apud Annam Comn. p. $168, \mathrm{D}$, oi $\dot{\alpha} \nu \varepsilon \psi \iota \alpha \delta \varepsilon i_{S}$, et p. $265, \mathrm{C}, \tau 0 \dot{v}_{\zeta} \alpha \dot{v} \tau o \dot{v} \alpha v \varepsilon-$ $\psi \iota \alpha \delta \varepsilon \tilde{L}_{s}$.

$102,1 \kappa \alpha \tau \dot{\alpha}]$ ] $x \alpha i$, et $x \alpha \tau \dot{\alpha}$ margo r. $15 \pi \alpha \rho \varepsilon \chi \omega \dot{\rho \varepsilon \iota]}$ 
$\pi \alpha \rho \varepsilon \chi \omega ́ \rho \nu v \quad 18 \delta \dot{\varepsilon}]$ om. $\left.29 \delta v^{\prime} \omega\right] \delta v_{o}$ ut Pinderus tacito.

103, $\left.24 \vartheta v \gamma \alpha \tau \varrho \iota \delta o \tilde{v}_{\varsigma}\right] \vartheta v \gamma \alpha \tau \varrho \iota \tilde{\eta}_{S}$ ut A, recte. V. ad p. 101,3027 rubro col. superscr.: $\beta \alpha \sigma \iota \lambda \varepsilon i \alpha \mu \alpha \varrho x i o v$

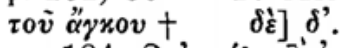

$\left.104,2 \dot{\alpha} \gamma x v^{\prime} \lambda \eta\right]^{\prime} \dot{\alpha} \gamma x v^{\prime} \alpha \nu$, compendio scripto $\alpha v$ ut in

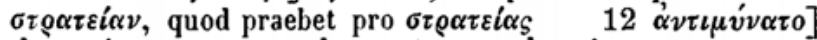

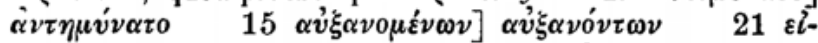

$$
\pi
$$

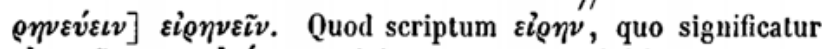

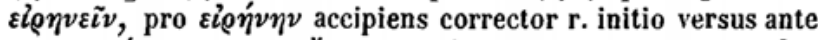
$\pi \alpha \varrho \varepsilon \sigma x \varepsilon v \alpha \sigma \varepsilon \nu$ addidit $\alpha$ $\gamma \varepsilon \iota \nu \quad 26$ rubro coll. superscr. $\beta \alpha-$

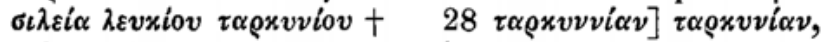
sed mox ipse quoque $\tau \alpha \rho x v \nu v \eta \sigma i \omega v$.

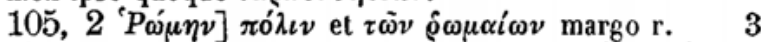

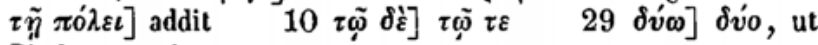
Pinderus tacito.

106, $7 \delta \dot{\varepsilon}]$ om. C $\quad 9 \tau \iota v \iota]$ s. v. ead. m. $\delta \dot{\varepsilon}] \gamma \dot{\alpha} \rho$

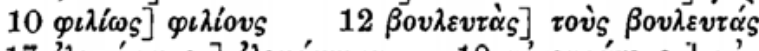

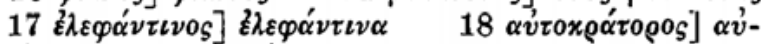

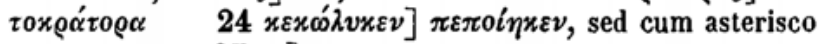
super $\pi$ r., $27 \tau \varepsilon]$ om.

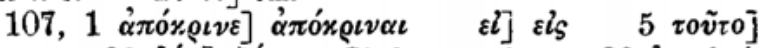

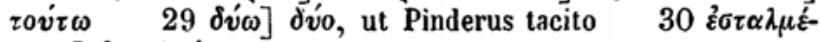
vovऽ] $\varepsilon \sigma \tau \alpha \lambda \mu \varepsilon \dot{v} \alpha \iota_{\varsigma}$, sed $o v$ s. v. r.

$\left.\left.108,7 \tau \dot{\eta} v \delta \dot{\varepsilon} \tau \tilde{\eta} S^{2} P \omega \mu_{\mu}\right\rangle\right]$ his rubro col. superscr.:

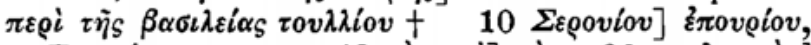

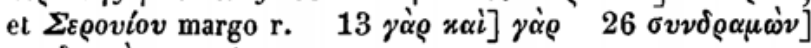
$\sigma v \nu \delta \varrho \alpha \mu o \dot{v}$ perspicue.

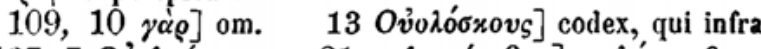

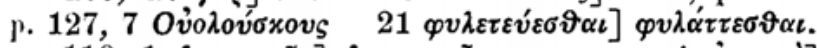

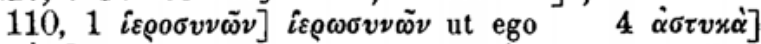
$\dot{\alpha} \sigma \tau \iota x \dot{\alpha}$ [Revocandum autem post hoc $\alpha \alpha i$ a Pindero sollici-

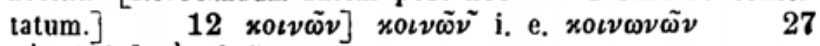
$\tau \dot{\eta} v \beta o \tilde{v} v] \tau \dot{\nu} v \beta \circ \tilde{v} \nu$.

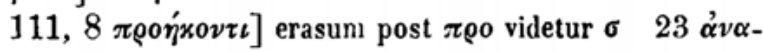

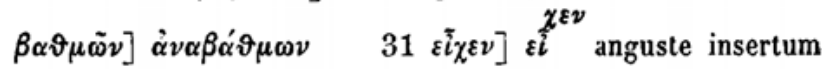


super alia littera et $\varepsilon$ sequentis $\dot{\varepsilon} \pi \dot{\eta} \lambda \alpha \sigma \varepsilon \nu$, etsi non fuit $\dot{\alpha} \pi \dot{\eta}$ $\lambda \alpha \sigma \varepsilon \nu$.

112, 1 ov๊ $v]$ om. $\quad 8 \tau v \rho \alpha v v i \sigma \omega \nu] \tau v \rho \alpha \nu v \eta^{\prime} \sigma \omega v$.

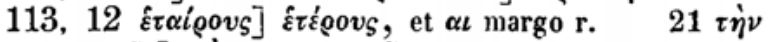
$\tau \tilde{\omega} \nu \pi 0 \lambda \iota \tau \iota x \tilde{\omega} \nu] \tau \dot{\alpha} \tau \tilde{\omega} \nu \pi 0 \lambda \iota \tau \tilde{\omega} v$, sed asteriscum margo r.

$32 \Gamma \alpha 0 v i v \omega \nu] \gamma \alpha v v i \omega v$.

114, $14 \sigma v \mu \beta$ ov $\lambda \dot{\eta} v] \beta 0 v \lambda \grave{\eta} v$ ut A, quod verum videtur.

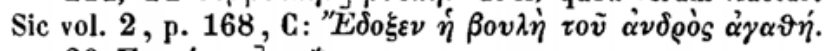

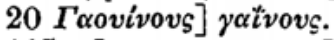

115, [in inscr. corr. Xl] $\left.10 \delta v^{\prime} \omega\right] \delta$ vio hic et 12 et 18

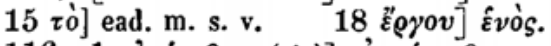

$116,1 \dot{\alpha} \nu \varepsilon \dot{\rho} \varepsilon \vartheta \alpha \iota(\mathrm{sic})] \dot{\alpha} \nu \alpha i \varrho \varepsilon \sigma \vartheta \alpha \iota \quad 8 \pi \alpha \lambda \alpha \tau i \omega]$

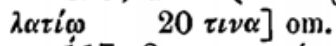

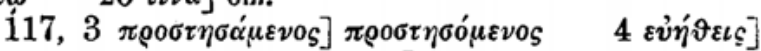

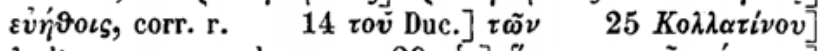

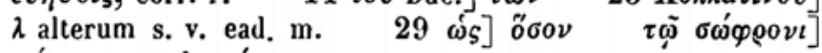

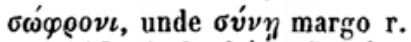

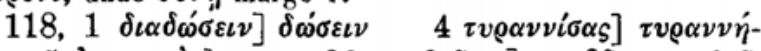

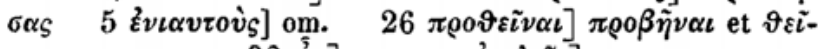
$\nu \alpha \iota$ margo r. $\left.\quad 30 \eta^{5} \nu\right]$ om. $\left.\quad \alpha \nu \varepsilon \lambda \varepsilon \tilde{\nu} \nu\right]$ om.

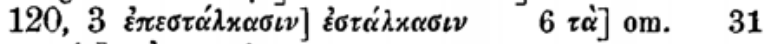

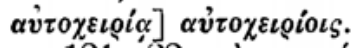

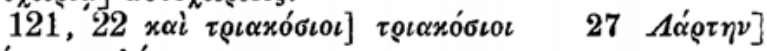
$K \lambda \dot{\alpha} \rho \alpha \nu$, et $\lambda \alpha \dot{\alpha} \tau \eta \nu$ margo r.

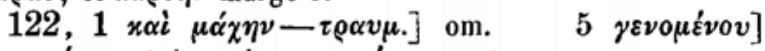

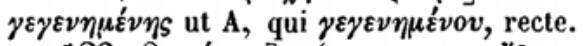

123, $\left.8 \mu \alpha^{\prime} \chi \alpha \iota s\right] \mu \alpha \chi^{\varepsilon} \varepsilon \iota$ propter $\varepsilon$ है $\nu \varepsilon \sigma \iota$, et $\alpha \iota \sigma$ margo r.

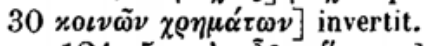

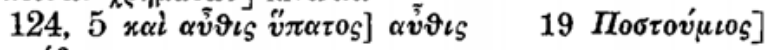

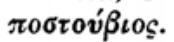

125, $11 \dot{\alpha} \gamma \alpha \gamma \dot{\omega} \nu] \dot{\alpha} v \alpha \gamma \alpha \gamma \dot{\omega} v$

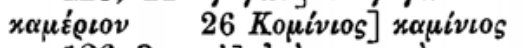

$23 K \alpha \mu \varepsilon \rho i v o v$ Duc.] $30 \delta 0 v ́ \lambda \omega \nu]$ om.

126. $3 x \alpha \tau \dot{\alpha}] \dot{\alpha} \pi \dot{0}$, et $\kappa \alpha \tau \dot{\alpha}$ margo r. $13 \hat{\eta} v \mu \dot{\varepsilon} v$ Duc.]

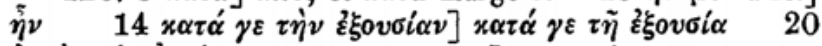
$\dot{\alpha} \lambda \lambda \dot{\alpha} x \alpha i] \dot{\alpha} \lambda \lambda \dot{\alpha} \quad 24 \pi \alpha \rho \varepsilon \tau \varepsilon i v \alpha \tau 0] \pi \alpha \varrho \varepsilon \tau \varepsilon i v \varepsilon \tau o$.

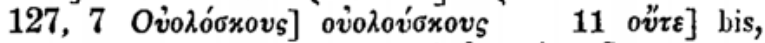

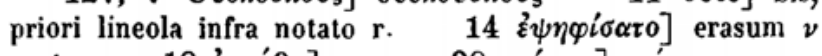

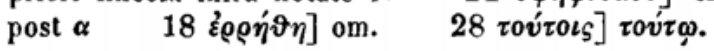


128, 6 हैं $\iota]$ om. $\left.\quad 8 \mu \eta \delta^{\delta} v^{\prime}\right] \mu \eta^{\prime} \varepsilon \varepsilon$ fere ut A, qui $\mu \eta^{\prime} \iota$, ut $\left.129,1611 \delta v \sigma \mu \alpha \chi \omega^{\prime} \alpha \tau \sigma \nu\right] \delta v \sigma \mu \alpha \chi o ́ \tau \alpha \tau o \nu$.

$129,15 \varepsilon \dot{\varepsilon} \pi i \sigma \alpha \nu]$ om. et $0 \nu \tau \varepsilon \sigma$ margo r. $200 \hat{i}] \alpha \hat{i}$, sed $o$ in $o \iota$ mutato r.

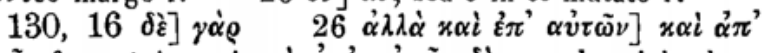

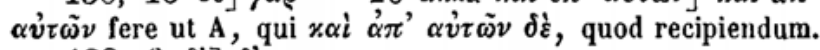

$132,6 \quad \delta \dot{\varepsilon}] \delta^{\prime}$.

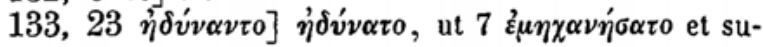
pra v. eadem $\mathrm{m}$. scriptum $\nu$.

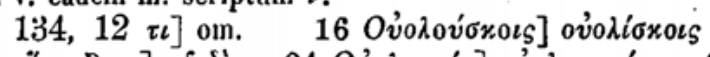

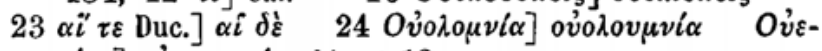

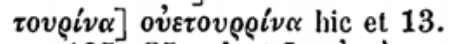

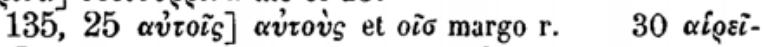
$\sigma \vartheta \alpha \iota]$ solum $\sigma \vartheta \alpha \iota$ initio versus, unde $\alpha i \rho \varepsilon i \hat{~ a d ~ m a r g . ~ p r a e c e-~}$ dentis post $\varepsilon \dot{v} \pi \alpha \tau \rho \iota \delta \tilde{\nu} \nu \mathrm{r}$. $\quad x \alpha \tau \varepsilon \iota \rho \gamma \alpha \dot{\alpha} \alpha \nu \tau \nu] \nu \mathrm{s}$. v. ead. m.

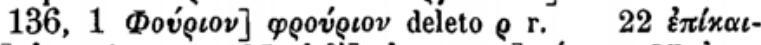

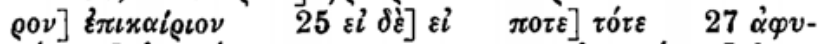

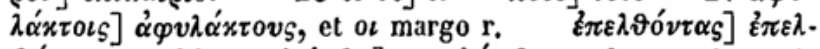

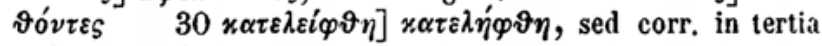
eadem, ut videtur, $\mathrm{m}$.

$\left.137,4 \delta^{\prime}\right] \delta \dot{\varepsilon}$.

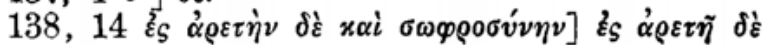

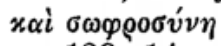

31 Ko@ovivov] no@ov́ıvov.

139, $\left.14 \pi \varepsilon \pi \alpha^{\prime} \varphi \varphi \alpha \iota\right] \pi \varepsilon \pi o^{\mu}$ in fine versus $\left.30 \delta^{\prime}\right]$ $\delta \dot{\varepsilon}$ ut Wolfius.

140, 7 'P

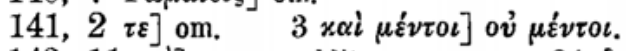

143, $11 \mu \eta]$ om. et addit margo r. $21 \delta \iota x \tau \alpha$. $\left.2 \rho \alpha_{s}\right]$

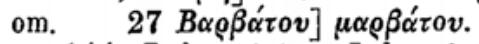

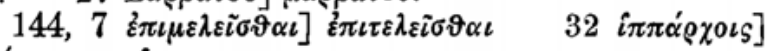
vं $\alpha^{\circ} \varrho \chi 0 \iota s$, et $i \pi \pi$. margo $\mathrm{r}$.

$145,4 \ddot{o} \nu] \mathrm{om}$. et addit margo $\mathrm{r}$. $6 \sigma \nu \mu \pi \alpha \dot{\alpha} \nu \omega \nu]$ $\sigma v \mu \beta \alpha^{\alpha} \nu \tau \omega \nu$ et $\pi$ margo r. $\left.19 \mu \dot{\varepsilon} \nu\right]$ om. $20 \pi \rho 0 \sigma \omega-$

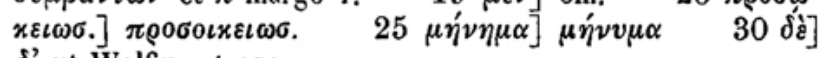
$\delta$ ' ut Wolfius et ego.

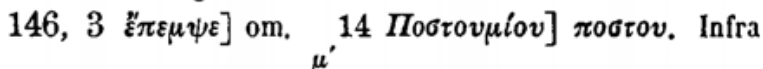
hoc nomen scribitur $\pi 0 \sigma$ tov . 


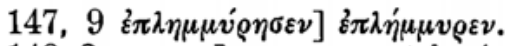

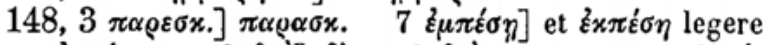
licet et $\left.\xi_{\mu}^{\prime} \mu \dot{\varepsilon} \sigma \eta \quad 8 \delta \iota \dot{\alpha}\right] \delta \dot{\varepsilon}$, sed $\delta \iota \dot{\alpha}$ margor. $13 \nu \dot{\varepsilon}$ -

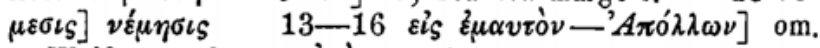
ut Wolfius, sed ante $ə \dot{v} \chi \dot{\eta} \nu$ asteriscus $\mathrm{r}$.

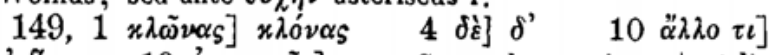

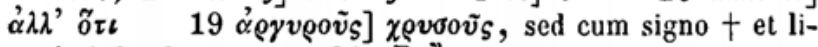
neola infra ducta $\mathbf{r}$. $20 \varepsilon i]$,

$\left.150,1 \varepsilon \delta \alpha \pi \alpha^{\alpha} \nu \omega \nu\right] \delta \alpha \pi \alpha^{\prime} \nu \omega \nu$ et $\varepsilon \iota$ margo r.

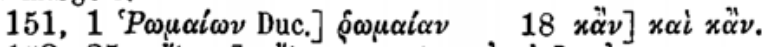

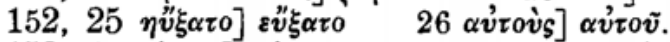

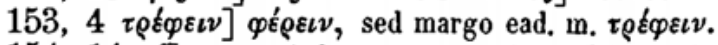

$154,14$ oi $]$ om. et infra versum paginae ultimum inser-

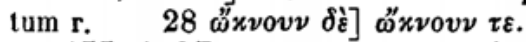

$155,1 \delta \varepsilon]$ fere ut $\mathrm{A}$, qui $\tau \varepsilon x \alpha \dot{i}$, et p. $357, \mathrm{C}$ pro ö́ $\tau \omega$

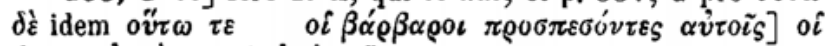
$\beta$. $\pi$. $\left.\alpha v_{\tau o v} \quad 4 \dot{\eta} \mu \dot{v}^{\prime} \rho \alpha \varsigma\right]$ in fine versus ascriptum in marg. ead. $\mathrm{m}$., quod in A transpositum post $\varepsilon \pi \iota \chi \varepsilon \iota \varrho 0 \tilde{v} \nu \tau \varepsilon$.

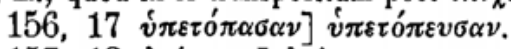

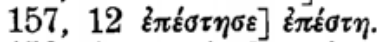

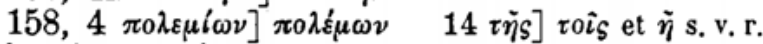

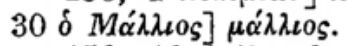

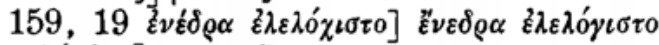
$\left.x \alpha \tau \alpha x \lambda \alpha^{\prime} \sigma \vartheta \alpha \iota\right] x \alpha \tau \alpha x \lambda \tilde{\alpha} \sigma \vartheta \alpha \iota$ ut ego.

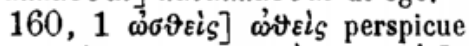

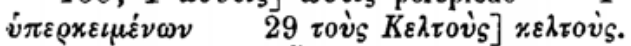

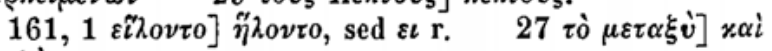
$\mu \varepsilon \tau \alpha \xi \dot{v}$.

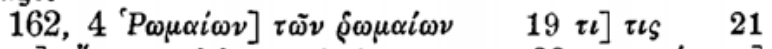

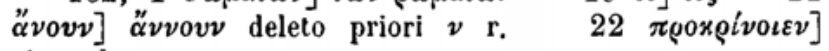
sic codex.

163, $\left.11 \varepsilon^{2} v \eta^{\prime} \lambda \alpha \tau 0\right] \varepsilon^{2} v \eta^{\prime} \lambda \lambda \alpha \tau o$.

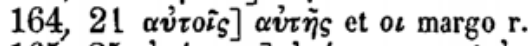

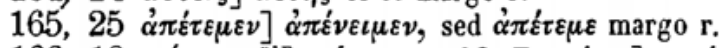

166, $\left.\left.18 \pi \alpha^{\prime} \nu \tau \omega_{S} \delta_{\dot{\varepsilon}}\right] \pi \alpha^{\prime} \nu \tau \omega \varsigma \quad 32 \Sigma \alpha v v i \tau \alpha_{S}\right] \sigma \alpha v i \tau \alpha_{S}$.

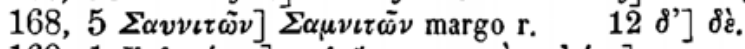

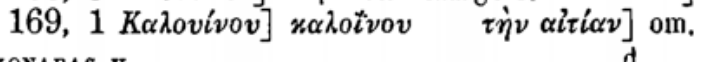
zONARAS $v$. 


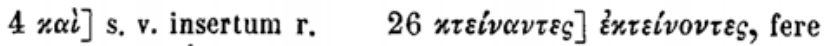
ut $\mathrm{A}$, qui $\varkappa \tau \varepsilon i \nu 0 \nu \tau \varepsilon s$.

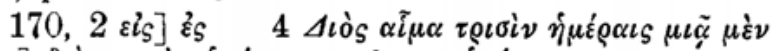

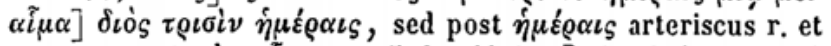

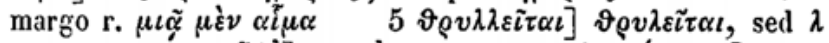

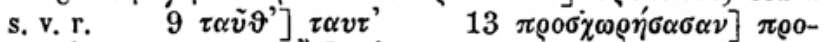
$\chi \omega \varrho \eta ́ \sigma \alpha \sigma \alpha \nu 15 x \alpha \alpha^{\prime \prime} x_{\alpha \alpha} \nu$, sed cum asterico r., etsi nihil est in marg. $\alpha \dot{v} \tau o v \dot{s}$ ] $\alpha \dot{v} \tau o \tilde{v}$, sed cum asterisco r. ante $\delta \iota^{\prime}$.

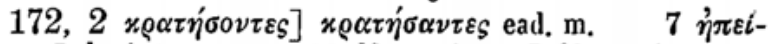

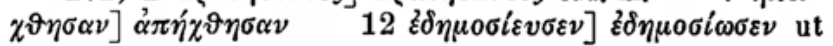
$A$, et $\varepsilon v$ margo r.

$\left.173,15 \pi \varrho \circ \beta \alpha \lambda_{0} \mu \varepsilon v \sigma_{\varsigma}\right]$ alterum $\lambda$ priori additurus librarius ex eo fecit o $27 \pi \rho 0 \sigma \tilde{\eta} \pi \tau \varepsilon] \pi \varrho 0 \sigma \alpha ́ \pi \tau \omega \nu$.

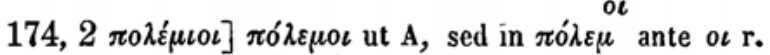
addit $\iota \quad 10$ Aovxiov] $x \alpha i$ addit, quod lineola infra notatum r. 11 ó $\tau \iota$ Duc.] "ó $\pi \eta \quad 18$ $\left.\delta \dot{\varepsilon} \tau \alpha \tilde{v} \vartheta^{\prime}\right] \tau \alpha \tilde{v} \vartheta^{\prime}$.

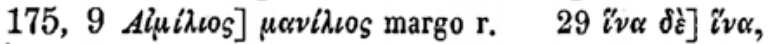
et $\delta \grave{\varepsilon}$ margo $\mathrm{r}$.

$176,18 \pi \varrho 0 \sigma x \alpha \rho \tau \varepsilon \varrho \varepsilon \tilde{\nu}]$ om. et $\dagger$ ante ov่ $\chi$ s. v. r.

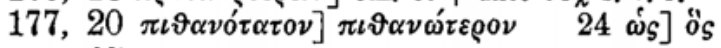
$\delta \dot{\varepsilon}$ ] om., aldit margo $\mathrm{r}$.

$\left.\left.178,2 \sigma v \mu \mu \alpha \chi i \delta \alpha_{S}\right] \sigma v \mu \mu \alpha \chi i \alpha \alpha_{S} \quad 6 \pi \varepsilon \rho i\right] \tau \varepsilon$, et $\pi \varepsilon \rho i$

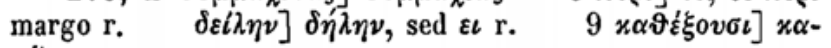

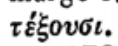

$\left.179,2 x \alpha \vartheta \iota \sigma \tau \alpha_{s}\right] x \alpha \vartheta i \sigma \alpha s$, ut videtur, pr., correctus ead. m. $9 \dot{\eta} \pi \varepsilon i$ $9 \varepsilon 0] \dot{\eta} \pi \dot{\eta} \gamma \varepsilon \tau o$, secunda eadem manu correcta, primo vero $\eta$ perspicue sic scripto $\oint \varepsilon \tilde{v} \mu \alpha \tau \sigma \tilde{v}]$ om. C., unde corrector et $\nu$ addidit ad $\tau \dot{\circ}$ et in $\pi 0 \tau \alpha \mu o \tilde{v}$ lineola notavit $0 \tilde{v} \quad 17-18$ हैं $\pi \varepsilon v \sigma \varepsilon-\varepsilon x \pi \varepsilon \pi \lambda \eta \gamma \mu \varepsilon \dot{\varepsilon} \nu \omega \nu]$ om.

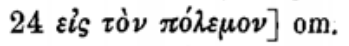

$180,4 \tau \dot{\eta} \nu] \tau 0 \tilde{i} s$, lineola notatum r. $\left.11 \alpha \dot{\alpha} \pi \mu v_{v} \nu \omega \nu\right]$ ह่ $\pi \alpha \mu v \dot{v} \omega \nu 17 \gamma v \mu \nu \tilde{\eta}] \sigma \varepsilon \mu \nu \tilde{\eta}$, et $\gamma \nu \mu \nu \tilde{\eta}$ margo $\mathrm{r}$.

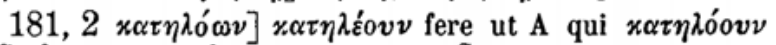
$\left.3 \varepsilon i_{S} \dot{v} \pi \varepsilon \lambda \varepsilon i \varphi \vartheta \eta\right] \dot{v} \pi \varepsilon \lambda \varepsilon i \varphi \vartheta \eta$, unde $\varepsilon \tilde{i}_{S}$ addit margo r. $14 \varepsilon^{2} \chi \varepsilon\llcorner\varrho \omega \sigma \alpha ́ \mu \eta \nu]$ om., unde post $\pi \tilde{\alpha} \sigma \alpha \nu$ supra v. signum $+\mathrm{r}$. 
$\left.19 \mu \varepsilon^{\prime} \lambda \lambda \eta \sigma \iota \nu\right] \mu \varepsilon^{\prime} \lambda \eta \iota \nu$, et $\lambda$ s. v. r. $\left.22 \mu \varepsilon \tau \varepsilon \pi \varepsilon^{\prime} \mu \psi \alpha \nu \tau 0\right]$ $\nu$ s. v. ead. m. $29 \delta^{\prime}$ om. Duc.] addit.

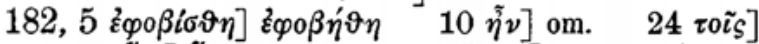

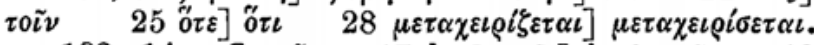

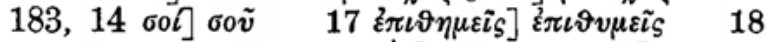
$\gamma \varepsilon$ ] $\sigma \varepsilon$, et $\gamma \varepsilon$ margo r. $\left.\left.19 \varphi \dot{\eta}_{S}\right] \varphi \tilde{\eta}_{S} \quad 21 \tau \iota\right]$ anguste insertum ead. $m$.

184, $16 \dot{v} \mu \tilde{\omega} \nu] \dot{\eta} \mu \tilde{\omega} \nu$, corr. r. $\left.22 \delta^{\prime}\right] \delta \dot{\varepsilon}$ ut Wolfius et ego et, opinor, A.

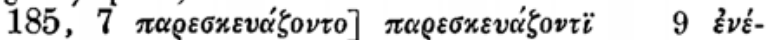
$\beta \alpha \lambda \lambda \varepsilon]$ ह่vह́ं $\beta \alpha \lambda \varepsilon \quad 13 \gamma \dot{\alpha} \varrho]$ om.

186, $\left.17 \pi \rho \circ \varepsilon \chi 0 v ́ \sigma \alpha_{S}\right] \pi \varrho \circ \sigma \varepsilon \chi 0 v ́ \alpha_{S}$.

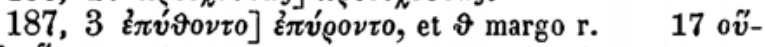

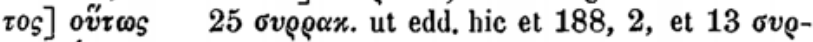

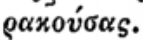

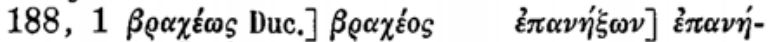

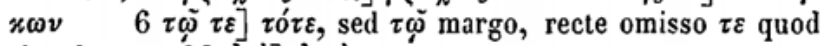
abundat 30 ह $\varepsilon i]$ हं $\pi \varepsilon i$.

189, 4 ov $v]$ om. $\left.\quad 9 \lambda \varepsilon^{\prime} \gamma o v \tau \alpha\right] \lambda o^{\prime} \sigma \nu$, corr. ead. m.,

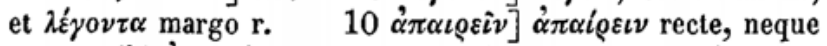
opus scribi $\alpha \pi \alpha \varrho \varepsilon \tilde{\nu} \nu$, ut ex accentu illo colligendum videbatur

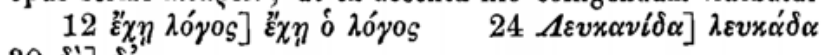
$30 \quad \delta \dot{\varepsilon}] \delta^{\prime \prime}$.

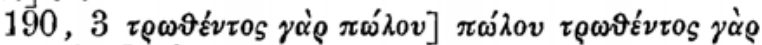

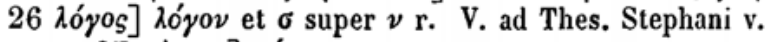

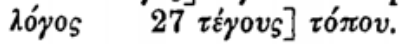

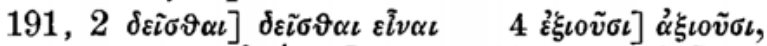
sed $\varepsilon$ s. v. r. 10 ह่ $\pi \eta \dot{\varepsilon} \varepsilon \sigma \alpha \nu]$ om. $22 K \alpha \rho \circ v\llcorner\lambda[o v] x \alpha-$ $\lambda$

gä̈ov cum ductu ov significante.

192, 7 ó $\tau]$ ó $\tau \varepsilon$, sed $\iota \mathrm{r} . \quad 11 \mu \alpha \mu \varepsilon \rho \tau i \nu 0 v \varsigma] \mu \alpha \rho \mu \varepsilon \nu-$

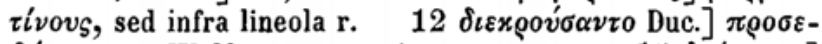

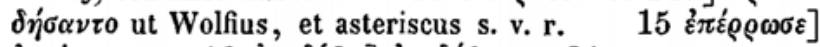

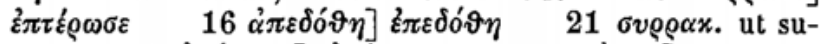

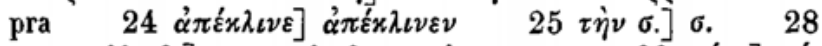
$x \varrho v \sigma \tau \alpha \lambda \lambda \omega \vartheta$.] $x \varrho v \sigma \tau \alpha \lambda \omega \vartheta$, et $\lambda$ s. v. r. $\left.30 \pi \alpha_{\varsigma}\right] \pi \sigma^{-}$ $\lambda$ $\lambda \varepsilon \omega \varsigma$ ( $\left.\pi o^{\prime}\right)$, et $\pi \operatorname{ló}_{\varsigma}$ margo $\mathrm{r}$. 


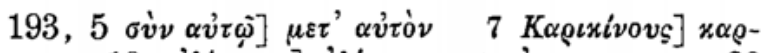
xivovs $18 \varepsilon \dot{v} \lambda i \mu \varepsilon \nu 0 \nu] \dot{\alpha} \lambda i \mu \varepsilon v o v$, et $\varepsilon \dot{v}$ margo r. $\quad 20$

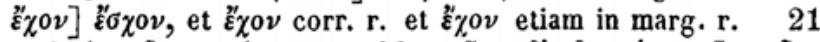

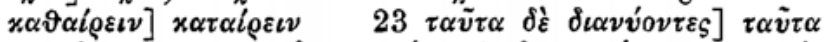

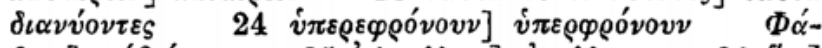

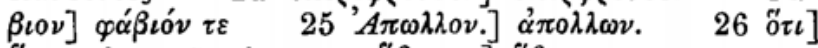

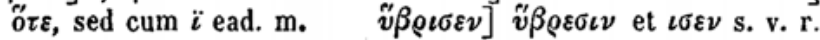
30 oi] oi.

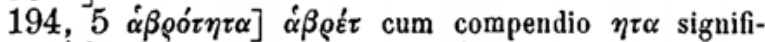
cante, et $\alpha \beta \rho o ́ \tau \eta \tau \tau$ margo r. 6 roi s] $\tau \alpha i_{\varsigma}$, sed o s. v. r.

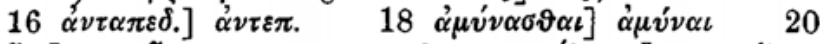

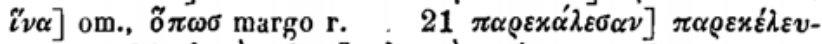

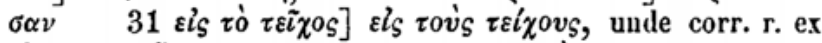

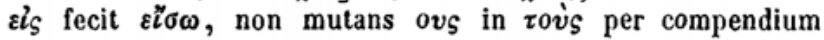
scripto.

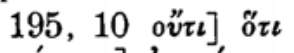

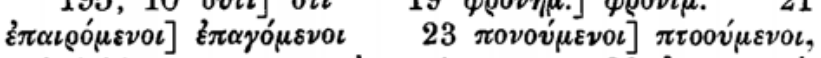
unde initio versus ante $x \alpha i$ asteriscus r. $30 \delta\llcorner\alpha \nu \varepsilon \nu 0 \eta \mu \varepsilon ́-$

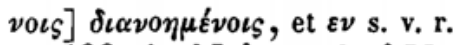

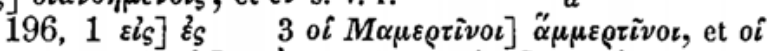

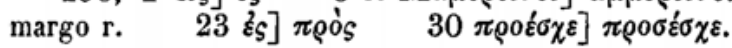

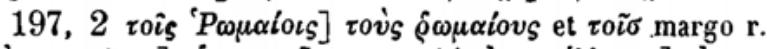

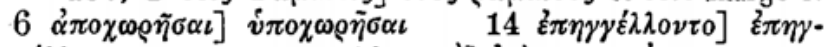
$\gamma \varepsilon \dot{\lambda} \lambda \varepsilon \tau 0$, et $0 \nu$ s. v. r. $16 \pi \alpha \rho \dot{\alpha}]$ ż $\pi i$, et $\pi \alpha \rho \dot{\alpha}$ margo r.

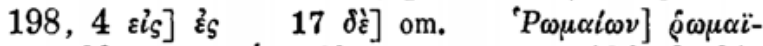

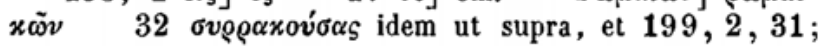
$200,11$.

$199,6 \mu \nu \nu \omega \vartheta \varepsilon \hat{\sigma} \sigma \iota v] \mu 0 \nu 0 \vartheta \varepsilon \tilde{\imath} \sigma \iota v$, et $\omega$ s. v. r. $8 \chi \varepsilon \rho-$

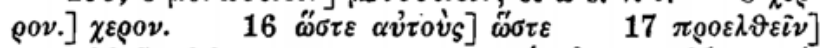

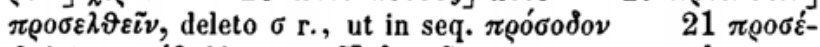
$\beta \alpha \lambda \varepsilon] \pi \varrho \circ \sigma \varepsilon \beta \alpha \lambda \lambda \varepsilon \quad \tau \tilde{\varepsilon}] \varepsilon \varepsilon_{\varepsilon} \nu \tau \tilde{\omega}$, sed lineola infra $\varepsilon v \mathrm{r}$.

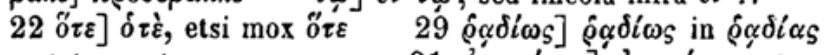
mutatum, et $\omega$ margo r. $\quad 31 \stackrel{\alpha}{\alpha} \pi \alpha \nu \varepsilon \sigma \tau \eta]^{\prime} \varepsilon \alpha \nu \varepsilon \dot{\varepsilon} \sigma \eta \eta$, et $\alpha$ margo $r$.

$200,6 \stackrel{\alpha}{\alpha} \mu \varphi \omega] \stackrel{\not}{\alpha} \mu \varphi \omega x \alpha i$, cum lin. infra $x \alpha i$ r. $\quad 8 \delta$

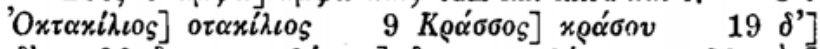

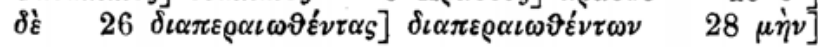




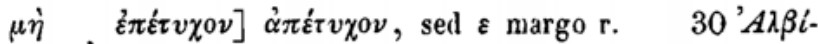
vov] $\alpha \lambda \alpha i v o v$.

201, 19 ö om. Duc.] addit , $4 \alpha \dot{v} \hat{\omega}]$ om. $\sigma v \mu \mu \alpha-$ $\left.\left.\left.\chi \eta^{\prime} \sigma 0 \nu \tau \alpha\right] \sigma v \mu \mu \alpha \chi \eta^{\prime} \sigma \alpha \nu \tau \alpha \quad 16 \dot{\alpha} x \mu \dot{\eta} \nu\right] \dot{\alpha} x \mu \dot{\eta} \quad 17 \alpha \dot{v}{ }^{\prime} \hat{i}_{S}\right]$ om. $19 \varepsilon \pi \varepsilon \chi \varepsilon i \varrho \eta \sigma \varepsilon] \varepsilon \pi \iota \chi \varepsilon i \varrho \eta \sigma \varepsilon \quad 24 \dot{\alpha} \delta \varepsilon \tilde{\omega} \varsigma] \delta \varepsilon \delta \iota \omega_{\varsigma}$, alterum margo $r$.

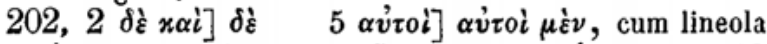

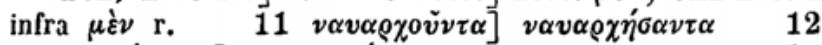

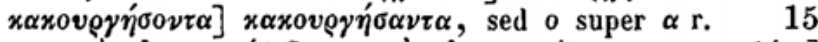

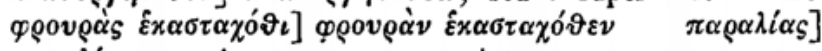
$\pi \alpha \varrho \alpha \lambda i o v$ pers picue, ov super $\iota$ scripto.

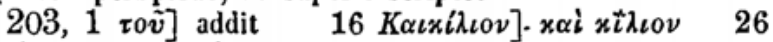
$\sigma v \nu \alpha \dot{\pi} \pi \tau 0 \iota v \tau 0] \sigma v \nu \alpha \dot{\pi} \pi \tau \iota \nu \tau \tau$.

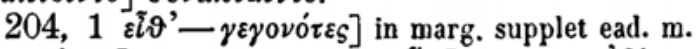

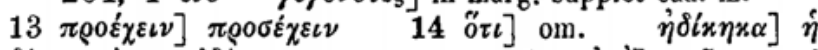
$\delta i x \eta x \alpha i$, et $\eta \delta i x \eta x \alpha$ margo r. $15 \tau \alpha v i \alpha]$, $\tau \alpha \tilde{v} \tau \alpha$, sed

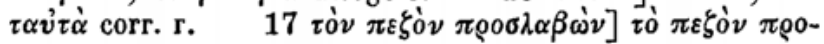

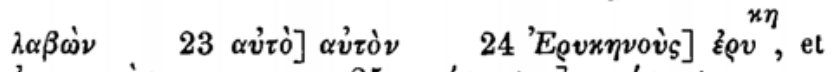

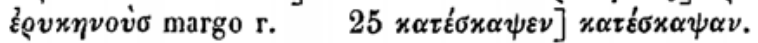

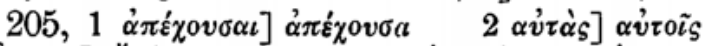

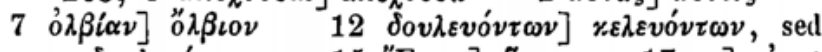

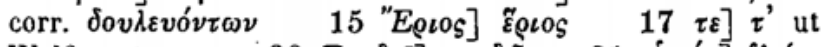

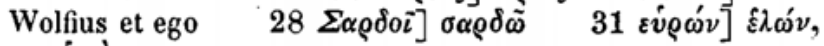
et $\varepsilon \dot{v} \varrho \omega \dot{\nu}$ margo r.

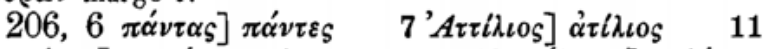

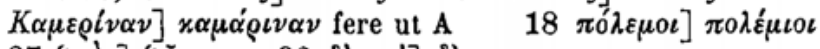

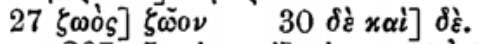

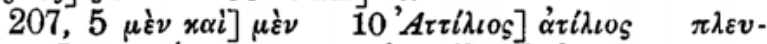

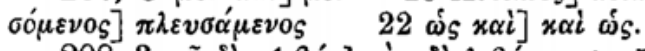

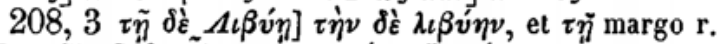

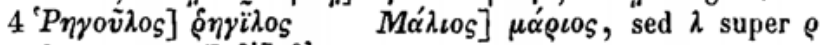
eadem $\mathrm{m}$. $7 \delta \dot{\varepsilon}] \delta^{\prime}$.

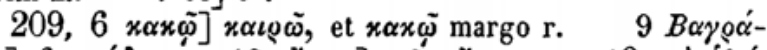

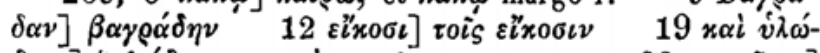

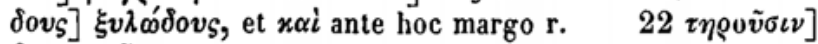

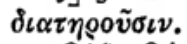

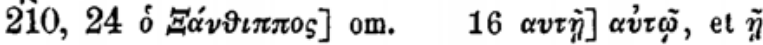


margo r. $\quad \hat{\varepsilon} \alpha v \tau o \tilde{v}] \hat{\varepsilon} \alpha v \tau$ cum ductu $\tau^{\tilde{2}}$, qui $\tau$ ov potius signi-

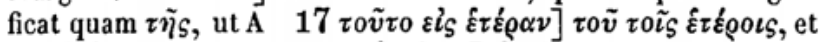

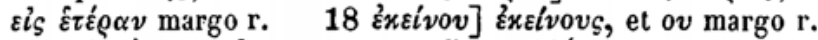

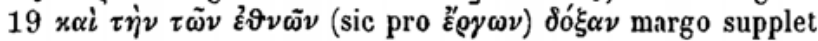
$\mathrm{m}$. antiquiori quam cetera $26 \pi 1 \lambda \alpha i \tau \iota v o \nu] \pi \lambda \alpha i ̈ \tau \iota \nu 0 v$ غ่ $\left.\varepsilon^{\prime} \pi \lambda \varepsilon v \sigma \alpha \nu\right] \dot{\alpha} \pi \dot{\varepsilon} \pi \lambda \varepsilon v \sigma \alpha \nu$, et $\varepsilon$ margo $\mathrm{r}$.

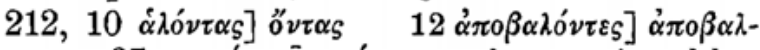

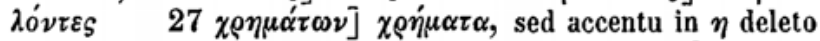
et in $\alpha$ addito et $\omega \nu$ super $\alpha$ posito $28 K \alpha \pi i \omega \nu] \pi i \omega \rho$ et $K \eta \pi i \omega \nu$ margo r. $29 \pi \varepsilon \iota \rho \alpha ́ \sigma \alpha \nu \tau \varepsilon \varsigma] \pi \varepsilon \rho \alpha ́ \sigma \alpha \nu \tau \varepsilon \varsigma$, et $\varepsilon \iota$ s. v. r. $\quad 31 \pi \alpha \rho \alpha \lambda i \alpha \nu] \pi \alpha \rho \alpha ́ \lambda \iota \nu \nu$.

$213,1 \tau \tilde{\omega} \nu \nu \alpha v \tau \iota \% \tilde{\omega} \nu] \tau \dot{o} \nu \alpha v \tau \iota x \grave{\nu} \nu$, et $\tau \tilde{\omega} \nu$ margo $\mathrm{r}$.

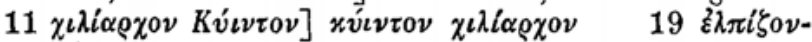

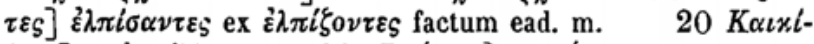

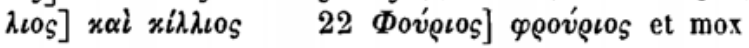
$v$

$28 \mu \alpha \chi 0 v \mu \varepsilon v 0 \iota] \mu \alpha \chi 0 \mu \varepsilon \nu 0 \iota$, sed ó eadem, ut videtur, $\mathrm{m}$.

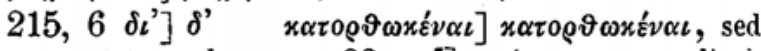
0 in $\omega$ mutato ead. m. 22 rov $] v$ in rasura ampliori, quasi $\tau 0$ ṽ $\tau$ fuerit.

216, $\left.19 \delta \delta^{\prime} \alpha v^{\prime} \dot{v} v\right] \delta^{\prime} \alpha \dot{v} \tau \dot{v} v$ bis in fine versus et initio sequentis. Ad alterum $\delta \iota$ margo r. $28 \lambda \varepsilon^{\prime} \gamma_{\varepsilon \iota}$ anguste insertum ead. $m$.

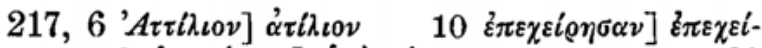

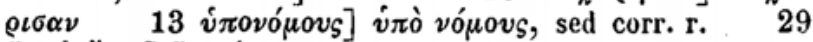

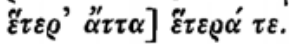

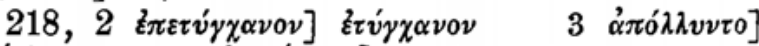

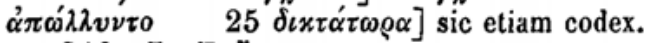

$219,7 \varepsilon i] \eta^{\eta}$.

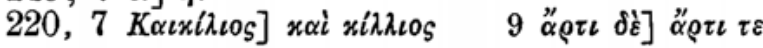
recte.

$221,24 x \alpha \tau \alpha \sigma \tau \eta \dot{\eta} \sigma 0 v \sigma \iota v] x \alpha \tau \alpha \sigma \tau \eta \dot{\sigma} \omega \omega \iota \nu \quad 29 K \alpha \varrho \chi \eta-$

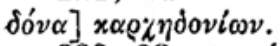

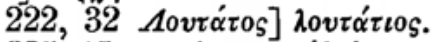

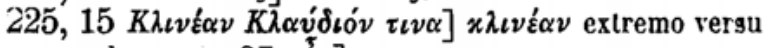
in marg. ead. m. 27 ov $v$ ] om., et s. v. r.

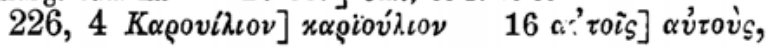




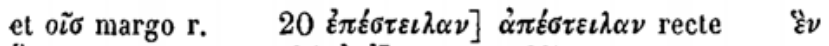
ö $v$, et $\varepsilon$ s. v. r. $\left.\quad 31 \varepsilon^{\prime} \pi^{\prime}\right]$ om., et addit margo r.

227, $\left.1 \sum \alpha \varrho \delta o ́ v \alpha \varsigma\right] \sigma \alpha \rho \delta \tilde{\omega} \nu \alpha \varsigma$.

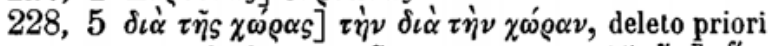
$\tau \dot{\eta} \nu$ et ascripto ad alterum $\tau \tilde{\eta} \sigma$ margo r. 15 है $\tau \iota]$ ó $\tau \iota$ corr. r. $\quad 31 \quad \delta \xi] ~ o m$, et addit margo r.

$229,4 \pi \varepsilon \pi \varepsilon \iota x o ́ \tau 0 \varsigma] \pi \varepsilon \pi \eta$ xótos $21 \delta v ́ \omega] \delta$ óo.

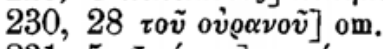

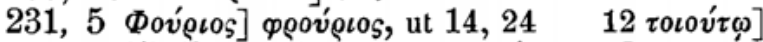
om. $\left.14 \delta^{\prime}\right] \delta \dot{z}$ ut Wolfius $27 M \alpha^{\prime} Q x \varepsilon \lambda \lambda_{0 s]}$ om.

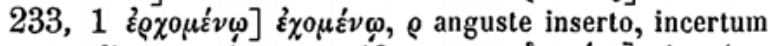
eadem an alia $\mathrm{m}$. antiqua $\left.19 \pi \varepsilon v \tau \varepsilon x \alpha \iota \delta \varepsilon x \alpha \varepsilon \varepsilon^{\prime} \eta\right]$ sic etiam codex ut edd. Paris. et Wolfii $23 \pi \varrho 0 \sigma x \alpha \tau \varepsilon \dot{\varepsilon} \alpha \beta \varepsilon]$ ita cod. 234, $7 \varepsilon \gamma i v \omega \sigma x \varepsilon v-x \alpha i$ $x \alpha \tau \dot{\alpha}$ ' $P$. $\alpha \dot{v} \tau \tilde{\omega} \sigma$.$] om., et addit$

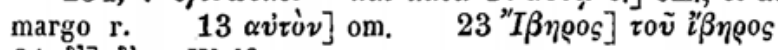
$\left.31 \delta^{\prime}\right]$ d ̀े ut Wolfius.

$235,1 \pi \alpha \varrho 2 \sigma x \varepsilon v \dot{\alpha} \alpha \varepsilon] \pi \alpha \varrho \varepsilon \sigma x \varepsilon v \dot{v} \alpha \sigma \varepsilon \nu$ ut idem

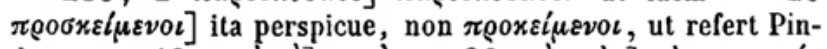

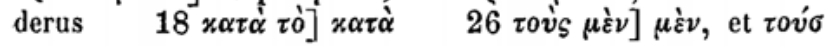
margo $r$.

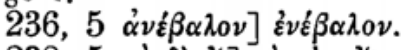

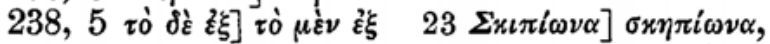

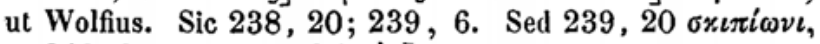
ut 240,9 et seqq. $27 \mu \dot{\varepsilon} \nu]$ om.

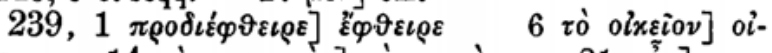

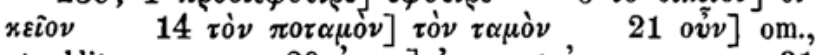
et addit margo r. $29 \stackrel{\alpha}{\alpha} \pi 0 \tau$.] $\varepsilon \pi \iota \tau$., et $\dot{\alpha} \pi \circ$ margo r. 31

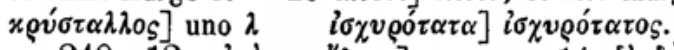

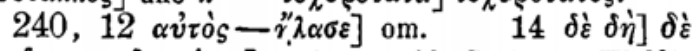

$\left.27 \varepsilon \tilde{\varepsilon} \tau \alpha x \alpha \iota \delta \varepsilon \% \alpha_{\varepsilon}^{\prime} \tau \eta_{i}\right]$ cod. ut edd. Paris. et Wolfii 30 $\delta \dot{\varepsilon}]$ om.

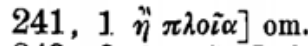

$243,8 \beta \alpha \nu \nu \tilde{\omega} \nu \alpha] \beta \alpha \alpha \nu \nu \tilde{\omega} \nu \alpha \quad 12[\alpha i t o v] \gamma \alpha \dot{i o v} \mathrm{ut}$

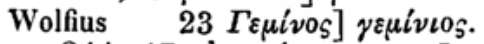

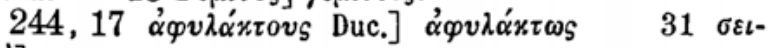
$\sigma \mu \mathrm{oi}] \mathrm{om}$.

$245,11 \Sigma \pi \omega \lambda \iota \tau i o v] \sigma \pi \omega \lambda \eta \tau l o v \quad 13 \Sigma \pi 0 \lambda \iota \tau i \omega]$

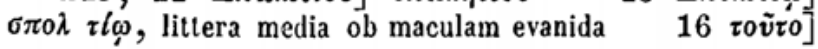




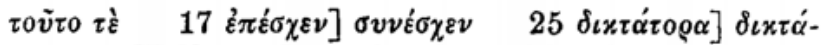
$\tau \omega \varrho \alpha$, ut Wolfius et Ducangius, hic et $31 ; 248,1,8,29$.

$\left.246,5 v \varepsilon v \iota x \eta x_{0} \alpha_{\varsigma}\right] v \varepsilon v \iota x u ́ r \alpha \alpha_{S}$ et $x \eta$ s. v. r.

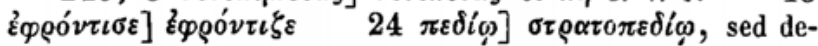
leto $\iota \mathrm{r}$.

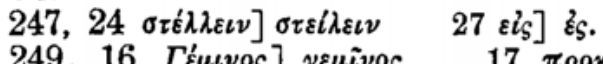

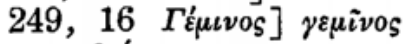
$\pi \rho 0 x \alpha \tau \omega \varrho \vartheta \omega ́ x \varepsilon$.

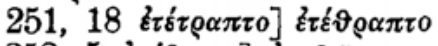

22 हैं

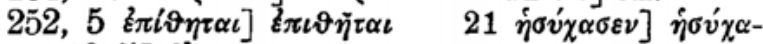
$\left.\zeta \varepsilon v \quad 23 \delta \delta^{\prime}\right], \delta^{\prime}$.

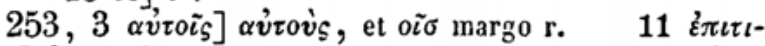
$\vartheta \tilde{\omega} \sigma \iota] \varepsilon \dot{\varepsilon} \pi \iota \varepsilon \vartheta \tilde{\omega} \sigma \iota \mathrm{pr}$. et $\iota$ super $\varepsilon \mathrm{r}$. Sed recipiendum $\varepsilon \pi \iota$ -

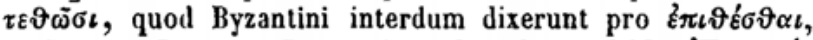
ut Ioannes Laurent. De magistr. 3, 53, p. 242: 'Ex $\tau \alpha v$ '

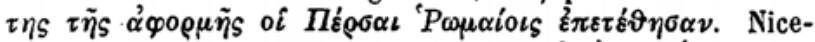
phorus Phocas De velit. bell. p. 149, A : 'E⿺่v $\pi \lambda \eta^{\prime} \vartheta \eta \pi$

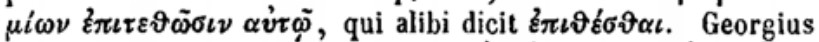

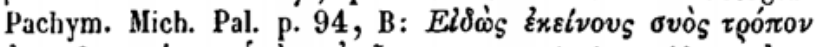

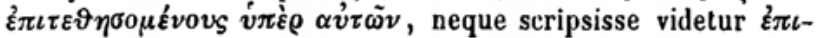
$\vartheta \eta \sigma 0 \mu \varepsilon \dot{v} v_{\varsigma}$, ut p. 349, A. Sic iidem dicunt $\pi \varrho 0 \sigma \tau \varepsilon \vartheta \tilde{\eta} \nu \alpha \iota$ et

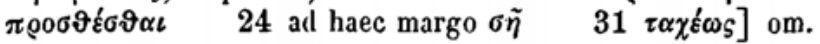

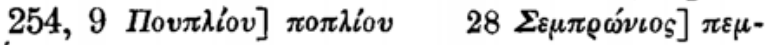
$\pi \varrho \omega ́ v ı$.

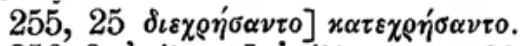

256, $\left.3 \dot{\eta} \mu \dot{\varepsilon} \lambda \eta \sigma \alpha \nu] \dot{\eta} \mu \dot{\varepsilon} \lambda \lambda \eta \sigma \alpha \nu \quad 28 \dot{\varepsilon} \pi \alpha \nu \alpha \sigma \tau \alpha_{s}\right] \dot{\alpha} \mid \pi \alpha-$ $v \alpha \sigma \tau \dot{\alpha} \varsigma$ addito initio alterius versus alia manu $\dot{\varepsilon}$, sed relicto

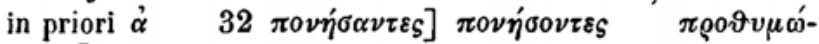
$\tau \varepsilon \rho \circ \nu]$ etiam hic codex ut ceteri.

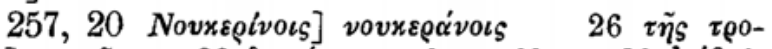
$\varphi \tilde{\eta} s] \tau \varrho \circ \varphi \tilde{\eta}_{S} \quad 28 \delta \iota x \tau \alpha^{\prime} \tau \omega \varrho \alpha$ cod. ut edd. $\quad 29 \varepsilon_{\varepsilon}^{\prime} \beta \alpha \lambda$ -

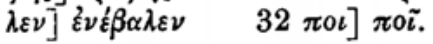

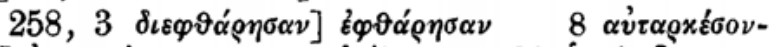

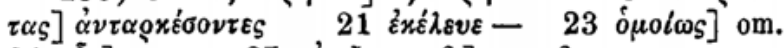

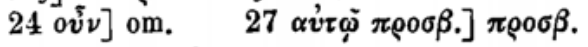

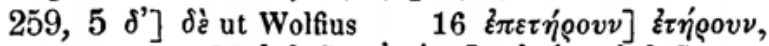
sed $\pi \varepsilon$ s. v. r.

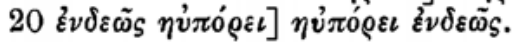




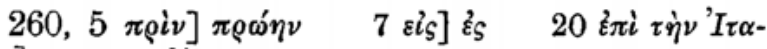
$\lambda i \alpha \nu]$ om., et addit margo $\mathrm{r}$.

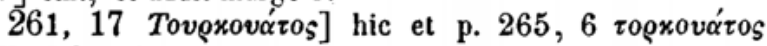
ut Vindob., forma et his locis a me recepta et antea, ubi iidem cum ceteris per $0 v$, restituenda $\left.28 \varepsilon \pi^{\prime}\right] \mu \varepsilon \tau$ ', et $\varepsilon^{2} \pi$ ' margo $\mathrm{r}$.

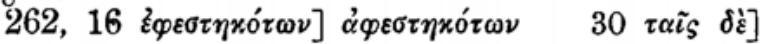
$\tau \alpha i \bar{\varsigma} \tau \varepsilon$.

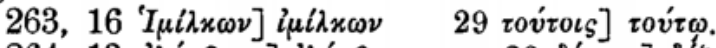

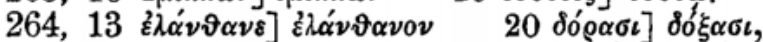

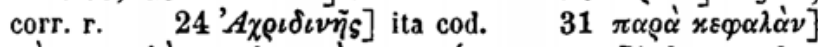

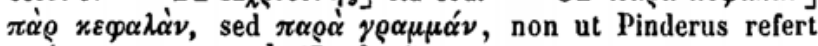

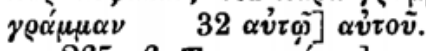

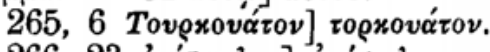

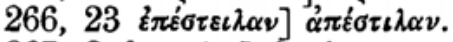

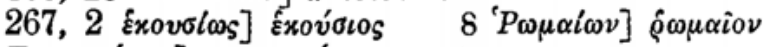

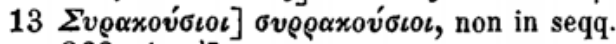

$268,1 \tau \dot{\alpha}]$ om.

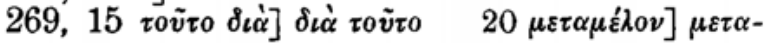
$\mu \varepsilon \dot{\lambda} \lambda o v$, et ov margo r.

$270,1 \dot{\alpha} \pi 0 x \iota v \delta v \nu \varepsilon \tilde{v} \sigma \alpha \iota] \delta \iota \alpha x \iota \nu \delta v v \varepsilon \tilde{v} \sigma \alpha \iota \quad 9 \Sigma \alpha \lambda \alpha-$ $\pi i \alpha \nu] \sigma \alpha \lambda \pi i \alpha \nu$.

271, $15 \tau \tilde{\eta} \tau \varepsilon \pi \rho \circ \beta \beta \lambda \hat{\eta}]$ om. , $\tau \tilde{\eta}]$ om., quod delen-

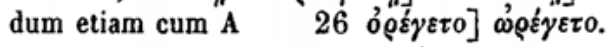

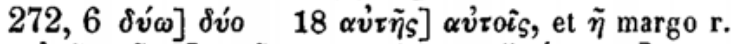

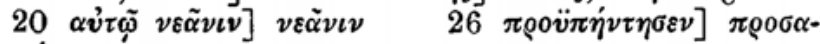
$\pi \dot{\eta} \nu \tau \eta \sigma \varepsilon v$.

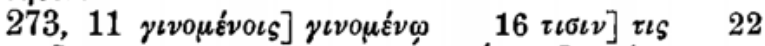

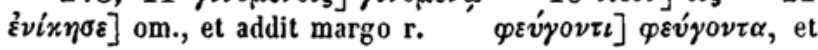
ı s. v. r.

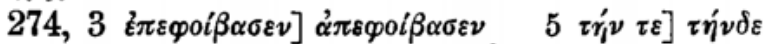

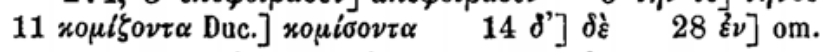

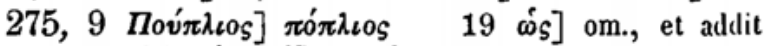

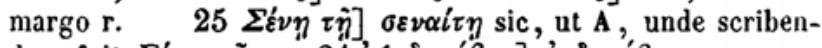

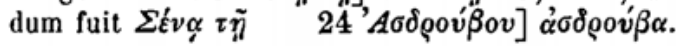

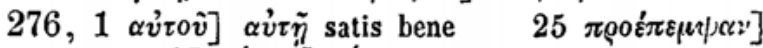

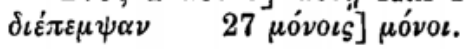




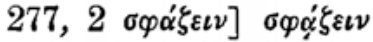
$v i \alpha \nu$, sed infra $\alpha \nu$ lineola $\mathrm{r}$.

$22 B \rho \varepsilon \tau \tau i \alpha \nu] \beta \varrho \varepsilon \tau \tau \alpha-$ $26 \delta \dot{v} \omega] \delta v^{\prime}$.

$278,7 \tau \tilde{\omega} \nu \Sigma x \iota \pi \iota v^{\nu \omega \nu]} \sigma x \iota \pi i \omega v$, corr. s. v. r. et ad-

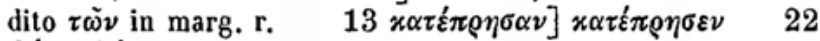
$\left.\delta v^{\prime} \omega\right] \delta$ v́.

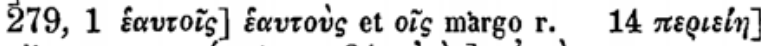

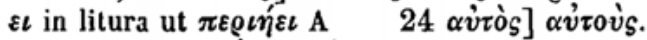

$280,4 \varepsilon i \pi \dot{\omega} \nu] l \pi \dot{\nu} \nu$ pr., addito $\left.\varepsilon \mathrm{r} . \quad 15 \mu \dot{\nu} \nu \tau \tilde{\eta}_{S}\right]$ $\left.\tau \tilde{\eta}_{S} \mu \dot{\varepsilon} \nu 270 \dot{v} \alpha \lambda \varepsilon \rho l \alpha \varsigma\right]$ ov $\alpha \lambda \lambda \varepsilon \rho i \alpha s$, ut idem cum cete-

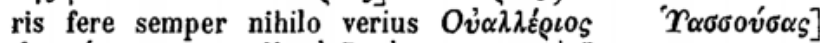

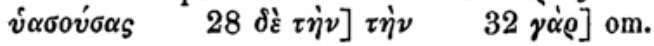

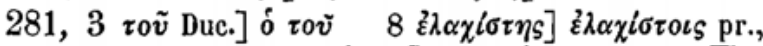
corr. ead. m. $17 M \alpha \sigma \iota v i \sigma \sigma \alpha \varsigma] \mu \alpha \sigma \alpha v i \sigma \sigma \alpha_{S} \quad 19 \Pi v$ -

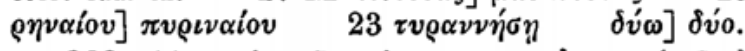

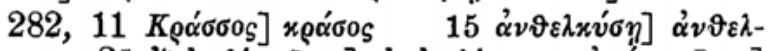

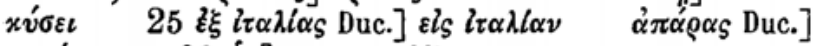

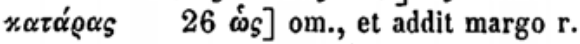

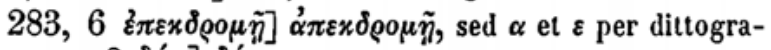
phiam $\left.8 \delta v^{\prime} \omega\right]$ ¿vio.

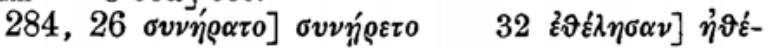
$\lambda \eta \sigma \alpha \nu$.

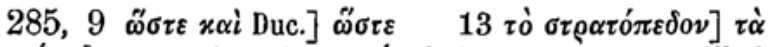
$\sigma \tau \varrho \alpha \tau o ́ \pi \varepsilon \delta \alpha$, quamvis retinens $\tau$ ó. Sed $o$ et ov s. v. r. Illud

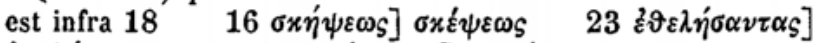

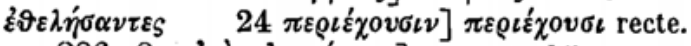

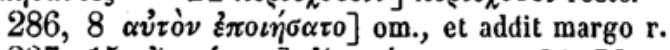

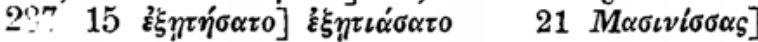
$\mu \alpha \sigma \alpha \nu i \sigma \sigma \alpha$ s.

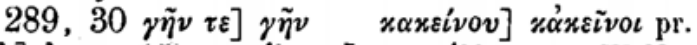

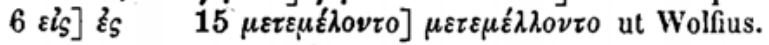

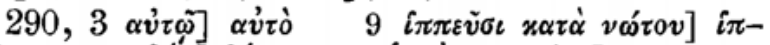
$\left.\pi \varepsilon v \tilde{\iota} \quad 17 \delta^{\prime} v^{\prime} \omega\right] \delta v^{\prime} \sigma \quad 23 \delta$ o $\left.\alpha^{\prime} \varrho \Sigma x \iota \pi i \omega v\right]$ om., et addit margo $r$.

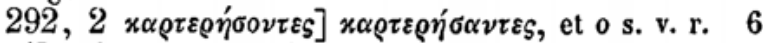
$\left.\delta \grave{\varepsilon} x \alpha i] x \alpha i \quad 27 \varepsilon i_{\zeta}\right] \dot{\varepsilon} s$.

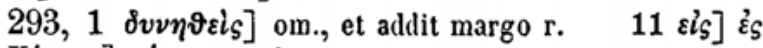

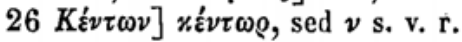




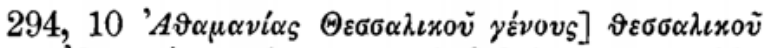
yévovS $\alpha \vartheta \alpha \mu \alpha v i \alpha_{S}$, sed cum numeris $231 \mathrm{r}$. supra positis.

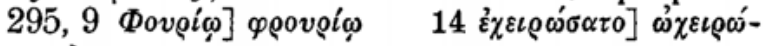
$\sigma \alpha \tau$, sed $\varepsilon$ s. v. r.

$\left.\left.296,15 \delta^{\prime}\right] \delta \dot{\varepsilon} \quad 21 \varepsilon i \varsigma\right] \dot{\varepsilon}_{S} \quad 23 \tau \tilde{\eta}_{S}$ Ko@.] $\tau \tilde{\eta}_{S} \tau \varepsilon$ xоอ. $\left.30 \dot{\eta} \tau 0 \iota \mu \alpha^{\prime} \xi \varepsilon \tau 0\right]$ om., et addit margo r.

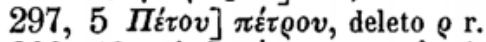

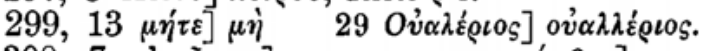

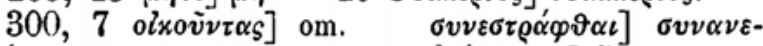

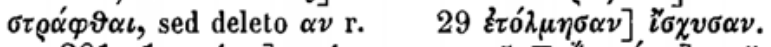

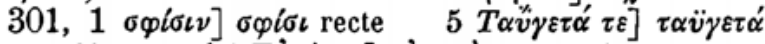
$\tau \varepsilon$ ut Wolfius $17 E \dot{v} \mu \dot{\varepsilon} v \eta \varsigma] \varepsilon \dot{v} \mu \varepsilon \nu \eta_{S}$ ut Wolfius, et infra uterque p. $307,26,28 ; 315,23$.

$302,23 \Lambda v \sigma \iota \mu \alpha \chi i \alpha \nu] \lambda v \sigma \iota \mu \alpha \chi i \delta \alpha$.

$303,2 \sigma \dot{v} \nu]$ om. et addit margo r. $7 \pi \rho \varepsilon \dot{\sigma} \beta \varepsilon \iota_{\varsigma}-$ $\dot{\alpha} \nu \tau \varepsilon \pi \dot{\varepsilon} \sigma \tau \varepsilon \lambda \lambda o \nu$ etiam hic codex, quod scribendum $\alpha \nu \tau \alpha \pi \varepsilon^{\prime} \sigma \tau \varepsilon \lambda-$

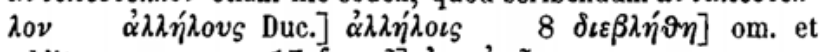
addit margo r. $17 \varepsilon \alpha v \tau \omega]]$ है $\quad \alpha \dot{v} \tau \tilde{\omega}$, corr. r.

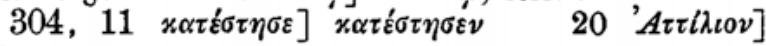

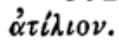

$305,1 \delta \dot{\varepsilon} x \alpha i] \delta \dot{\varepsilon} \quad 3 \gamma \dot{\alpha} \varrho]$ om., et addit margo r.

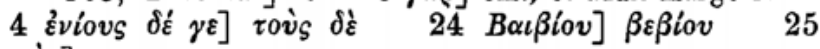

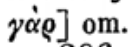

306, $7 \Gamma \lambda \alpha \beta \rho i \omega \nu] \gamma \varrho \alpha \beta \rho i \omega \nu$, et $\lambda$ r., sed $22 \gamma \alpha \beta \varrho i \omega \nu$. $307,7 \delta \dot{\varepsilon}]$ om., et s. v. addit $r$.

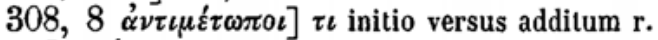

24

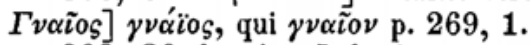

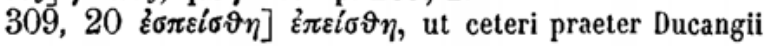
libros.

$\left.310,1 \pi \dot{\varepsilon} \mu \psi \alpha_{\varsigma}\right]$ हैं $\pi \varepsilon_{\mu} \psi \alpha_{S}$ sic 13 ó $\left.\tau \iota \delta \dot{\varepsilon}\right]$ ö $\tau \iota \tau \dot{\alpha}$, quod ó $\tau \iota \tau \varepsilon$ esse putabat Pinderus $18 \Pi \iota \sigma \iota \delta i ́ \alpha \nu] \tau o ́ \tau \varepsilon$

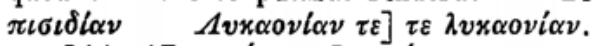

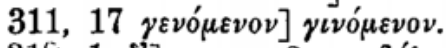

$\left.31 \%, 1 \delta^{\prime}\right]$ om. $\left.\quad 2 \mu \varepsilon \tau \alpha \beta \alpha^{\prime} \lambda o \nu \tau o\right] \mu \varepsilon \tau \varepsilon \beta \alpha^{\prime} \lambda o v \tau o$ $\tau \alpha \bar{\imath}_{S} \delta \dot{\varepsilon}$ Duc.] $\tau \alpha \hat{i} \sigma \delta \varepsilon$ ut Wolfius.

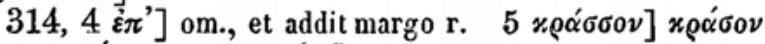

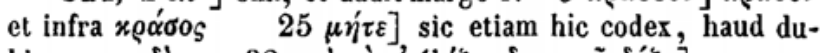

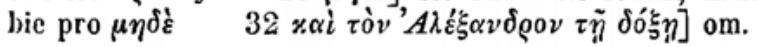




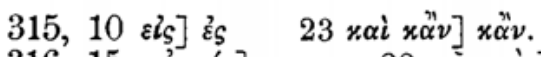

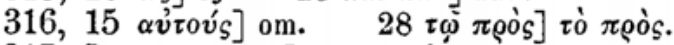

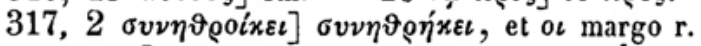

$\left.25 \pi \varepsilon \varrho \iota \imath \iota \pi \varepsilon \tilde{i}_{S}\right] \pi \varepsilon \varrho \iota \lambda o \iota \pi \varepsilon i_{S}$, deleto o r. $\left.28 \dot{\eta} \rho \tilde{\eta} \sigma \vartheta \alpha \iota\right] \dot{\eta}$ et suppleto in fine versus $\rho \tilde{\eta} \sigma \vartheta \alpha \iota$.

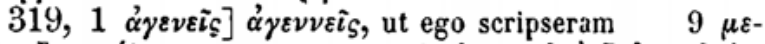

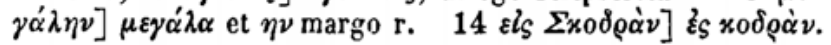

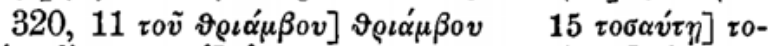

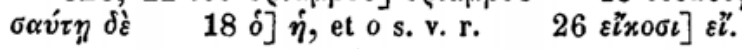

321,8 et $\left.11 \varepsilon \dot{v} \mu \varepsilon \nu \eta_{S}\right]$ ita codex hic et infra, etiam

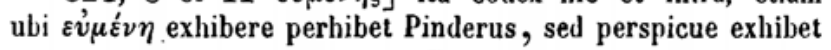

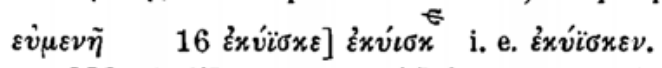

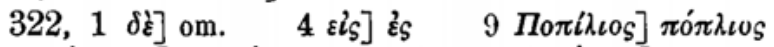

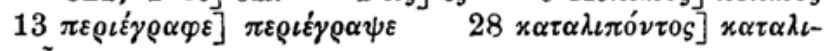
$\pi \tilde{\omega} \tau \sigma_{S} \mathrm{pr}$.

$\left.323,1 \delta \iota \varphi^{\prime} x o v \nu\right] \delta\llcorner\omega ́ x$ supra scripto $\eta \sigma \alpha \nu$ manu diversa, sed non recentiori illa quae pleraque ascripsit nec pallido qua illa utitur atramento.

$325,5 \sigma \pi o v \delta \dot{\alpha} \varsigma] \sigma \pi \varepsilon i \sigma \alpha \sigma \vartheta \alpha \iota$, sed excepto $\sigma \pi$ in litura, et $\sigma \pi 0 \nu \delta \dot{\alpha} \varsigma$ margo r. $7 \sigma \pi \varepsilon i \sigma \alpha \sigma \vartheta \alpha \iota] \sigma \pi 0 \nu \delta \alpha \dot{\alpha}$, et $\sigma \pi \varepsilon i \sigma \alpha-$ $\sigma \vartheta \alpha \iota$ margo r. $\left.9 \sigma \pi 0 \nu \dot{\alpha} \alpha_{\varsigma}\right] \sigma \pi \varepsilon i \sigma \alpha \sigma \vartheta \alpha \iota$, sed $\sigma \pi 0 \nu \delta \dot{\alpha} \varsigma$ ead. manu, ut videtur, in margine.

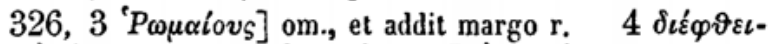

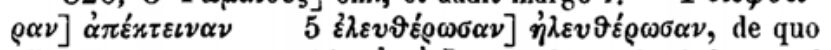
nihil Haasius ex A $\left.14 \alpha v \tau_{0} \dot{v}_{S}\right]$ etiam hic codex habet quod Wolfius et Ducangius, sed excidit apud Pinderum.

$\left.\left.327,10 \quad \varepsilon i_{\zeta}\right] \xi_{\xi}, \quad \delta^{\prime}\right]$ om., et addit margo r. 14 oi $]$

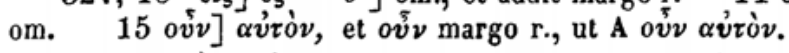

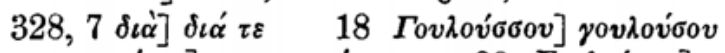

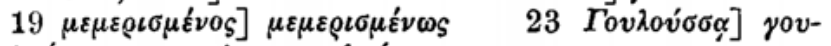

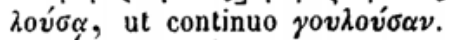

$329,13 \delta \dot{\varepsilon}]$ om. $18 \tau \varepsilon$ Duc.] $\delta \dot{\varepsilon}$.

$\left.\left.330,28 K \alpha \iota x i \lambda_{\iota} \sigma \nu\right] x \alpha i x i \lambda_{\iota} \sigma \nu \quad 30 x \alpha i\right] x \alpha i$ oi, ut Wolfius, quod restitui, etsi ad Parisinae $x \alpha i$ tacet Haasius apud Pinderum, qui cum Parisina $x \alpha i$, altero non memorato.

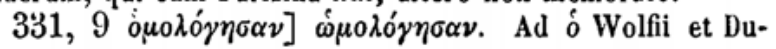


cangii tacet Haasius apud Pinderum, qui $o$ $12 \tau \dot{\partial} \nu] \tau \dot{j}$, et s. v. ead. m. $\nu$.

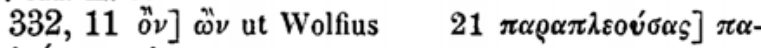
$\varrho \alpha \pi \lambda \varepsilon v ́ \sigma \alpha \alpha_{\varsigma}$, sed 0 s. v. r.

$333,28 \not ̈ \nu \tau \iota x \rho v] \dot{\alpha} \nu \tau \iota x \rho v ่$

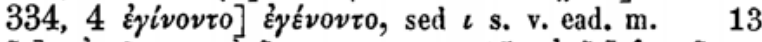
$\left.\alpha \hat{v} \tau \tilde{\eta}_{S}\right] \alpha \hat{v} \tau o \tilde{i}_{S}$, et $\alpha \dot{v} \tau \tilde{\eta}_{S}$ margo r. $\left.15 \alpha \dot{v} \tau \tilde{\eta}_{S}\right] \hat{\varepsilon} \alpha v \tau \tilde{\eta}_{S}$

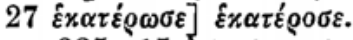

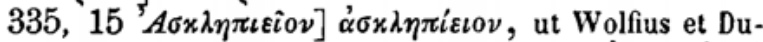
cangius, quod tacito Pinderus correxit $\dot{\alpha} v \varepsilon \lambda \eta \lambda \hat{v} \vartheta \varepsilon \iota]$ $\dot{\alpha} \nu \varepsilon \iota \lambda \eta^{\prime} \vartheta \varepsilon \iota, \mathrm{ut} \mathrm{A} \dot{\alpha} \nu \varepsilon \iota \lambda \eta^{\vartheta} \vartheta$.

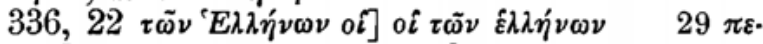

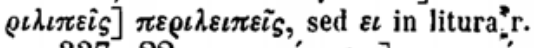

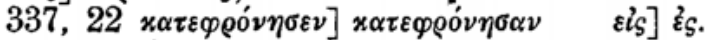

$338,5 \tau \alpha^{\prime}$ Duc.] $\tau \alpha^{\prime} \tau \varepsilon$.

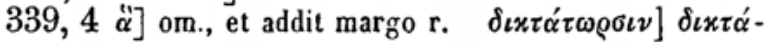
$\tau 0 \rho \sigma \iota$, sed 0 in $\omega$ mutato $\mathrm{m}$. antiqua et fortasse eadem, quum etiam p. 339, $19 \delta \iota x \tau \alpha \tau \omega \varrho \omega \nu$ perspicue sine ulla mutatione habeat codex, quod ego constanter exhibui et confirmavi

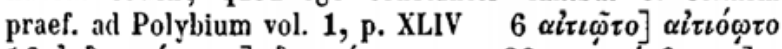

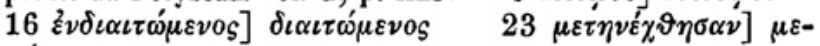
$\tau \eta^{\prime} \chi \vartheta \eta \sigma \alpha \nu$.

340,1 Sequentibus rubro col. et litteris maioribus superscriptum:

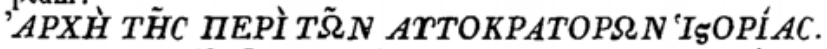

$3 \tau v \varrho \alpha v v i \delta o s] \tau v \rho \alpha v v i \delta$, sed $0 \sigma$ s. v. r. $\quad 4 \delta \iota x \tau \alpha \dot{\tau} \omega \rho-$ $\sigma \iota \nu]$ ita cod.

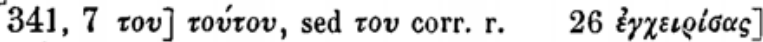

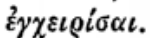

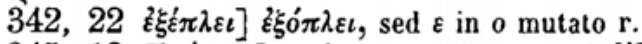

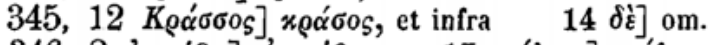

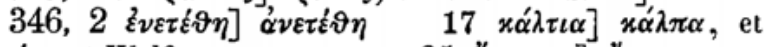
$x \alpha \lambda \tau i \alpha$, ut Wolfius, margo r. $\left.25{ }^{\prime \prime} \nu \tau \iota x \rho v\right]$ ¿ $\nu \tau \iota x \rho v \varsigma$ ut

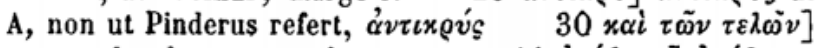

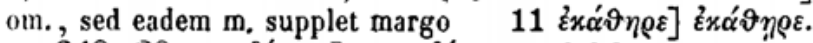

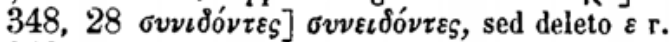

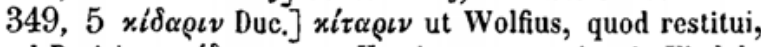
etsi ad Parisinae $x i \delta \alpha \rho \iota v$ tacet Haasius, quum etiam in Vindob. 


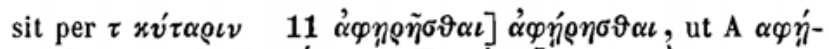
$\varrho \eta \sigma \vartheta \varepsilon \quad 12 \Sigma \omega \varphi \eta \dot{\nu} \nu \nu-\Sigma \omega \varphi \eta \dot{\nu} \nu \varsigma] \sigma \omega \varphi \eta v \dot{\eta} \nu-\sigma \omega \varphi \eta-$ $v \tilde{\eta}_{\mathrm{s}}$, ut voluerat Pinderus, quem fugit hoc esse in codice

$$
0
$$

$\left.28 \pi \varepsilon_{\mu}^{\prime} \psi \alpha \nu \tau \iota\right] \pi \varepsilon_{\mu}^{\prime} \psi \alpha \nu \tau$, sed $\iota$ s. v. r.

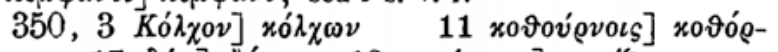

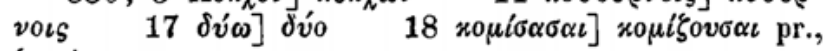
$\zeta o v$ in $\sigma \alpha$ mutato $\mathrm{r}$.

$351,6$ " $A \mu \alpha \nu 0 \nu]$ etiam hic codex ut Wolfius' et Duc., quod hic sine libris correctum, supra p. 21, A ex A 14

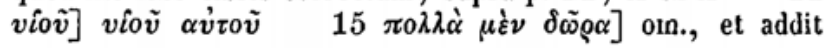
margo $\mathrm{r}$.

352, $17 \pi \alpha \varrho \varepsilon \sigma x.] \pi \alpha \rho \alpha \sigma x . \quad 32 \tau \alpha \mu \varepsilon i \tilde{0} \nu] \tau \alpha \mu \iota \varepsilon \tilde{i} 0 \nu$.

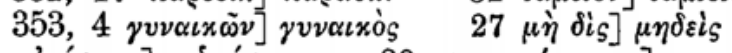

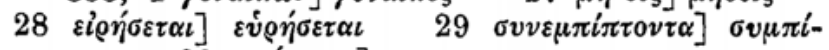
$\pi \tau 0 \nu \tau \alpha \quad 30 \gamma \varepsilon v o ́ \mu \varepsilon v 0 \varsigma]$ om.

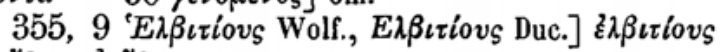

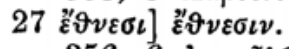

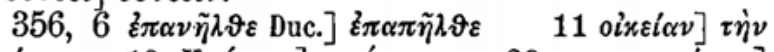

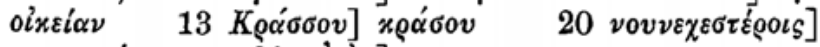

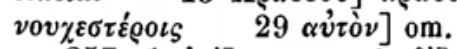

$357,1$ kं $\pi i]$ om. $3 \delta \dot{\xi}] \delta$ ' ut Wolfius et ego, etsi ad $\delta \dot{\varepsilon}$ tacet Haasius $\left.\pi 0 \lambda \lambda_{0} v_{\varsigma}\right]$ om. $\left.18 \alpha^{\alpha} \xi \iota \iota^{\prime} \nu \tau \omega \nu\right]$ $\left.\begin{array}{cccc}\alpha \xi \iota 0 \tilde{v} \sigma \vartheta \alpha \iota & 23 \delta v \circ \tilde{\iota} \nu] \delta v \varepsilon \tilde{\nu} \nu & 26 & \mu \varepsilon \tau \eta \mu \varphi \iota \alpha \sigma \mu \varepsilon v_{\nu} \iota\end{array}\right]$ $\mu \varepsilon \tau \alpha \mu \varphi \iota \alpha \sigma \mu \varepsilon \dot{v} \nu \iota$, sed $\eta$ super $\alpha \mu$ ead. m. $\left.28 \varepsilon \varepsilon^{\prime} \lambda o \gamma i \mu o v s\right]$

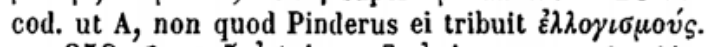

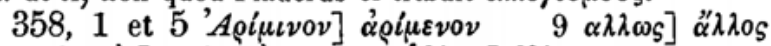

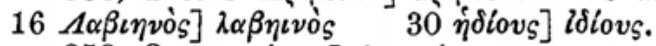

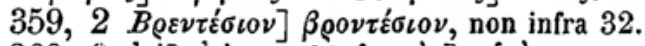

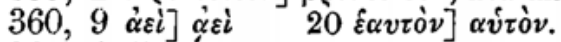

$361,4 \delta \dot{\varepsilon}]$ om.

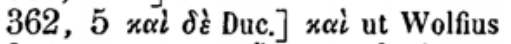

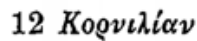

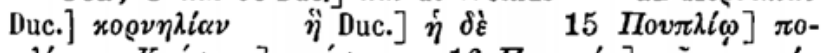

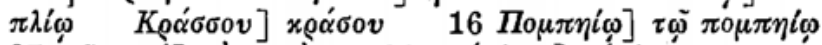

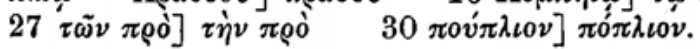

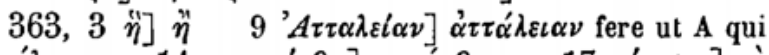

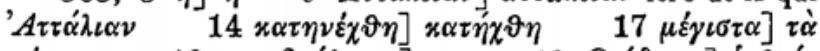

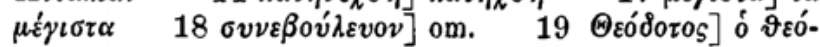




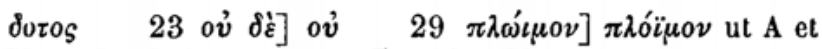
Plutarchi plerique, recte. Ex eodem Plutarcho mox $\pi \rho 0 \sigma \varepsilon \mu-$ $\beta \tilde{\eta} v \alpha \iota$ mutandum videtur in $\pi \rho 0 \varepsilon \mu \beta \tilde{\eta} \nu \alpha \iota$. Nam ipse post illos

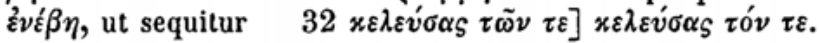

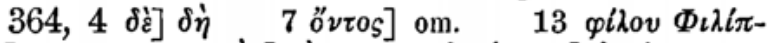

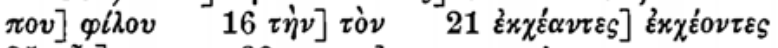

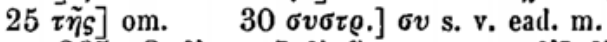

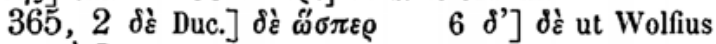
$24 \mu \iota x \rho \grave{\nu} \nu$ om.

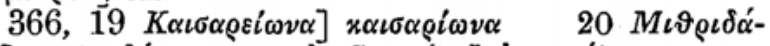

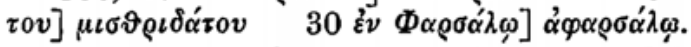

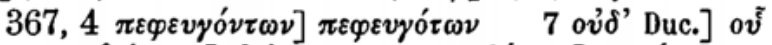

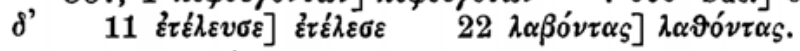

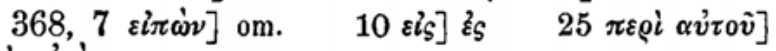

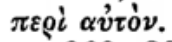

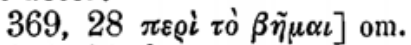

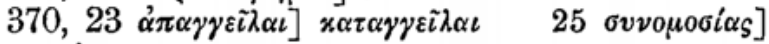

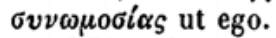

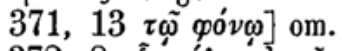

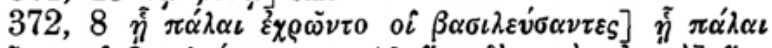

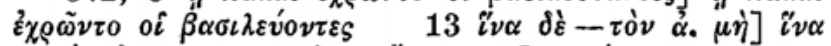

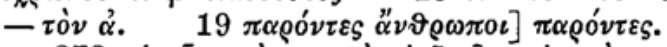

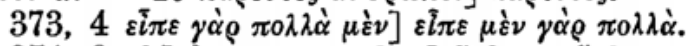

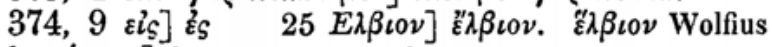

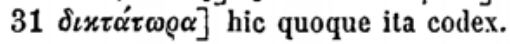

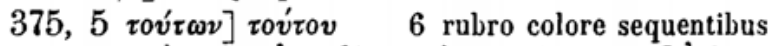

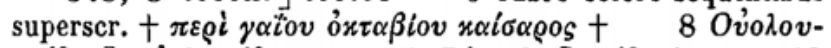

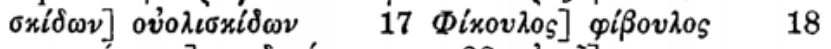
$\pi \iota \sigma \tau \varepsilon v o ́ \mu \varepsilon v 0 \varsigma] \pi \alpha \iota \delta \varepsilon v o ́ \mu \varepsilon v o s \quad 23 \alpha v \tau o v$ ] om.

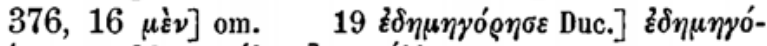

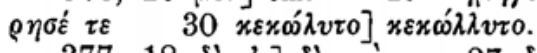

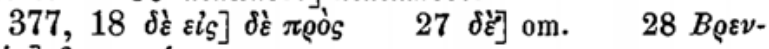

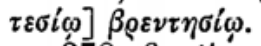

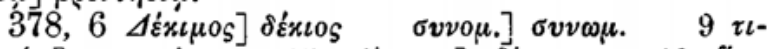

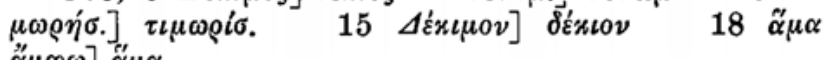

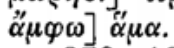

$\left.379,16 \varepsilon_{\lambda} i \xi \alpha \nu \tau \varepsilon \varsigma\right]$ ita cod. et infra $\left.I \varrho \tau.\right] i \varrho \tau$. 
$\left.380,8 \stackrel{\alpha}{\alpha} \pi \alpha \lambda \lambda \alpha^{\prime} \xi \alpha \nu \tau 0 \mathrm{~s}\right] \stackrel{\alpha}{\alpha} \pi \eta^{\prime} \lambda \lambda \alpha \xi \alpha \nu$

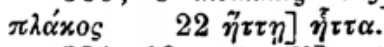

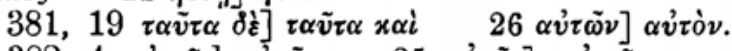
$\left.\left.382,4 \alpha \dot{v} \tau o \tilde{\iota}_{S}\right] \alpha \dot{v} \tau \tilde{\eta}_{S} \quad 25 \alpha \dot{v} \tau \tilde{\eta}_{S}\right] \alpha \hat{v} \tau \tilde{\iota}_{S}$ pr. correctus eadem manu.

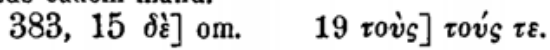

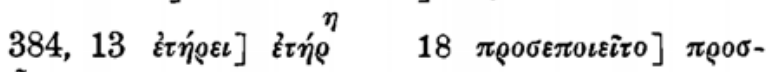

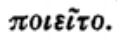

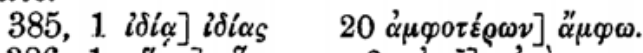

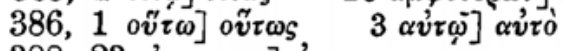

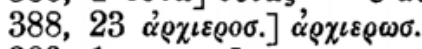

$389,1 \pi \rho \circ \alpha \alpha \tau$.] $\pi \varrho о \sigma \alpha \alpha$. ov้ $\quad 11 \mu \eta^{\prime} \tau \varepsilon$ Duc.] $\mu \eta^{\prime} \tau \varepsilon \tau \iota$

5 o $\pi 0 \sigma \omega \nu 0 \tilde{v} \nu]$ o $\pi 0 \sigma 0 \nu$ -

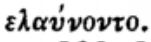

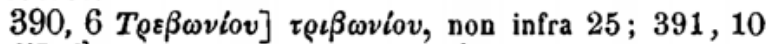

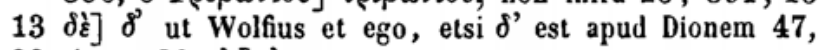
$\left.22,4 \quad 28 \varepsilon l_{S}\right] \varepsilon_{S}$.

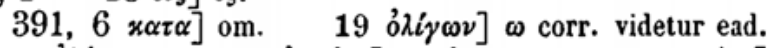

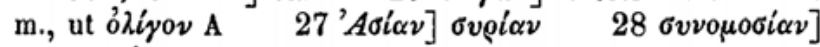
$\sigma v \nu \omega \mu \sigma \sigma i \alpha \nu$ ut ego.

$\left.392,10 \delta^{\prime}\right] \delta \dot{\varepsilon}$ ut Wolfius et ego $\left.18 \dot{\alpha} \pi \dot{\varepsilon} \lambda v \sigma \varepsilon\right]$

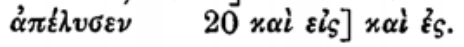

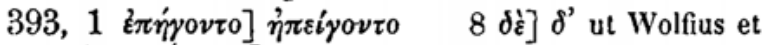
ego $24 x \alpha \vartheta \alpha \rho \sigma \iota \nu]$ punctum post hoc extritum. Inserendum vero $\tau \grave{o}$ ex Dione 32 żv $\tau \tilde{\eta}] \tau \tilde{\eta}$.

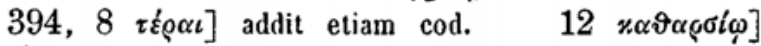
x $\alpha \rho \sigma i \omega$.

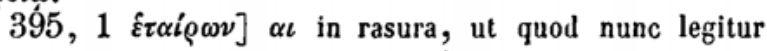
$\alpha$

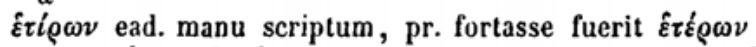

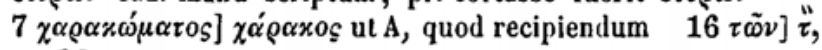
ut 20.

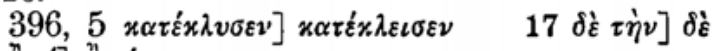

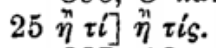

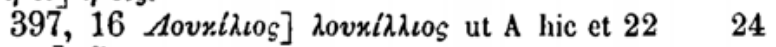
भ̆

$398,23 \varphi \alpha \sigma i] \varphi \eta \sigma i$. 


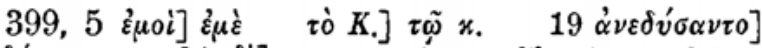
$\left.\alpha \nu \varepsilon \delta \alpha^{\prime} \sigma \alpha \nu \tau 0 \quad 21 \delta \xi\right] \tau \varepsilon$ pr. scripserat librarius, sed ipse correxit $25 \delta \dot{\varepsilon}] \delta^{\prime}$ ut Wolfius et ego.

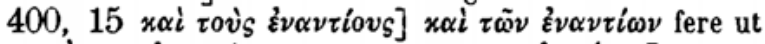
A, qui $\tau \dot{\alpha} \tau \tilde{\omega} \nu \varepsilon \dot{\varepsilon} \nu \nu \tau i \omega \nu$ cum Dione $\left.25 \dot{\varepsilon} x \varphi \eta^{\prime} \nu \alpha s\right]$ om.

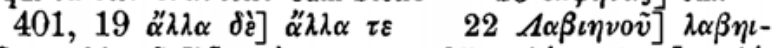

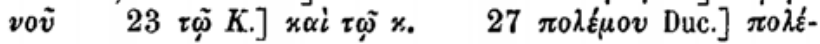
$\mu 0 v \tau \varepsilon$.

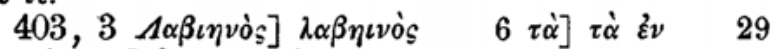

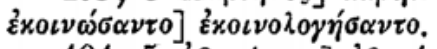

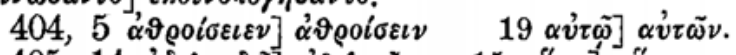

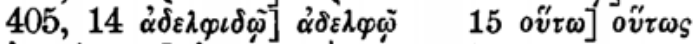

$\left.18 \dot{\alpha} \pi 0 \pi l \mu \pi \lambda \alpha_{S}\right]_{\alpha}^{\alpha} \pi 0 \pi \iota \mu \pi \lambda \dot{\alpha}_{S}$ ut apud Dionem, ex quo Zona-

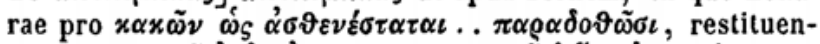

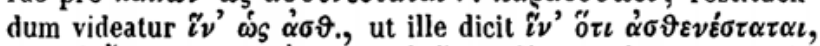

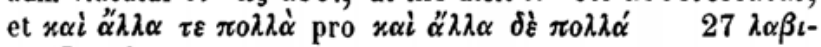
$\eta \nu \omega]] \lambda \alpha \beta \eta \iota \omega{ }^{\circ}$.

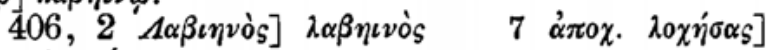
vं $\pi 0 \chi . \lambda \circ \gamma \chi \dot{\eta} \sigma \alpha s$.

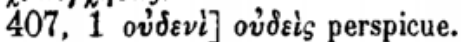

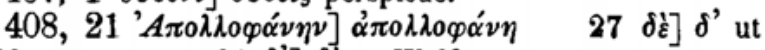
Wolfius et ego $\left.30 \delta^{\prime}\right] \delta \varepsilon$ ut Wolfius.

$409,2 x \lambda \omega \dot{v} \iota v$ etiam hic cod. ut libri Dionis, male pro

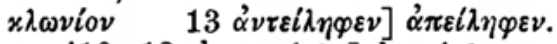

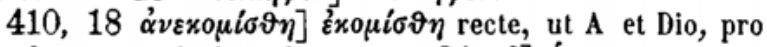
quo alterum me invito relictum $21 \tau \tilde{\omega}]$ ஸs.

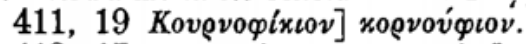

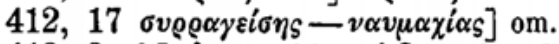

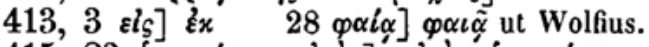

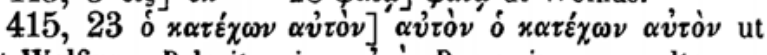
A et Wolfius. Delevit prius $\alpha \dot{v} \tau \dot{v} v$ Ducangius, ego alterum, nisi utrumque servandum, quantumvis post duo praecedentia molestum.

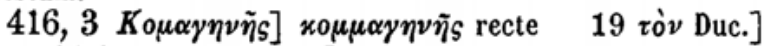
$\delta \dot{\varepsilon} \quad 23$ hic et $27 \quad \Sigma \tau \rho \alpha \tau$.] $\sigma \tau \alpha \tau$.

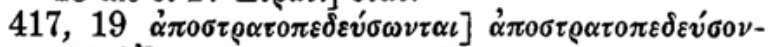

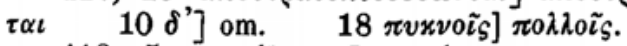

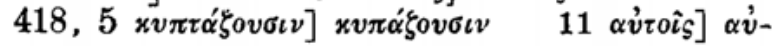
ZONARAS V. 


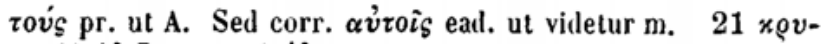

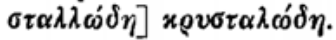

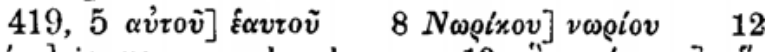

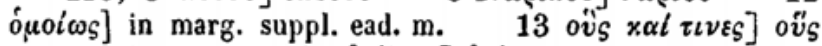

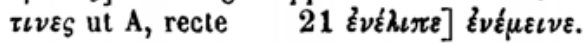

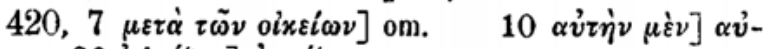

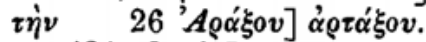

$421,9 \mu \dot{\varepsilon} \nu]$ s. v. ead. $m$.

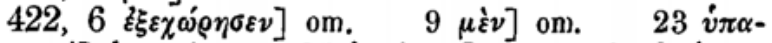

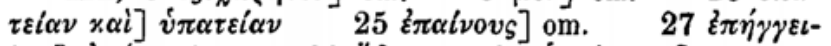

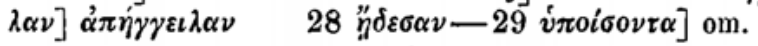

$423,1 \delta \dot{1}]$ om. $\left.\quad 2 \tau \dot{\alpha} S \delta i x \alpha_{S} \tau \varepsilon\right] \tau \dot{\alpha}_{S} \delta \iota \alpha \vartheta \eta^{\prime} \alpha_{S} \tau \varepsilon$. ldem perspicue $\sigma v \nu \varepsilon \xi \eta \dot{\eta} \tau \zeta \xi$, non quod Pinderus diserte ei tri-

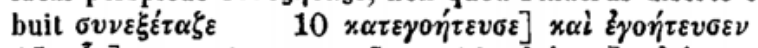
$15 \circ v^{3} \nu$ ] om. et in marg. $\left.\sigma \tilde{\eta} \quad 16 \alpha i \tau i \omega \mu \alpha\right] \alpha i \tau i \alpha \mu \alpha$ ut A. Idem vitium in Actis Apost. 25, 7 plurimos occupavit libros et editiones, etsi nullum est verbum unde duci possit $\quad 29$

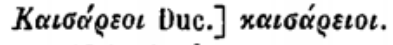

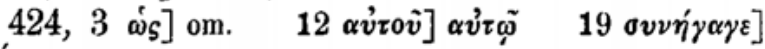
$\varepsilon i \sigma \eta^{\prime} \gamma \alpha \boldsymbol{\gamma}$.

$425,31 i \pi \pi 0 \mu \alpha \chi i \alpha] \nu \alpha v \mu \alpha \chi i \alpha$.

426,1 ì] $x \alpha i \dot{\alpha}$.

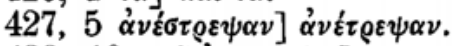

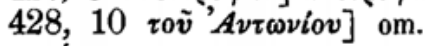

$12 \pi \varrho \circ \sigma \varepsilon \chi \omega \dot{\emptyset} \eta \sigma \alpha \nu]$

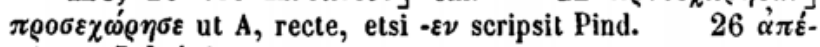
$\pi \lambda \varepsilon v \sigma \varepsilon \nu] \dot{z} \pi \dot{\varepsilon} \pi \lambda \varepsilon v \sigma \varepsilon \nu$.

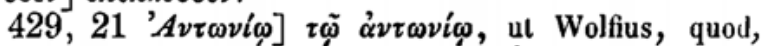
quum etiam apud Dionem $51,6,6$ sit $\alpha \pi \varepsilon x \rho i \nu \alpha \tau o ~ \tau \omega \tilde{~} \mu \dot{\varepsilon} \nu$

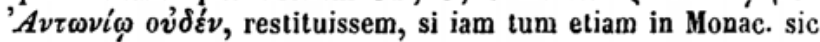
scriptum nossem. Nam $\tau \tilde{\omega}$ omissum apud Ducangium exstare in A fugisse videtur Haasium, etiam ad alia Parisinae vitia tacentem $22 \tau \tilde{\eta} \delta \dot{\varepsilon}-\dot{v} \pi \dot{\varepsilon} \sigma \chi \varepsilon \tau 0]$ om.

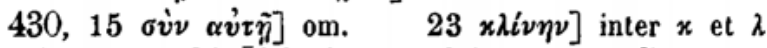
erasa littera 31 [delendum quod invito me relictum ex priori scriptura $\tau \tilde{\omega} \nu]$

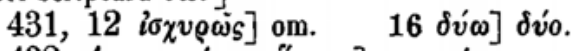

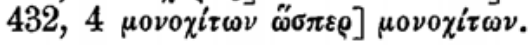




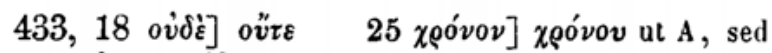
ut $v$ ex $v$ factum videatur.

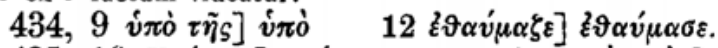

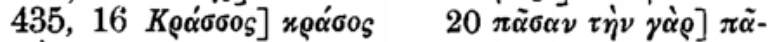
$\sigma \alpha \nu \tau \dot{\eta} \nu$.

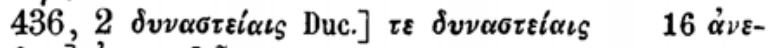
$\pi \dot{\alpha} \chi \vartheta \omega \varsigma] \dot{\alpha} \nu \varepsilon \pi \alpha \chi \vartheta \tilde{\omega}$.

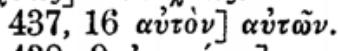

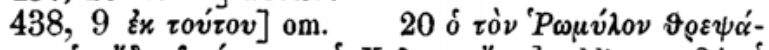

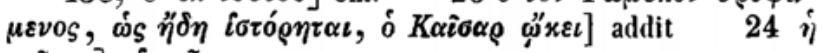
toĩ $\tau$.] of $\tau$ oĩ $\tau$.

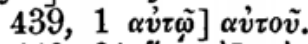

$440,31$ ö $\tau i \mu \grave{\eta}] \mu \dot{\eta}$ s. v. ead. m.

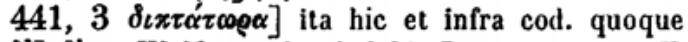

$\left.14 \delta^{\prime}\right] \delta \dot{\varepsilon}$ ut Wolfius et haud dubie Par., etsi tacet Haasius, ut saepius ad talia.

$442,20 \dot{\varepsilon} \pi \varepsilon \dot{i} \delta \dot{\varepsilon}]$ ad haec margo $\sigma \tilde{\eta}$.

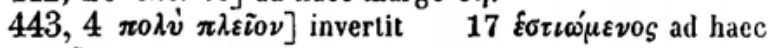
margo $\sigma \dot{\eta}$.

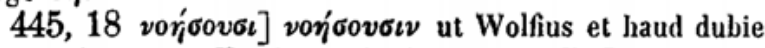
A, quamvis tacente Haasio, qui minuta nonuulla Parisinae vitia non animadvertisse videtur 19 ov̋ $\tau \omega]$ ov̉ $\pi \omega$.

446, $25 \dot{\varepsilon} v$ ] om. ut A et codices Dionis, ubi 55, 7, item

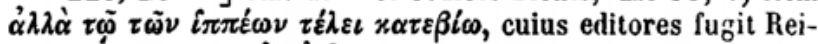
mari coniecturum $\dot{\alpha} \lambda \lambda^{\prime} \dot{\varepsilon} \nu$ a Zonara potuisse peti, etsi apud illum quoque mirum est $\varepsilon^{2} v$ omittere A et Monacensem, ut, nisi sit in Vindobonensi, Wolfii tantum editione nitatur.

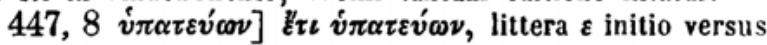
rubro colore scripta.

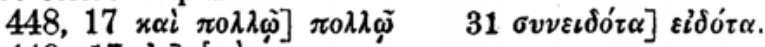
$449,17 \quad \xi x]$ viò.

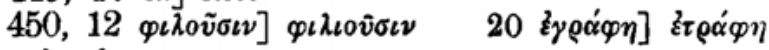
pr., sed ead. m. corr.

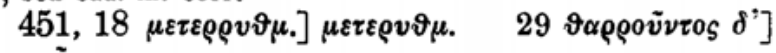

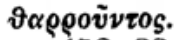

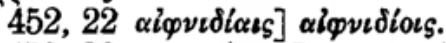

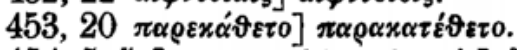

$454,5$ हैंti] om. $24 \Delta \alpha \lambda \mu \alpha \tau i \eta \eta]] \delta \alpha \lambda \mu \alpha \tau i \alpha$. 
456, $21 \tau \alpha \gamma \mu \alpha \dot{\tau} \omega \nu] \pi \varrho \alpha \gamma \mu \alpha^{\prime} \tau \omega \nu$.

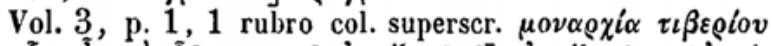

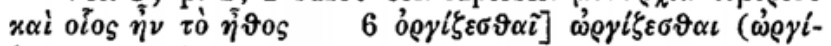

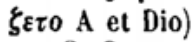

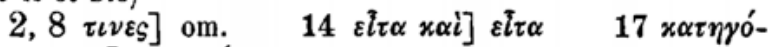
$\varrho \eta \sigma \alpha \nu$ Duc.] $x \alpha x \eta \gamma o ́ \varrho \eta \sigma \alpha \nu$ ut Wolfius.

$3,6 x \alpha i \lambda i \alpha v] \lambda i \alpha \nu \quad 25$ ad marg. $\sigma \tilde{\eta} \quad 32$ voбov́v$\tau 0 \varsigma$ ] $\nu 0 \sigma 0 \tilde{v} \nu \alpha s$.

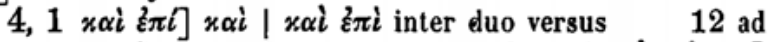
haec margo $\sigma \tilde{\eta}$, ut paullo post ad 1321 rov Av́rovírov]

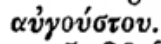

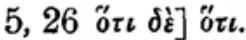
6, $26 \delta v \nu \alpha \tau \tilde{\omega} \nu] \delta v v \alpha \sigma \tau \tilde{\omega} \nu$

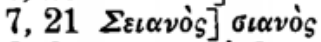
$\left.31 \pi 0 \lambda \lambda_{0} i\right] \pi 0 \lambda \dot{v}$.

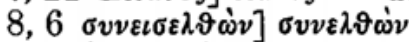
$19 \dot{\eta}$ om. Duc.] habet.
$21 \alpha \dot{v} \tau \tilde{\eta}] \alpha \dot{v} \tau \omega ั$

$\left.31 \sum \varepsilon i \ddot{\alpha} \nu \dot{\nu} v\right] \sigma \iota \alpha \nu o \dot{\nu}$.

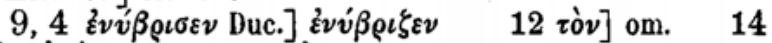

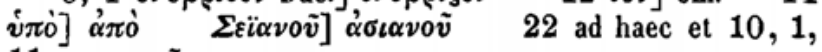
11 margo $\sigma \tilde{\eta}$.

$10,13 \alpha v \dot{v} \varepsilon v \tau i \alpha] \alpha v \tau o \varepsilon v \alpha v \tau i \alpha$, i. e. $\alpha \dot{v} \tau 0 \varepsilon v \tau i \alpha$ ut $\mathrm{A}$

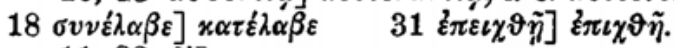

$\left.11,23 \quad \delta^{\prime} \dot{\varepsilon}\right]$ om.

$13,26 \delta v ́ \omega] \delta$ v́o.

$14,13$ ad haec margo $\sigma \tilde{\eta} \quad 15 \varepsilon v] \nu$ initio versus, omisso $\varepsilon$, quod rubro ascribendum erat 21 o $\left.\Gamma^{\prime} \alpha i ̈ 0 s\right]$ róïos. $\alpha \dot{v} \tau \tilde{\omega} v] \alpha \dot{v} \tilde{\imath}$ i. e. $\alpha \dot{v} \tau \dot{v} v$.

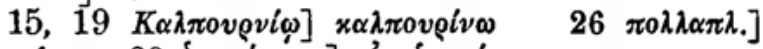

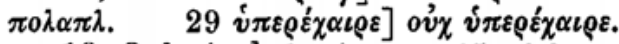

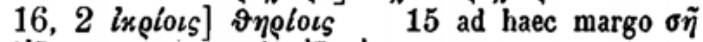

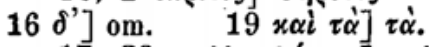

17, $\left.23 \pi 0 \lambda \lambda \alpha \pi \lambda \alpha_{\alpha}^{\prime} \sigma \iota \nu\right] \pi 0 \lambda \alpha \pi \lambda \alpha \dot{\sigma} \iota \nu$.

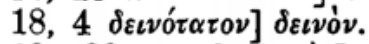

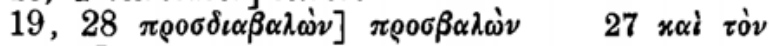
$K \alpha ́ \lambda \lambda \iota \sigma \tau 0 \nu]$ om.

20,6 i $\left.\pi \omega \dot{\pi} \pi \varepsilon v \varepsilon \sigma \varphi \tilde{\alpha}_{S}\right]$ ita cod., non $\sigma \varphi \tilde{\alpha}_{S}$ i $\pi \omega \dot{\pi} \tau \varepsilon v \varepsilon$, ut perhibet Pinderus, idem ex Vindob. afferens.

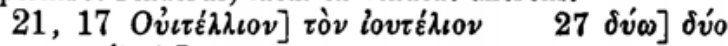
$\left.30 \pi \varrho 0 x \alpha \tau \varepsilon^{\prime} \lambda \alpha \beta \varepsilon\right]$ om. 
22,12 ó] $\left.\omega_{\mathrm{S}} \quad 17 \dot{\varepsilon} \xi \varepsilon \mu \alpha i v \varepsilon \tau 0\right]$ om.

23,12 sequentibus superscr. rubro col.: $+\alpha \dot{\alpha} \alpha \varrho \varrho \eta \sigma \iota s$

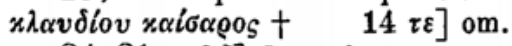

24, $21 \tau \eta \vartheta \eta \overline{]}$ Ita cod. quoque cum Wolfio et Duc., quod tamen rectius scribi videtur ut Pinderus tacito $\tau \eta^{\prime} \vartheta \eta$.

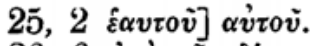

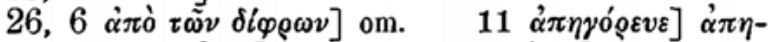

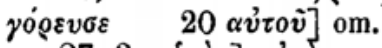

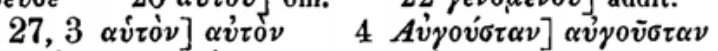

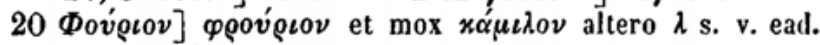
m., duplici infra.

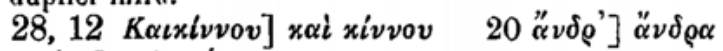
$\chi \alpha \lambda \varepsilon \pi \alpha i \nu \eta] \chi \alpha \lambda \varepsilon \pi \eta \dot{v} v$.

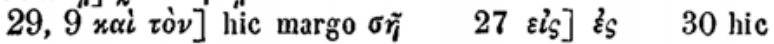
margo $\sigma \tilde{\eta}$ ut.

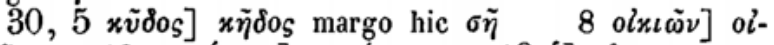

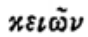

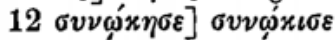

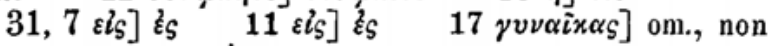
ut Pinderus dicit, $x \alpha i \gamma$.

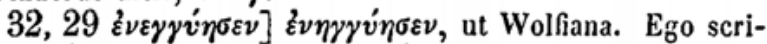
psi $\varepsilon v \varepsilon \gamma u ́ \eta \sigma \varepsilon v$, quod ad Parisinae $\varepsilon v \varepsilon \gamma \gamma v ́ \eta \sigma \varepsilon v$, a Pindero servatum, taceret Haasius, qui idem $\varepsilon^{2} v \varepsilon v \dot{\eta} \sigma \varepsilon v$ ex Parisino annotavit p. 537, B. Atque haec forma convenit passivae $\varepsilon \gamma \gamma \varepsilon \gamma v \eta$ -

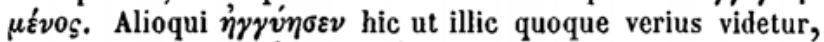

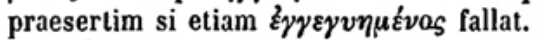

33, $\left.11 \sum 0 \sigma i \beta \iota \nu\right] \sigma \omega \sigma i \beta \iota \nu \nu 19$ of $\left.\alpha v i o i_{S}\right]$ of s. v.

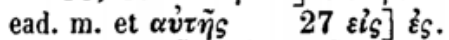

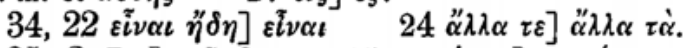

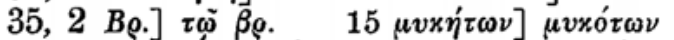

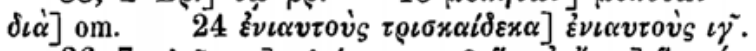

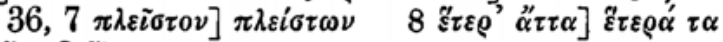
15 " $\lambda \eta \nu]$ ¿ौ $\lambda \eta \nu$.

$37,17$ ó $\pi \alpha \delta \dot{o} \nu]$ ó $\pi \alpha \delta \dot{\nu} \nu$ sequentibus rubro su-

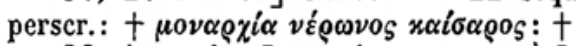

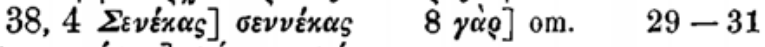

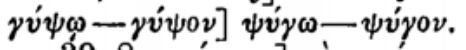

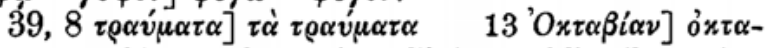
ovia ut $\mathbf{A}$ hic et 16, quod ex libris vix fallentibus etiam 
paullo ante fuisse restituendum colligitur, quum sit etiam apud Dionem, quem in his sequitur Zonaras $\mu \varepsilon \tau \alpha x \lambda \eta \hat{v} \tilde{\eta} \quad x \alpha i \mu o \imath \chi \varepsilon i \alpha \varsigma]$ om. $21 \alpha \dot{v} \tau \dot{\eta}] \alpha \dot{v} \tau \dot{\eta}$.

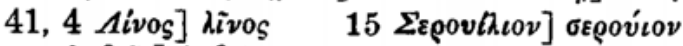
$19 \pi \rho 0 \sigma \lambda \alpha \beta \varepsilon i \nu] \lambda \alpha \beta \varepsilon i \nu$.

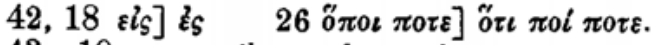

43,10 sequentibus rubro col. superscr.: $\mu$ ${ }^{2} \alpha \varrho \chi^{i \alpha} \alpha$

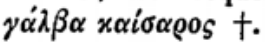

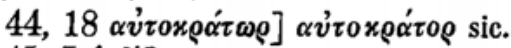

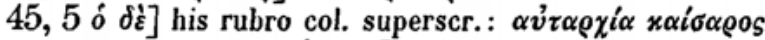

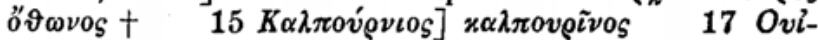

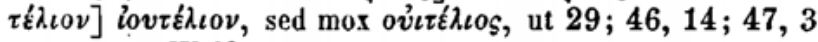
et seq. cum Wolfiana.

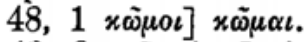

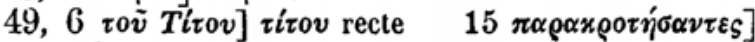
$\pi \alpha \rho \alpha x \rho \alpha \tau \dot{\eta} \sigma \alpha \nu \tau \varepsilon$.

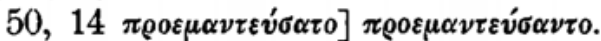

51, $3 \mu \dot{\varepsilon} v]$ om. 29 है $\varphi \eta]$ om.

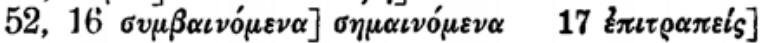
$\varepsilon \pi \iota \rho \varepsilon \iota \alpha_{S}$ in ras., sed ut nihil aliud appareat pr.

$\left.53,15 \mu \alpha^{\prime} \chi \alpha \iota \varsigma \tau \varepsilon\right] \mu \alpha^{\prime} \chi \alpha \iota \varsigma$.

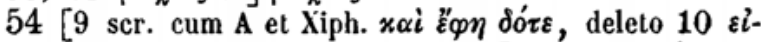

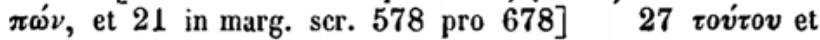

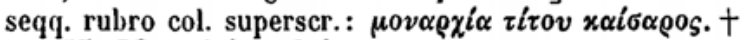

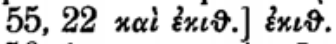

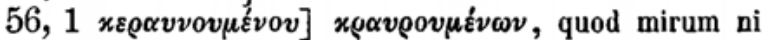
habeat etiam A cum Xiphilino, quamvis taceat collatio Haasii

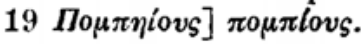

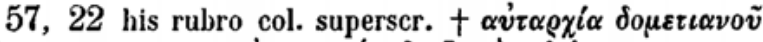

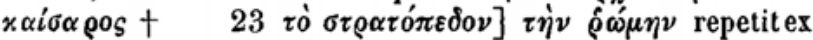
pracedent.

$58,12 \dot{\alpha} \delta \varepsilon \lambda \varphi \iota \delta \tilde{\eta}] \dot{\alpha} \delta \varepsilon \lambda \varphi \tilde{\eta}$.

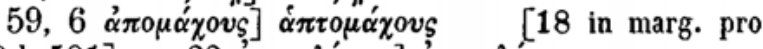

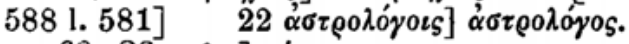

$60,26 \chi \varepsilon \hat{\imath} \varrho \varepsilon] \chi \varepsilon ́ \rho \varepsilon$.

$61,\left[10\right.$ post $\Sigma \tau \varepsilon \dot{\varepsilon} \varphi \alpha \nu \varepsilon$ add. $\left.x_{\alpha} \alpha \omega_{s}, \Sigma \tau \varepsilon \dot{\varphi} \varphi \alpha v \varepsilon\right] \quad 21$ f'] om. 
$\left.62,22 \dot{\eta} \delta^{\prime} \dot{\eta} \gamma \varepsilon \mu 0 v i \alpha\right]$ his rubro col. superscriptum :

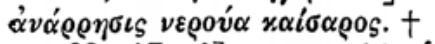

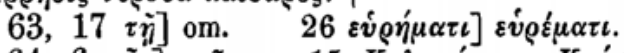

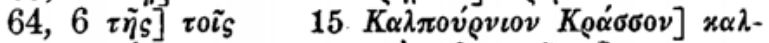

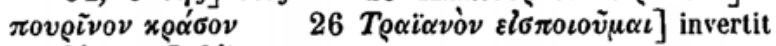

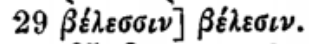

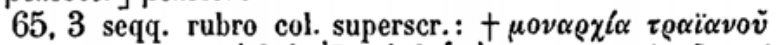

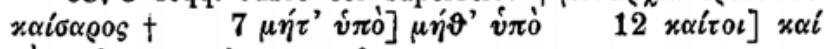
$\tau \dot{o}$, sed corr. ead. m., pr. fort. $\tau \iota$.

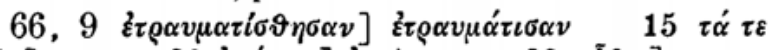

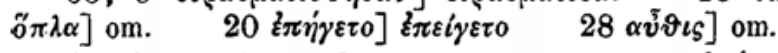

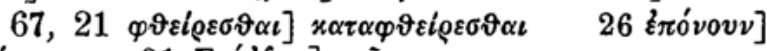

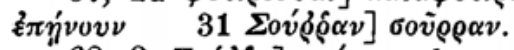

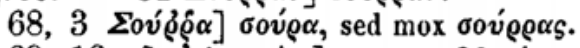

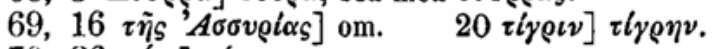

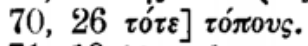

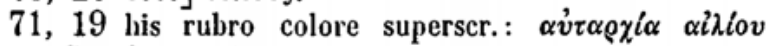

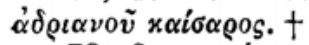

$\left.72,8 \sigma v \mu \mu \alpha \alpha_{0} 0 v \sigma l\right] \sigma v \mu \mu \alpha \chi i \sigma \iota \quad 18$ ó $\left.\tau \iota\right]$ om. 21

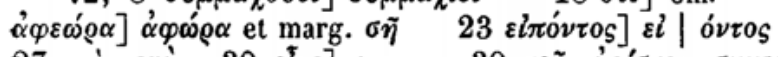

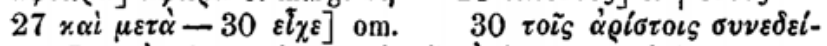

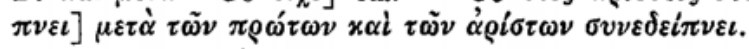

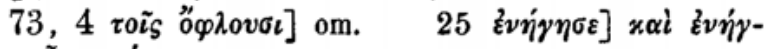

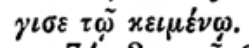

$74,3 \tau \iota \mu \tilde{\alpha} \sigma \vartheta \alpha \iota]$ in fine versus in marg. ead. $\mathrm{m}$.

16

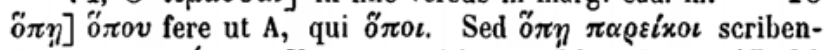
dum pro $\pi \alpha \rho \eta^{x}$. Ut. Utrumque vitium in libris Dionis 47, 36 $22 \dot{\alpha} \nu \delta \varrho \omega ั v \delta \dot{\varepsilon}] \dot{\alpha} \nu \delta \rho \tilde{\nu} v$.

76,30 "Avvıvov] $v$ alterum s. v. ead. m. Bĭৎov

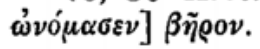

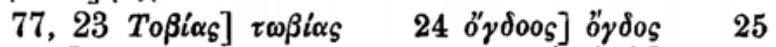
$\left.\Sigma \varepsilon v \varepsilon x \tilde{\alpha}_{S}\right]$ alterum $v$ s. v. ead. m. $16 \Lambda \varepsilon v i \varsigma \delta \omega \delta$.] om.

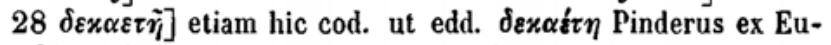
sebio tacito, ut praecipiunt grammatici.

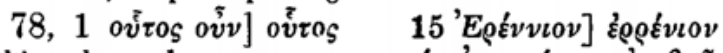
20 his rubro col. superscr. $\mu 0 v \alpha \varrho \chi i \alpha \alpha \nu \tau \omega v i \nu 0 v \varepsilon v \dot{v} \sigma \beta \beta_{0} \tilde{v}_{S}$ 25 o 'Avr.] om. 
LXXII

79, $7 \dot{\varepsilon} \xi \eta \tau.] \quad \dot{\xi} \xi \eta \tau$.

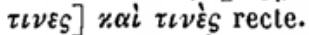

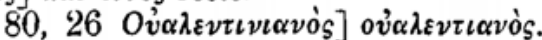

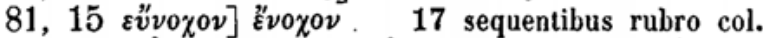

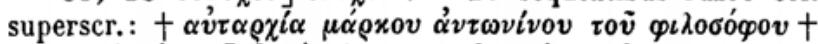

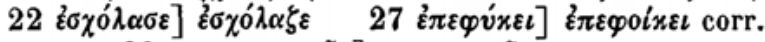
ead. $\mathrm{m}$. $28 \sigma \tau \rho \alpha \tau \iota \omega \tau \iota x \omega \tilde{\omega})] \sigma \tau \rho \alpha \tau \iota \omega \tau \tilde{\omega} \nu$.

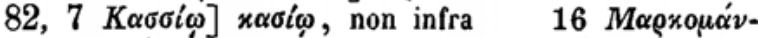
$v 0 \iota \varsigma] \mu \alpha \rho x o \mu \alpha \alpha^{\prime} \iota_{S}$ hic et $\left.3120 \gamma v v \alpha \iota x \tilde{\omega} \nu\right] \nu \varepsilon x \rho \tilde{\omega} \nu$ pr., corr. ead. $m$.

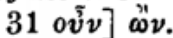

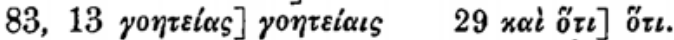

$84,25 \pi \alpha \varrho \alpha \sigma x \varepsilon v \kappa \alpha \zeta o \mu \varepsilon \dot{\varepsilon} \omega]-\nu \omega \nu \quad 26 \dot{v} \mu \tilde{\omega} \nu]$ om.

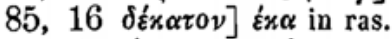

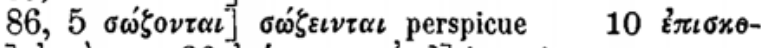

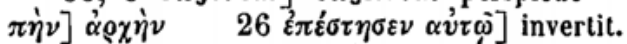

87,26 हैंน $\left.\mu \tilde{\alpha} \lambda \lambda_{0} \nu\right]$ ह่ $\pi \iota \mu \tilde{\alpha} \lambda \lambda_{0} \nu$.

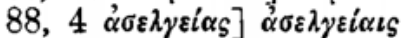

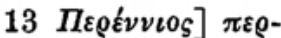

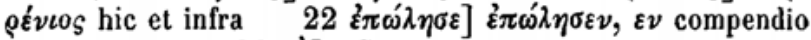
superscripto $30 \tau \dot{0}] \tau \tilde{\omega}$.

89, $3 \delta \iota \alpha \varphi \vartheta \varepsilon i \varrho \alpha \sigma \iota \nu]^{\prime} \delta \iota \alpha \varphi \vartheta \varepsilon i \varrho \omega \sigma \iota \nu \quad 5 \tau \omega \nu \delta^{\prime} i \pi \pi \omega \nu$

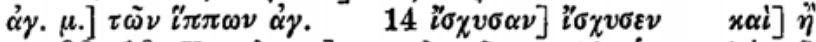

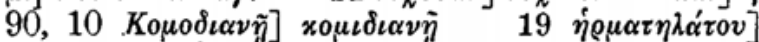

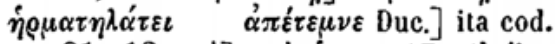

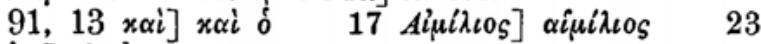
ws $\dot{\alpha} \lambda.] \varepsilon i s \alpha \lambda$.

$92,9 \delta \dot{\varepsilon} x \alpha i] \delta \dot{\varepsilon} \quad 15$ rò $\tau \tilde{\omega} \quad 24$ seqq. rubro col. superscr. $+\mu 0 \nu \alpha \varrho \chi^{\prime} \alpha \pi \varepsilon \rho \tau i \nu \alpha$ s..+

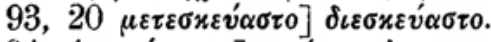

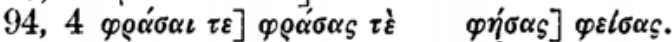

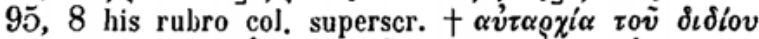

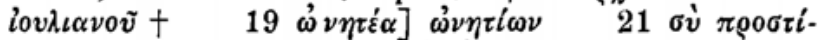

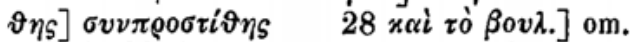

$96,23 \varepsilon^{2} v$ s. v. ead. m. $\left.25 \delta \varepsilon\right]$ om.

$97,15 \delta]$ non om., ut perhibet Pind., qui alium fortasse dicere voluerat codicem $\left.\left.28 \delta^{\prime}\right] \delta \dot{\varepsilon} \quad 32 M \varepsilon \sigma \sigma \alpha^{\prime} \lambda \alpha\right] \mu \varepsilon^{\prime}-$ $\sigma \alpha \lambda \alpha$ sic.

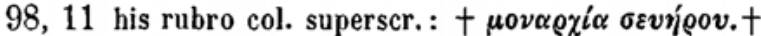

$99,7 \not \eta \vartheta \varepsilon \sigma \iota \nu] \not ̋ \vartheta \varepsilon \sigma \iota \nu$, ut ego, etsi de A nihil notatum 


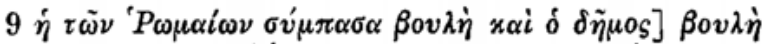
- $\delta \tilde{\eta}_{\mu \circ}$ om., ut A: $\delta \dot{v} v \alpha \mu \iota \varsigma$ pro his Xiphilinus 74,3 . Sed Dio si omisit substantivum ad quod refertur $\dot{\eta} \sigma v \dot{\mu} \mu \alpha \sigma \alpha$, id

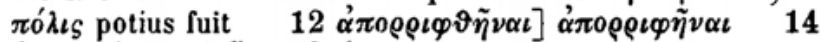

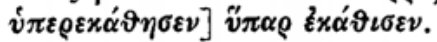

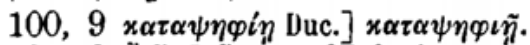

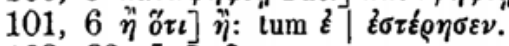

$\left.102,23 \varepsilon^{i} \chi^{\varepsilon}\right] \varepsilon \chi^{i} \varepsilon v$ om., superscripto super $\chi$ compendio quo hoc potius quam $\varepsilon$ exprimitur $28 \tau \alpha \tilde{v} \tau \alpha] \tau \alpha \dot{v} \tau \eta$ S.

$103,19 \lambda \dot{\varepsilon} \varepsilon \varepsilon \iota x \alpha i] \lambda \dot{\varepsilon} \gamma \varepsilon \iota \dot{\varepsilon}$.

$\left.104,5 \not \ddot{\alpha} \nu \rho \alpha_{S}\right] \alpha_{\mathcal{S}}$ erasum: pr. fortasse fuerat etiam post hoc $x \alpha i \quad 11 \Pi \lambda \alpha v \tau L \alpha \nu 0 \tilde{v}] \pi \lambda \alpha v \tau i v o v$ hic, non infra $21 \Phi \tilde{\eta} \lambda \iota \xi] \varphi i \lambda \eta \xi \quad 27 \lambda 0 \chi \tilde{\eta} \sigma \alpha \iota] \lambda \sigma \gamma \chi \tilde{\eta} \sigma \alpha \iota \quad 30 \lambda 0 \chi \omega \dot{\delta} \eta]$ $\lambda 0 \gamma \gamma \omega \dot{\delta} \eta$.

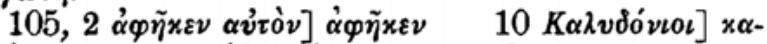

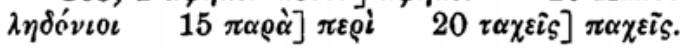

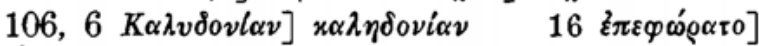

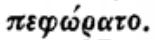

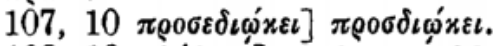

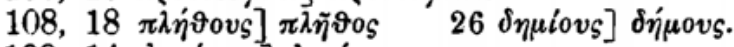

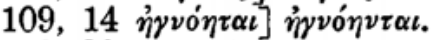

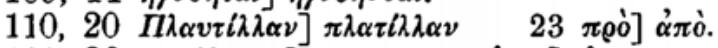

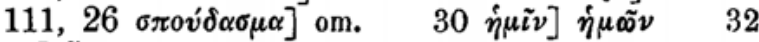
है $\chi 0 \mu \varepsilon \nu] " z \chi \omega \mu \varepsilon \nu$.

$\left.112,15 \mu \dot{\varepsilon} v \tau o l] \mu \dot{\varepsilon} \nu \quad 23 \sigma \tau \varepsilon \tilde{\chi}_{\chi}^{\varepsilon}\right] \sigma \tau o i \chi^{\varepsilon} \quad 30 \dot{\alpha}^{2} \lambda \lambda \dot{\alpha}$

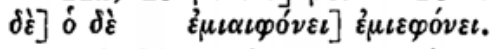

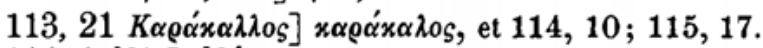

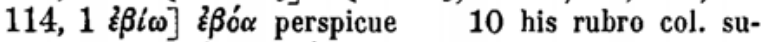

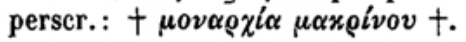

115, $12 \mu \alpha \nu \tau \varepsilon l \omega \nu$ Duc., quem tacito sequutus est Pind., $\mu \alpha \nu \tau \varepsilon \iota \omega \tilde{\nu}$ Wolfius et cod., quod quum haud dubie sit etiam in $\mathrm{A}$, de quo tacet Haasii collatio, revocandum $22 x \alpha i$

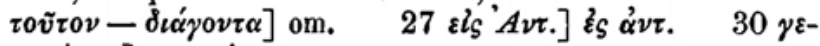

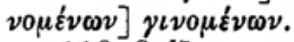

$116,8 \delta$ ] om. 17 seqq. rubro col. superscr. $+\dot{\alpha} \beta i-$

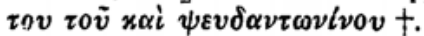

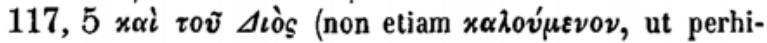




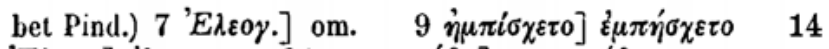

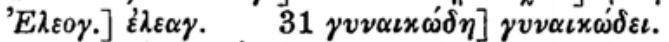

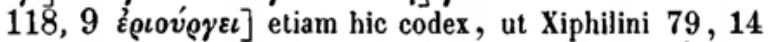

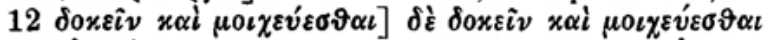

$\left.16 \delta^{\prime}\right] \delta \varepsilon \dot{~ u t ~ W o l f i u s ~ e t ~ h a u d ~ d u b i e ~ A, ~ p r o ~ q u o ~} \delta$ 'Duc.,

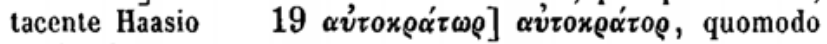
scribendum.

$\left.119,9 \mu \varepsilon \tau^{\prime}\right]$ ovं $\mu \varepsilon \tau$ ', ut A et Vindob., quo de miro pleonasmo dixi ad Stephani Thes. v. $\mu \varepsilon \tau \dot{\alpha}$, p. 841, A. Qui apud veteres ex ovं $\mu \varepsilon \tau \dot{\alpha}$ et $\mu \varepsilon \tau$ ' ov inter se permutatis,' ut apud Iosephum A. 1. 1, 22, 1, ortus, vix apud recentiores ferendus

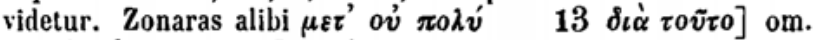

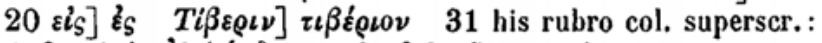

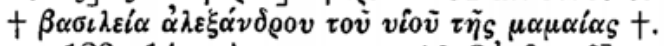

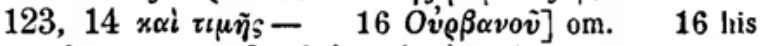
rubro col. superser.: $\beta \alpha \sigma \iota \lambda \varepsilon i \alpha \mu \alpha \xi \iota \mu i v o v+$.

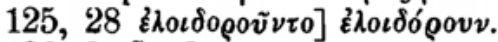

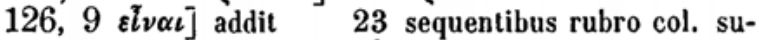

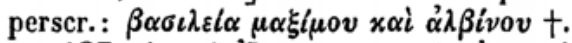

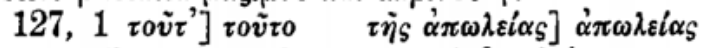

12 sequentibus rubro col. superscr.: $† \beta \alpha \sigma \iota \lambda \varepsilon i \alpha \pi 0 \mu \pi \iota \alpha \nu 0 \tilde{v} \dagger$ $\Pi о \mu \pi \eta \iota \alpha \nu o ́ v] \pi 0 \mu \pi \iota \alpha \nu o ́ v 13$ sequentibus rubro col. su-

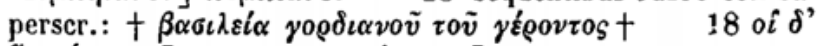

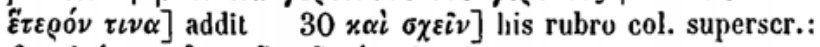
$\beta \alpha \sigma \iota \lambda \varepsilon i \alpha$

128, $\left.6 \dot{\alpha} \pi \alpha^{\prime} \gamma \xi \varepsilon \sigma \vartheta \alpha \iota\right] \dot{\alpha} \pi \alpha \gamma \xi \alpha \sigma \vartheta \alpha \iota \quad 10 \pi \rho \varepsilon \sigma \beta v \tau \varepsilon \dot{\rho}(0 v]$ $\pi \varrho \varepsilon \sigma \beta v^{\prime} 0 v$.

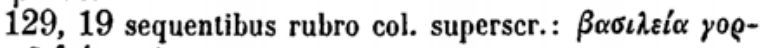

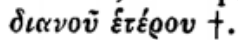

130,13 sequentibus rubro col. superscr.: $+\beta \alpha \sigma \iota \lambda \varepsilon l \alpha$

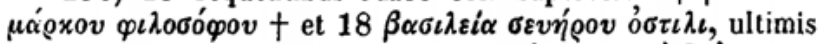

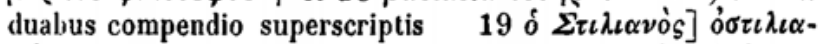

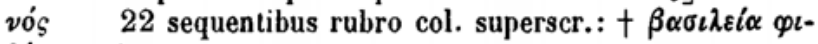
$\lambda i \pi \pi 0 v+$.

$131,4 \delta \dot{\varepsilon}] \delta$ ' ut Wolfius et haud dubie A 19 ov́$\tau \omega s$ Duc.] ovंos of $\Phi$.] $\varphi$.

$[133,4$ add. annot. $\pi \rho 0 \varepsilon i \varrho \eta \tau \alpha \iota]$ p. 612, A] $8 \mu \varepsilon$ $\left.\tau \eta \lambda \lambda.] \mu \varepsilon \tau \alpha \lambda \lambda . \quad 21 \tau 0 i_{S}\right] \tau \tilde{\eta} s$. 
134, 20 ' $\nu$ ' $\delta-\varepsilon x \varepsilon i v o v]$ om.

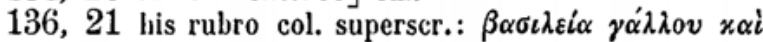

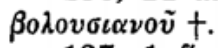

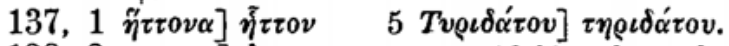

138, 8 $\psi \eta \varphi \iota \sigma$.$] ह่ \pi \iota \psi \eta \varphi \iota \sigma$. recte 10 his rubro col. su-

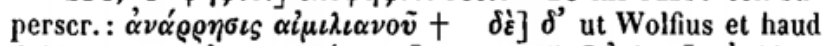

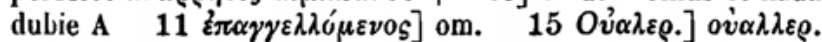
hic et infra, ubi $\lambda$ Ducangius, qui hic $\lambda \lambda$, utrumque ut Wolfius $24 \mu \dot{\nu} \nu] \mathrm{om}$.

139, 20 his rubro col. superscr.: $\beta \alpha \sigma \iota \lambda \varepsilon i \alpha$ ov $\alpha \lambda \lambda \varepsilon \rho \iota \alpha-$ vov $x \alpha i \gamma \alpha \lambda \eta i v o v+$ $28 \Theta \rho \alpha x \omega ́ \alpha \nu] \stackrel{\alpha}{\alpha \vartheta \rho \alpha x \omega ́ \alpha \nu . ~}$

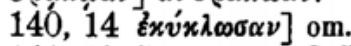

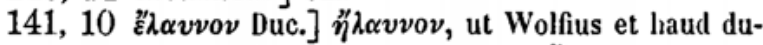

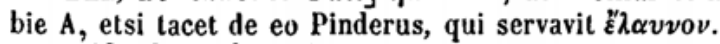

142, 26 Ovं $\alpha \lambda \varepsilon.] \beta \alpha \lambda \lambda \varepsilon \rho$.

143, 1 his rubro col. superscr. $\beta \alpha \sigma \iota \lambda \varepsilon i \alpha$ $\gamma \alpha \lambda \eta i v o v+$ $\left.4 \begin{array}{lll}\varepsilon & \tau & \tilde{\eta}\end{array}\right] \tau \tilde{\eta}$, ut Wolfius. Itaque nullius fidei videtur illud etiam ad sententiam incommodum $\dot{\varepsilon} \nu$, etsi tacet de A Haasius,

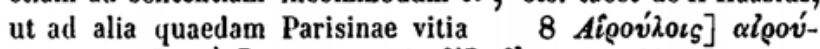

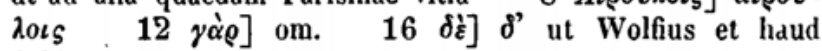
dubie A.

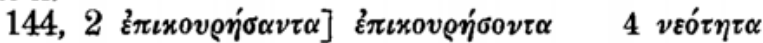

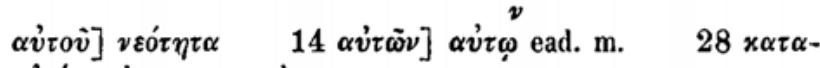
$x \lambda \varepsilon i \sigma \alpha_{S}$ in marg. ead. $\mathrm{m}$.

$\left[146,22\right.$ excidit infra ${ }^{\circ}$ littera 0.$]$

147, $13 \tau \iota \nu \alpha \varsigma] \tau \iota \nu \omega \nu \quad 29 \dot{\alpha} \nu \varepsilon \lambda \varepsilon \tilde{\imath} \nu \alpha \dot{v} \tau \dot{\nu} \nu]$ om.

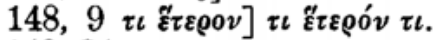

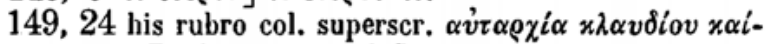

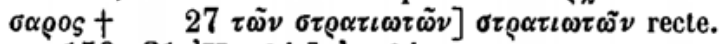

$150,21 ' H \mu \alpha \vartheta i \alpha] \dot{\alpha} \mu \alpha \vartheta i \alpha$.

151, 23 $\alpha v \tau \alpha \rho \chi i \alpha v] \beta \alpha \sigma \iota \lambda \varepsilon i \alpha \nu$, ut A, sed margo ead. m.

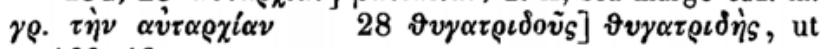
p. $160,12$.

152,3 sequentibus rubro col. superscr.: $\beta \alpha \sigma \iota \lambda \varepsilon l \alpha \alpha \dot{v}$ $\varrho \eta \lambda \iota \alpha \nu 0 \tilde{v}+\alpha \dot{v} \tau o \tilde{v}]$ om.

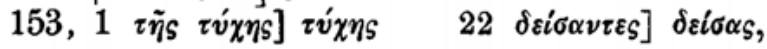


compendio scripta ultima 26 his rubro col. superscr.:

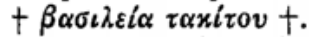

$\left.154,13 \varepsilon \pi \iota \delta \iota \omega^{\prime} \alpha \nu \tau \varepsilon \xi\right]$ om. 16 his rubro col. su-

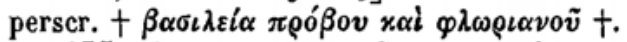
$\tau \iota \tilde{\omega} \tau \iota$.

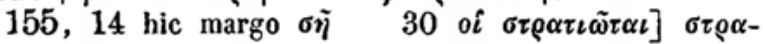

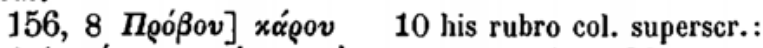

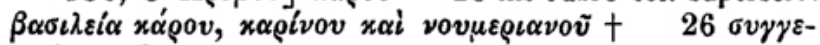

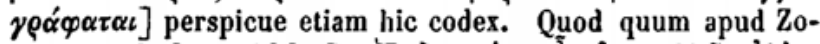

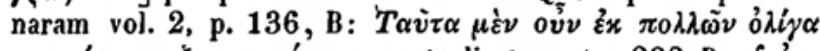

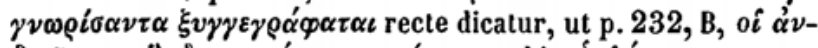

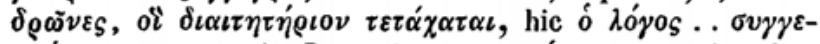

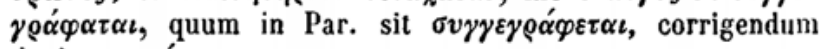

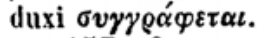

157, 6 sequentibus rubro col. superscr.: $+\alpha \dot{v} \tau \alpha \varrho \chi^{i \alpha}$

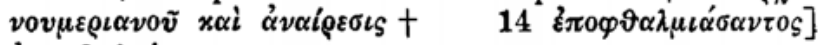
$\varepsilon \pi 0 \varphi \vartheta \alpha \lambda \mu i \sigma \alpha \nu \tau 0$ s.

$\left.\left.158,10 \tau \tilde{\eta}_{S} \tau \tilde{\omega} \nu\right] \tau \tilde{\eta}_{S} \quad 22 Z \dot{\alpha} \beta \delta \alpha_{S}\right] \xi_{\alpha}^{\prime} \mu \delta \alpha_{S} \quad 24$

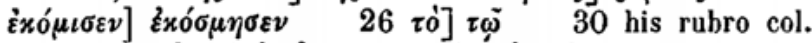
superscr. $+\beta \alpha \sigma \iota \lambda \varepsilon l \alpha \delta \iota 0 x \lambda \eta \tau \iota \alpha \nu 0 \tilde{v} x \alpha i \mu \alpha \xi \iota \mu \iota \alpha \nu 0 \tilde{v}+$.

159, $\left.20 \dot{\alpha} \pi \alpha^{\prime} \nu \tau \omega \nu\right]$ om. $\left.\quad 21 \mu \dot{\alpha} \lambda \lambda_{0 \nu} \delta \dot{\varepsilon}\right] \mu \dot{\alpha} \lambda \lambda_{0} \nu$.

$160,12 \vartheta v \gamma \alpha \tau \varrho \iota \delta \circ \tilde{v} v] \vartheta v \gamma \alpha \tau \rho \iota \delta \dot{\eta} v$, ut p. 151, 28

26 'A $A \alpha \mu \alpha \nu \nu 0 v$ '] $v$ alterum s. v. ead. m., quum duplex sit continuo.

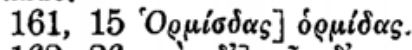

$\left.162,26 \tau 0 v_{S} \delta^{\prime}\right] \tau \tilde{\omega} \nu \delta^{\prime}$.

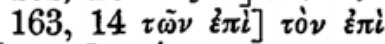
бxó $\mu \mu \alpha \tau \alpha] \sigma x \omega ́ \mu \mu \alpha \tau \alpha$.

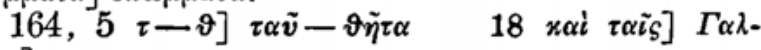
$\lambda i \alpha \iota s]$ om.

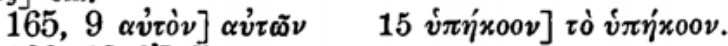

$\left.166,13 \delta^{\prime}\right] \delta \dot{\varepsilon}$ ut Wolfius et haud dubie A.

167, $2 \beta \alpha \sigma \iota \lambda \varepsilon i \alpha v]$ om. $\quad 5 \gamma \varepsilon \tau \omega \bar{\jmath}] \gamma \delta \dot{\varepsilon} \tau \omega$.

168,13 his rubro col. superscr.: $+\beta \alpha \sigma \iota \lambda \varepsilon i \alpha \mu \alpha \xi \iota \mu i \nu 0 v \dagger$ $15 \sigma \varepsilon i \varrho \alpha \nu] \sigma \varepsilon \iota \rho \dot{\alpha} \nu$ ut ego.

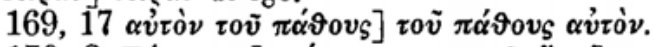

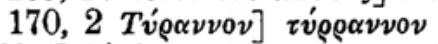

$\left.Z \alpha^{\prime} \beta \delta \alpha \nu\right] \zeta^{\prime} \alpha \dot{\mu} \delta \nu \nu$, ut supra p. 158, 22

3 हैros] om. 6 15 'Ivvox.] ivox., 
sed 17 ivvox.

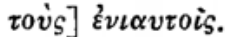

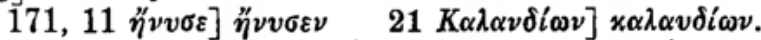

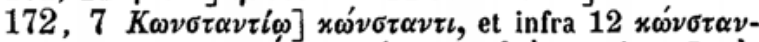

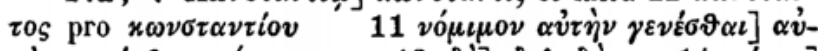

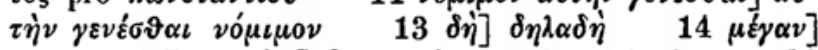
om. $\left.15 B \rho \varepsilon \tau \alpha v i \alpha_{S}\right] \beta \rho \varepsilon \tau \tau \alpha v i \alpha_{S}$, quod recipiendum 20

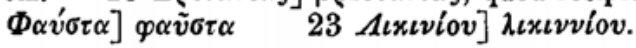

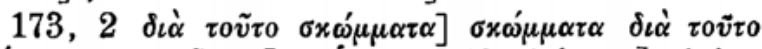
vंó $\tau \iota v \omega \nu \pi \varepsilon \pi 0 \iota \tilde{\eta} \sigma \vartheta \alpha \iota] \pi$. v. $\tau$. $13 \pi \lambda \varepsilon i \sigma \tau o v s] \pi \lambda \varepsilon i o v S$

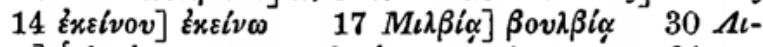

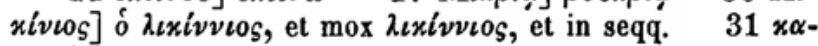
$\tau \varepsilon \lambda \varepsilon i \varphi \vartheta \eta \sigma \alpha \nu] \varepsilon \iota$ et $\eta$ per dittographiam.

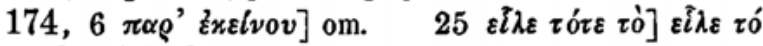
$\tau \varepsilon$, quod recipiendum.

175,3 addit $\tau \dot{o}$ post $\hat{\eta} \tau \iota \tilde{\omega} \nu \tau 0$ $\vartheta \bar{\eta} \nu \alpha \iota$ $9 \dot{\alpha} \nu \alpha \iota \rho \tilde{\eta} \sigma \alpha \iota] \dot{\alpha} \nu \alpha \iota \rho \varepsilon \vartheta \tilde{\eta} \nu \alpha \iota$

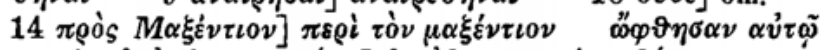

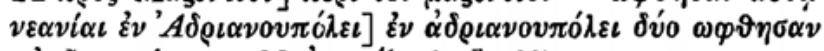

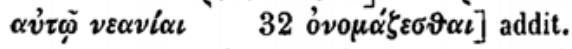

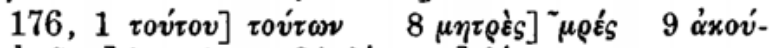

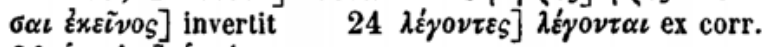

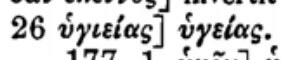

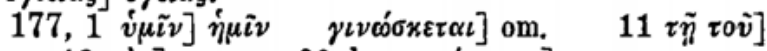

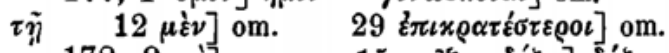

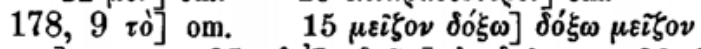

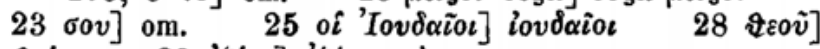

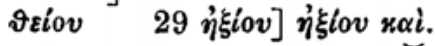

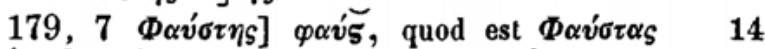
$\Phi \alpha \dot{v} \sigma \tau \alpha] \varphi \alpha \tilde{v} \sigma \tau \alpha$ hic et infra $22 \quad 15$ zxeivov bis, semel deleto altero, cuius erasum videtur prius $\varepsilon \quad 20 \delta i \dot{\alpha} \delta \dot{\varepsilon}]$

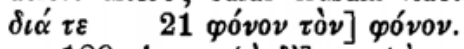

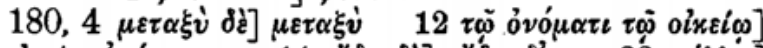

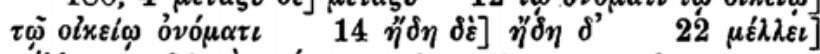

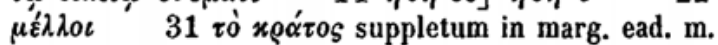

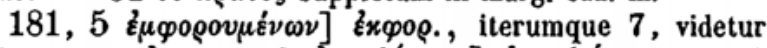

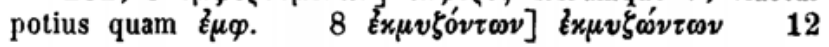




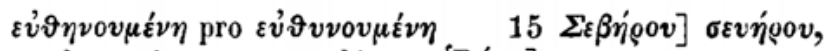

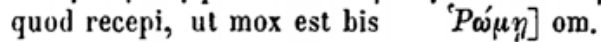

182, $8 \sigma \varphi \alpha \lambda \tilde{\omega} \sigma \iota] \sigma \varphi \alpha ́ \lambda \omega \sigma \iota$, sed pr. $\sigma \varphi \alpha \lambda \tilde{\omega} \sigma \iota$, quod scri-

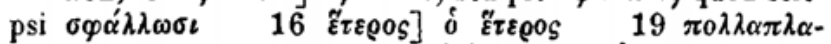

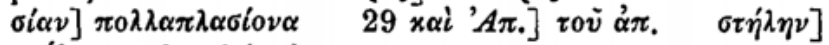
$\sigma \tau \eta^{\prime} \lambda \eta$, quod recipiendum.

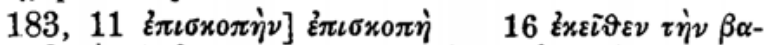

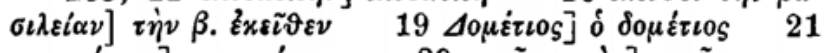

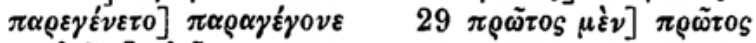

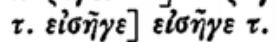

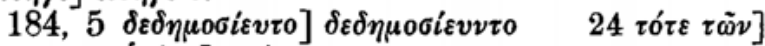
$\tau \tilde{\omega} \nu \quad 29 \dot{\alpha} \gamma i \omega \nu] \vartheta \varepsilon i \omega \nu$.

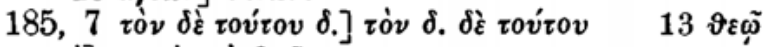

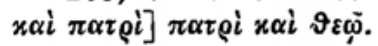

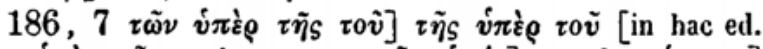

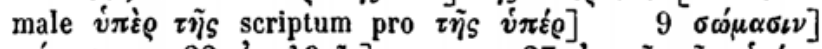

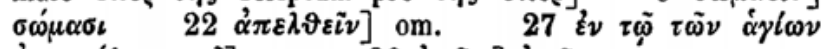

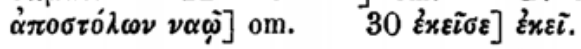

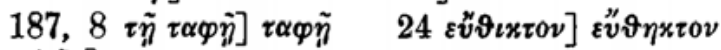
$30 \mu \varepsilon \lambda \hat{\omega} \nu] \mathrm{om}$.

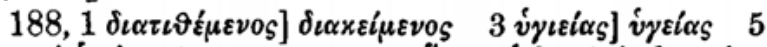
ante $x \alpha \dot{i}$ ó $\mu \dot{\varepsilon} \nu$ rubro col, superscr. †ó $\pi \omega s \hat{\eta} \beta \alpha \sigma i \lambda \varepsilon i \alpha$ $\oint \omega \mu \alpha i \omega \nu$

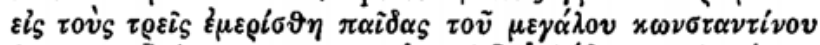

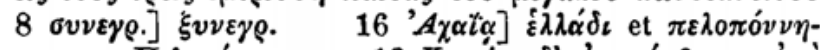

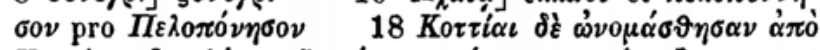

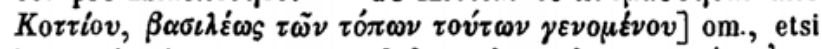

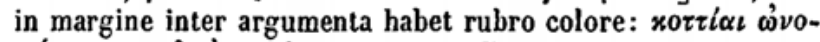

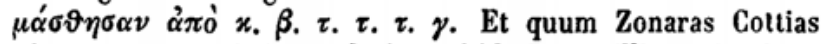
Alpes memoraverit iam vol. 1, p. 643, B, ut illic potius haec addenda fuissent, possunt sane haec ex margine illata videri, cui in codice, unde ductus est Monac., ascripta essent, ab librario autem inter argumenta relata esse quae desunt in textu, unde deest etiam ótı. Simile quid ad lib. 11, 10 inter argu-

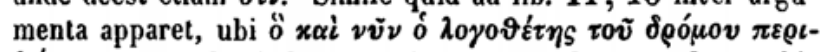
$\zeta \omega \dot{\nu} \nu v \tau \alpha \iota$, quod scholion est, inter cetera legitur. Itaque hic quoque illa ex margine illata videntur $20 \Pi$ ropvaiov]

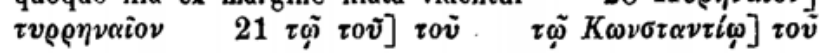




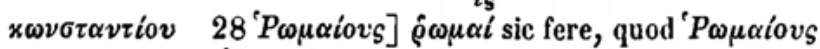

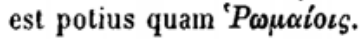

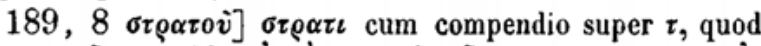

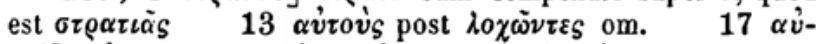

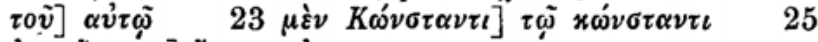

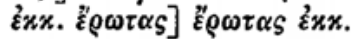

190, $3 \gamma v \nu \alpha \iota x \tilde{\omega} \nu], \gamma \alpha \mu \varepsilon \tau \tilde{\omega} \nu \quad 3 \tau \tilde{\omega} N \alpha \rho \sigma \tilde{\eta}] \nu \alpha \rho \sigma \tilde{\eta}$

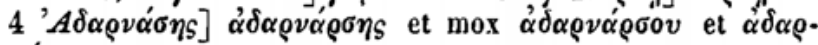
$\nu \alpha ́ \rho \sigma \eta \nu$.

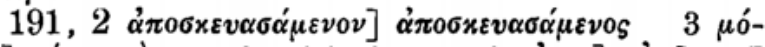

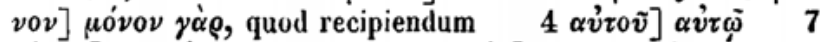

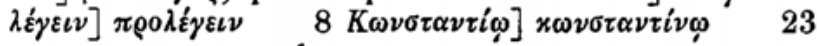

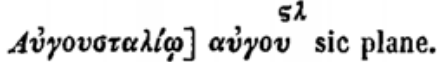

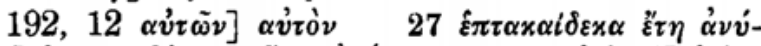

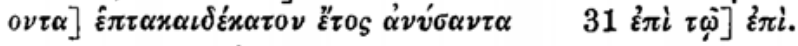

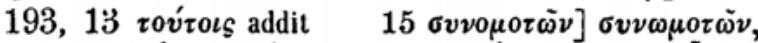
ut dedi $18 \varepsilon \dot{\varepsilon} \nu 00 v \mu \varepsilon \dot{\varepsilon} \omega \omega$ post $\left.\pi \varrho 0 \tau \iota \mu \eta \dot{\eta} \sigma \alpha \iota \tau \quad 26 \eta^{j} \lambda \vartheta \varepsilon\right]$ ที่ $\boldsymbol{\vartheta} 0 \nu$.

194, 1 jंv $\gamma \alpha \mu \beta \rho o \dot{s}] \gamma \alpha \mu \beta \rho o \dot{s} \eta^{5} \nu$ 12 ös] ôv 18

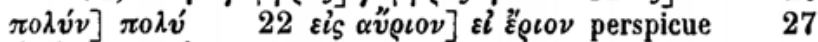

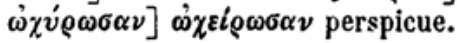

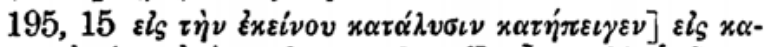

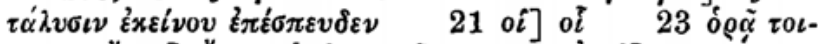

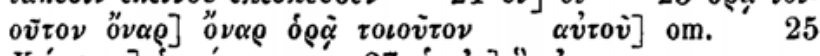

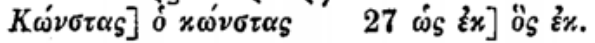

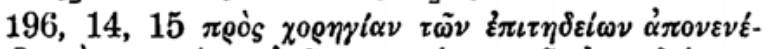

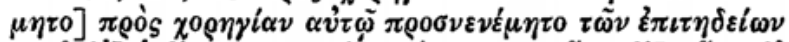

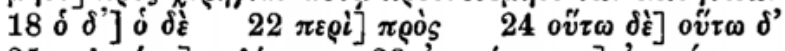

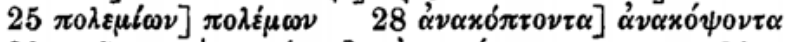

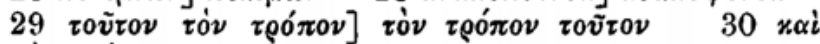
$\tau \dot{\eta} \nu] \tau \dot{\eta} v$.

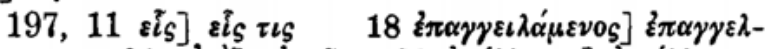

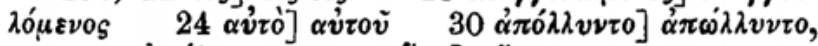

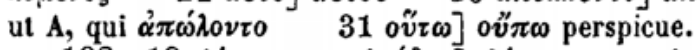

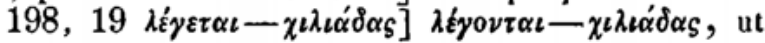




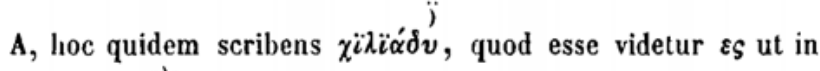
$\cdot$ ).

है $\gamma \chi \varepsilon \iota \varrho \iota 0 \nu \tau$ p. 199, 3. Nam $\chi \iota \lambda \iota \alpha ́ \delta^{\sim}$ p. 204, 1, cod. fol. 368 , est $\chi \iota \lambda \iota \alpha \delta \alpha S \quad 24 \pi \rho \varepsilon \sigma \beta \varepsilon v ́ \sigma \alpha \nu \tau \alpha] \pi \rho \varepsilon \sigma \beta \varepsilon v \dot{\sigma} \sigma o v \tau \alpha$

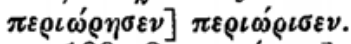

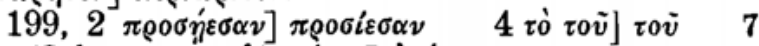

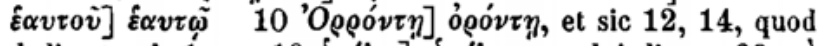
dedi, ut vol. $1 \quad 19 \delta \mu i \lambda \varepsilon \iota] \omega \mu i \lambda \varepsilon \iota$, quod dedi $20 \mu \dot{\gamma}$

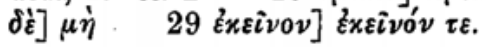

200, $3 \pi \varepsilon \rho \iota \lambda \iota \pi \tilde{\eta}] \pi \varepsilon \rho \iota \lambda_{0} \iota \pi \tilde{\eta} \quad 11 x \alpha i$ 甲ílovs] $\tau \varepsilon x \alpha i$

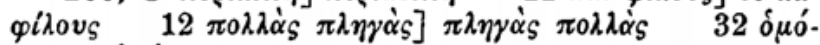

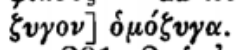

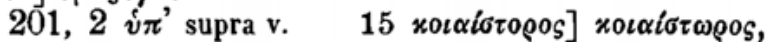

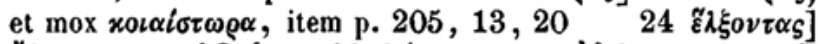

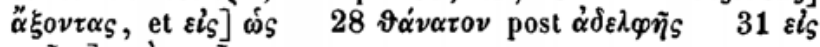

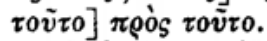

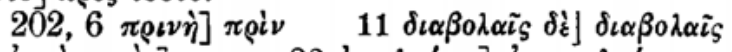

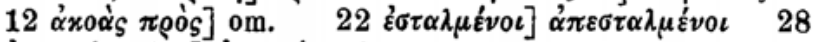

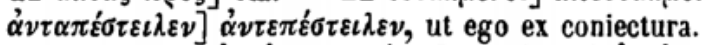

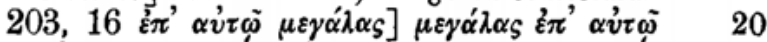

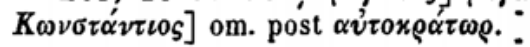

204, 3 'A $\left.A \alpha \mu \alpha \nu \nu o i_{\zeta}\right]$ alterum $\nu$ s. v. ead. m. $4 \eta \dot{v}$ -

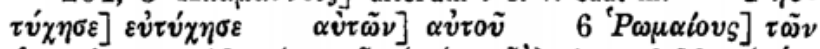

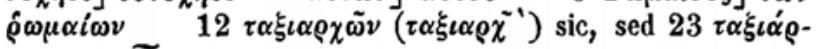
$\chi \omega \nu$ ( $\left.\tau \alpha \xi \iota \alpha \alpha^{\prime} \chi\right)$, quod bis dedi. Infra p. 214, $19 \tau \alpha \xi \iota \alpha \rho \chi$ sine

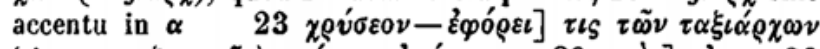

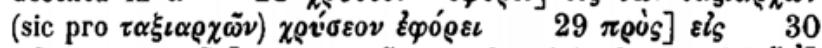
$\tau \tilde{\omega} \nu \sigma \tau \varrho \alpha \tau \iota \omega \tau \tilde{\omega} \nu] \sigma \tau \rho \alpha \tau \iota \omega \tau \tilde{\omega} \nu$, quod recipiendum $\left.31 i^{\prime \nu}{ }^{\prime}\right]$

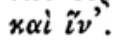

205, $1 \varepsilon i \varsigma$ $\dot{\omega} \varphi \varepsilon^{\prime} \varepsilon \iota \alpha \nu$ in margine ad finem versus ascri-

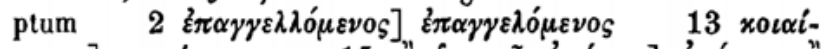

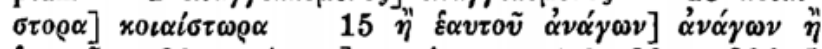

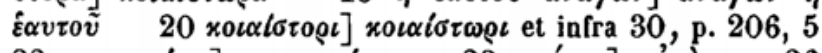

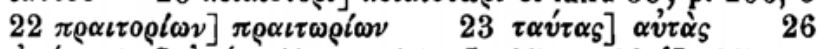
$\left.\alpha^{\prime} \pi \eta^{\prime} \gamma \gamma \varepsilon\llcorner\lambda \varepsilon \nu] \alpha^{\prime} \pi \eta^{\prime} \gamma \gamma \varepsilon \lambda \lambda \varepsilon \nu \quad 27 \sigma \varepsilon\right]$ addit $\left.32 \delta\right]$ addit.

$206,27 \xi \xi \circ \mu.] \quad \xi \xi \omega \mu . \quad 28 \xi \dot{v} \mu \pi \alpha \nu \tau \alpha \varsigma] \sigma \dot{v} \mu \pi \alpha \nu \tau \alpha_{S}$. $207,4 K \omega \nu \sigma \tau \alpha \nu \tau i o v] \tau o \tilde{v} \times \omega \nu \sigma \tau \alpha \nu \tau i o v \quad 6 \alpha v i \omega \nu]$ 


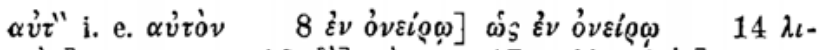

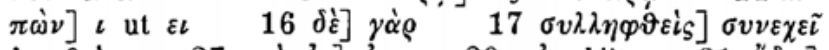

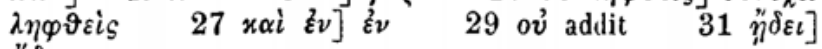
$\eta \not \delta \eta$.

208, $18 \pi \varepsilon \varrho i \tau \tau \varepsilon v \mu \alpha] \pi \varepsilon \rho i \tau \tau \omega \mu \alpha \quad \sigma v \nu \varepsilon \xi \varepsilon \rho \varrho v ́ \eta] \sigma v \nu$ -

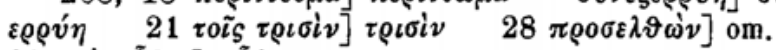
$\left.29 x \alpha i \alpha v^{3} \vartheta \iota \varsigma\right] \alpha v^{*} \vartheta \iota s$.

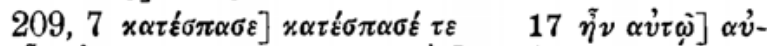

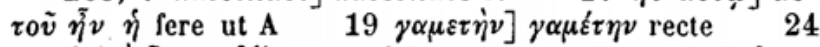
$\left.\pi \varepsilon \rho t \delta \varepsilon \xi \iota \iota_{\varsigma}\right] \pi \varepsilon \rho \iota \delta \varepsilon \xi_{\iota 0 S} 27$ superscr. rubro col. $+\beta \alpha \sigma \iota$ -

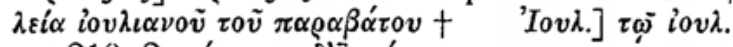

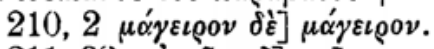

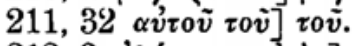

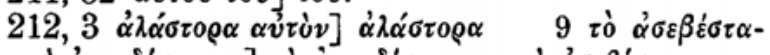

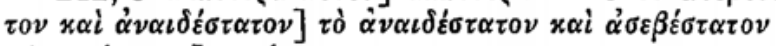
$14 \pi \varepsilon \rho i \varphi \eta \mu \rho \zeta] \pi \varepsilon \rho i \varphi \eta \mu \nu \nu$.

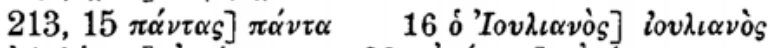

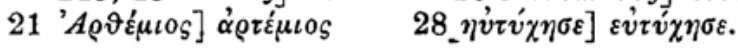

$\left.214,3 \pi \varepsilon \rho\llcorner\varphi \nu \tilde{\eta}] \pi \rho 0 \varphi \alpha v \tilde{\eta} \quad 4 \delta v^{\prime} \omega\right] \delta v_{0}$

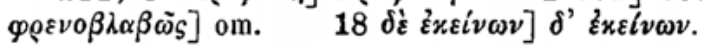

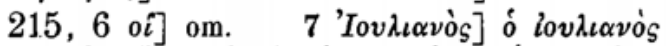

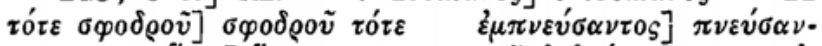

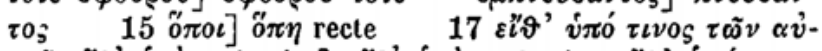

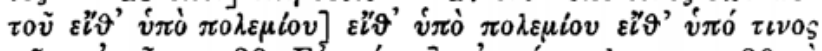

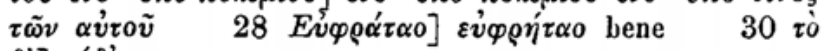
$\delta \grave{\varepsilon}] \tau \operatorname{có}^{\prime}$.

$216,6 \pi \varepsilon \rho i \delta \dot{\varepsilon}] \pi \varepsilon \rho i \quad 10 \xi \alpha \nu \vartheta \dot{o} \nu \tau \dot{\eta} \nu$ xó $\mu \eta \nu v \varepsilon \alpha-$

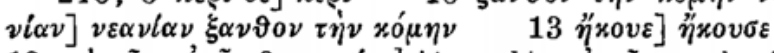
18 жi $\left.\tau \tilde{\eta}_{S} \alpha v \tau \tilde{\eta}_{S} \vartheta \rho \eta \sigma x \varepsilon i \alpha_{S}\right]$ bis, et bis $\alpha v \tau o \tilde{v}$, quod priori loco correctum in marg. al. $\mathrm{m}$. $26 \pi \rho \circ \mu v \eta \vartheta \varepsilon i \varsigma] \mu v \eta \vartheta \varepsilon i \varsigma$

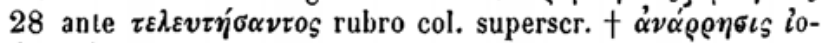
$\beta \iota \alpha \nu \circ \tilde{v}+$.

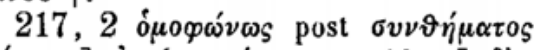

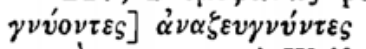
$\tau \alpha \rho \sigma j v$ recle, ut ed. Wolf.

$19 \tau \varepsilon] \delta \dot{\varepsilon}$

$12 \dot{\alpha} \nu \alpha \zeta \varepsilon v$.

218, 1 i $\pi \alpha \dot{\alpha} v \tau \eta \sigma \nu], i \pi \alpha \nu \tau \dot{\eta} \nu$ $21 \tau \dot{\eta} \nu$ ante" $A \gamma x v \varrho \alpha \nu]$ om.

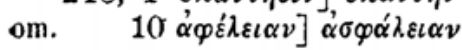
6 ż $\pi i$ ante $\omega \nu o ́ \mu \alpha \xi o v]$ zONATAS $\mathrm{V}$.

$\left.21 \alpha{ }^{\prime} \vartheta \varepsilon \delta_{\varepsilon}^{\prime}\right] \alpha \iota^{\prime} \vartheta \varepsilon$ 


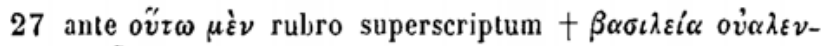
$\tau \iota \nu \iota \alpha \nu 0 \tilde{v}+$.

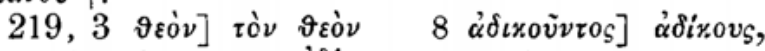
quod recipiendum, quum $\alpha \delta i \% o v$ notatum sit ex A.

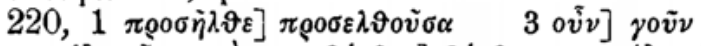

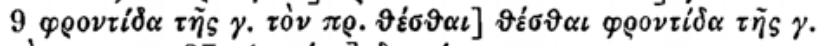

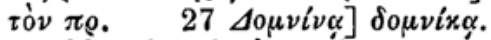

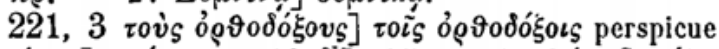
$\left.8 \tau o v i \omega \nu] \tau o v^{\prime} \sigma v 12 \delta^{\prime}\right]$ aldit $\left.16 \vartheta v^{\prime} \rho \alpha \iota\right] \pi v ́ \lambda \alpha \iota$

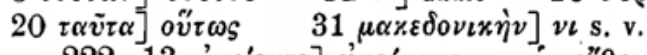

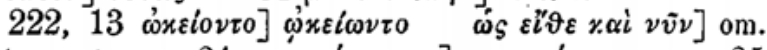

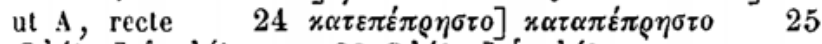

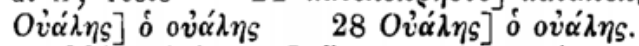

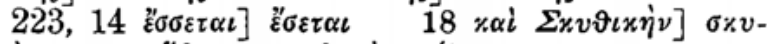

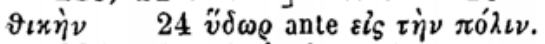

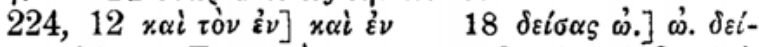
$\sigma \alpha_{S} \quad 26$ ante $\Gamma \varrho \alpha \tau \iota \alpha \nu o_{S}$ superscr. col. rubro $+\beta \alpha \sigma \iota \lambda \varepsilon i \alpha$

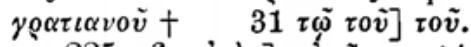

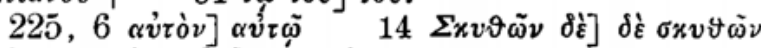

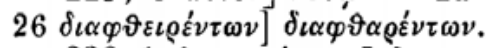

226, $1 \delta v \sigma \mu \alpha \chi o ́ \tau \alpha \tau 0 \nu] \delta v \sigma \mu \alpha \chi \omega ́ \tau \alpha \tau o \nu 15$ i்'] 17 post $\breve{\xi} \xi$ in fine pagr. rubro col. $\beta \alpha \sigma \iota \lambda \varepsilon i \alpha$ ov $\alpha \lambda \varepsilon \nu \tau \iota \nu \iota \alpha \nu$ v

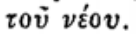

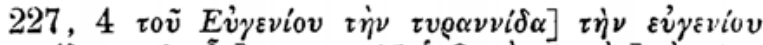

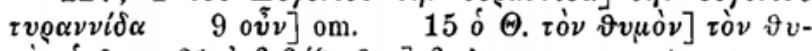
$\mu \hat{O} \nu$ o $\vartheta$. $23 \pi \rho \dot{s}]$ हis.

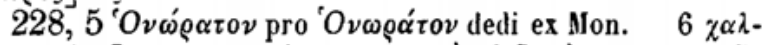

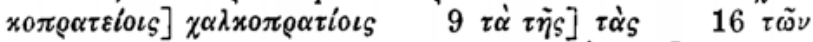
addidi ex eodem, ut est p. 227, $17 \quad 19 \pi 0 \iota \eta \sigma \alpha \nu \tau \alpha]$ om. 20

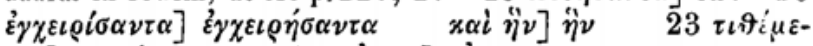

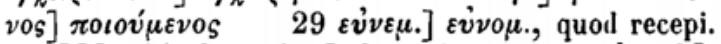

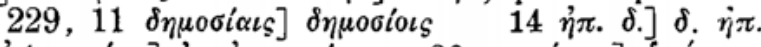

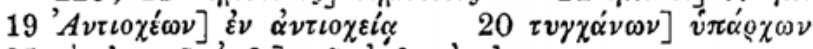

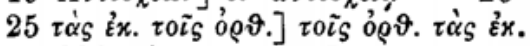

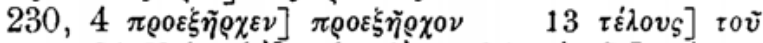

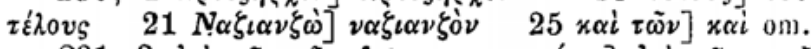

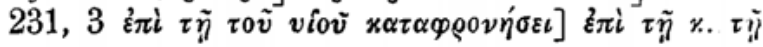




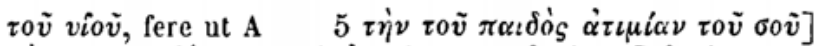

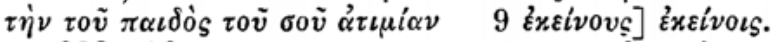

232,16 iis quae hic vulgo legebantur ov́rw $\delta\llcorner\alpha \nu \varepsilon \mu \eta$

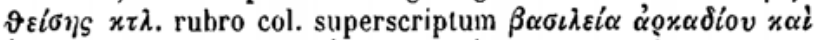

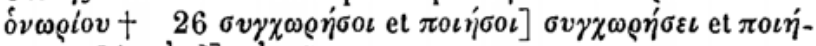

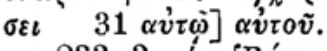

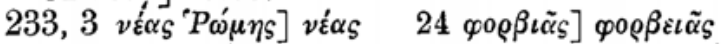

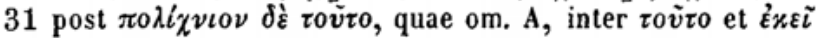
supra dimidium versus vacuum relictum, ut asteriscus est apud Wolfium post $\tau 0 \tilde{v} \tau o \quad 32 \delta$ v́o $\tau \varepsilon] \delta$ vío.

$234,15 \dot{\varepsilon} \pi \alpha \nu \dot{\eta} \lambda \vartheta \varepsilon \nu] \dot{\varepsilon} \pi \alpha \pi \hat{\eta} \lambda \vartheta \varepsilon \nu \quad 20 \hat{\varepsilon} \alpha v \tau 0 \tilde{v}] \alpha \dot{v} \tau 0 \tilde{v}$ $\left.22 \pi \alpha \iota \delta \iota x \tilde{\eta} \pi \alpha^{\prime} \nu v\right]$ invertit $\left.\quad 30 \tau \tilde{\omega} \nu\right] \tau \dot{o} \nu$.

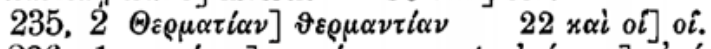

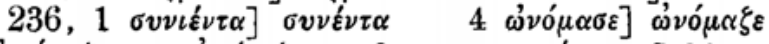
et $\alpha \nu \omega^{\prime} \mu \omega \xi \xi \nu$ pro $\alpha \nu \omega^{\prime} \mu \xi_{\xi} \varepsilon \nu$ et $8 \tau \iota \varsigma, \tau v \varrho \alpha \nu \nu \eta^{\prime} \alpha \varsigma$. Sed haec el. Duc. vitia: $\tau^{\dagger}$ (i. e. $\left.\tau \tilde{\eta}_{S}\right) \tau v \rho \alpha \nu \nu \eta^{\prime} \sigma \alpha$ Wolfius $24 \varepsilon \varepsilon_{S}$

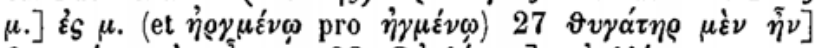

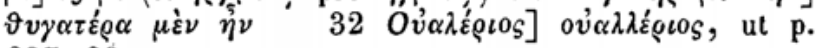
$237,29$.

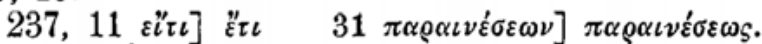

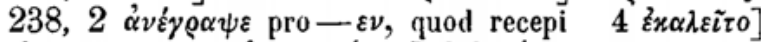

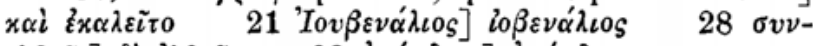

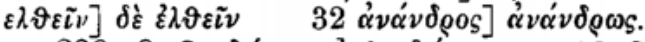

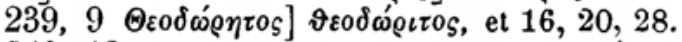

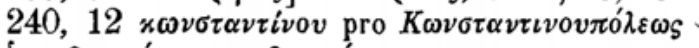

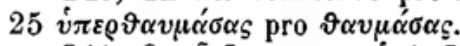

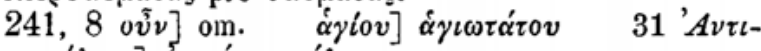

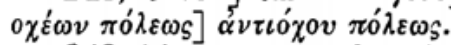

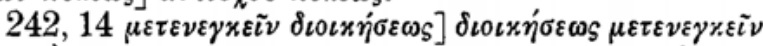

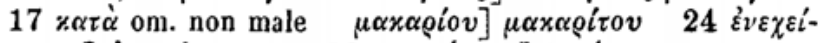

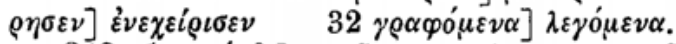

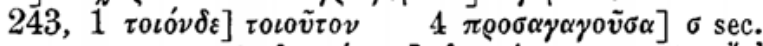

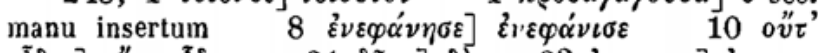

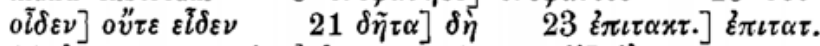

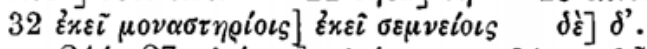

$244,27 \pi \lambda \varepsilon i \sigma \tau \omega] \pi \lambda \varepsilon i \sigma \tau 0 v \quad 31 \mu v \eta \vartheta \tilde{\eta} \nu \alpha \iota]$ om.

245,1 super $o ́ \mu \varepsilon \dot{v} \nu$ col. rubro superscr. $+\beta \alpha \sigma \iota \lambda \varepsilon^{\prime} \alpha \mu \alpha \varrho-$

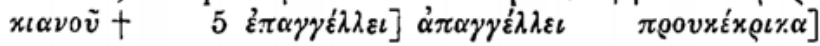


$\pi \varrho 0 x \varepsilon ́ x \varrho เ x \alpha \quad 12 \lambda \alpha \mu \pi \varrho \tilde{\omega} \nu] \lambda \alpha \mu \pi \varrho \circ \tilde{v} \quad 30 \alpha \dot{v} \tau \tilde{v} \nu] \alpha \dot{v}-$

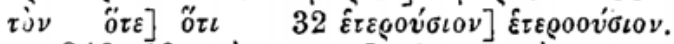

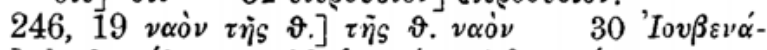

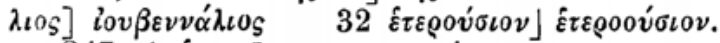

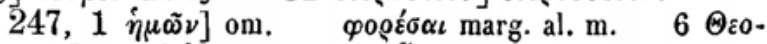

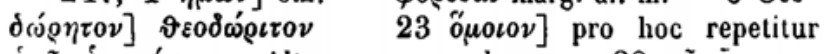

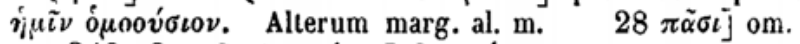

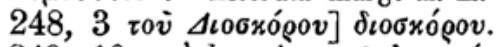

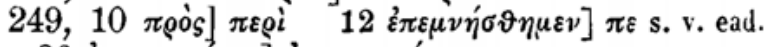

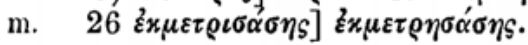

$\left.250,15 \varepsilon x x \alpha i \delta \varepsilon x \alpha] \quad \varepsilon \xi x \alpha i \delta \varepsilon x \alpha \quad 20 \tau \alpha^{\prime}\right]$ om. 23

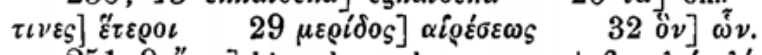

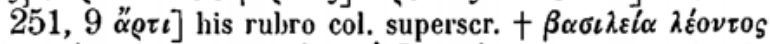

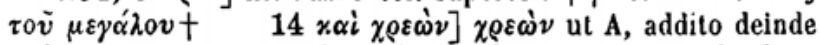

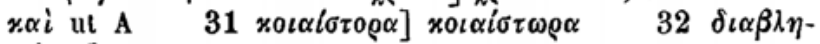
$\left.\vartheta{ }^{\prime} \nu \tau \alpha\right]$ om.

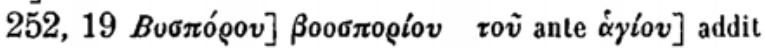

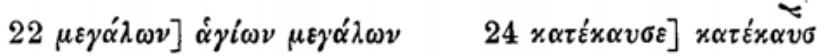

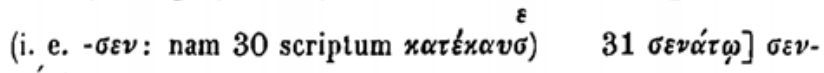
$\nu \alpha \dot{\tau} \tau$.

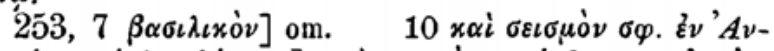

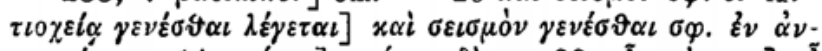

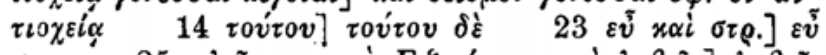

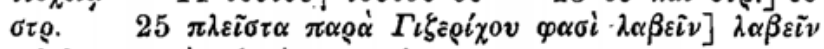
$\pi \dot{\lambda} \varepsilon i \sigma \tau \alpha \pi \alpha \rho \dot{\alpha} \gamma \iota \xi \varepsilon \rho i \chi 0 v$ $\varphi \alpha \sigma i$.

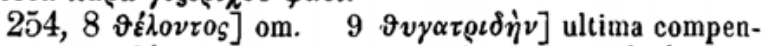
dio, quod $\delta \dot{\eta} \nu$ est potius quam $\delta \dot{\eta} \nu$

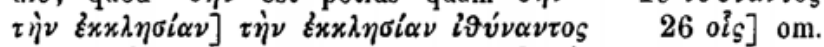
ct $27 x \alpha i \tau \tilde{\eta}_{S}$ pro $\left.\tau \tilde{\eta}_{S} \quad 27 \vartheta \varepsilon \rho \mu \tilde{\eta}_{S}\right] \vartheta \dot{\varepsilon} \varrho \mu \eta$ s recte 28 $\left.\dot{\varepsilon} \pi \iota \beta \lambda \varepsilon^{\prime} \psi \eta\right]$ ह $\left.\dot{\varepsilon} \pi \lambda \alpha^{\prime} \mu \psi \eta \quad 29 x \alpha \tau \alpha \lambda.\right]$ his superscr. col. rubro

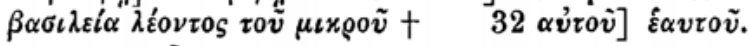

$2555,1 \tilde{\eta} \nu \delta \dot{\varepsilon}]$ super haec novo versu inchoata rubro col.

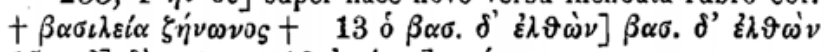

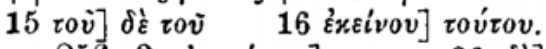

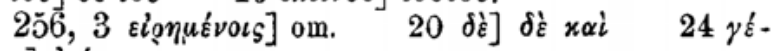

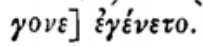



p. $258,8,22$.

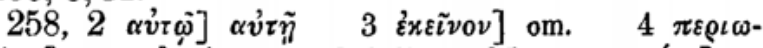
$\delta \iota v i \alpha \iota \varsigma] \pi \varepsilon \rho \omega \delta v v l \alpha \iota \varsigma$, quod dedi $25 x \alpha \tau \varepsilon \sigma \chi \eta x o ́ t \alpha] \tau \varepsilon$ -

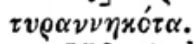

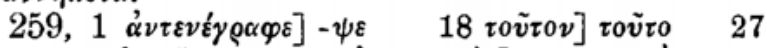

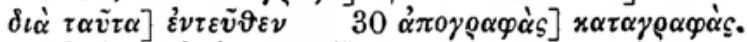

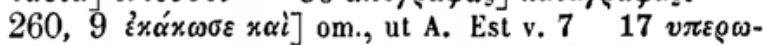

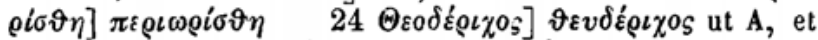

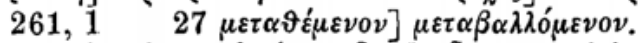

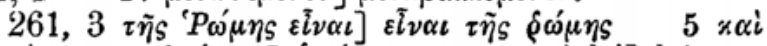

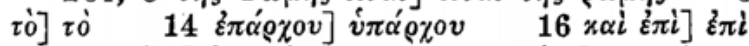

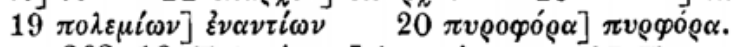

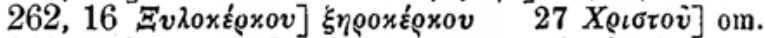

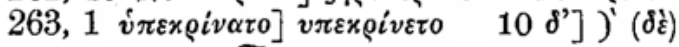

$\left.15 \tau \alpha \gamma \mu \alpha \alpha^{\prime} \omega \nu\right] \tau \alpha \xi \iota \widetilde{\rho} \chi$ ex praeced. versu $21 \delta$ pro $\delta \dot{\varepsilon}$,

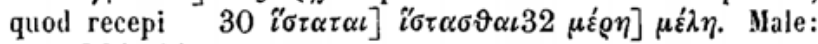
v. p. 264,14 .

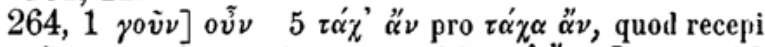
$15 \nu \varepsilon \tilde{\omega} \nu$ pro $\nu \eta \tilde{\omega} \nu$, quod recepi $24 x \alpha \tau^{\prime}$ oै $\left.\nu \alpha \varrho\right]$ om. 32 $\tau \varepsilon \sigma \sigma \alpha \rho \varepsilon \sigma \kappa \alpha i \delta \varepsilon x \alpha] \delta \varepsilon \psi \alpha \tau \varepsilon \dot{\varepsilon} \sigma \sigma \alpha \rho \alpha$.

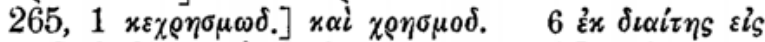

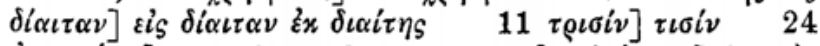

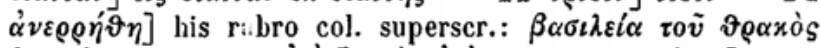

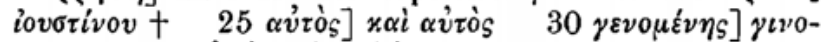

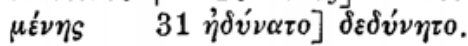

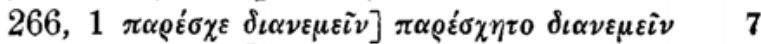

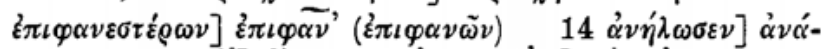
$\left.\left.\lambda \omega \sigma \varepsilon v \quad 17 \delta^{\prime}\right] \delta \dot{\varepsilon} \quad 22 \alpha \dot{\alpha} \nu \gamma . A \dot{v} \gamma.\right] \alpha \dot{v} \gamma . \alpha \dot{\alpha} \nu \gamma \gamma$.

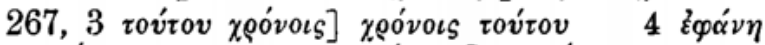

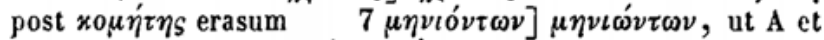
Zonaras alibi $\quad 20 x \alpha \tau \dot{\alpha}]$ x $\alpha \tau \dot{\alpha} \tau \tilde{\omega} \nu \quad 30 \varepsilon i] \mathrm{om}$.

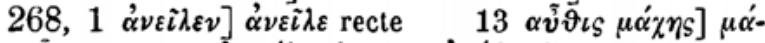

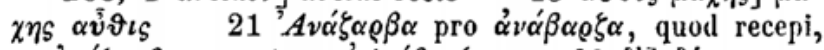

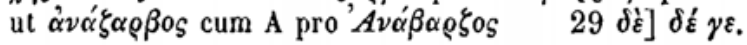

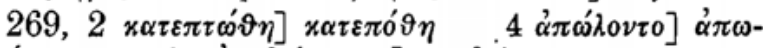

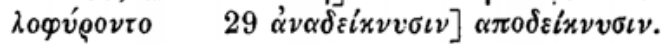




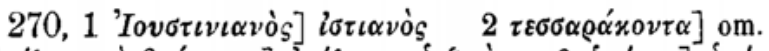

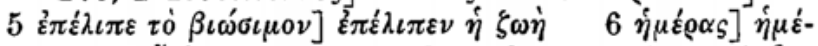

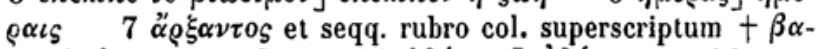

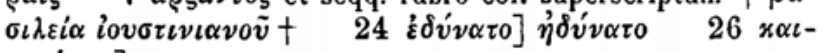
voré $\rho \omega \nu]$ om.

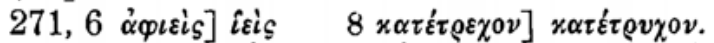

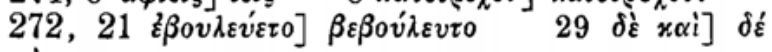
$\gamma \varepsilon x \alpha i$.

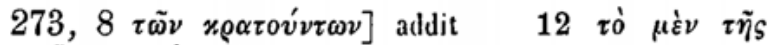
$\tau \tilde{\omega} \nu \sigma \tau.] \quad \tau \tilde{\omega} \nu \mu \dot{\varepsilon} \nu \tau \tilde{\eta} \delta \tau \tilde{\omega} \nu \sigma \tau$., quod recepi, quum eodem ducere videatur A 21 ovoíal] $\pi \varepsilon \rho \iota v v \sigma i \alpha \iota$, quod recipiendum.

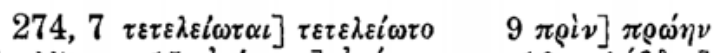

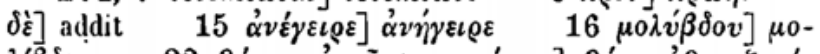

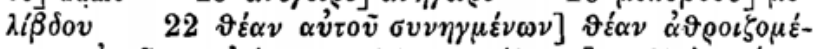

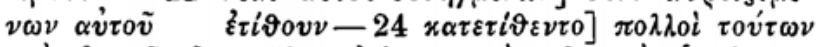

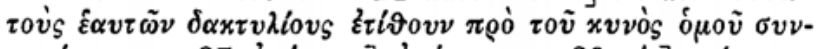

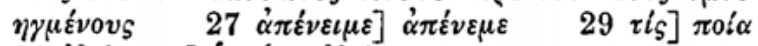

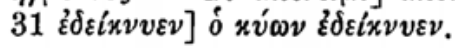

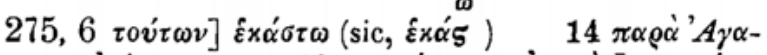

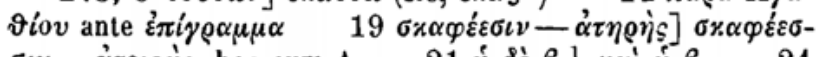

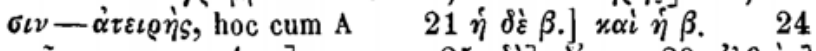

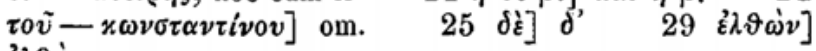
ย่งจ่ั่.

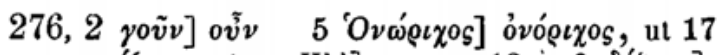

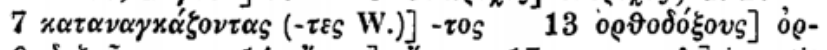

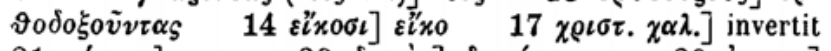

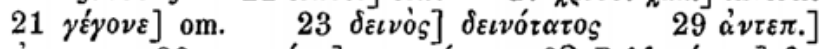

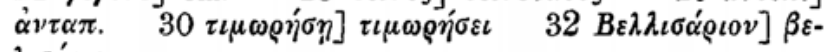

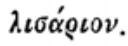

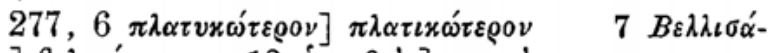

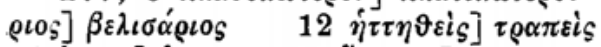

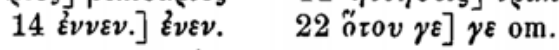

$\left.278,9 \tau \dot{\eta} \nu, \Sigma \alpha \rho \delta \omega]] \sigma \alpha x \varepsilon \rho \delta \omega^{\prime} \quad \alpha \dot{v} r \dot{\eta}\right] \alpha \tilde{v} \tau \eta$ bene

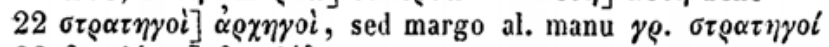
$32 \beta \alpha \sigma \iota \lambda i \sigma \sigma \eta] \beta \alpha \sigma \iota \lambda i \delta \iota$. 


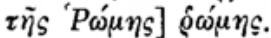

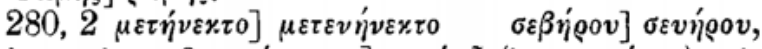

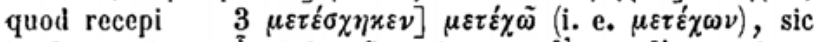
in fine paginae,,$\tilde{\eta} \nu$ infra $\tilde{\omega}$ posito $\delta$ 'pro $\delta \dot{\varepsilon}$, quod re-

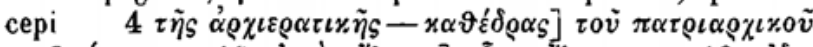

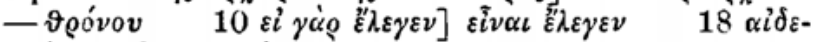

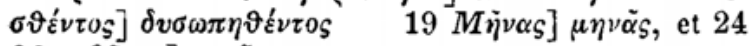

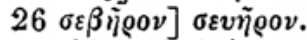

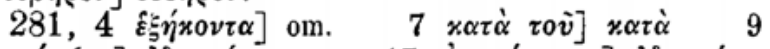

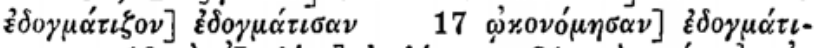

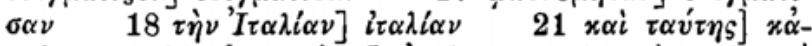

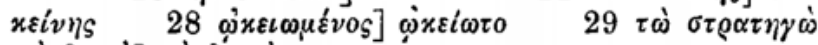
$\tau \dot{\omega} \delta \iota \tau \tau \dot{\omega}] \tau \dot{\omega} \delta_{\imath}^{\prime} \tau \tau \dot{\omega} \sigma \tau \rho$.

282, 4 है

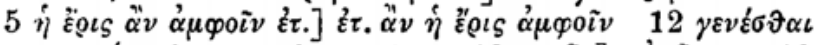
$\begin{array}{lll}\text { pro } \gamma \varepsilon v \eta ́ \sigma \varepsilon \sigma \vartheta \alpha \iota, \text { quod recepi } & 13 \sigma \varphi \tilde{\omega} \nu] \alpha v \tau \tilde{\nu} \nu & 18\end{array}$ $\tau^{2}$

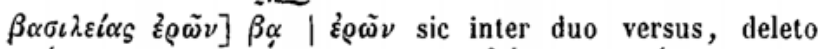
$\tau \varepsilon v ́ \sigma o \nu \tau \alpha$, quod e proximi versus $\varepsilon \omega^{\prime} \alpha \nu \sigma \tau \rho \alpha \tau \varepsilon v ́ \sigma o v \tau \alpha$ initio repetitum. Nihil igitur variat $27 \stackrel{\alpha}{\alpha}\{i \alpha \nu] \pi \alpha \nu \alpha \gamma \tilde{\eta} \quad 28$

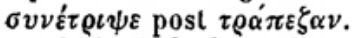

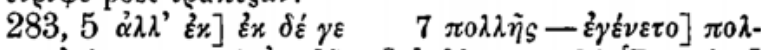

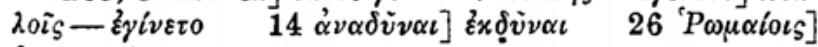

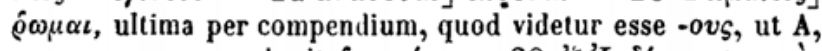
quum versu praeced. sit $\oint \omega \mu \alpha i o u c \quad 28 \xi \xi \xi ' I \nu \delta i \alpha \varsigma$ ante $\pi \rho o_{S}$

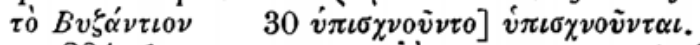

284, 1 ante, non post $\omega^{\alpha} \dot{\alpha}$ interpungit 8 els $\tau \dot{o} B v$ -

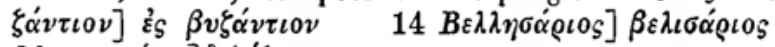
$23 \pi \iota \sigma \tau \varepsilon \dot{v} \varepsilon \iota \nu] \delta 0 \xi \alpha \dot{\alpha} \varepsilon \iota \nu$.

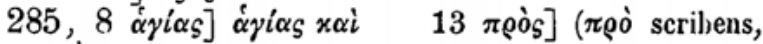

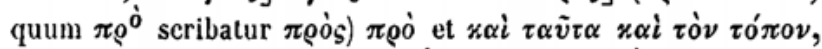

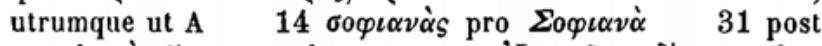

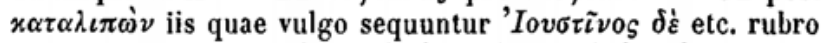

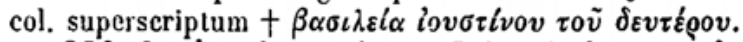

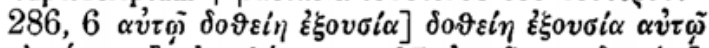
$\left.11 \alpha i \tau \iota \omega \mu \varepsilon \nu 0 \nu]]^{\prime} \alpha i \tau \iota \alpha \vartheta \varepsilon^{\prime} \nu \tau \alpha \quad 27 \varepsilon^{\prime} \tau^{\prime} \tilde{\eta}_{S} \pi \alpha \nu \delta \alpha \iota \sigma i \alpha \varsigma\right] \tau 0 \tilde{v}$

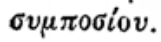


287, $7 \varphi v \lambda \alpha ́ \sigma \sigma \omega \nu \tau \alpha \iota$ pro $\varphi v \lambda \alpha ́ \sigma \sigma o \nu \tau \alpha \iota$, quod recepi

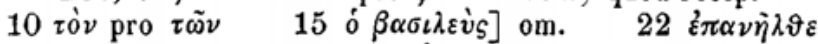

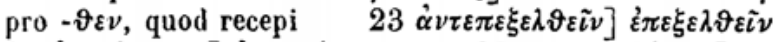

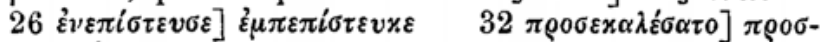

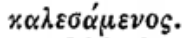

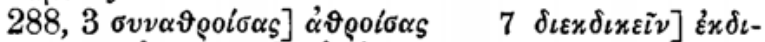

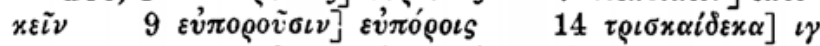
additum $\mathrm{m}$. rec. Sequentibus rubro colore superscriptum

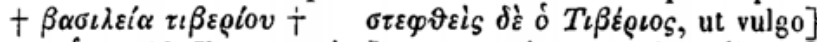
om. $\delta \quad 19 K \omega \nu \sigma \tau \alpha \nu \tau i \alpha \nu] x \omega \nu \sigma \tau \alpha \nu \tau i \nu \alpha \nu \quad 25 \pi \delta$. $1 \varepsilon \mu \nu \nu]$ addit $26 \dot{\eta} \tau \sigma o i \mu \alpha \sigma \varepsilon] \dot{\eta} \tau o i \mu \alpha \zeta \varepsilon \quad \pi \rho \circ \dot{v} \beta \dot{\alpha} \lambda \varepsilon \tau o$ pro $\pi \varrho 0 v-$

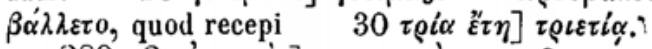

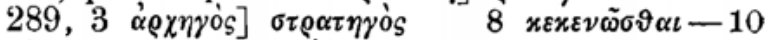

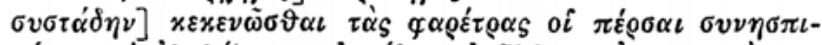

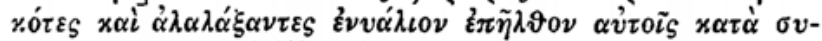

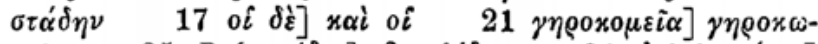

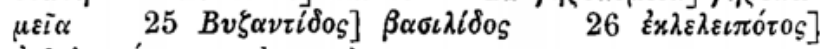

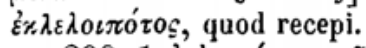

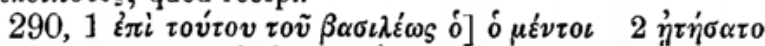

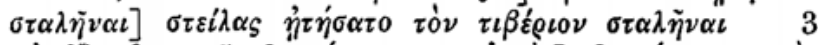

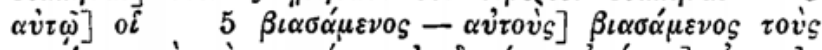

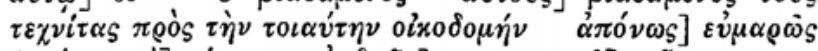

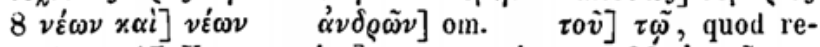

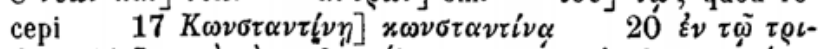

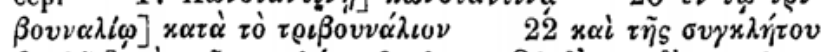

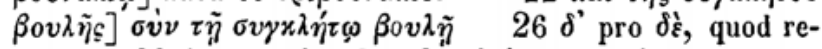
cepi 28 inscr. col. rubro $\beta \alpha \sigma \iota \lambda \varepsilon i \alpha \mu \alpha v \rho \iota x i o v$.

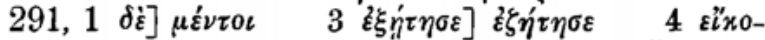

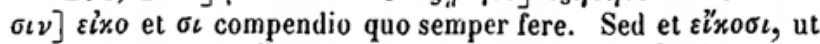
p. 74 A. Scripsi $\varepsilon \iota^{\prime} x 0 \sigma \iota$, et mox $\varepsilon \pi 0 i \eta \sigma \varepsilon \nu$ pro $\dot{\pi} \pi 0 i \eta \sigma \varepsilon$, ubi

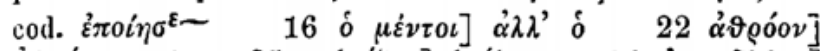

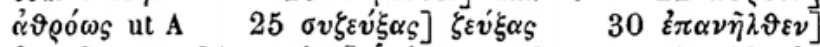

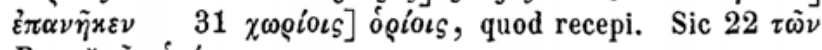

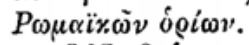

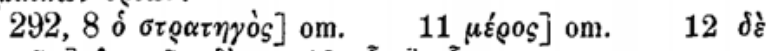

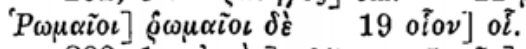

$\left.293,1 \alpha v \tau_{0} v_{s}\right]$ addit $\left.5 \tau \tilde{\omega} \nu\right]$ om. $\alpha \varrho \chi \tilde{\eta} s \hat{\eta} \xi[0 v]$

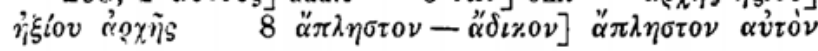




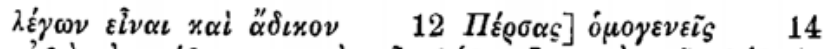

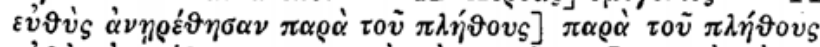

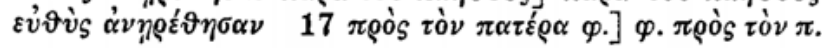

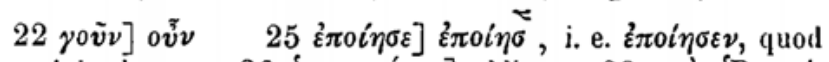

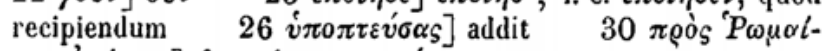

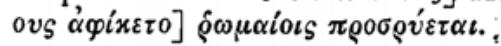

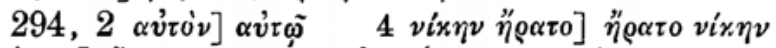

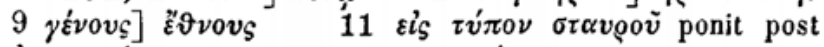
$\dot{\varepsilon} \pi \iota \sigma x v v i \omega \nu$, non, ut ceteri, post $\sigma \tau i \gamma \mu \alpha \sigma \iota v$, quod sequulus

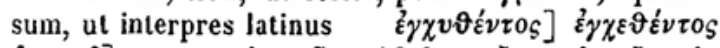

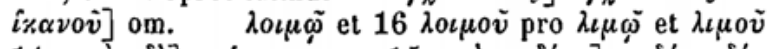

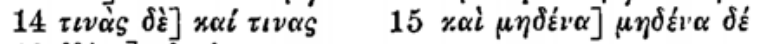
$18 i \delta i \alpha \nu]$ oixci $\alpha \nu$.

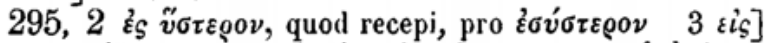
$\left.\dot{\varepsilon}_{S} \quad 4 \tau \dot{\alpha}\right]$ om. $\left.\quad \mu \varepsilon \tau \dot{\alpha} \delta \dot{\varepsilon}-\dot{\varepsilon} \tau \tilde{\omega} \nu\right]$ om. $8 \dot{\eta} \dot{\alpha} \nu \varepsilon \dot{\sigma} \sigma \pi-$

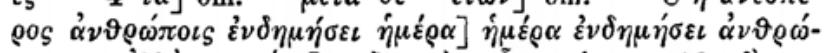

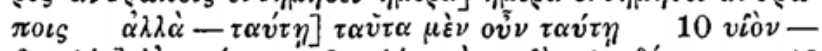

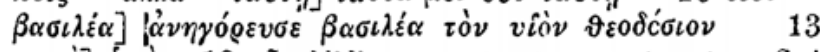
$\pi \alpha \rho \dot{\alpha}] \dot{v} \pi \hat{o}_{0} 18 \tau \tilde{\eta}$ addidi ex eo, ut antea est et postea. Sed rectius fortasse abest, etsi ita $\tau \tilde{\eta}$ ante $B v \zeta \alpha \nu \tau i \delta\llcorner$ ex eodem re-

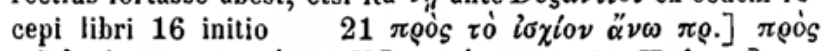

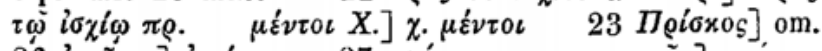

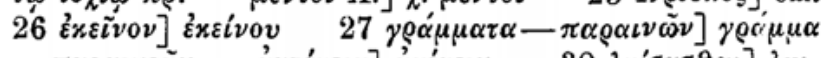

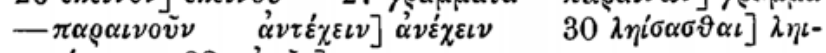

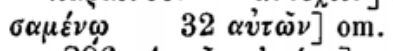

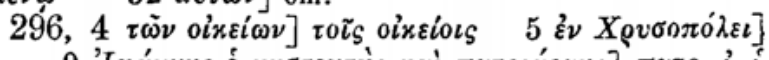

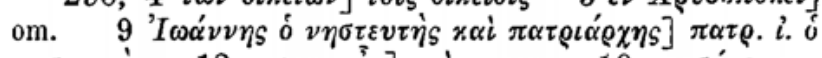
$\nu \eta \sigma \tau \varepsilon v \tau \dot{\eta} \varsigma \quad 12 \pi \rho \circ \varepsilon \chi .0 \bar{v} \nu] x \alpha i \pi \rho \circ \varepsilon . \quad 19 \pi \rho \circ \sigma \nu \varepsilon \gamma * \alpha \nu$

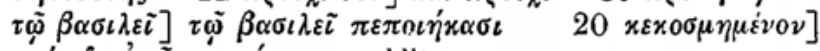

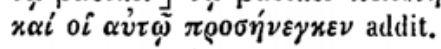

297, 1 ' $\delta^{\prime}$ pro $\delta \dot{\varepsilon}$, quod dedi 8 ' $\left.A \beta \alpha \rho \omega \nu\right] \beta \alpha \rho \beta \alpha \rho \omega \nu$

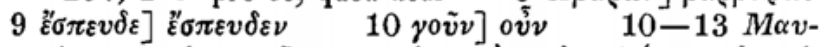

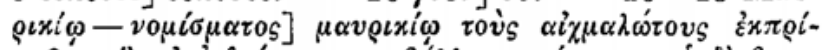

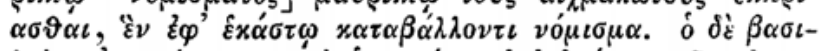

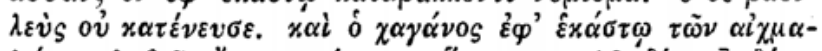

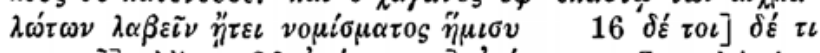
$\tau 0 \tilde{v}]$ addit $\left.26 \alpha^{\prime} \pi \varepsilon^{\prime} \pi \varepsilon \mu \psi \varepsilon \nu\right]{ }^{\prime} \nu \varepsilon^{\prime} \pi \varepsilon \mu \psi \varepsilon$. Ergo delevi $"$. 
298, $2 \pi \rho \circ \varepsilon \lambda \dot{\eta} \lambda v \vartheta \varepsilon v$ 9 qi pro $\varphi$, quod recepi

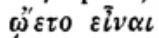
ג̇ev́ov $\sigma \alpha$ $18 \pi \alpha \varrho \varepsilon$

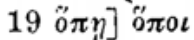

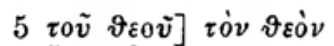

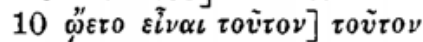

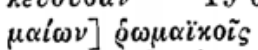

\section{(10xĩ}

10 geto alva touro $x \alpha i] . x \varepsilon-$ $28 \mathrm{P \omega}$ -

24 youv ] ov่v

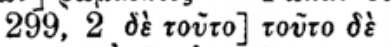
$14 \tau \tilde{\omega} \beta . \alpha \dot{v} \tau$.] $\alpha \dot{v} \tau . \tau \tilde{\omega} \beta$.

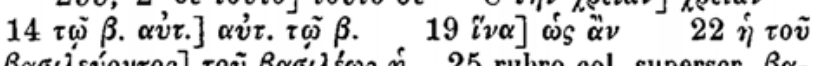
$x \rho \varepsilon i \alpha \nu$

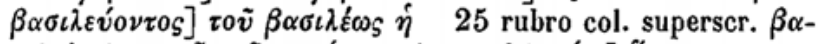

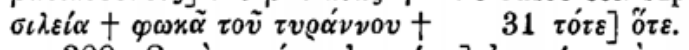

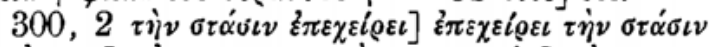

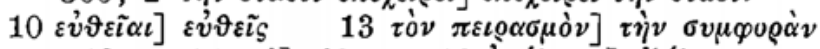
ex v. 12 15 rovi] addit

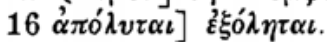

$301,1-3$ post $\sigma \tau \rho \alpha \tau \imath \eta_{s}$ quarta pars versus vacua relicta, ut supra dimidium sequentis, in quo sequitur versus $\alpha \hat{\imath} \alpha \hat{\imath}-$

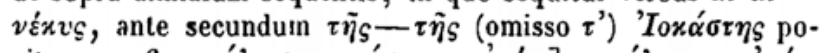

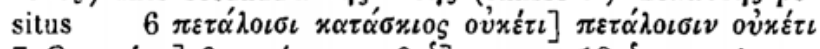

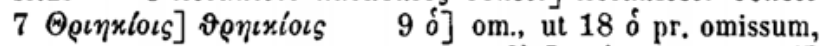

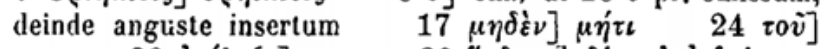

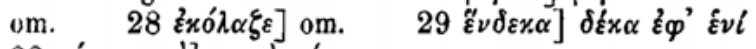

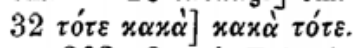

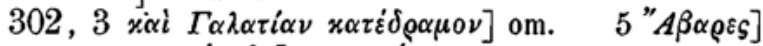

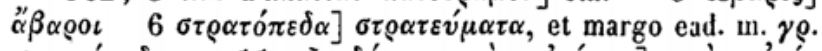

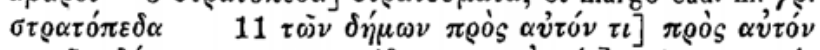

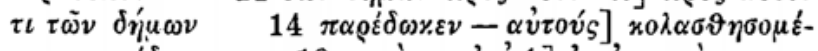

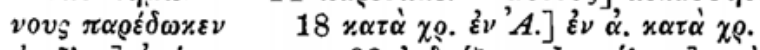

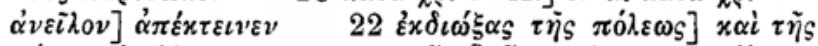

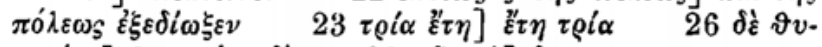
$\left.\left.\gamma \alpha \tau \tau_{\varepsilon}^{\prime} \rho \alpha\right] \vartheta v \gamma \alpha \tau \tau_{\varepsilon} \rho \alpha \delta \dot{\varepsilon} \quad 29 \tau \tilde{\eta}_{S} \Delta.\right] \delta$.

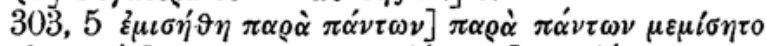

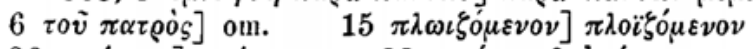

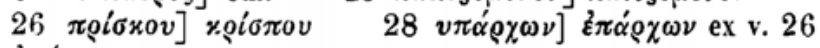

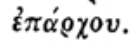

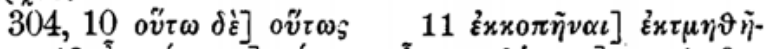

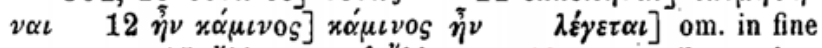
versus $15 \alpha^{\prime \prime} \lambda \lambda_{0 \iota}$ pro of $\alpha^{\prime \prime} \lambda \lambda_{0 \iota} \quad 19$ sequentibus rubro col. superscriptum $+\beta \alpha \sigma \iota \lambda \varepsilon i \alpha \hat{\eta} \varrho \alpha x \lambda \varepsilon i o v$ : $. \quad 23-25 \dot{\eta} \mu \varepsilon^{\prime}-$

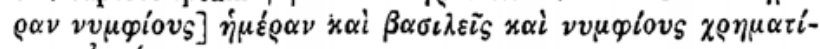

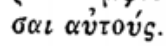




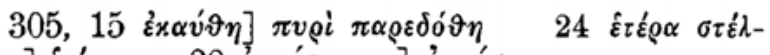

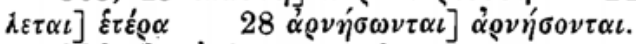

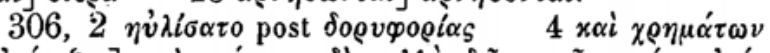

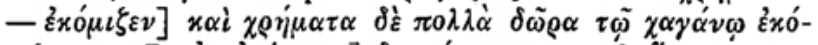

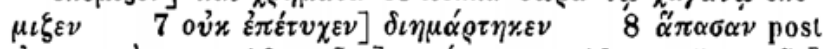

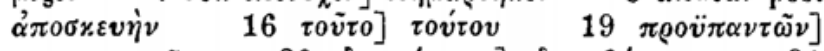

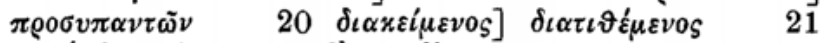

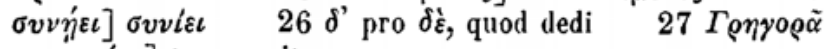
$\pi \alpha \tau \rho\llcorner x i o v]$ transponit.

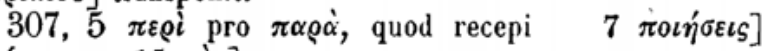

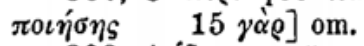

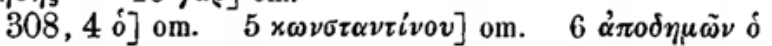

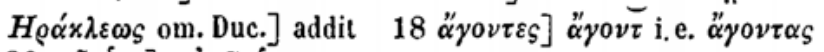
$29 \tau \tilde{\omega} \dot{v} \pi$.] $\alpha \dot{v} \tau \tilde{\omega} \tilde{v} \pi$.

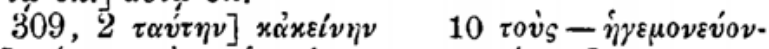
$\left.\left.\tau \alpha_{\xi}\right] \pi \alpha^{\prime} \nu \tau \alpha_{S} \tau o \dot{v}_{S}-\hat{\eta} \gamma \varepsilon \mu o^{\prime} \nu \alpha_{S} \quad 11 \pi \alpha^{\prime} \nu \tau \omega \nu\right]$ om. 13

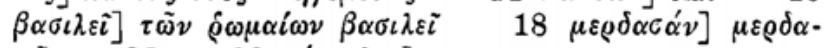

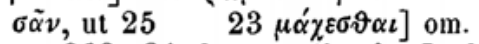

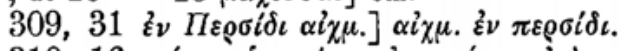

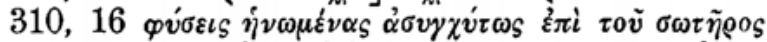

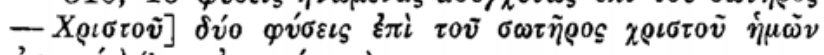
$\dot{\alpha} s v \gamma \chi \dot{v} \tau l$ (i. e. $\left.\dot{\alpha} \sigma v \gamma \chi v v^{\prime} o v s\right)$.

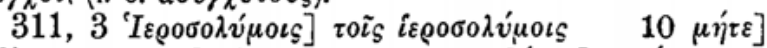

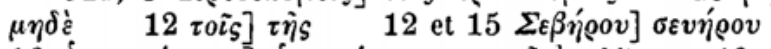

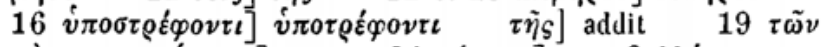
$\left.\gamma \grave{\alpha} \varrho-x \tau \eta \sigma \alpha \dot{\alpha} \mu \nu \sigma_{S}\right]$ om. $\left.24 \pi i \pi \tau \omega \nu\right] x \alpha \tau \alpha \beta \alpha \lambda \lambda o_{\mu} \mu \varepsilon \nu \nu_{S}$ $27 \tau \iota v \iota \delta \dot{\varepsilon}] \tau \iota v t$.

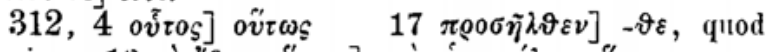

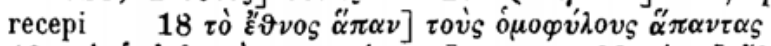

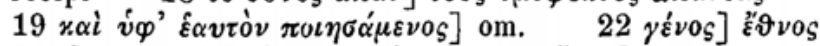

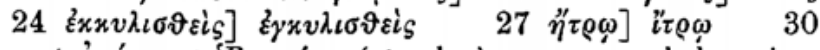
post $\dot{\alpha} \nu v{ }^{\prime} \omega \nu$ et ' $P \omega \mu \alpha i \omega \nu$ (ut vulgo) erasum vocalulum eiusdem fere spatii, quod primo quidem loco manifesto fuit $\dot{\alpha} \nu v ́ \omega v$, fortasse etiam secundo : nam littera prima fuisse videtur $\alpha \quad 32 \mu \varepsilon \tau \alpha \beta \alpha i \nu \varepsilon \iota-313,1 K \omega \nu \sigma \tau \alpha \nu \tau i \nu 0 \nu$ (ut vulgo)] rubro col. superscriptum $\beta \alpha \sigma \iota \lambda \varepsilon \varepsilon^{\prime} \alpha$ x $\omega \nu \sigma \tau \alpha \nu \tau i \nu 0 v$

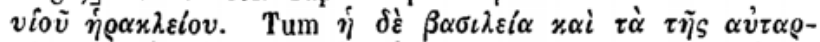

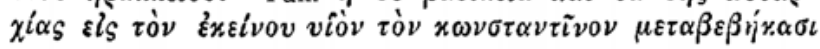




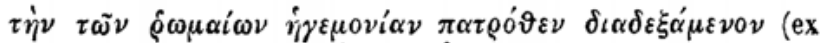

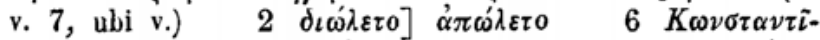
vos] $x \omega \dot{v \sigma \tau \alpha \varsigma} \quad 7 \tau \dot{\eta} \nu-x \alpha x o ́ \delta 0 \xi o v] \tau \tilde{\eta}_{S} \alpha \varrho \chi \tilde{r}_{S}, \alpha \lambda \lambda \lambda^{\prime}$

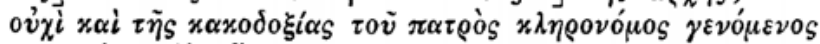

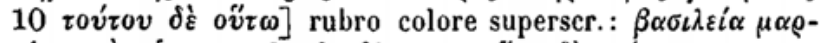

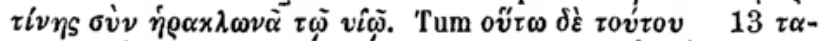

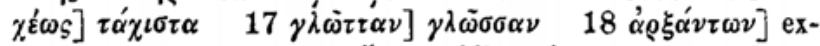
tremo versu post $\alpha \alpha \tau \alpha \psi \eta \varphi i \xi \varepsilon \tau \alpha \iota$ additum in margine, quod om.

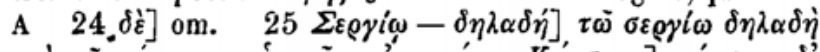

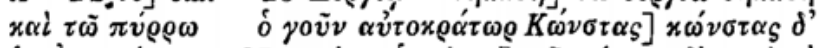

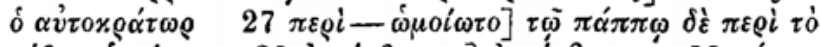

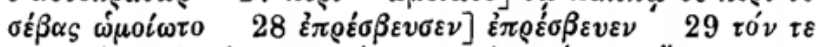

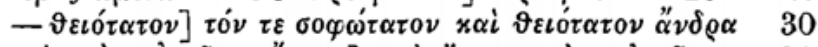

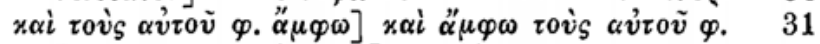

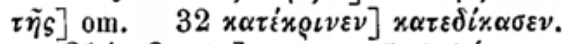

$314,3 \tau \tilde{\omega} \nu]$ om. $\left.5 \vartheta \varepsilon \lambda \eta^{\prime} \sigma \alpha \tau \tau \alpha_{S}\right] \vartheta \varepsilon \lambda \eta^{\prime} \sigma \alpha \nu \tau$ $\left.M \alpha \beta i \alpha_{S}\right] \mu \alpha v i \alpha_{S}$ constanter $\pi 0 \lambda \varepsilon \mu i \sigma \tau \eta \rho i \alpha_{S} \quad \pi \lambda \varepsilon i \sigma \tau \alpha S$

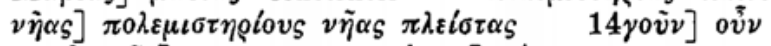

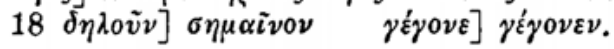

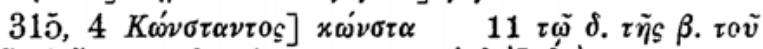

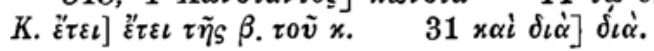

316, 16 sequentibus rubro col. superscr.; $\beta \alpha \sigma \iota \lambda \varepsilon i \alpha$ $\lambda$

(compendio $\beta \alpha \sigma i ̈)$ $\alpha \nu \sigma \tau \alpha \nu \tau i \nu 0 v$ (compendio expressis litt. $\tau i$ -

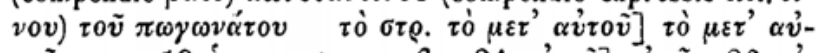

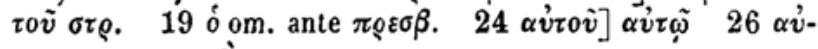
$\tau \dot{v} \nu \delta \dot{\varepsilon}] \alpha v_{\tau} \boldsymbol{\varepsilon}^{\dot{\tau}}$, accentu in ov ex acuto in gravem mutato $27 \dot{v} \pi \varepsilon \rho \beta \varepsilon \beta \eta x \tau^{\prime} \alpha \dot{\alpha} \nu$ in margine duorum versuum exitu et

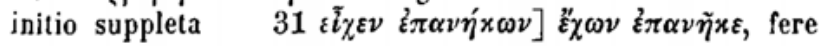
ut A.

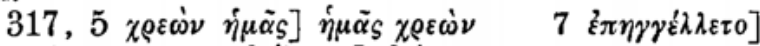

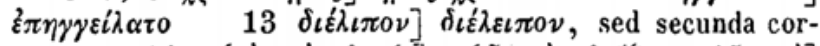

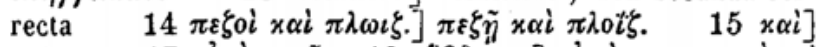

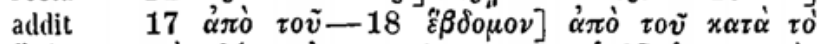

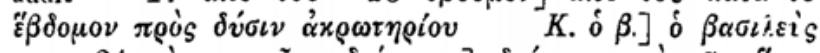

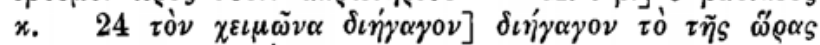

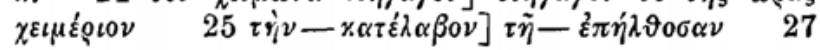




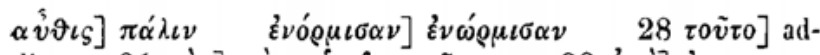

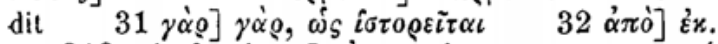

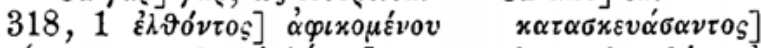

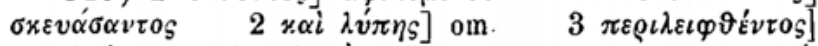
$\pi \varepsilon \rho \iota \lambda \circ i \pi 0 v \quad 4 \sigma x \lambda \eta \rho \dot{\alpha}$ post $\pi \varrho 0 \sigma \pi \varepsilon \sigma o \tilde{v} \sigma \alpha \quad 6 \pi \rho \circ \sigma \alpha \rho \alpha^{\prime}-$

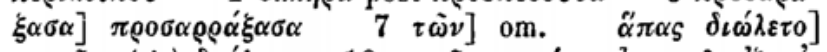

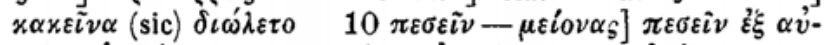

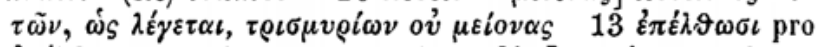

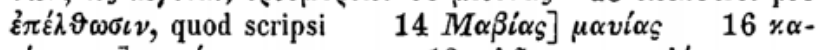

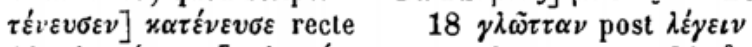

$19 \pi \lambda \eta \varrho \omega \sigma \sigma \alpha \nu \tau \alpha] \pi \lambda \eta \varrho \omega ́ \sigma 0 \nu \tau \alpha$, quod recepi $20 \delta \varepsilon \chi \vartheta \varepsilon i$ S

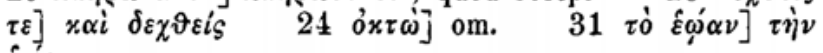
$\varepsilon \omega \dot{\alpha} \alpha \nu$.

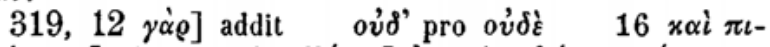

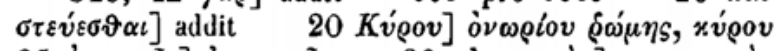

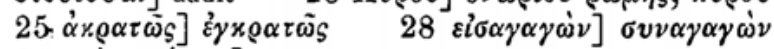

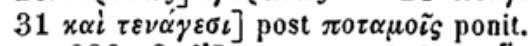

$\left.320,8 \delta^{\prime}\right]$ om. $\quad$ om

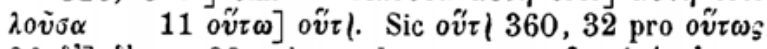

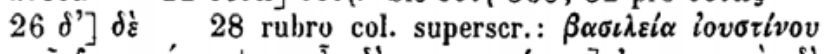

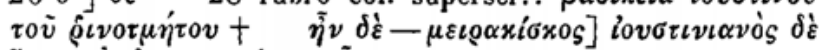

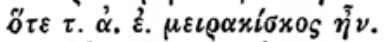

$\left.321,3 \tau \varepsilon \tau v \chi \eta^{\prime} x \alpha \sigma \iota\right] \tau \varepsilon \tau v \chi \dot{\eta} x \alpha \sigma \iota \nu$ et mox $\alpha$ $\rho \tau \iota ~ \delta \dot{\varepsilon} \tau \tilde{\omega} \nu \sigma$.

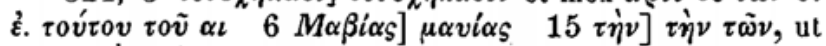
A pro $\tau \dot{\eta} \nu \tau \tilde{\omega} \nu$, quorum alterum delevi, ut est p. 94, A 16

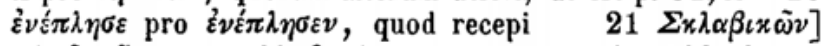
$\vartheta \lambda \alpha \beta \iota x \tilde{\omega} \nu$, ноn $\sigma \vartheta \lambda \alpha \beta \iota x \omega \nu$, ut A et ipse infra 322,21

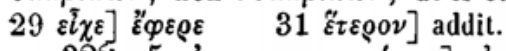

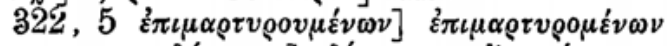

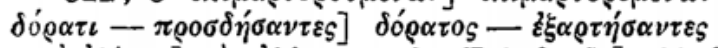

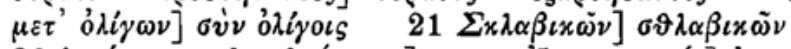

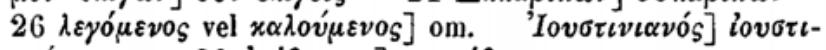

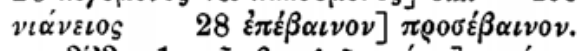

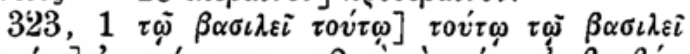

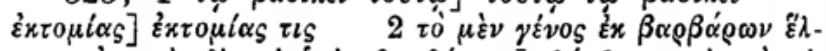

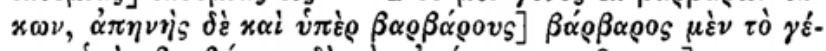

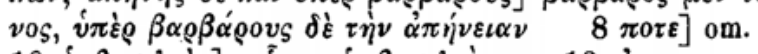

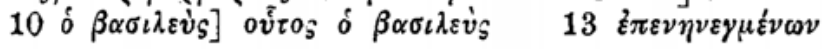




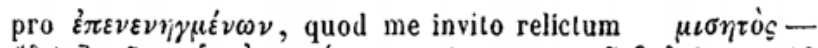

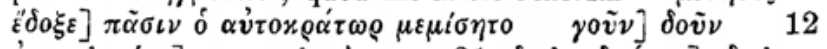

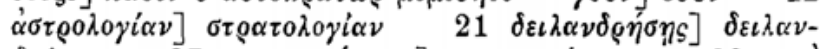

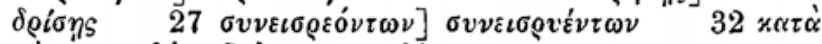

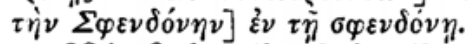

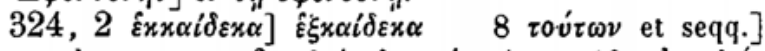
rubro col. superscr.: $\beta \alpha \sigma i \lambda \varepsilon i \alpha$ $\lambda \varepsilon o v \tau i o v+10 \alpha i x \mu \alpha \lambda \omega$ -

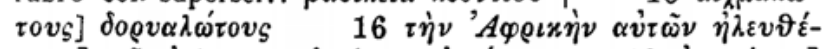

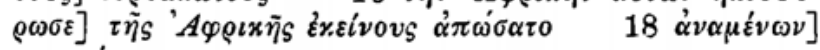
$\pi \rho \circ \sigma \mu \varepsilon \dot{\varepsilon} \nu \nu$.

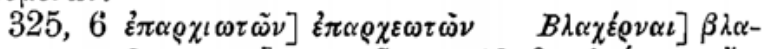

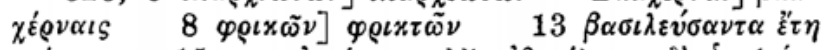

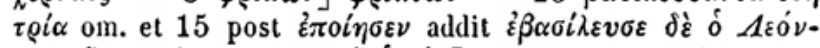

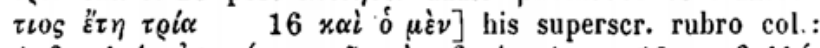

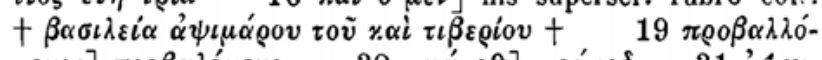

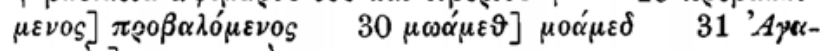

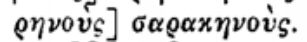

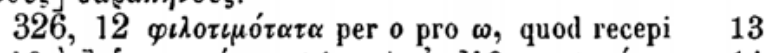
$\left.\alpha \pi \varepsilon \lambda \vartheta \omega^{\prime} \nu\right] \dot{v} \pi 0 \chi \omega \rho \dot{\gamma}^{\prime} \sigma \alpha \varsigma$ et inserit $\alpha \pi \dot{\eta} \lambda \vartheta \varepsilon$ post $\chi \omega^{\prime} \rho \alpha \nu$ et 14 $x \alpha i \mu \varepsilon \tau \dot{\alpha}$ pro $\mu \varepsilon \tau \alpha^{\prime} \quad 19$ ö pro ô $\nu$, quod recepi.

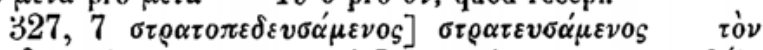

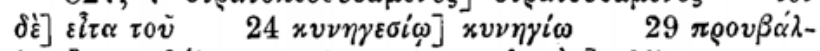

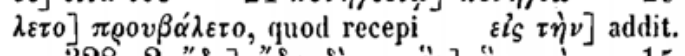

$328,2 \ddot{\eta} \delta \eta] \ddot{\eta} \delta \eta \delta \dot{\varepsilon}$ ö $\nu]$ ôv $x \alpha i \quad 15 \sigma v v \varepsilon x \lambda \varepsilon i-$ $\sigma \vartheta \eta \sigma \alpha \nu] \sigma v \nu \varepsilon x \lambda \varepsilon i \sigma \vartheta \eta$ in fine versus $26 \pi \alpha \rho \alpha \lambda \alpha \beta o ́ v \tau \varepsilon s]$ $\left.\pi \alpha \varrho \varepsilon \iota \lambda \eta \varphi \sigma^{\prime} \varepsilon \varsigma \quad 28 \mu \varepsilon \iota \rho \alpha x i \omega \nu\right] \mu \varepsilon \iota \rho \alpha x i \sigma x \omega \nu$.

$329,5$ है $\iota$ ] om. $\quad 8 \pi \varepsilon \rho \iota 0 \iota \sigma \vartheta \tilde{\eta} \nu \alpha \iota] \pi \varepsilon \rho \iota \rho \iota \sigma \vartheta \tilde{\eta} v \alpha \iota$

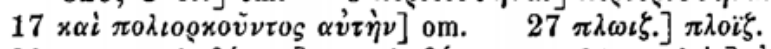

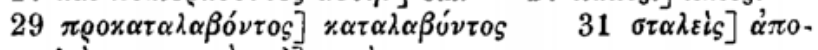
$\sigma \tau \alpha \lambda \varepsilon \dot{i} S \mu \varepsilon \tau \dot{\alpha} \tau o \dot{v}] \mu \bar{c} \tau \dot{\alpha}$.

330,13 sequentibus rubro col. superscr. $\beta \alpha \sigma \iota \lambda \varepsilon i \alpha \alpha \iota-$

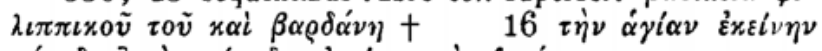

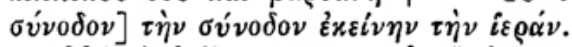

$331,1 \varepsilon \pi i]$ oin. $2 \alpha v i \hat{\omega}] \hat{\varepsilon} \alpha v \tau \tilde{\omega}$, recte fortasse $6 \alpha \dot{\alpha} \pi \varepsilon v o ́ \sigma \tau \eta \sigma \alpha \nu]$,

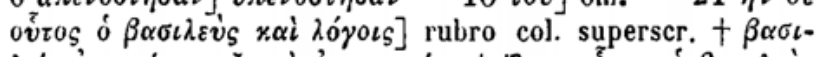

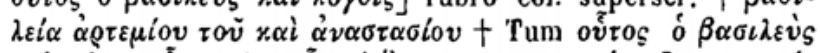

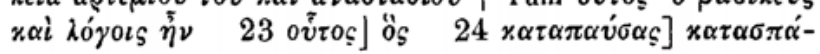


$\sigma \alpha \varsigma \quad 28 \dot{\alpha} q \alpha \iota \rho \varepsilon \vartheta \tilde{\eta} \nu \alpha \iota] \dot{\alpha} \varphi \alpha \iota \rho \varepsilon \vartheta \varepsilon^{\prime} \nu \tau \alpha \quad 30 \pi \rho \omega \sigma \omega \omega^{\prime} \eta_{i-}$ $\sigma \alpha \nu] \pi \rho \circ \sigma \omega \rho \mu \iota \sigma \alpha \nu$, quod recepi. Est paullo ante, p. 325,4

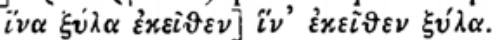

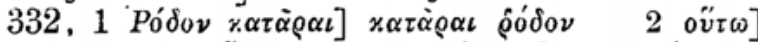

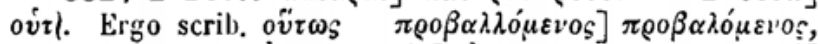

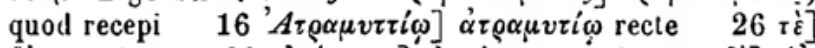

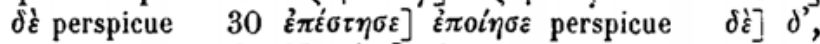

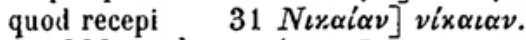

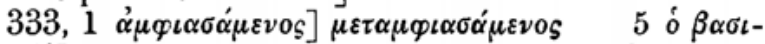

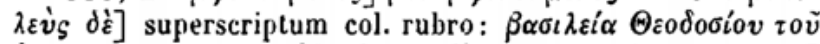

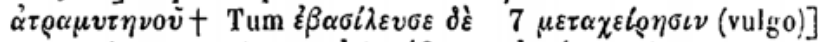

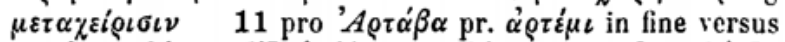

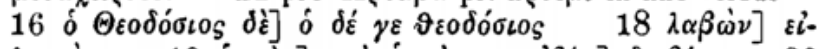

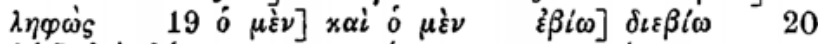

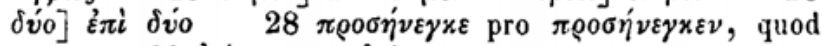

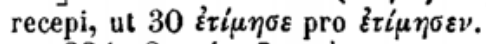

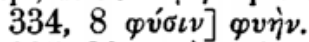

$335,29 x \alpha \tau \dot{\alpha} \tau 0 \dot{v}] x \alpha \tau \dot{\alpha}$.

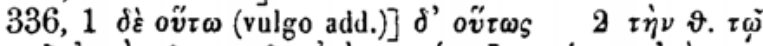

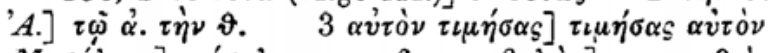

$\left.\left.M \alpha \sigma \alpha^{\prime} \lambda \mu \alpha \varsigma\right] \mu \alpha^{\prime} \sigma \alpha \lambda \mu \alpha \varsigma \quad 6 \pi \rho 0 \sigma \beta \alpha \lambda \dot{\omega} \nu\right] \pi \varepsilon \rho \alpha \iota \omega \vartheta \varepsilon i \varsigma$, ex

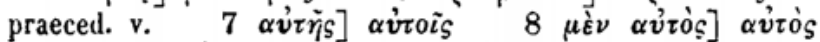

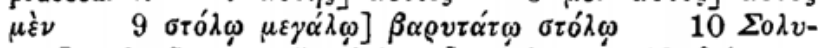
$\left.\mu \tilde{\alpha} \varsigma] \sigma 0 \lambda \iota \mu \tilde{\alpha}_{S} \quad 15 \pi \lambda \varepsilon \dot{i} \sigma \tau 0 \nu\right] \pi \dot{\lambda} \varepsilon i 0 \nu \quad 18 \delta \varepsilon i \sigma \alpha \nu \tau \varepsilon S$

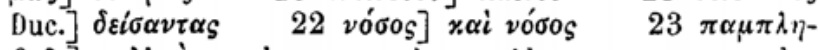
$\vartheta \varepsilon i \varsigma] \pi 0 \lambda \lambda_{0} \dot{v}_{\varsigma}$, sed margo ead., ut videtur, m. $\gamma \rho . \pi \alpha \mu \pi \lambda \eta$

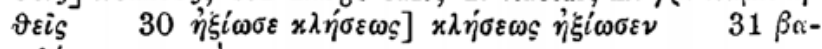
$\sigma \iota \lambda \hat{k} \alpha$ ante $\pi \alpha \rho \dot{\alpha}$.

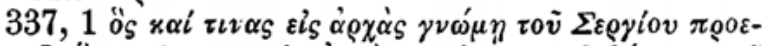

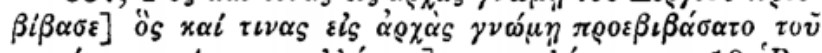

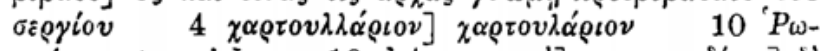
$\mu \alpha i o \iota s$ ante $\left.x \alpha \lambda \dot{\omega} S \quad 19 \varepsilon^{2} \tau i \mu \eta \eta \alpha \nu \alpha \alpha i\right]$ om. $\left.\quad \delta \dot{\varepsilon} \gamma \dot{\varepsilon}\right] \delta \dot{\varepsilon}$

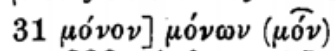

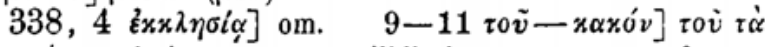
$\ddot{\alpha} \gamma\llcorner 0 \nu$ (recentissima manu et pallidissimo atramento $\alpha$ factum

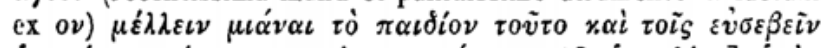

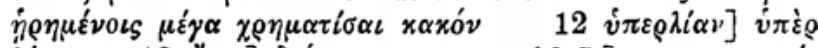

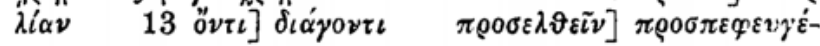




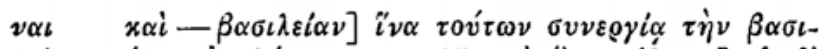

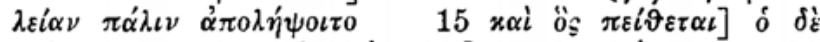
$\left.\pi \varepsilon i \vartheta \varepsilon \tau \alpha \iota \quad 16 \pi \rho \circ \sigma \delta \varepsilon \vartheta \vartheta \eta^{\prime} \sigma \varepsilon \sigma \vartheta \alpha \iota\right] \pi \rho 0 \sigma \lambda \eta \varphi \vartheta \eta^{\prime} \sigma \varepsilon \sigma \vartheta \alpha \iota \quad 22$

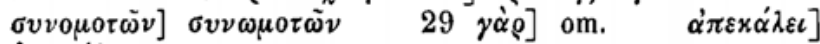
ò $\nu \mu \alpha \dot{\alpha} \xi \omega \nu$.

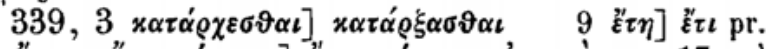

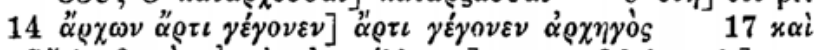

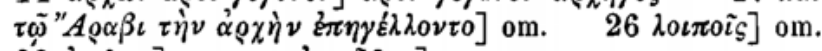

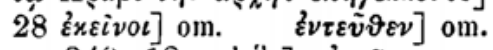

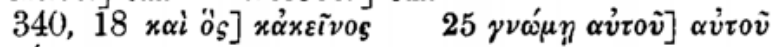

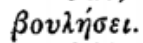

$\left.341,5 \pi \alpha \iota \delta \varepsilon i \alpha_{S}\right] \sigma o \cos ^{\prime} \alpha_{S}$ ex p. $340,29 \quad 8 \mu \varepsilon \tau \alpha-$

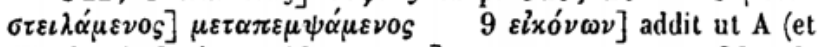
Wolfius in lat.) $18 \pi \varrho \circ \sigma \circ \mu$.] $\pi \varrho \circ \sigma \omega \mu$. recte $23 \tau 0 i_{S}$ (ut W. et Duc. tacente Haasio)] $\tau \alpha i$ s recte.

$342,10 x \alpha i]$ addit $13 \pi \alpha \rho . \tau \tilde{\omega} \nu \Gamma$. $\alpha \dot{v} \tau 0 \tilde{\iota} \varsigma] \tau \tilde{\omega} \nu$

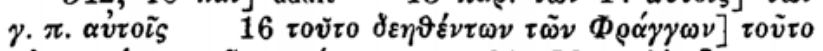

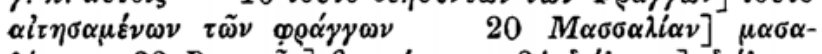

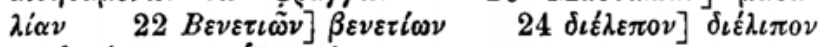

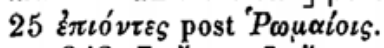

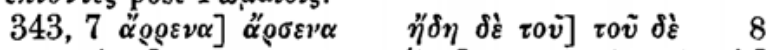

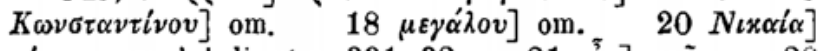
vix $\alpha \iota \alpha$, quod dedi, ut p. 331, $32 \quad 21$ ov $v]$ youv 28 $\beta \alpha \sigma \iota \lambda \varepsilon i]] \beta \alpha \sigma \iota \lambda_{\iota x \tilde{\omega}} \tau \alpha \mu \varepsilon i \omega$.

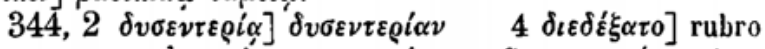

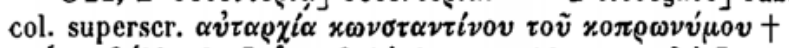
6 i $\left.\pi \varepsilon \rho \beta \alpha^{\prime} \lambda \lambda \varepsilon \sigma \vartheta \alpha \iota\right]$ v $\left.\pi \varepsilon \rho \beta \alpha \lambda \varepsilon \sigma \vartheta \alpha \iota \quad 10 \mu 0 \nu 0 \varepsilon \iota \delta \varepsilon i \zeta\right] \mu 0 \nu 0-$

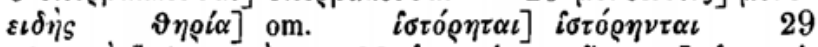

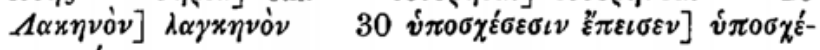
$\sigma \varepsilon \sigma \iota \pi \varepsilon^{\prime} \pi \varepsilon \iota \kappa \varepsilon \nu$.

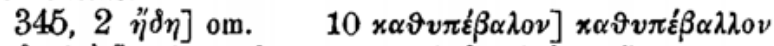

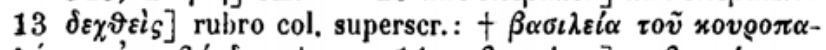

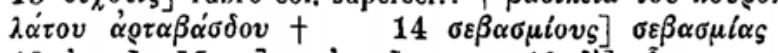

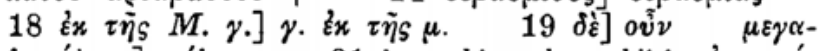
$\left.\lambda_{0} \sigma^{\prime} \lambda_{\varepsilon \omega s}\right] \pi o^{\prime} \lambda \varepsilon \omega s \quad 31$ in verbis vulgo additis $\alpha \nu \iota \chi v \iota \alpha-$ $\sigma \tau \omega \nu] \dot{\alpha} \nu \varepsilon \xi \iota \nu \nu \alpha^{\prime} \sigma \tau \omega \nu$.

346,1 toij] om. ut $\mathrm{A}$, in fine versus et margine ponens

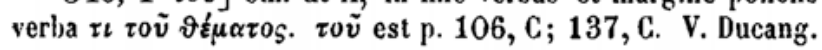


in 'O $\psi: \iota x$, qui utrumque ponit, sed de suo $8 \dot{\alpha} \nu \alpha \sigma x \alpha \psi \alpha \iota]$

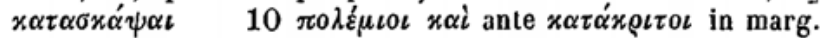
suppleta $\mathrm{m}$. recentissima atramento pallidissimo 16

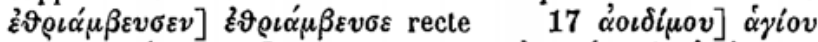

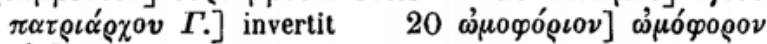
$29 \tau \dot{\eta} \nu] \mathrm{om}$.
347,2 華] $\eta^{n}$

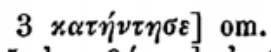
4 o[óv] o[ov sic cum sp. leni

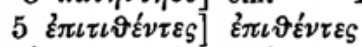

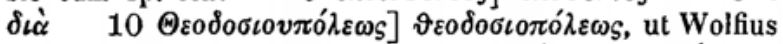

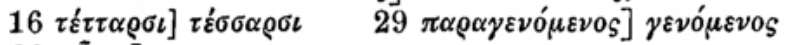
30 oṽนos] om.

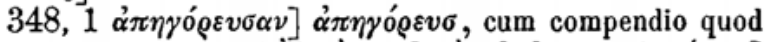

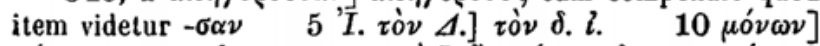

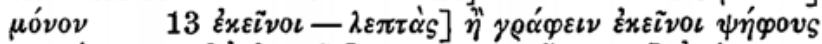

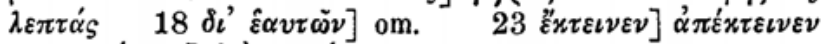
$27 \vartheta \alpha \lambda \dot{\alpha} \sigma \sigma \eta \zeta] \delta \iota \dot{\alpha} \vartheta \alpha \lambda \dot{\alpha} \sigma \sigma \eta s$.

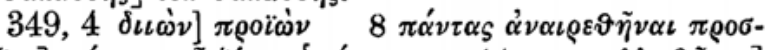

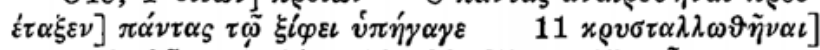

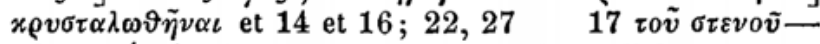
$18 \chi \varrho v \sigma o ́ \pi 0 \lambda_{\iota \nu}$ in marg. manu recentissima pallido atramento

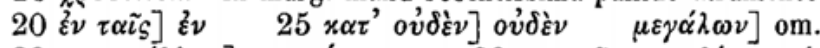

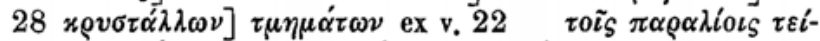

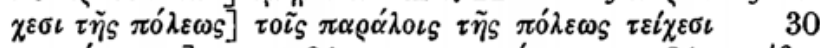

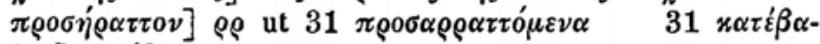
$\lambda o v] x \alpha \tau \varepsilon \beta \alpha \lambda \lambda o v$.

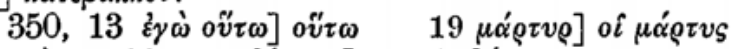

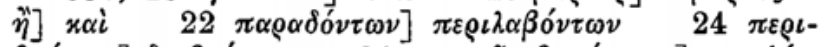

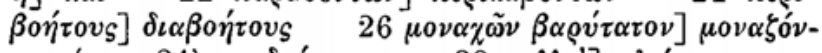

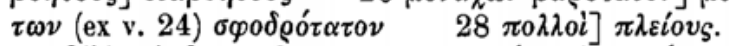

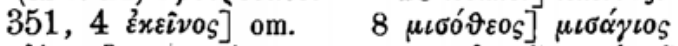

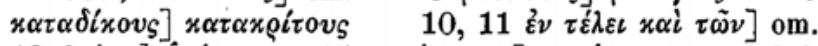

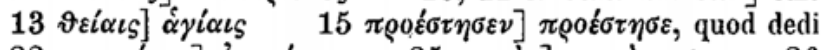

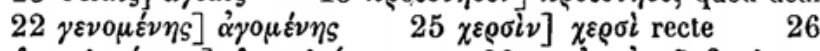

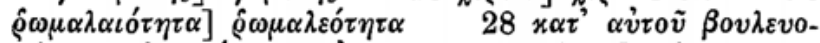

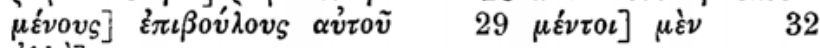
$\dot{\alpha} \lambda \lambda \dot{\alpha}]$ om.

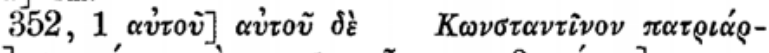

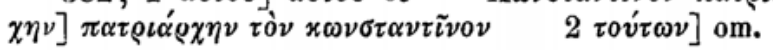




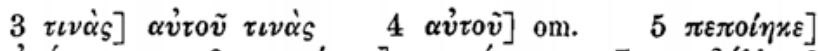

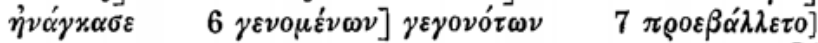

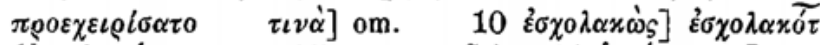

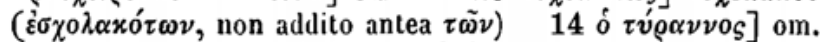

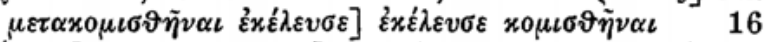
$\left.\pi \lambda \eta^{\prime} \vartheta 0 v \varsigma\right]$ om. $\left.\left.\pi 0 \lambda \lambda_{0} \tilde{v}\right] \pi 0 \lambda \lambda \tilde{\omega} \nu \quad \sigma v v \alpha \vartheta \rho o \iota \sigma \vartheta \varepsilon v \tau 0 \varsigma\right]$

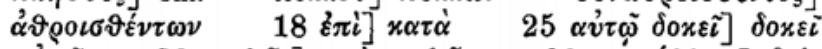

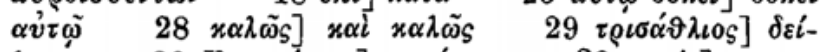

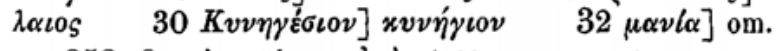

$353,8 x \alpha i$ x $\alpha \dot{\varepsilon} \sigma \alpha \varsigma \alpha \dot{v} \tau \dot{\eta} \nu$ (addito hoc quod deest ceteris)

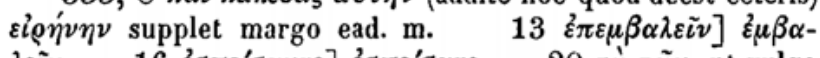

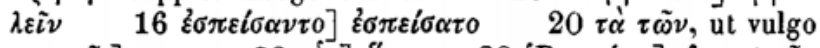
pro $\tau \tilde{\omega}$,$] om. 22$ '

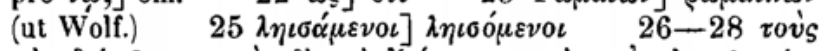

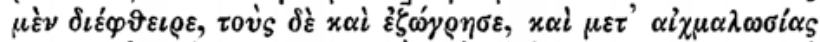

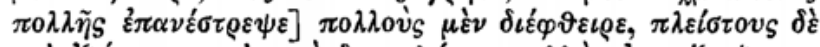

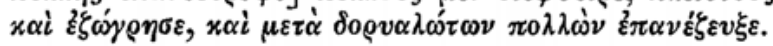

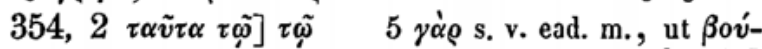
$\lambda \varepsilon \sigma \vartheta \alpha \iota \pi \rho \circ \sigma \varepsilon \lambda \vartheta \varepsilon \tilde{\imath} \nu$ in fine versus addit margo $\left.6 \varepsilon^{2} x \tau \tilde{\omega} \nu\right]$ $\left.\tau \tilde{\nu} \nu 7 \vartheta \alpha \varrho \eta^{\prime} \sigma \varepsilon \iota \varepsilon \nu\right] \vartheta \alpha \rho \varrho \sigma^{\prime} \sigma \varepsilon \varepsilon \varepsilon$, quod dedi $\left.16 \lambda \alpha^{\alpha} \beta \rho \circ \iota\right]$

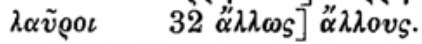

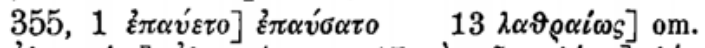

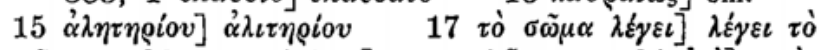

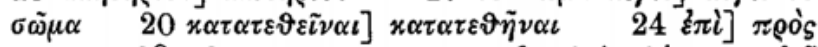

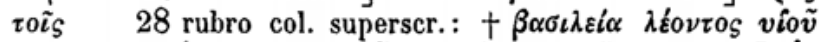

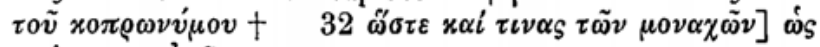
$x \alpha i \tau \iota v \alpha S \alpha v \tau \tilde{v} v$.

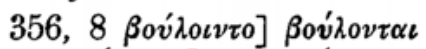
$\left.13 \pi \varrho \circ \varepsilon \sigma \tau \eta^{\prime} x \varepsilon \sigma \alpha \nu\right] \pi \varrho 0 \varepsilon เ \sigma \tau \eta^{\prime} x \varepsilon \iota \sigma \alpha \nu$ $\beta \alpha \sigma \iota \lambda \varepsilon \dot{v} s] \lambda \varepsilon^{\prime} \omega \nu$ $15 \tau \eta v]$ om.

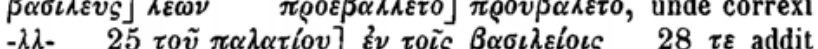

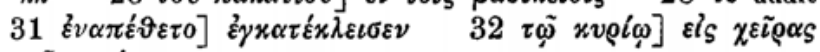

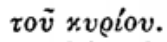

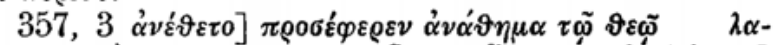

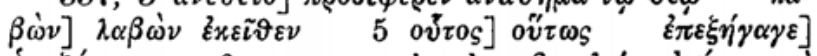

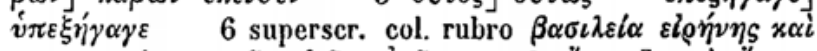

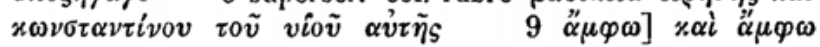




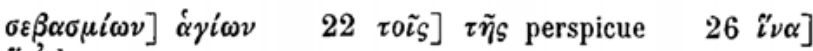
¿" $\nu$ ' bene.

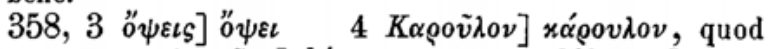
dedi $\left.7 \pi \alpha \tau \alpha \lambda \iota \pi \sigma \tilde{\sigma} \sigma \alpha] \varepsilon^{z} \alpha \sigma \alpha \sigma \alpha \quad 8 \pi \varepsilon \rho \iota \beta \varepsilon \beta \lambda \eta \tau \alpha \iota\right] \pi \varepsilon \rho l-$

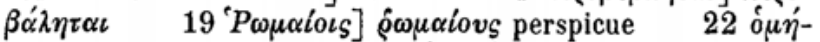

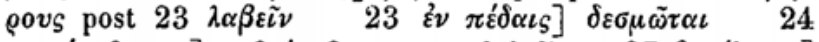

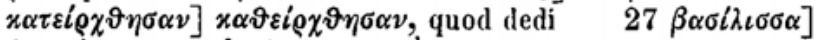

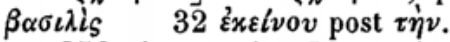

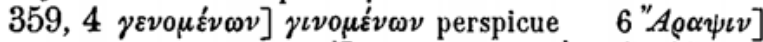

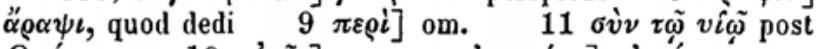

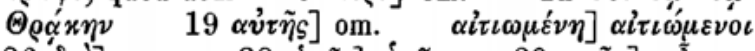

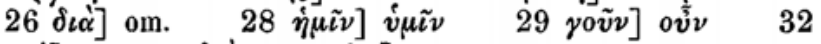

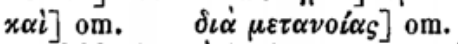

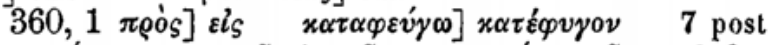

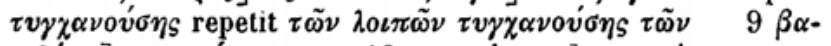
$\sigma \iota \lambda \dot{\varepsilon} \omega \nu]$ ]

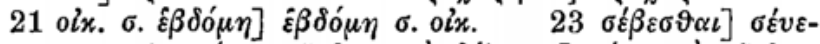

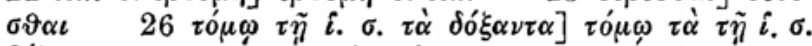
$\delta o ́ \xi \alpha v \tau \alpha$, fere ut $\dot{A} \quad 31 x \alpha i$ addit ut $\mathbf{A}$, servans ante id omissum ab illo $\alpha \dot{v} \tau i$ s $\alpha$.

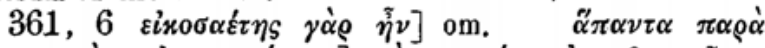

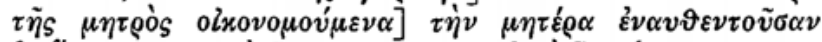

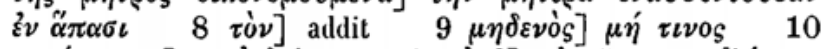

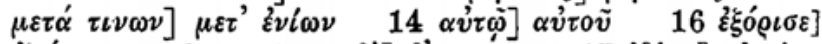

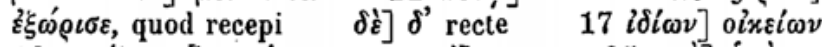

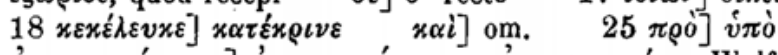

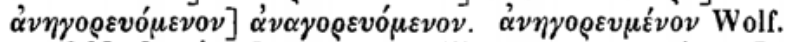

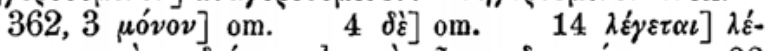
 $\tau \dot{\alpha}$ addit post $\ddot{\eta} \delta \eta \quad 29 \tau \dot{0} \nu] \tau \tilde{\omega} \nu$.

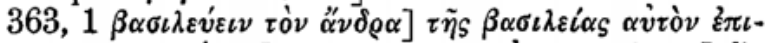

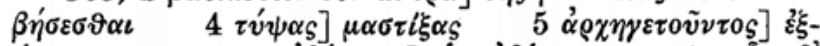

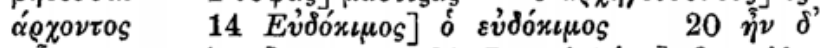

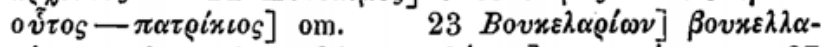

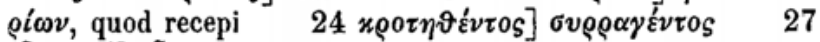

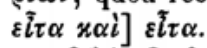

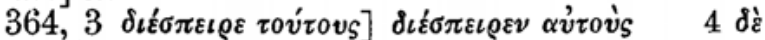

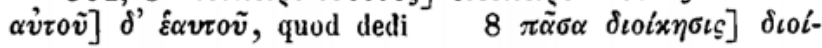




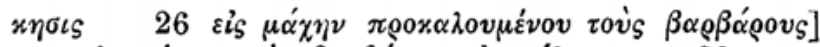

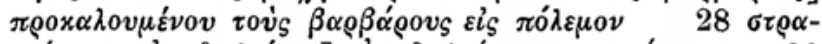

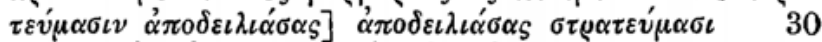

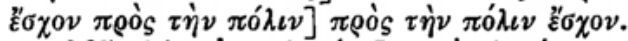

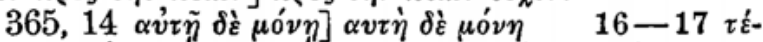

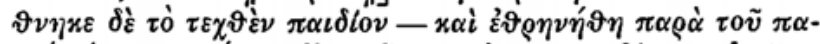

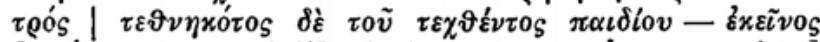

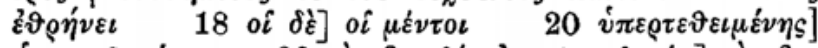

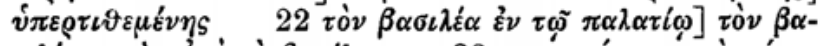

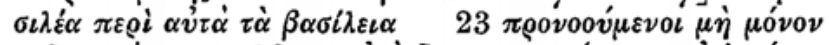

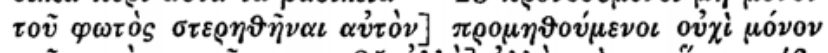
rov

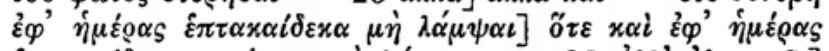

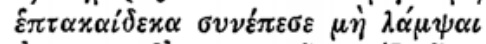

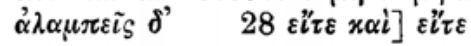
$\left.26 \alpha \lambda \lambda \lambda^{\prime} \alpha \lambda \alpha \mu \pi \varepsilon \tilde{i}_{S}\right]$

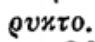

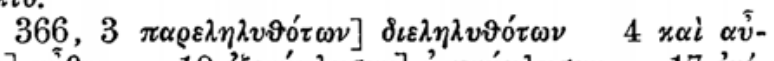

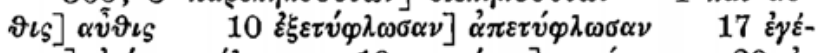

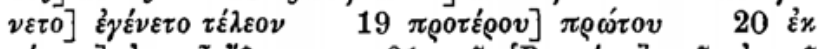

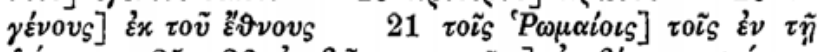

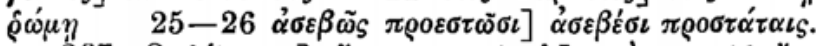

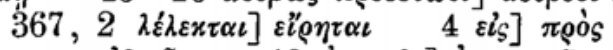

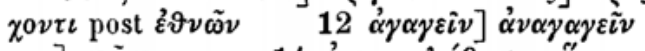

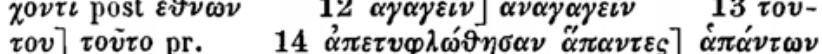

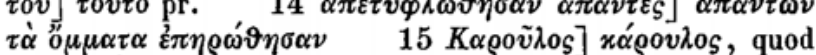
dedi 'P $\tau$ ò] om.

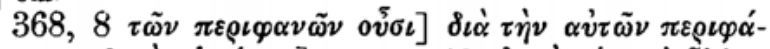

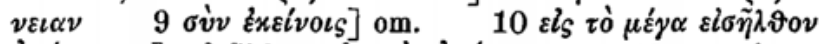

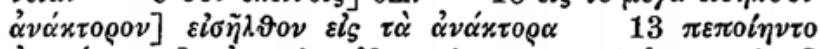

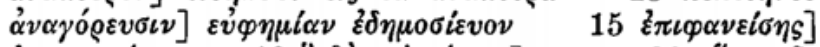

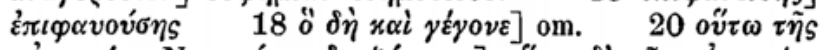

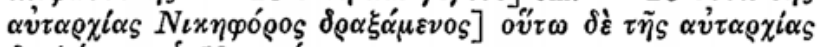

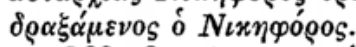

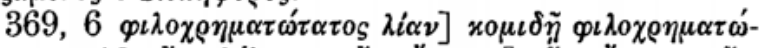

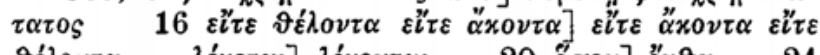

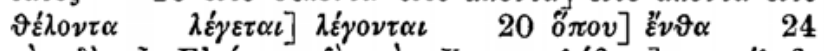

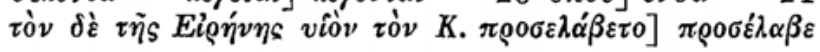




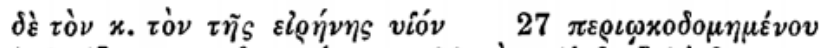

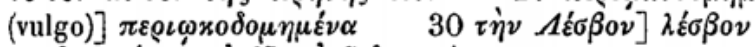

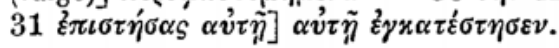

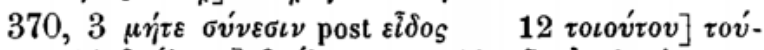

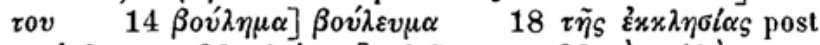
$\gamma \varepsilon v \varepsilon \dot{\sigma} \sigma \alpha \iota \quad 20 \pi \lambda \varepsilon \dot{i} \sigma \tau \sigma v] \pi \lambda \varepsilon \tilde{\imath} \sigma \tau o \nu \quad 28 \tau \dot{\alpha} \pi 0 \lambda \lambda \dot{\alpha} \pi \alpha \rho \alpha-$ $\lambda \iota \pi \dot{\omega} \nu] \pi \alpha \rho \alpha \lambda \iota \pi \dot{\omega} \nu \tau \dot{\alpha} \pi 0 \lambda \lambda \dot{\alpha}$.

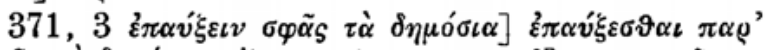

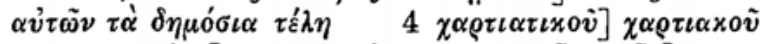

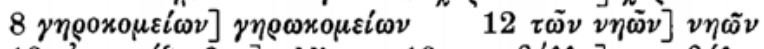
$\left.13 \alpha \nu \alpha \gamma x \alpha^{\prime} \xi \varepsilon \sigma \vartheta \alpha \iota\right]$ addit $\left.18 x \alpha \tau \alpha \beta \alpha^{\prime} \lambda \lambda \eta\right] x \alpha \tau \alpha \beta \alpha^{\prime} \lambda o \iota$

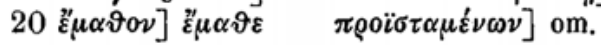

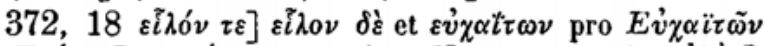

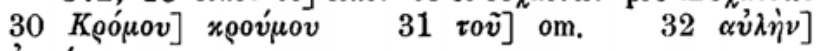

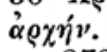

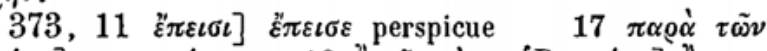

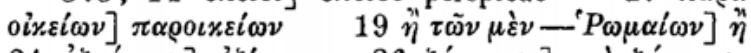

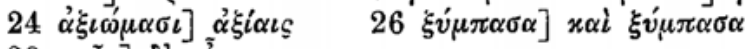

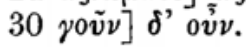

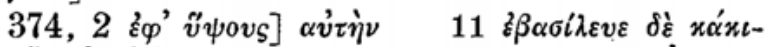

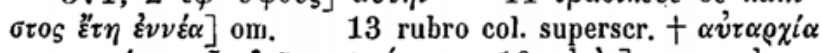

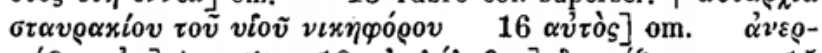

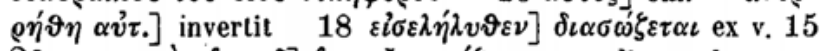

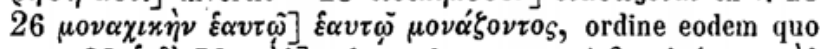

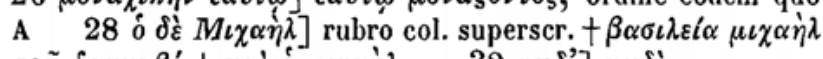
$\tau 0 \tilde{v} \hat{\varrho} \alpha \gamma \gamma \alpha \beta \dot{\varepsilon}+x \alpha i$ o $\mu \iota \chi \alpha \dot{\eta} \lambda \quad 32 \mu \eta \delta$ '] $\mu \eta \delta \dot{\varepsilon}$.

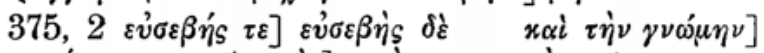

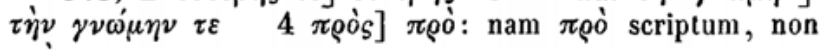

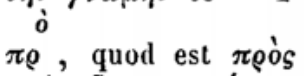

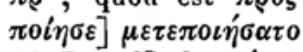

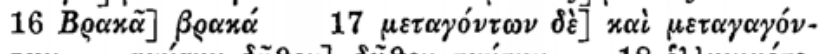

$10 \alpha v i \tilde{\eta}] \hat{\varepsilon} \alpha v \tau \tilde{\eta}, \quad 11, \mu \varepsilon \tau \varepsilon-$ $\tau \omega \nu \tau \alpha v \tau \eta v \delta \tilde{\eta} \vartheta \varepsilon \nu] \delta \tilde{\eta} \vartheta \varepsilon v \tau \alpha v i \tau \nu \quad 18 \varepsilon \lambda \lambda \eta v \iota x o ́ \tau \varepsilon-$

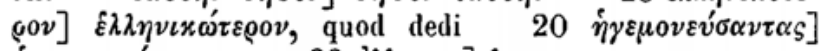
iे

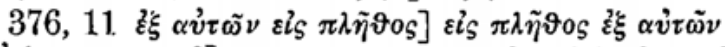

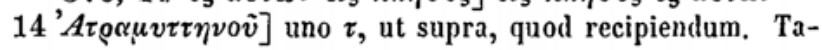


cel Haasius de A $17 \tau \tilde{\omega} \nu \chi \varrho \iota \sigma \tau \omega \nu v ́ \mu \omega \nu] \chi \varrho \iota \sigma \tau \iota \alpha \nu \tilde{\omega} \nu \quad 28$

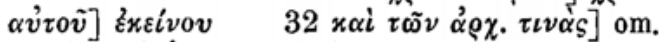

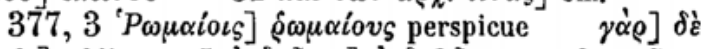

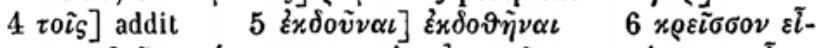

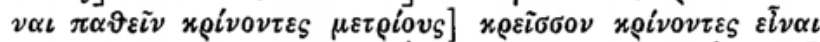

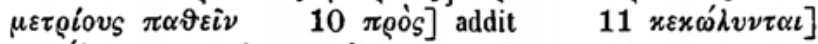

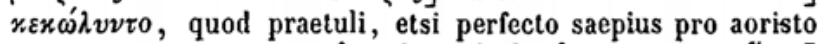

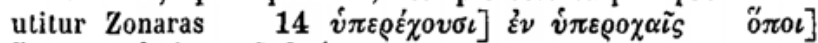

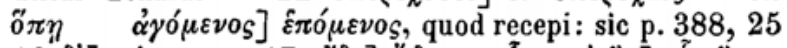

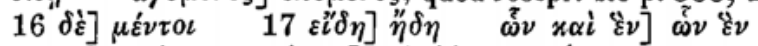

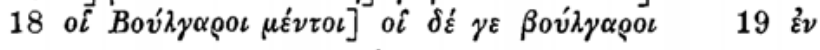

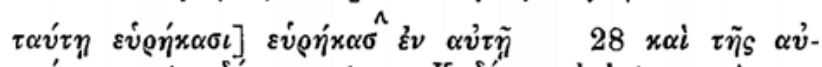

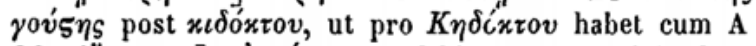

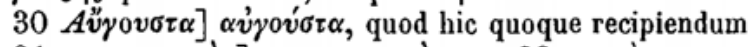

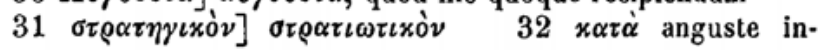
sertum.

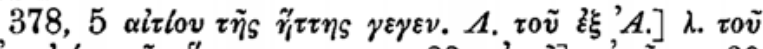

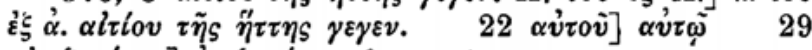

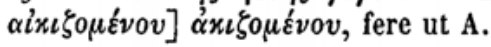

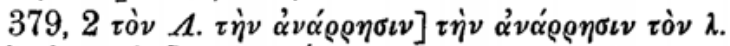

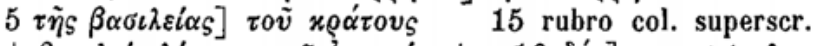

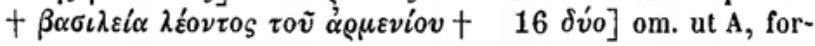
tasse $\beta^{\prime} \beta \alpha \sigma$. volentes. Sed est in Mon. $\beta \sigma i ̈$, ut pro litteris tribus primis librarius aliud quid antea scripserit, etsi id

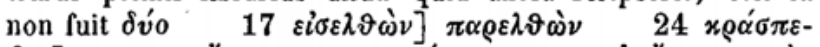

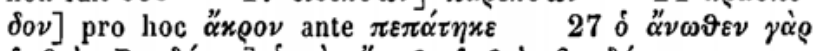

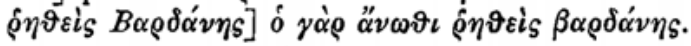

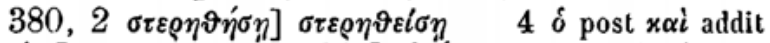

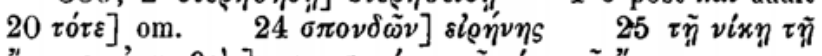

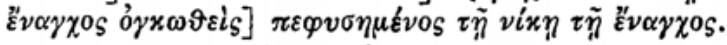

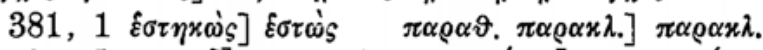

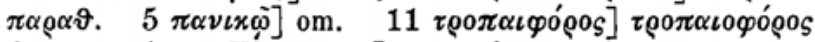

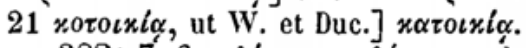

$382,7 \beta \alpha \sigma \iota \lambda \varepsilon_{\alpha} \alpha$ super $\lambda \varepsilon^{\prime} o \nu \tau \alpha$ ead. $\mathrm{m}$.

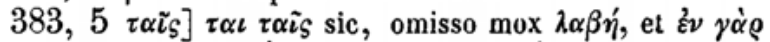

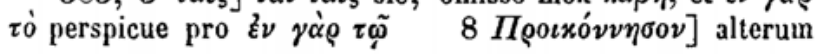


$v$ supra versum ead. $\mathrm{m} . \quad 10$ ov่ $\gamma \alpha \dot{\alpha}-12 \alpha \dot{v} \tau \dot{o} v] \alpha \dot{v} \tau \dot{v} v$

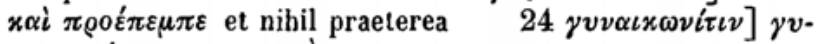
$\nu \alpha \iota x \omega \nu i \tau \eta v$, servans $\tau \dot{\eta} \nu$.

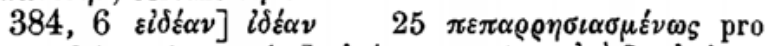

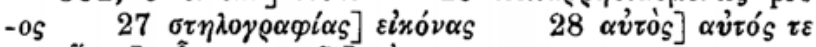

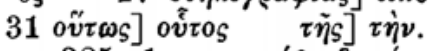

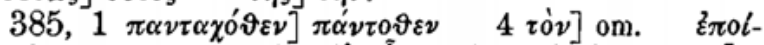

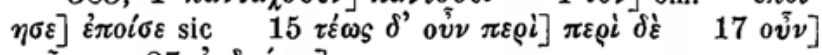

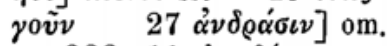

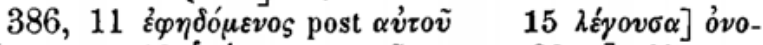
$\mu \alpha^{\prime} \xi_{0 v \sigma \alpha} \quad 16 \dot{\eta} \mu \dot{\varepsilon} \rho \alpha \nu$ ante $\left.\tau 0 \tilde{v} \varphi \varrho . \quad 20 \gamma \varepsilon\right]$ addit

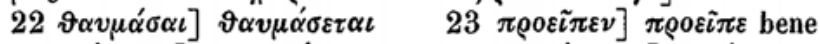

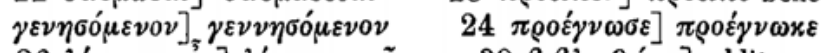

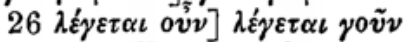

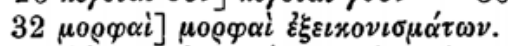

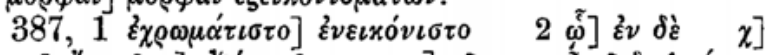

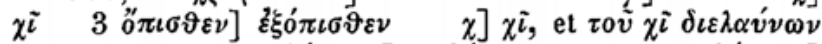
bis ponit $\left.\quad 5 \Sigma \iota \beta v ́ \lambda \lambda \varepsilon \iota \alpha] \sigma \iota \beta v^{\prime} \lambda \lambda \iota \alpha \quad \chi \varrho \eta \sigma \mu \omega \delta \eta^{\prime} \mu \alpha \tau \alpha\right]$

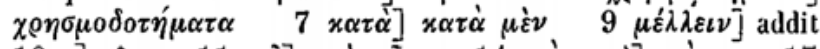

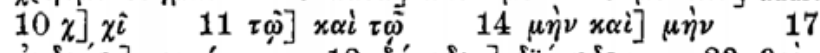

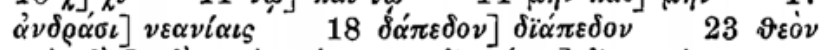

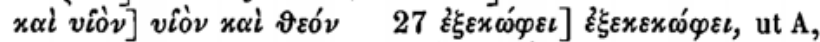
quo de miro codicum vitio pro $\xi^{\xi} \xi x \varepsilon x \omega \varphi q \eta \tau o ~ d i x i$ ad Stepha-

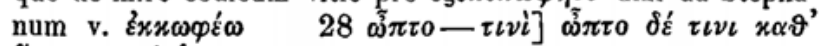

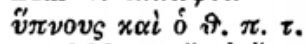

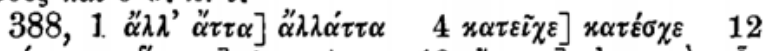

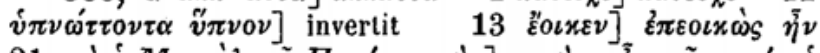

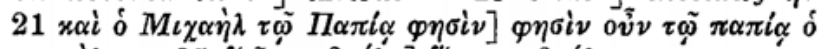

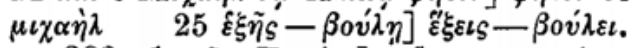

$389,1 \tau \tilde{\omega} v \Pi \alpha \pi i 0 v] \tau \tilde{\omega} \nu \tau 0 \tilde{v} \pi \alpha \pi i o v \quad 3 \mu \varepsilon \tau \alpha \mu-$

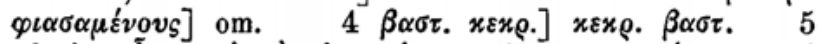

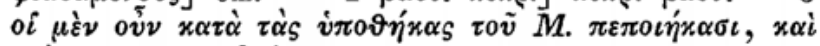
$\tau o \grave{s} \beta \alpha \sigma \iota \lambda \varepsilon i o v \varsigma$ żंź㇒ं $\eta \sigma \alpha \nu$ (sic), ut Ducangius, quum Wolfius idem exhibeat quod ego ex A recepi] of $\mu \dot{\varepsilon} v$ oṽ $v \alpha \tau \dot{\alpha}$

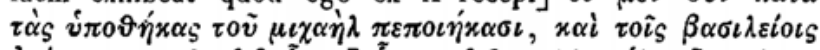

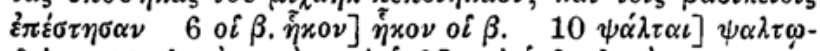

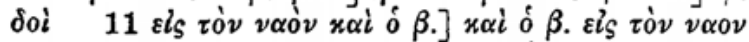

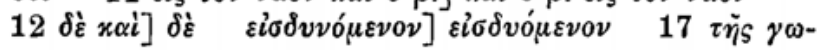


$v i \alpha \varsigma$ post $\mu \varepsilon \lambda \varepsilon \tau \eta \dot{\sigma} \sigma \alpha v \varepsilon \varepsilon_{S}$, omisso $\tau 0 \tilde{v}$ бxótovS $20 \alpha \dot{v} \tau o \tilde{v}$ $\delta \dot{\varepsilon}] \alpha v \tau o \tilde{v}, \quad 22 x \alpha i$ supra v. ead. $\mathrm{m}$. $23 \dot{\alpha} \pi \eta \nu \tilde{\omega}_{S} x \alpha i$

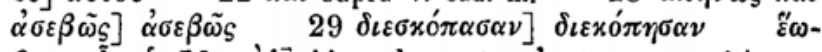
$\vartheta \varepsilon \nu$ ov̉ $v$ o $M \iota \chi \alpha \dot{\eta} \lambda]$ his vulgo ante $\varepsilon l_{S}$ et seqq. positis ru-

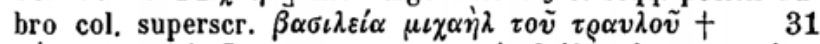

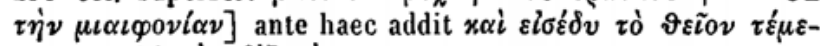
vog $32 \tau \dot{\eta} \nu \delta \dot{\varepsilon}] \tau \dot{\eta} \nu$.

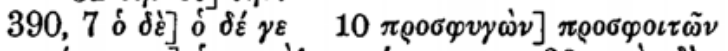

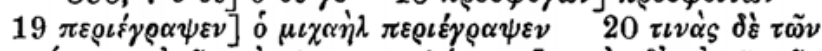

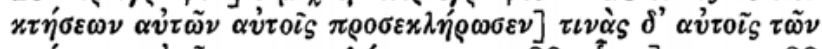

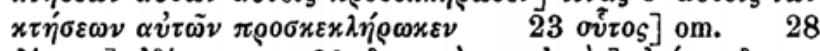

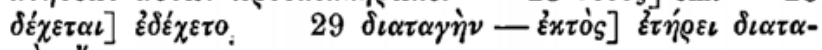

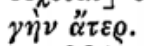

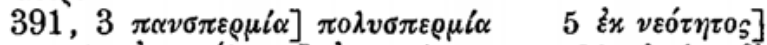

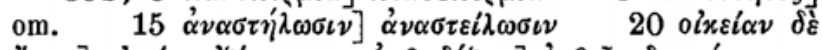

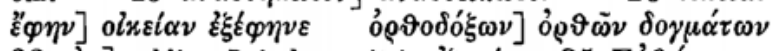

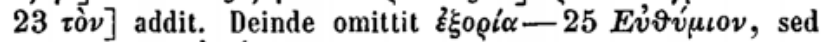
post prius Ev่v์́uıov pallidissimo atramento asteriscus positus supra versum, index lacunae nusquam suppletae $\quad 30$

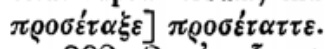

$392,2$ ovx $\varepsilon \tilde{\imath} \nu \alpha \iota \quad \delta.] \delta . \mu \grave{\eta} \varepsilon \hat{l} \nu \alpha \iota \quad 4 \tau \tilde{\omega} \delta \dot{\varepsilon}$ 'Iov́ $\delta \alpha]$

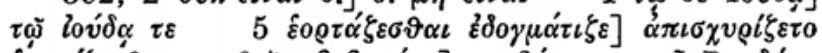

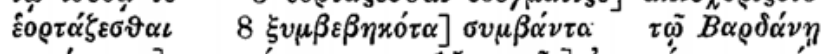

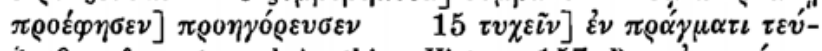

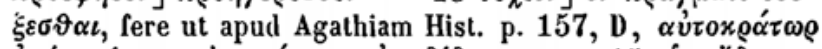

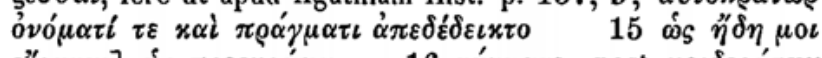

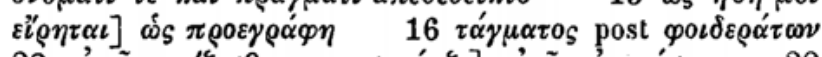

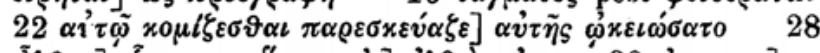

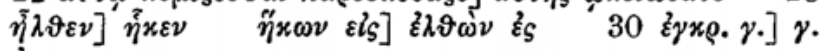
Eิyxo.

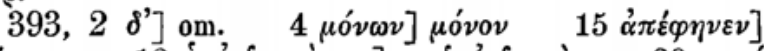

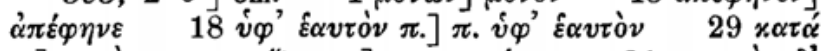

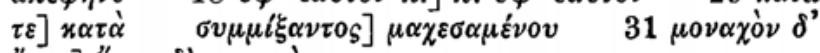

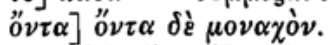

$394,5$ x $\alpha i]$ x $\alpha i$ x $\alpha \tau \grave{\alpha}$

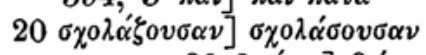
$\gamma \nu v \nu \tau \alpha \iota$

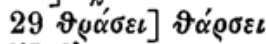

$\left.395,7 \delta_{\varepsilon}\right] \delta^{\prime}$, quod dedi

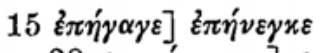

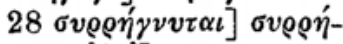
$\left.32 \dot{\alpha} \lambda \lambda^{\prime}\right]$ om.

$\left.13 \pi \alpha \rho \alpha \delta \varepsilon \delta \delta^{\prime} * \alpha \sigma \iota\right] x \alpha \tau \varepsilon v-$ 


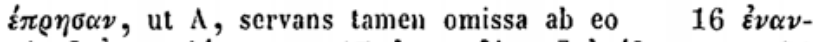

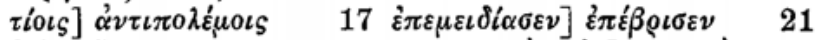

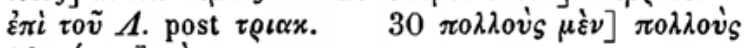

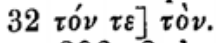

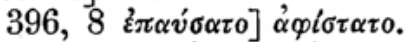

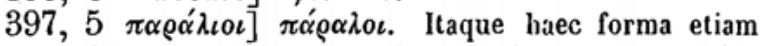
vol. 1, p. 467, A, ex A recipienda, quae in utroque p. 349 ,

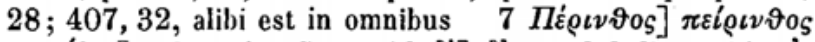
$\left.\left.\pi \alpha^{\prime} \lambda \alpha \iota\right] \tau 0 \tilde{i}_{S} \pi \alpha \lambda \alpha \iota 0 \tilde{\iota}_{S} \quad 10 \delta \dot{\varepsilon}\right] \delta^{\prime}$, quod dedi $14 \alpha \dot{v}$ -

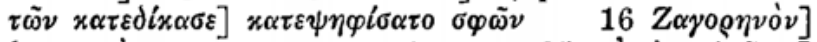

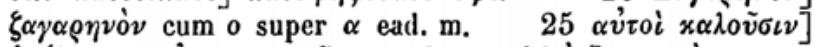

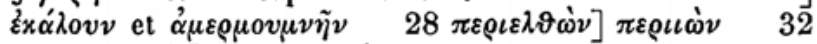
$\pi \alpha \rho \alpha \lambda \varepsilon \iota \varphi \vartheta \varepsilon i \varsigma] \pi \varepsilon \rho \iota \lambda \varepsilon \iota \varphi \vartheta \varepsilon i_{S}$ perspicue, quod recipiendum.

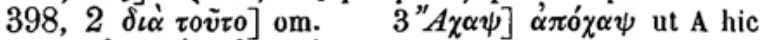

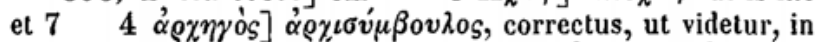

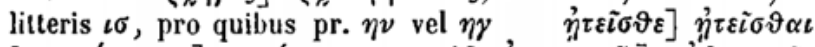

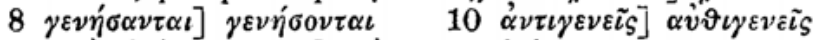

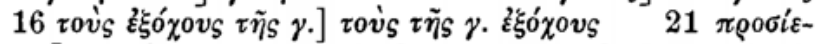
$\tau \alpha \iota] \pi \rho o \sigma i \varepsilon \tau o$, quod recipiendum, quum verba haec omiserit A, nullanque fidem habeat ed. Wolf. contra Mon. $22 \xi \xi$

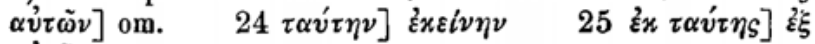
$\alpha \dot{v} \tau \tilde{\eta} \varsigma$.

$\left.399,7 K \alpha \sigma \sigma t \mu \alpha \tau \tilde{\alpha}_{S}\right] \quad x \alpha \sigma \sigma v \mu \alpha \tau \tilde{\alpha}_{S} \quad 18 \tau 0 \lambda \mu \eta \sigma \alpha \dot{\nu}$ -

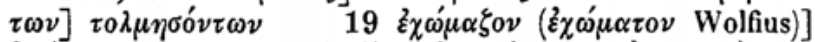

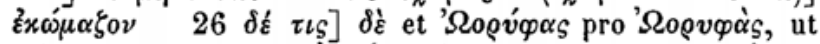
infra vol. 4, p. 31, 22, 'O $\varrho v ́ \varphi \alpha_{S}$ Cellr. p. 511, D $29 \tau \dot{\eta} \nu-$

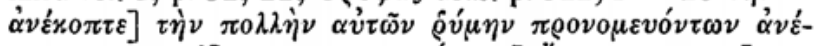

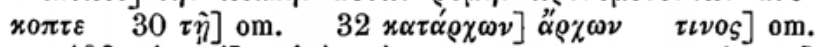
$400,1$ x $\alpha i] x \alpha i \mid x \alpha i$ inter duo versus $\tau i \vartheta \varepsilon \tau \alpha i]$

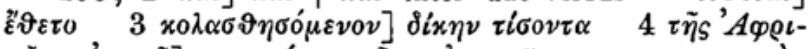

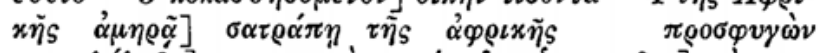
$\left.\left.\pi \varrho 0 \sigma \varepsilon \lambda \eta^{\prime} \lambda v \vartheta \varepsilon\right] \pi \varrho 0 \sigma \varphi v \gamma \omega \dot{\omega} \nu \alpha \alpha i, o i-v i \pi \iota \sigma \chi v \varepsilon i \tau 0\right] \tau \dot{\eta} \nu \sigma \iota-$

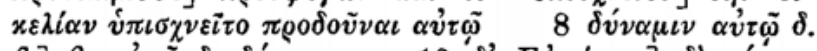

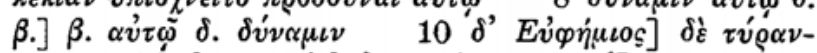

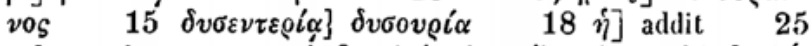

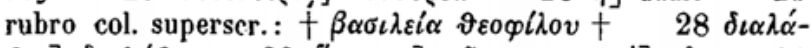
$\left.\left.\vartheta 0 \iota] \delta \iota \alpha \lambda \alpha^{\prime} \vartheta \eta \quad 29 \ddot{\alpha} \pi \alpha \sigma \alpha \nu\right] \pi \tilde{\alpha} \sigma \alpha \nu \quad \pi \varepsilon \rho i\right] \varepsilon i \zeta \quad 30$ $\pi \lambda \eta \varrho о \tilde{v} \nu] \pi \lambda \eta \varrho \circ \tilde{v} \nu \tau \alpha$. 


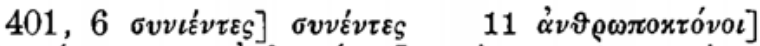

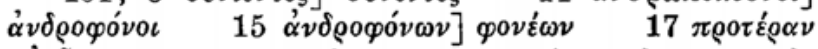
$\alpha \dot{v} r \tilde{\eta}_{S}$ extremo versu ead. manu in margine eodemque modo initio sequentis $\mu$ ov $\eta^{2} \varepsilon^{2} \nu \tau \tilde{\eta}$ ead. manu; ut utraque versum excedant $26 \alpha v \tau \dot{0}] \alpha v i \tilde{\omega} \quad 27 \tilde{\eta} \lambda \vartheta \varepsilon v] \tilde{\eta} \lambda \vartheta \varepsilon$ bene, et 32

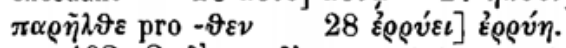

$402,2 \delta$ 'pro $\delta \dot{\varepsilon}$, quod dedi $11 \hat{\varepsilon} \alpha v \tau \tilde{\eta}]$ ह $\alpha v \tau \tilde{\omega}$

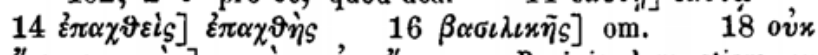

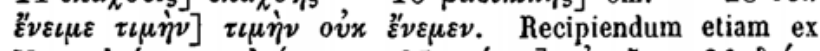

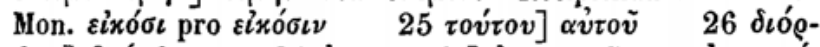

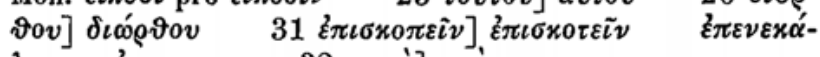
$\lambda \varepsilon \iota$ pro $\varepsilon \pi \varepsilon v \varepsilon \gamma$ - $\quad 32 \pi \alpha \rho \dot{\alpha}] \pi \varrho o_{s}$.

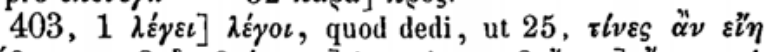

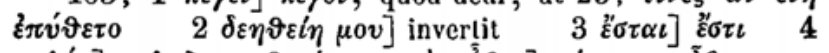

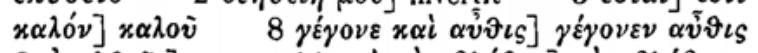

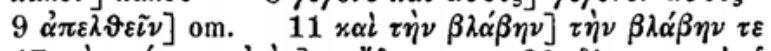

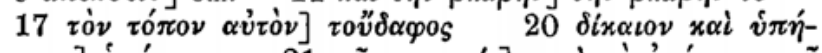

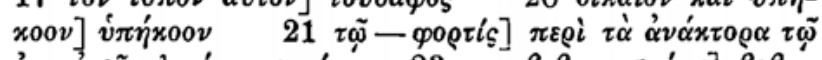

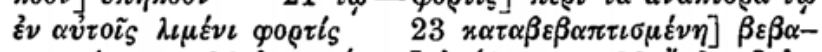

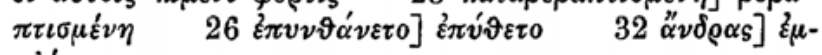
$\pi \lambda \varepsilon_{0} \nu \tau \alpha_{\text {S. }}$.

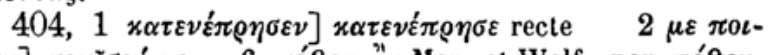

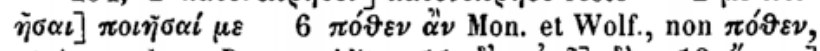
ut A, quod ap. Duc. excidit $\left.\left.11 \delta^{\prime} \alpha \hat{v} \tau \tilde{\eta}\right] \quad \delta \dot{\varepsilon} 13{ }^{\prime}{ }^{\prime \prime} \mu \varphi \omega\right]$

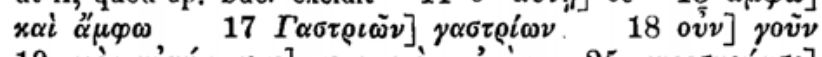

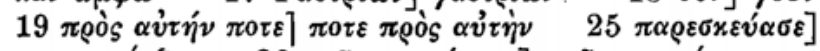

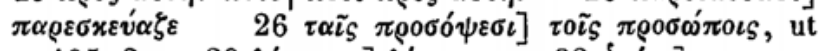

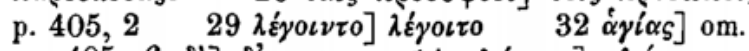

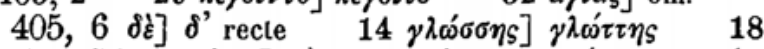

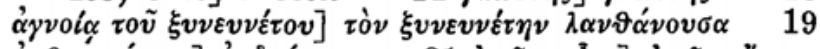

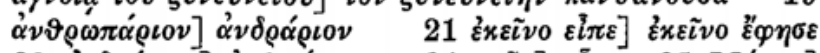

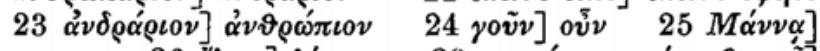

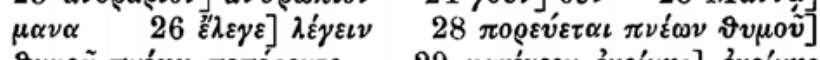

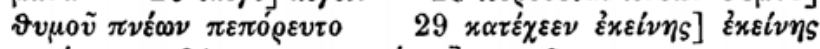

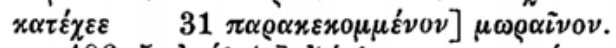

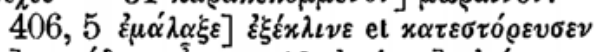

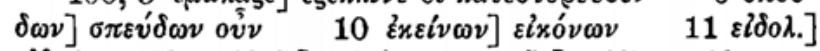
$\left.\left.\varepsilon i \delta \omega \lambda . \quad 18 \pi 0 \lambda \lambda_{0} \tilde{i}_{S}\right] \pi \lambda \varepsilon i \sigma \tau o \iota \varsigma \quad \tau \tilde{\omega} \nu\right]$ addit $19 \pi \varepsilon \mu-$ 
$\varphi \vartheta \varepsilon ́ v \tau \iota] i \omega \alpha ́ v \nu \eta$ litterae desunt sive propter rasuram sive propter foramen

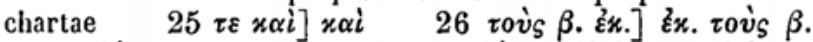

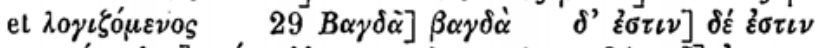

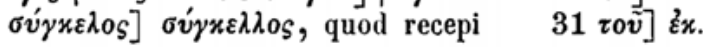

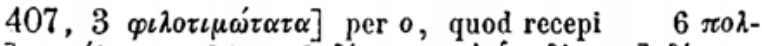

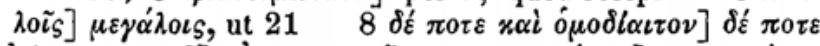

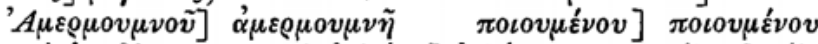

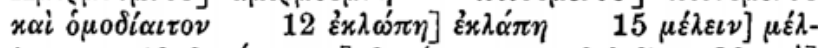

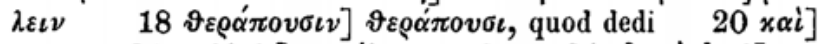

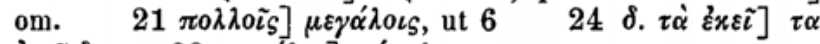

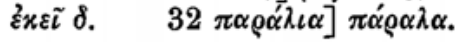

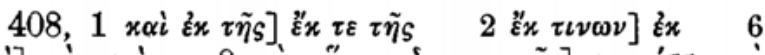

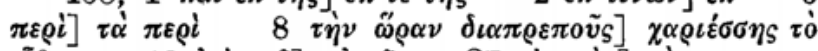

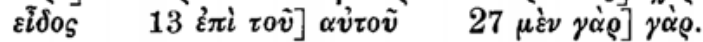

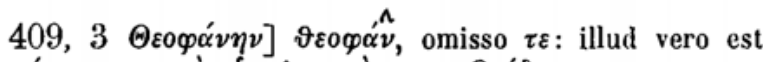

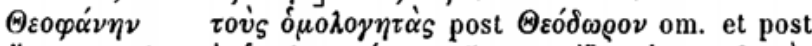

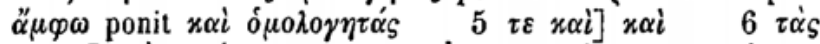

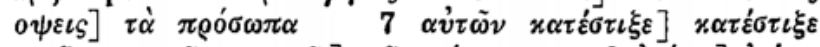

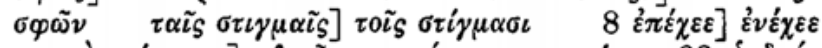

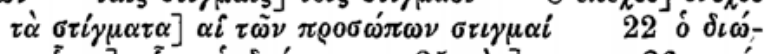

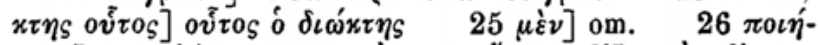

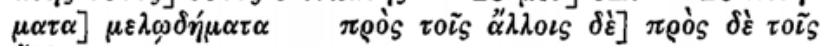
"ैh גoเs.

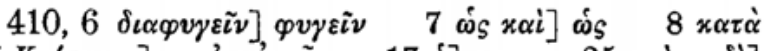

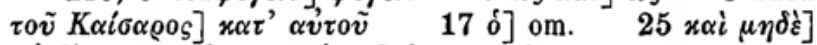

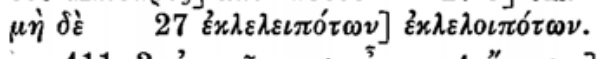

411, $3 \stackrel{\alpha}{\alpha} \mu \varphi \circ \tilde{\iota} \nu$ post ov $\stackrel{s}{*} \quad 4 \stackrel{\alpha}{\alpha} \gamma \chi \iota \tau \tau]$ om. et in marg. ead. m. $7 \sigma \tau \rho \alpha \tau \eta \gamma \tilde{\omega} \nu] \sigma \alpha \tau \rho \alpha \pi \omega \nu$, sed pr., ut videtur, $\sigma \tau \varrho \alpha \tau \varepsilon \iota \tilde{\omega} \nu \cdot$ nam $\sigma \alpha$ est in rasura et litterae inter $\alpha$ et $\nu$

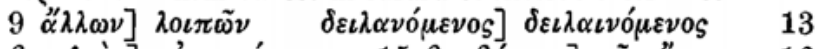

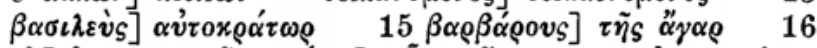

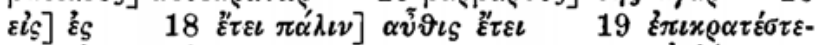

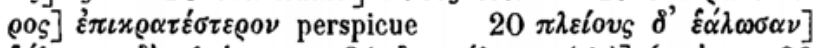

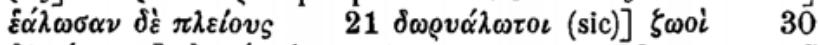

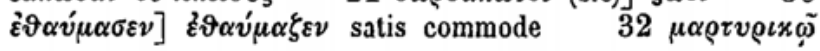




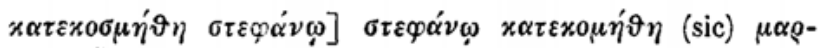

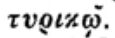

$4 \hat{1} 2,1 \hat{\omega} \nu] \gamma \varepsilon \gamma o \nu \omega \dot{s}, 2 \tau \hat{\eta}] \tau \tilde{\eta}$ in rasura paullo am-

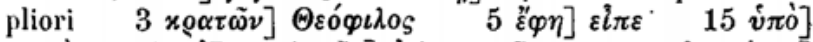

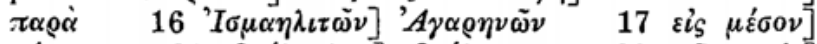

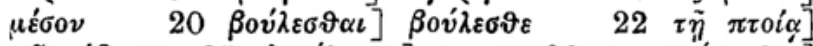

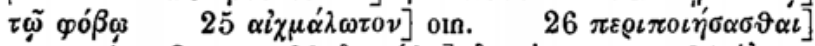

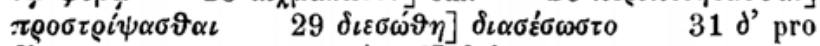
$\delta \dot{\varepsilon}$, quod dedi $32 x \alpha \tau \dot{\alpha} \tau o v]$ हों $\tau i \tilde{\omega}$.

$\left.413,1 \delta_{\iota \varepsilon} \beta \alpha \lambda_{0 \nu}\right] \delta\left\llcorner\varepsilon \dot{\beta} \beta \lambda \lambda_{0 \nu} \quad 7 . \vartheta \varrho \eta \sigma x \varepsilon i^{\prime} \alpha \nu\right]$ bis

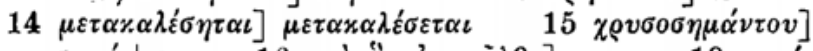

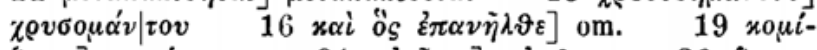

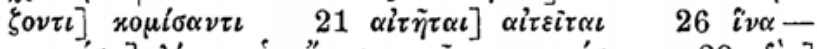

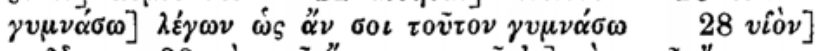

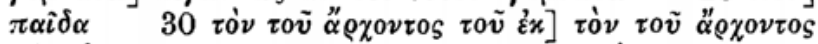
$\tau \tilde{\omega} \nu \varepsilon x$, unde recipiendum certe $\tau \tilde{\omega} \nu$ pro $\tau \hat{o} v$, quod malis etiam 24, ubi $\tau \hat{\nu} \nu$ praebuit A pro $\tau \tilde{\omega} v$ : nam semper' $A \gamma \alpha \varrho \eta$ voi est cum articulo et óhíov male A pro ódí $\omega \nu 31$.

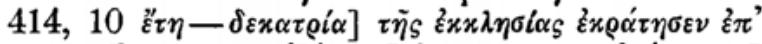

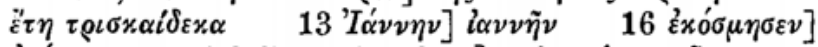

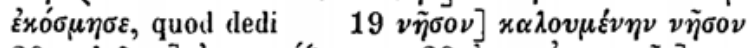

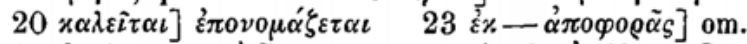

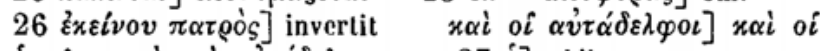

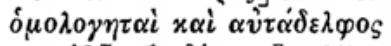

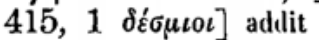
27 ó] addit.

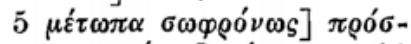

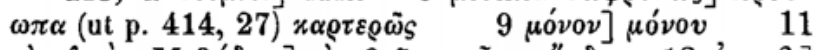

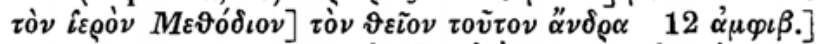

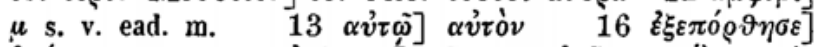

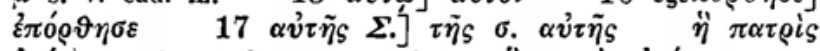
$\varepsilon^{2} \tau v^{\prime} \gamma \mid \chi \alpha \nu \varepsilon$ inter duo versus ita ut $\ddot{\eta} \pi \alpha \tau \varrho i s \varepsilon \varepsilon^{2} \tau v^{\prime} \gamma-$ versum excedat in fine eius positum, non $\chi \alpha \nu \varepsilon$ in sequentis initio $25 \delta \grave{\varepsilon} x \alpha i] \delta \dot{\varepsilon} \quad 26 \mu \grave{\eta} x \alpha i] \mu \grave{\eta}, \quad\{\alpha v \tau \dot{v} \nu] \alpha \hat{v} \tau \dot{\nu} \nu \quad 27$

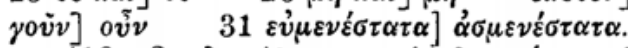

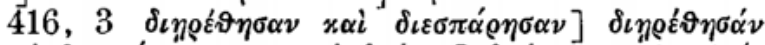

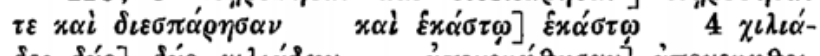

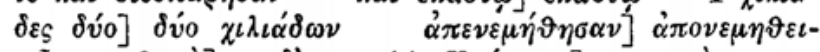

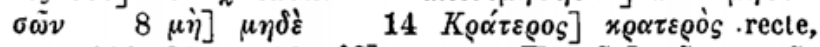
ut p. 411,31 $19 v \hat{i} \hat{\omega}]$ om. $\Pi \varepsilon \varepsilon \sigma \tilde{\omega} \nu] \tau \tilde{\omega} \nu \pi \varepsilon \rho \sigma \tilde{\omega} \nu$ 


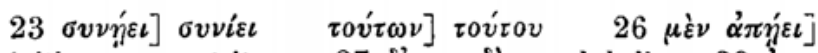
initio versus extrita $27 \delta$ 'pro $\delta \dot{\varepsilon}$, quod dedi $28 \dot{\varepsilon} \pi l$ -

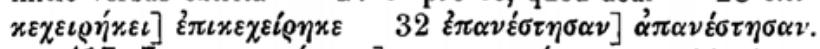

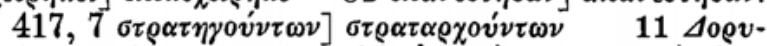

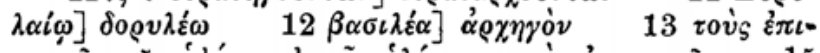

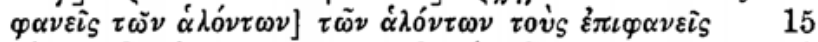

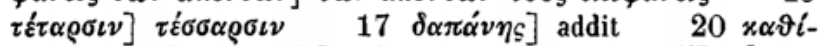

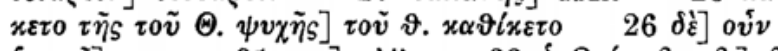

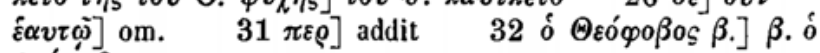
จะó́oßos.

$418,1 \alpha \dot{v} \tau \dot{\nu} \nu]$ om. $\quad 5 \dot{\omega} \varsigma$ ov $\nu$ extrita in fine versus

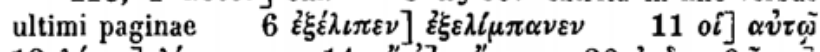

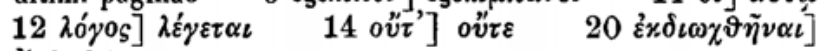
$\xi \xi \xi \lambda \alpha \vartheta \tilde{\eta} v \alpha \iota$.

Vol. 4, p. 1, 1 rubro superscr. $\beta \alpha \sigma \iota \lambda \varepsilon i \alpha \mu \iota \chi \alpha \dot{\eta} \lambda$, $\tau 0 \tilde{v}$

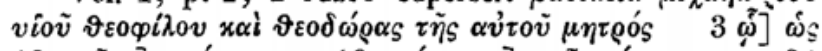

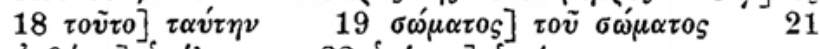

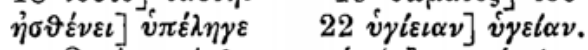

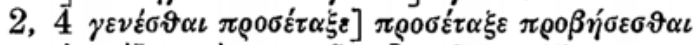

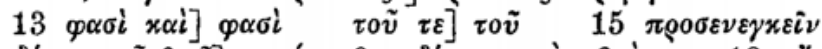

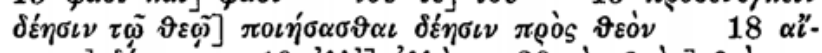

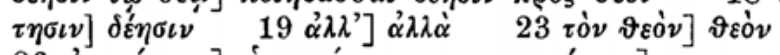

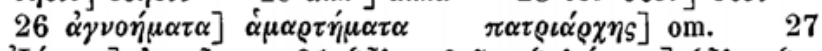

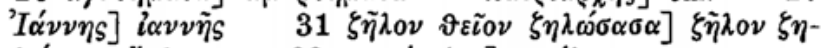

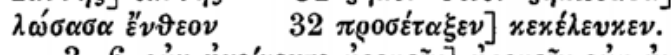

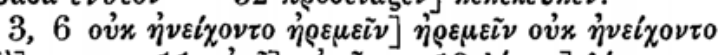

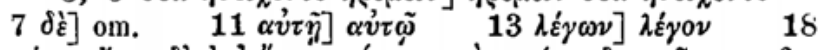

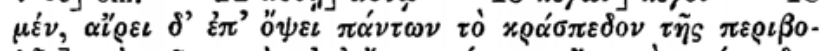

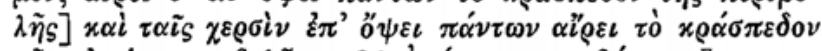

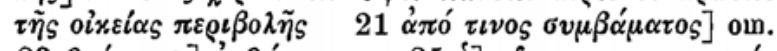

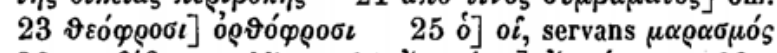

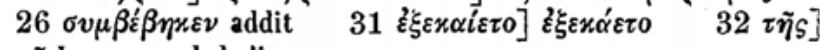
$\tau \tilde{\eta}$ bene, quod dedi.

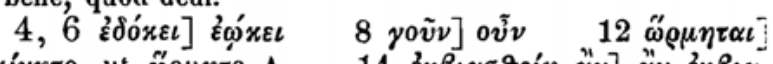

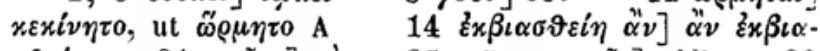

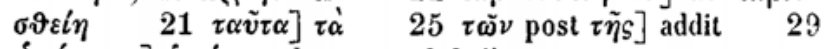

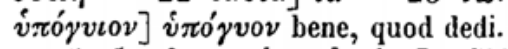

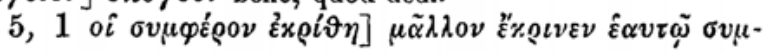




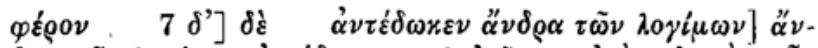

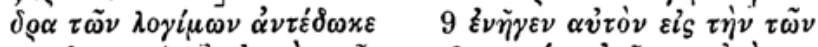

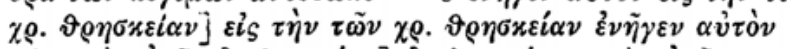

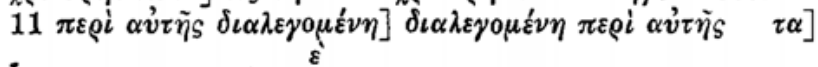

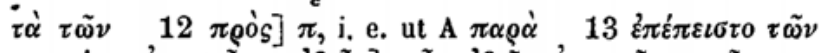

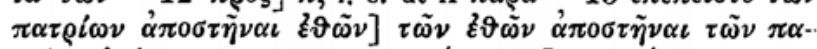


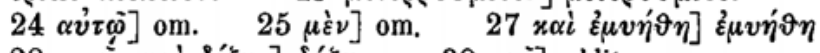

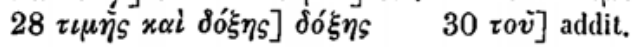

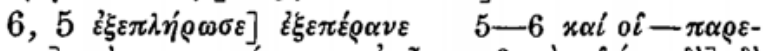

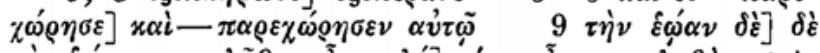

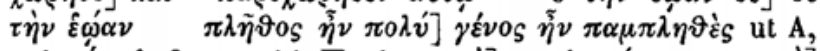
qui $\left.\left.\pi \alpha^{\prime} \mu \pi \lambda \eta \vartheta \varepsilon, \quad 11 \Pi \alpha v \lambda \iota x \iota \alpha \nu 0 i\right] \pi \alpha v \lambda \iota x i \alpha^{\prime} \nu 0 \iota \quad \pi \alpha \rho \dot{\alpha}\right]$

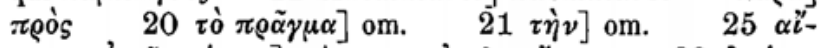

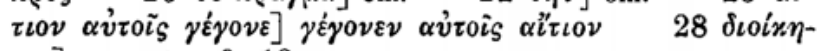
$\sigma \iota \nu]$ om., ut p. 9,18 .

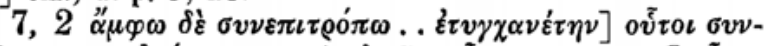

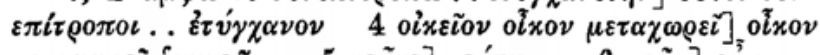

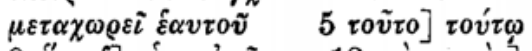

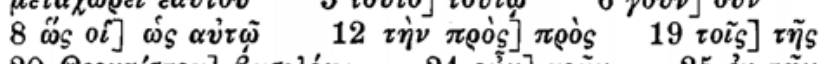

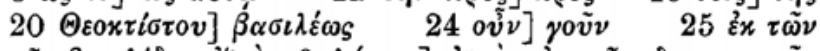

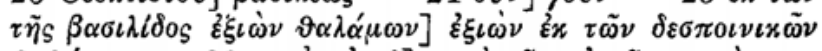

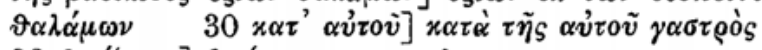
$\left.32 \vartheta v \eta^{\prime} \xi o v \tau \iota\right] \vartheta v \eta^{\prime} \sigma x o v \tau \iota$, recte fortasse.

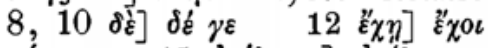
$13 \vartheta \eta \sigma \alpha v \rho \tilde{\omega} \nu]$ $\chi \rho \eta \mu \alpha ́ \tau \omega \nu$

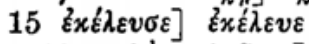

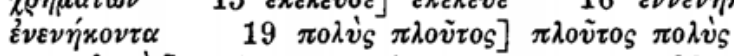
$\pi \alpha \nu \tau o \delta \alpha \pi \dot{o} s]$ o alterum pr. fuisse videtur $\eta \quad 22 \alpha v \tau \alpha \rho-$ $\left.\left.\chi i \alpha_{S}\right] \beta \alpha \sigma \iota \lambda \varepsilon i \alpha_{S} \quad 31 \delta \dot{\delta}\right] \delta^{\prime}$.

$\left.9,6 \delta \beta \alpha \sigma \iota \lambda \varepsilon v_{S}\right] \delta \quad \mu \iota \chi \alpha \dot{\eta} \lambda$

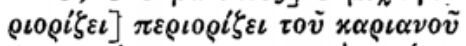
$16 \mu 0 \nu \dot{\nu} \nu 15$ post $\pi \rho o_{S} \tau \dot{\eta} \nu$

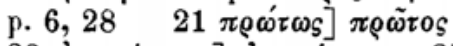

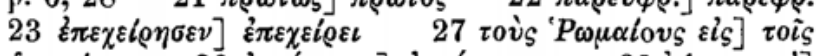

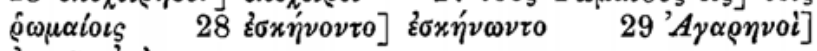

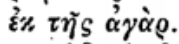

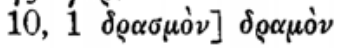

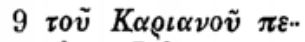

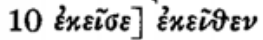

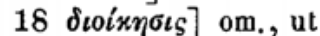
$22 \pi \alpha \rho \varepsilon v \varphi \varrho.] \pi \alpha \varrho \varepsilon \varphi \rho$.

CX 


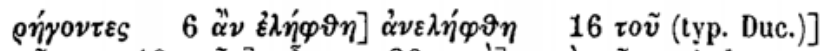

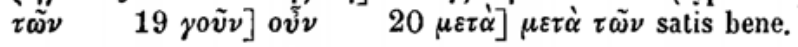

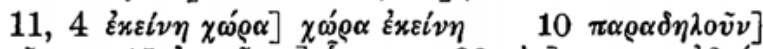

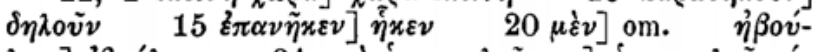

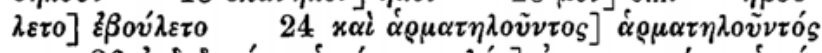

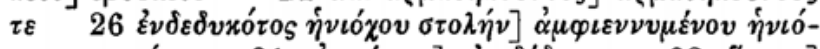

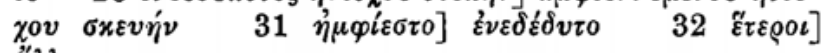

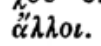

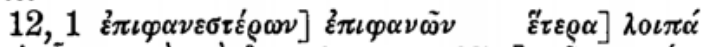

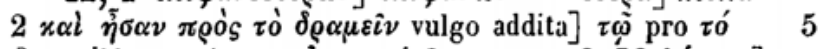

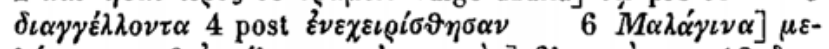

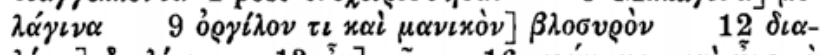

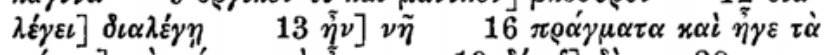

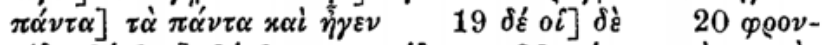

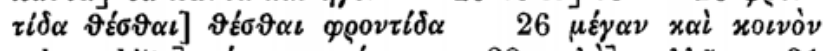

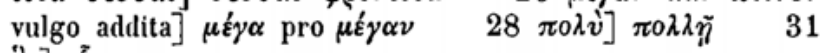
ös] $\tilde{\sigma}^{\tau}$.

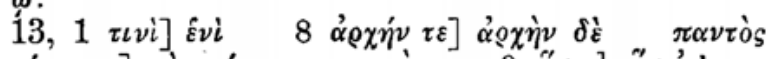

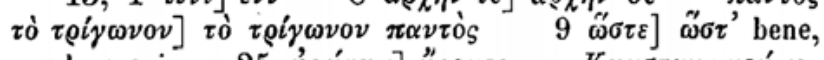

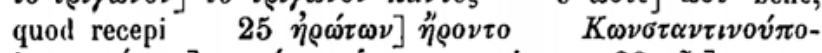

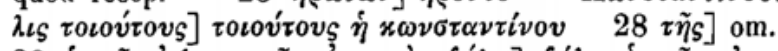

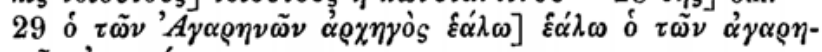
$\nu \tilde{\omega} \nu \dot{\alpha} \varrho \chi \eta \gamma o ́ s$.

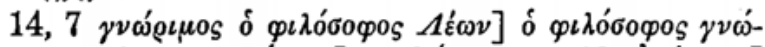

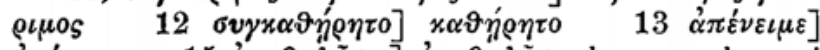
$\alpha \pi \varepsilon v \varepsilon \mu \varepsilon \quad 15 \dot{\alpha} \nu \alpha \vartheta \alpha \hat{\lambda} \sigma \alpha \iota] \dot{\alpha} \nu \alpha \vartheta \eta \hat{\eta} \tilde{\eta} \sigma \alpha \iota$ bene, quod recepi

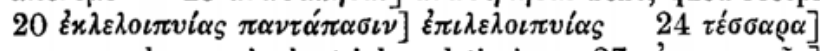
om. cum lacuna duplo triplove latiori $27 \alpha \pi 0 \gamma \varepsilon v \iota x \omega \tilde{\omega} \nu]$

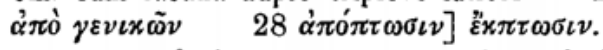

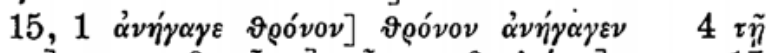

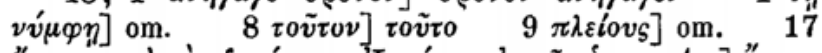

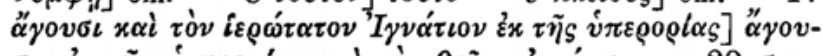

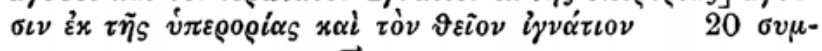

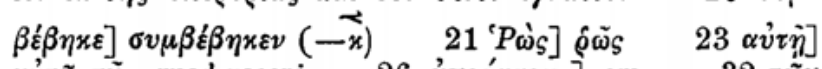

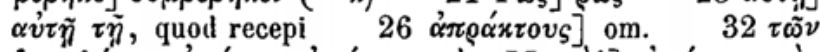

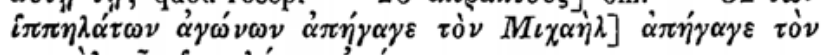
$\mu \iota \chi \alpha \dot{\eta} \lambda \tau \tilde{\omega} \nu i \pi \pi \eta \lambda \dot{\alpha} \tau \omega \nu \alpha \dot{\alpha} \omega \dot{v} \omega \nu$. 


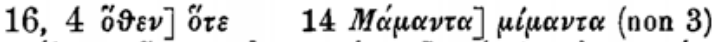

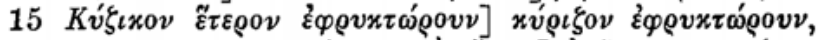

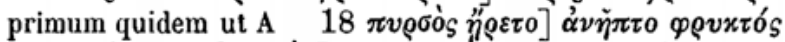

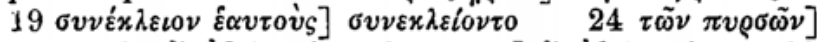

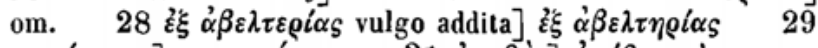

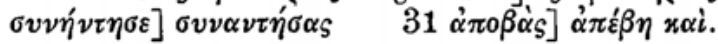

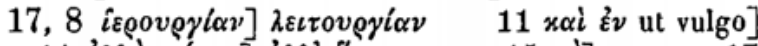

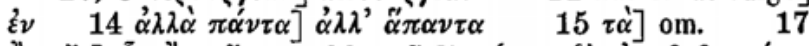

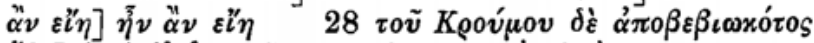

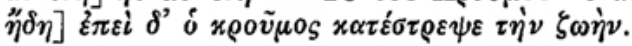

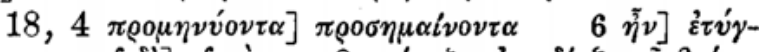

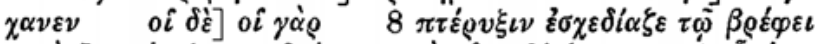

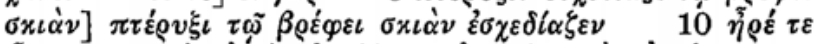

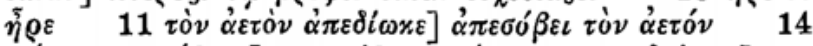

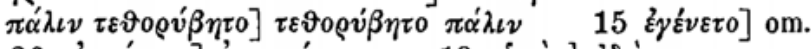

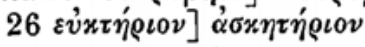

$19 \varepsilon \dot{v} \varrho \omega \dot{\nu}] i \delta \omega \dot{\nu}$.

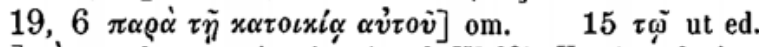
Duc.] $\tau \dot{o}$, quod quum sit etiam in ed. Wolfii, Haasium fugisse videtur in A $16 \chi \varrho \eta \sigma \mu \omega ́ \delta \eta \mu \alpha \bar{\jmath} \chi \varrho \eta \sigma \mu o \delta o ́ \tau \eta \mu \alpha, \tau \tilde{\sigma}$

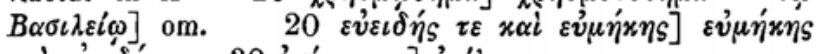

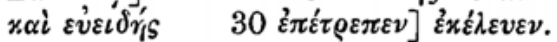

$\left.20,7 \pi \varepsilon \rho \iota x \alpha \lambda \pi \alpha^{\prime} \sigma \alpha_{S}\right] \pi \alpha \rho \alpha x \alpha \lambda \pi \alpha^{\prime} \sigma \alpha_{S}$

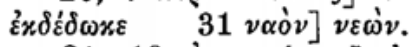

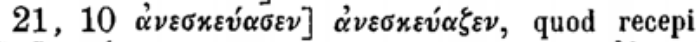

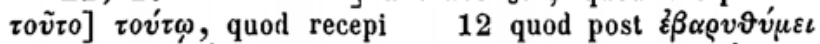

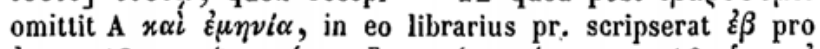

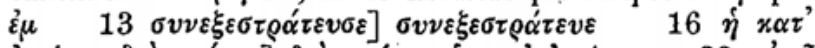

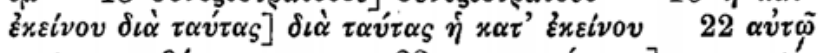

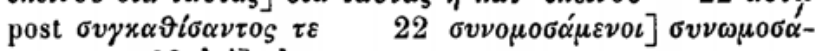

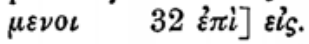

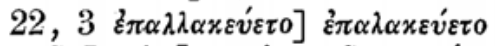

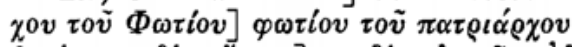

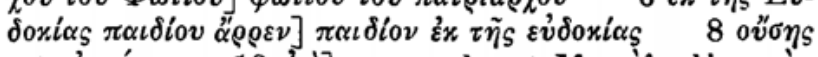

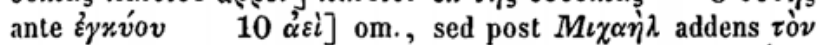

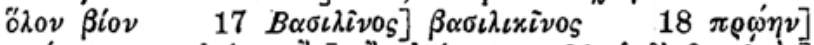

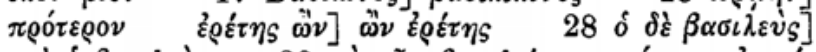

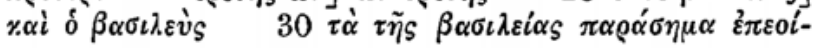




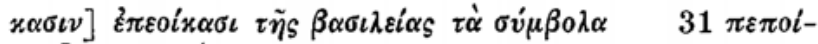
$\eta x \alpha] \pi \varepsilon \pi \circ\llcorner\eta x o ́ \tau \alpha$.

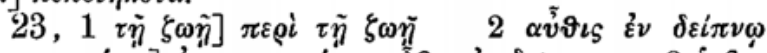

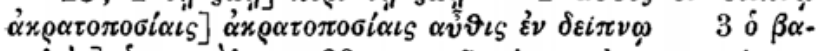
$\sigma v \lambda \varepsilon i \varsigma]$ o $\mu \iota \chi \alpha \dot{\eta} \lambda \quad 23$ ante $\tau \tilde{\omega}$ rubro col. superscriptum

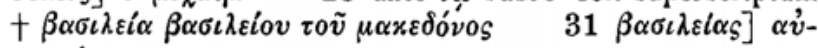
$\tau \alpha \rho \chi i \alpha s$.

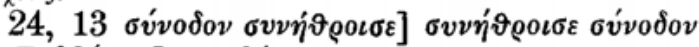

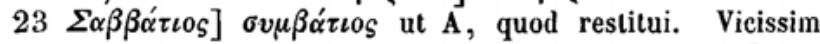
apud Theophanem Chron. p. 306, A, recipiendum ex cod. Paris. $\Sigma \alpha \beta \beta \alpha^{\prime} \tau \iota$ s, ut et apud Zonaram est de eodem homine vol. 2, p. 93, A, et apud Photium cod. 65, p. 29, 39 recte Bekkerus

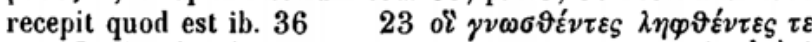

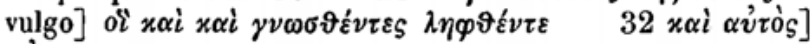

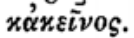

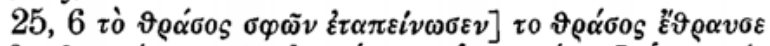

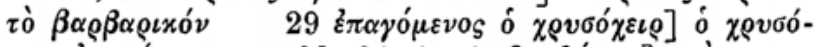

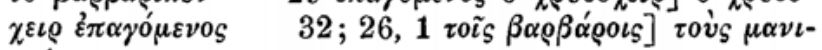

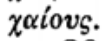

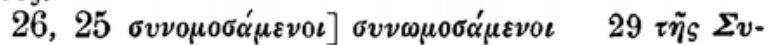
อi $\alpha \varsigma] \sigma v \varrho \iota^{\prime \prime}$ i. e. $\sigma v \varrho i \alpha \iota s$.

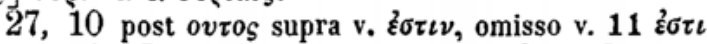

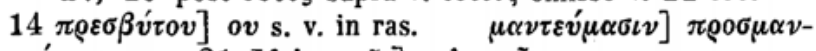
$\tau \varepsilon \dot{v} \mu \alpha \sigma \iota \nu \quad 21 M \varepsilon \lambda \iota \tau \eta \nu \tilde{\eta} s] \mu \varepsilon \lambda_{\iota} \tau \iota \nu \tilde{\eta} \varsigma$.

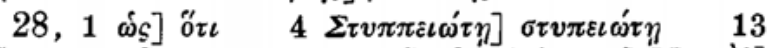

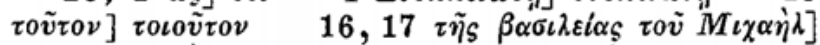

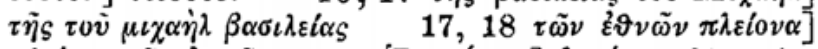

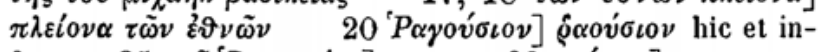

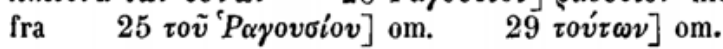

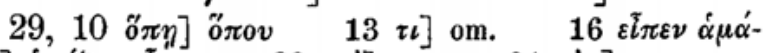

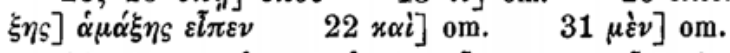

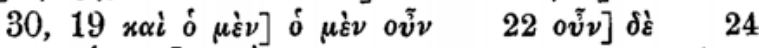
$x \alpha \tau \alpha x \tau \eta \sigma \alpha \dot{\alpha} \mu \varepsilon \nu \varsigma$ ] $x \alpha \tau \dot{\alpha}$ initio versus postea ascriptum videtur

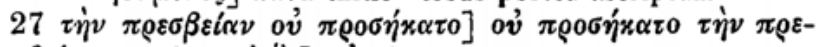

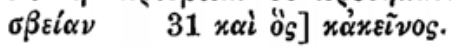

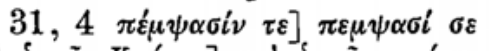

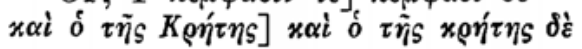
zONARAS $\mathrm{v}$.

$17 \dot{\alpha} \lambda \lambda \dot{\alpha} \mu \dot{\varepsilon} v \tau 0 \iota$

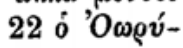
h 


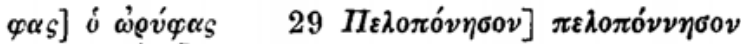
$32 K \varepsilon \gamma \chi \varrho \varepsilon \dot{\omega} \nu]$ ] $\varepsilon \gamma \chi \rho \varepsilon \omega \tilde{\nu}$.

$\left.\left.32,14 N \alpha^{\prime} \varrho \sigma \alpha \nu\right] v \alpha^{\prime} \sigma \alpha \rho 17 \lambda \varepsilon \iota \pi 0 \tau \alpha \dot{x} \tau \alpha \iota\right] \varepsilon \iota$ in ras.

$25 \Pi \varepsilon \lambda$ ก

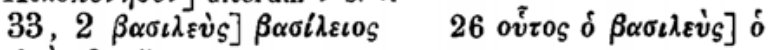
$\beta \alpha \sigma \iota \lambda \varepsilon v \varsigma \beta \alpha \sigma i \lambda_{\varepsilon \iota \iota s}$.

$34,6 \beta \alpha \sigma \iota \lambda \varepsilon \tilde{v} \sigma \iota$ pro $\beta \alpha \sigma \iota \lambda \varepsilon \tilde{v} \sigma \iota v$, quod recepi 18

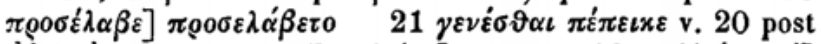

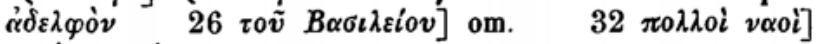
vкoi $\pi 0 \lambda \lambda$ oi.

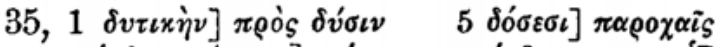

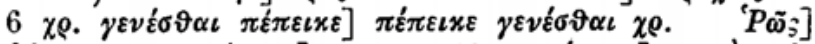

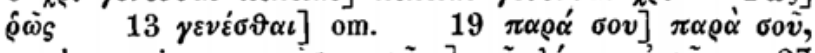

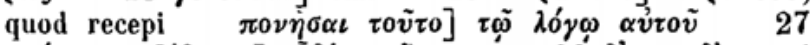

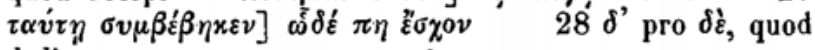
dedi.

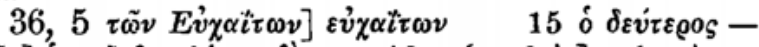

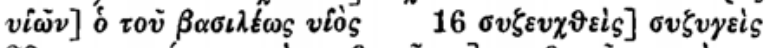

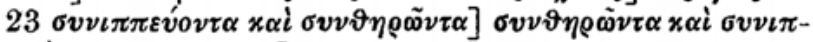
$\pi \varepsilon \dot{v}$ ov $\tau \alpha \quad 32 \sigma o v]$ om.

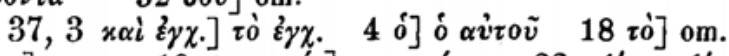

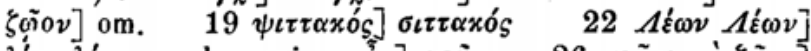

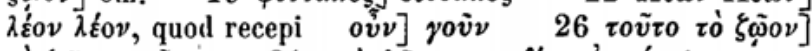

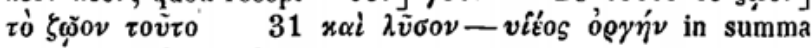
pagina suppleta ead. $m$.

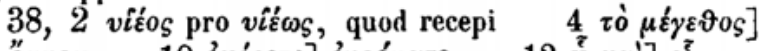

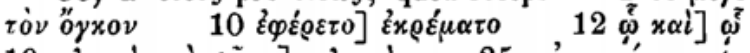
$\left.19 \pi \lambda \eta \gamma \varepsilon i_{S} \tau \dot{\alpha} \sigma \tilde{\omega} \mu \alpha\right] \pi \lambda \eta \gamma \varepsilon i \varsigma \quad 25 \alpha \dot{v} \tau 0 x \rho \alpha \tau \omega \rho$ et seqq.

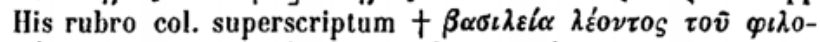

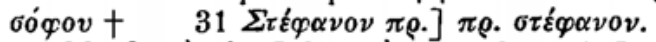

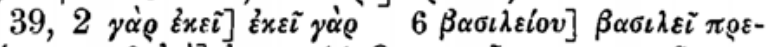

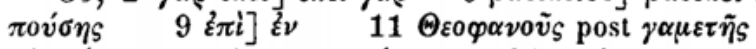

$12 \mu \dot{\alpha} \gamma \iota \sigma \tau \varrho o \nu$ infra versum (paginae ultimum) supplet ead. $m$.

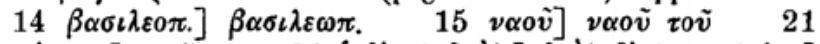

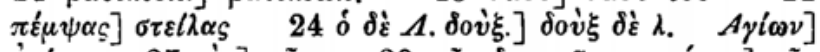

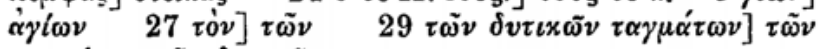

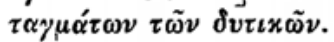

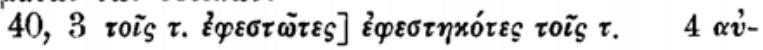




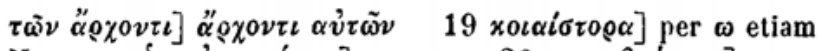

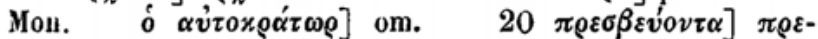

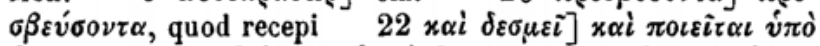

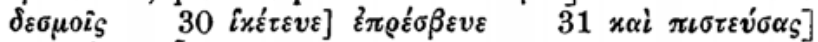
$\pi \iota \sigma \tau \varepsilon \dot{v} \sigma \alpha \varsigma$ ov้v.

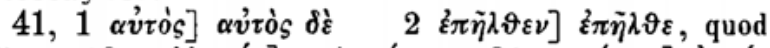

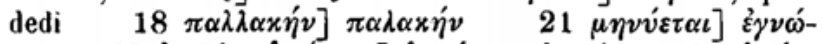

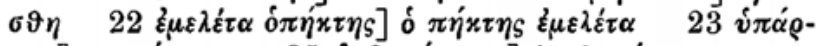
$\chi \omega \nu] \tau v \gamma \chi \alpha \dot{\alpha} \omega \nu \quad 25 \oint \eta \vartheta \eta \sigma o ́ \mu \varepsilon \nu 0 \nu] \lambda \varepsilon \chi \vartheta \eta \sigma o ́ \mu \varepsilon \nu 0 \nu$.

42, $13 \alpha \dot{v} \tau \grave{\eta}] \alpha v \dot{v} \dot{v} v$ rov, ut $\mathrm{A}$, cui congruit quod pro

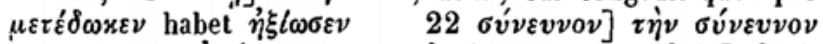

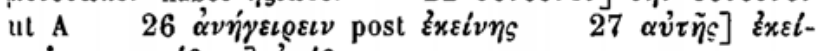
$\nu \eta \dot{\zeta} \quad x \alpha \tau \varepsilon \dot{\varepsilon} \varepsilon \tau 0] \dot{\alpha} \pi \dot{\varepsilon} \vartheta \varepsilon \tau 0$.

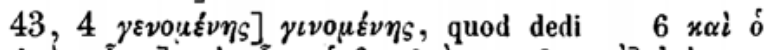

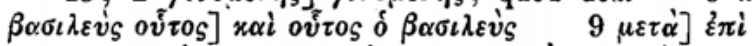

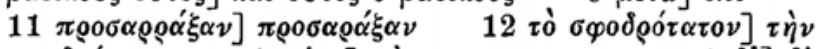
б

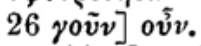

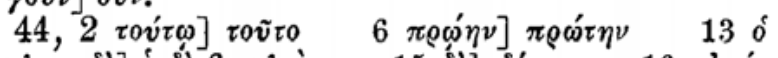

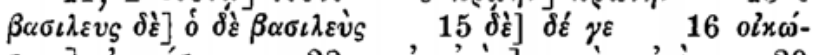

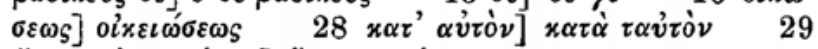

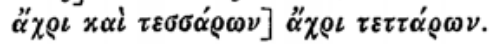

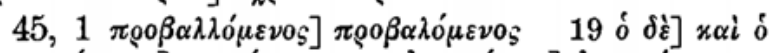

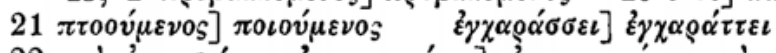

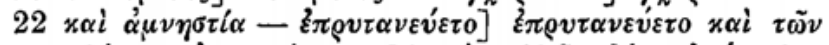

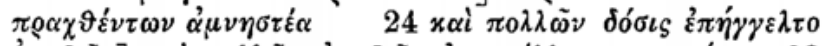

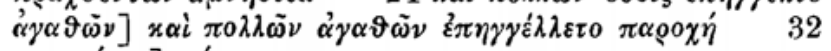

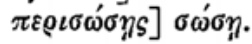

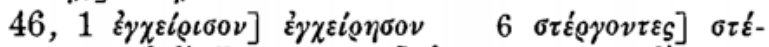

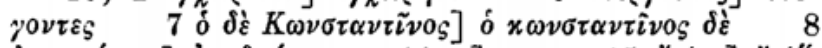

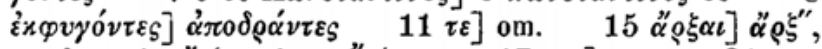
quod nescio $\alpha \rho^{\prime} \xi \iota$ sit an $\left.\alpha \rho \xi \varepsilon \iota \nu 17 \sigma 0 \iota\right]$ om. $21 \chi \omega-$

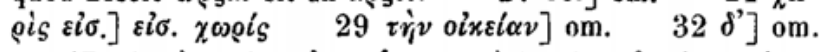

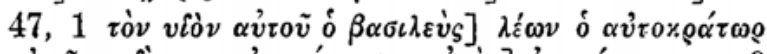

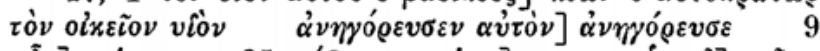

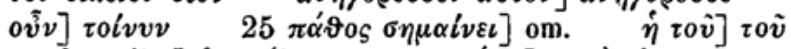

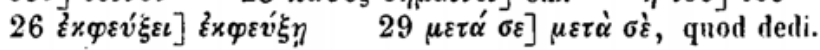




$$
\alpha \dot{v}
$$

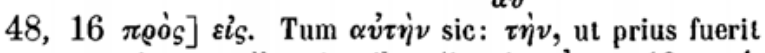
$\tau \dot{\eta} \nu$, superscripto paullo minoribus litteris $\alpha \dot{v}, 18 x \alpha \tau \dot{\varepsilon}-$

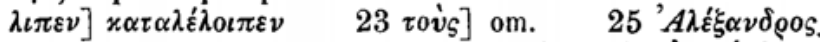
et seqq. rubro coI. superscriptum $+\beta \alpha \sigma \iota \lambda \varepsilon i \alpha \alpha \dot{\alpha} \lambda \varepsilon \dot{\xi} \alpha \dot{v} \delta \rho o v$

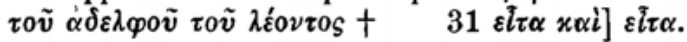

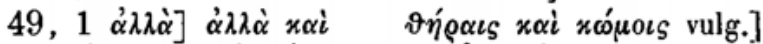

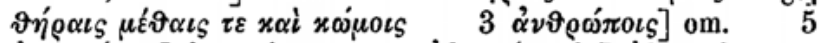

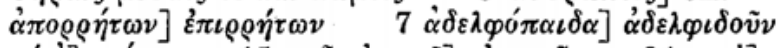

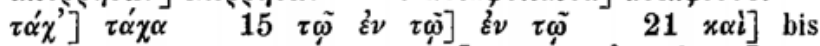
in fine et initio versus $25 \tau 0 \tilde{v}]$ addit $\left.{ }^{\prime} \varrho \omega \tau \tilde{\omega} \nu \tau o s\right] \mathrm{om}$.

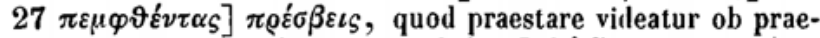

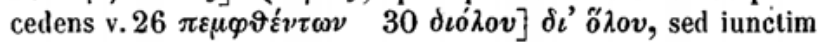
p. 53,8 .

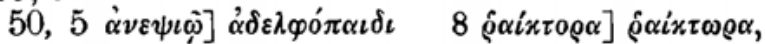
quod recepi, ut infra ex A 12 rubro col. superscr.: $\beta \alpha-$ $\sigma \iota \lambda \varepsilon l o v$ (sic, litteris $o v$ distincte scriptis, perspicue) $K \omega v \sigma \tau \alpha \nu$ -

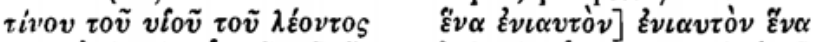

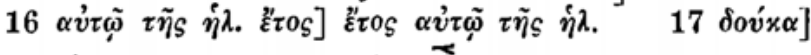

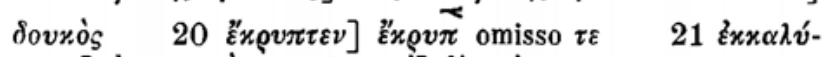

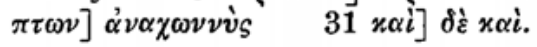

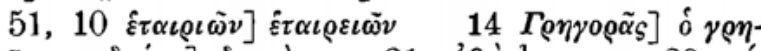

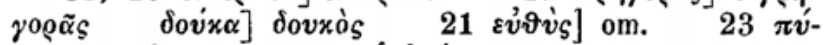

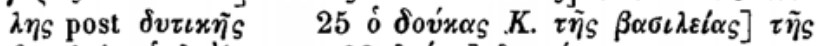

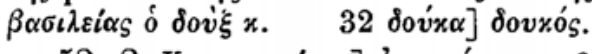

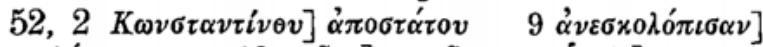

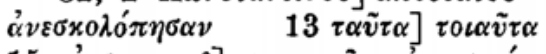

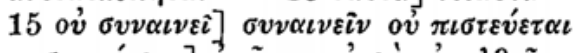

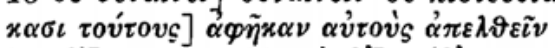
$21 \delta \dot{\varepsilon}]$ om. $\left.22 \tau \dot{\alpha} \delta^{\prime}\right] \tau \alpha^{\prime} \delta^{\prime}$ recte $\left.26 x \rho \alpha \tau \tilde{\eta} \sigma \alpha \iota\right]$ $\varkappa \rho \alpha \tau \eta^{\prime}-$ i. e. $x \rho \alpha \tau \dot{\eta} \sigma \alpha_{S}$, deinde accentus super $\tilde{\eta}$ emendatus et $\alpha \iota$ factum ex compendio syllabae $\alpha \mathrm{S} \quad 29 \delta \iota \varepsilon \nu \varepsilon \nu o ́ \eta \tau o]$

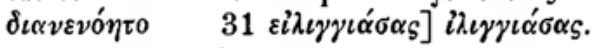

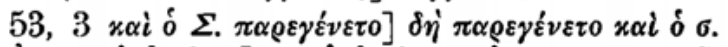

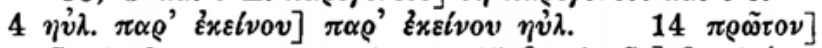
$\pi \varrho \tilde{\omega} \tau \alpha$ in fine versus et paginae $15 \beta \alpha \sigma \iota \lambda \varepsilon \iota \tilde{\omega} \nu] \beta \alpha \sigma \iota \lambda \varepsilon i \omega \nu$,

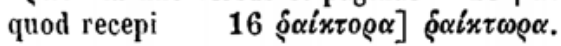


$54,11, i \delta \omega \nu] \dot{\delta} \varrho \tilde{\omega} \nu \quad 16 \dot{\alpha} \pi . \sigma v \mu \pi.] \sigma v \mu \pi . \dot{\alpha} \pi$.

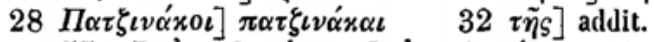

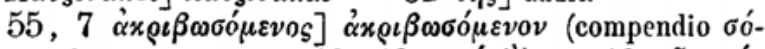

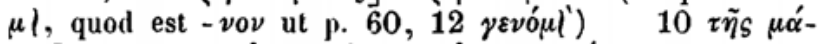

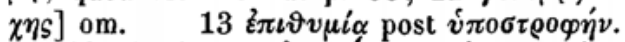

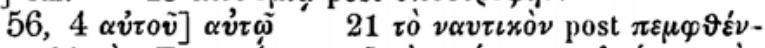

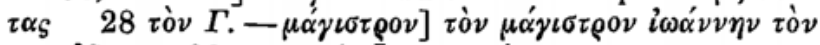

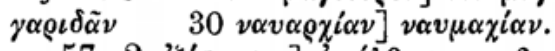

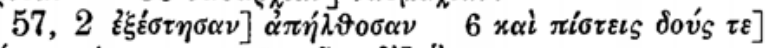

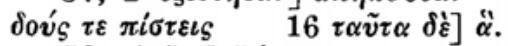

$\left.58,4{ }^{\prime \prime} \nu \alpha\right]$ in $^{\prime}$, quod recepi 13 rubro col. super-

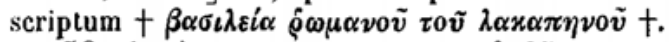

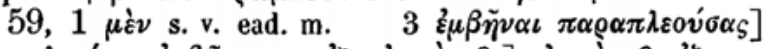

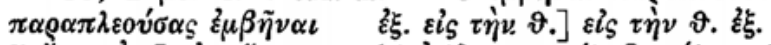

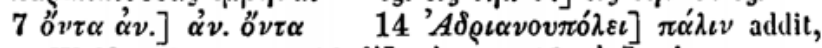

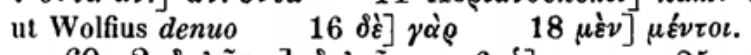

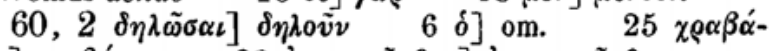

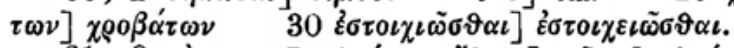

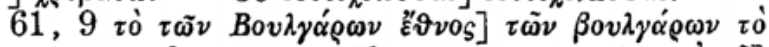

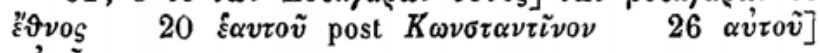
$\alpha \dot{v} \tau \omega$.

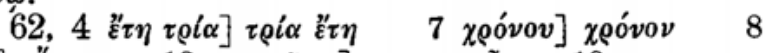

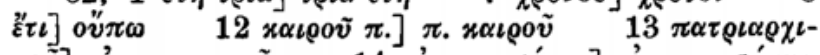

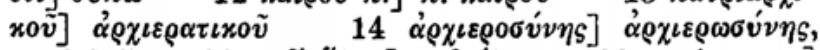
quod dedi $22 \mu \eta \delta \bar{\varepsilon}$ ö $\lambda \omega s] \mu \eta \delta \varepsilon{ }^{\prime} \lambda \omega s, 23 \pi \varepsilon \rho[\sigma \tau \alpha \nu \tau \alpha L]$

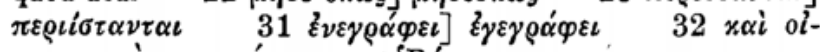

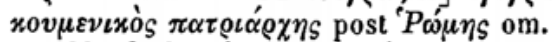

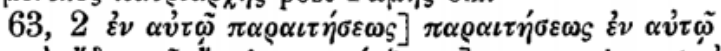

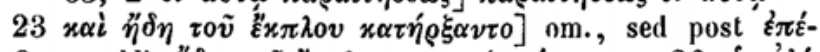

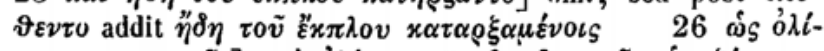

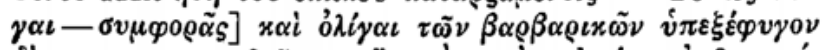

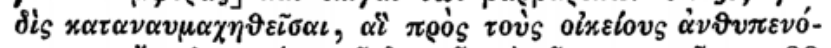

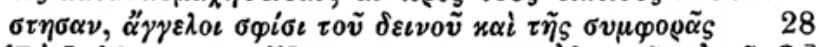

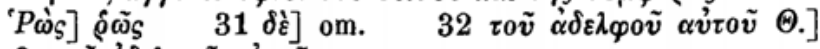

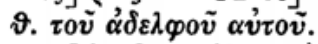

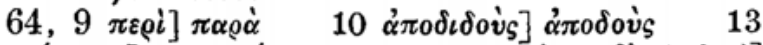

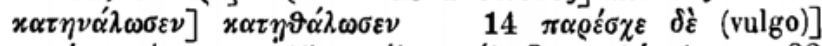

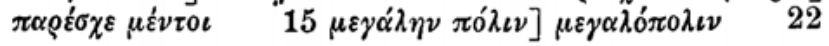




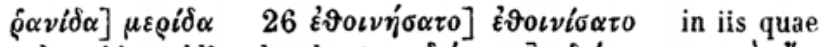

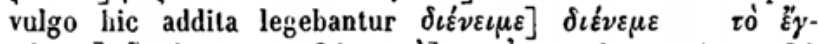
$x \lambda \eta \mu \alpha]$ है $\gamma x \lambda \eta \mu \alpha \quad 26 x \alpha \tau \grave{\alpha}]$ x $\alpha \tau$, quod recepi 28

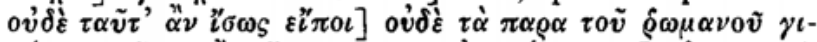

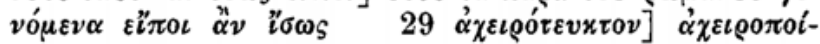
$\eta \tau$ זข

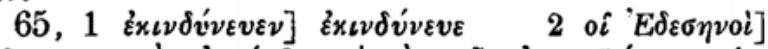

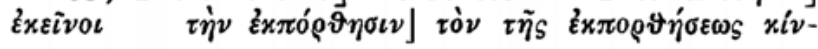

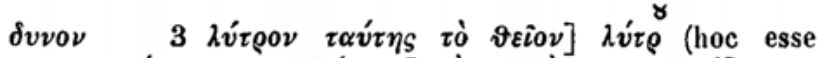

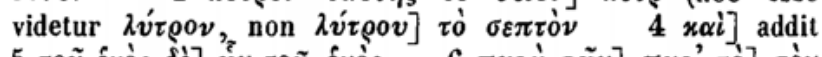

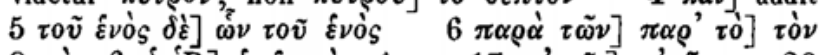

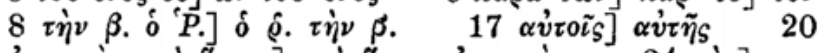

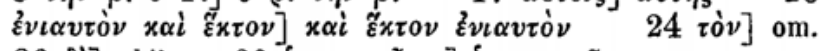
$26 \delta \dot{\varepsilon}]$ addit $30 \delta \mu \circ \varphi \omega \nu \tilde{\eta} \sigma \alpha \iota]$ o $\mu 0 \varphi \varrho 0 \nu \tilde{\eta} \sigma \alpha t$.

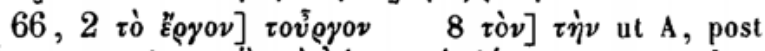

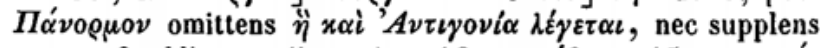
quae v. 9 addita pro iis ex A $16 \mu \varepsilon \tau \varepsilon \tau \varepsilon \hat{v} \eta$ v. 15 post $\tau \alpha v^{\prime}$ -

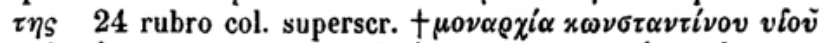

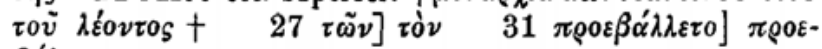
$\beta \dot{\alpha} \lambda \varepsilon \tau 0$.

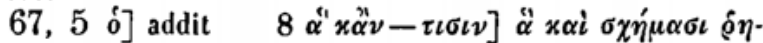

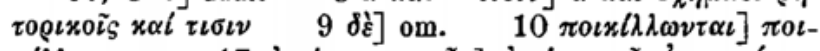

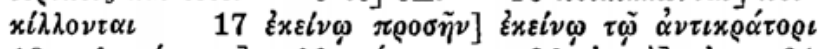

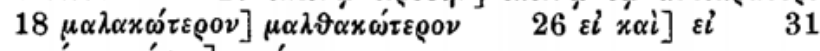

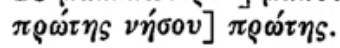

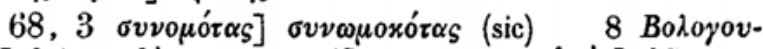

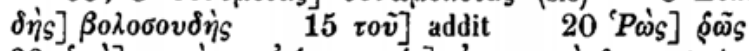

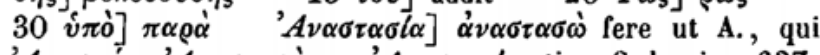

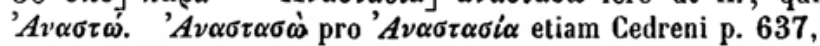
A, codex Coisl., quod ex $\Theta \varepsilon \circ \varphi \alpha \nu \omega$ natum videri potest, sed tribus libris tam sibi dissimilibus mirum si casu sit illatum.

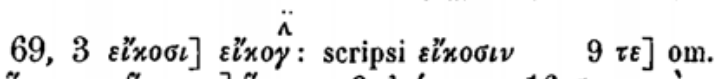

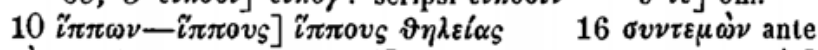

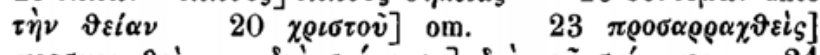

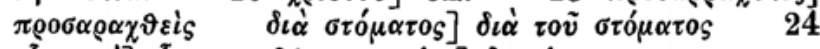

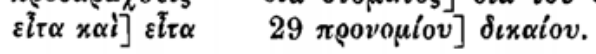




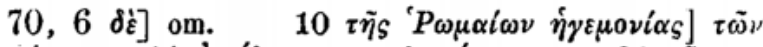

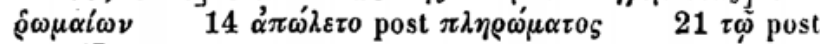
$\Phi \omega x \dot{\alpha}]$ om.

$\left.71,6 \alpha \dot{\tau} \tau \dot{\nu} \nu \delta v \sigma \chi \xi \rho \tilde{\omega}_{S}\right] \delta v \sigma \chi \varepsilon \rho \tilde{\omega}_{S} \alpha \dot{v} \tau \dot{\nu} \nu 12 \xi \xi \xi \omega-$

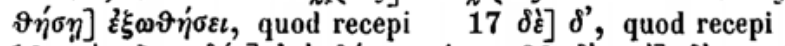

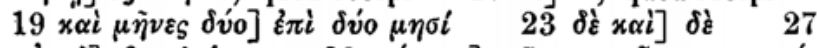
$\alpha \dot{v} \tau 0 \dot{v}] \beta \alpha \sigma \iota \lambda \varepsilon i \omega \nu \quad 29 \nu v x \tau \alpha \varsigma] \tau \tilde{\omega} \nu \nu v x \tau \tilde{\omega} \nu \quad \pi \alpha \rho \alpha^{\prime}-$ $\tau \iota \nu \omega \nu] \pi \alpha \varrho, \quad \lambda \iota \vartheta.] \lambda \iota \vartheta$. $\tau \iota \nu \omega \nu \nu$.

$72,1 \alpha \nu \vartheta \varrho \omega ́ \pi \omega \nu]$ om. 3 rubro col. superscr. $+\beta \alpha-$

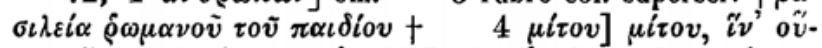

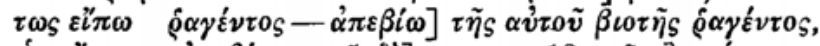

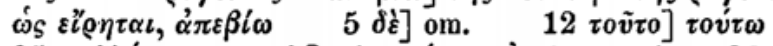
$\left.25 \pi 0 \lambda \lambda \alpha^{\prime} \iota_{S}-\sigma v \mu \pi \lambda.\right] \pi \lambda \varepsilon เ \sigma \tau \alpha^{\prime} \iota_{S} \alpha v \tau_{0} \tilde{i}_{S} \sigma v \mu \pi \lambda$. $26 \alpha i$ -

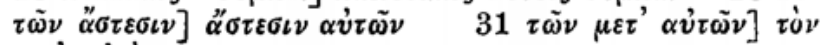
$\mu \varepsilon \tau^{\prime} \alpha \dot{v} \tau \dot{v} \nu$.

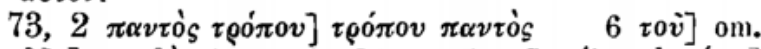

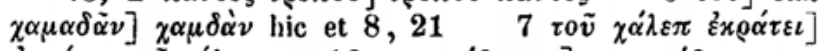

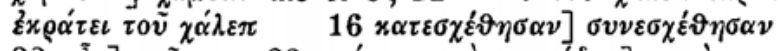

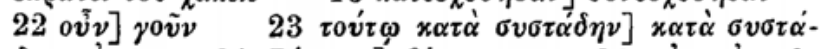

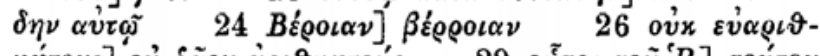

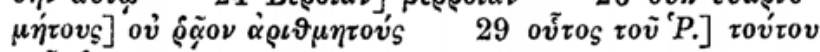
เoṽ

$\left.74,14 \alpha v \tau_{0} \hat{i}_{S} \delta \eta \lambda \alpha \delta \dot{\eta} \sigma v \gamma x o ́ \psi \alpha_{S}\right] \quad \delta \eta \lambda \alpha \delta \dot{\eta}_{i} \sigma v \gamma x o ́ \psi \alpha_{S}$

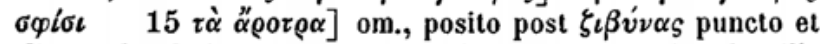
aliquot deinde litterarum spatio relicto, quod capiat duo illa

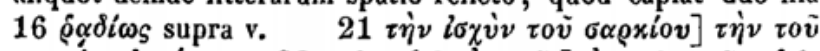

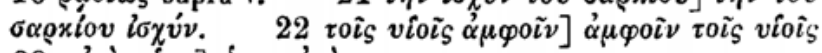
$28 \alpha \dot{v} \tau \dot{\nu} \nu \dot{v} \varphi.] \dot{v} \varphi$. $\alpha \dot{v} \tau \dot{v} \nu$.

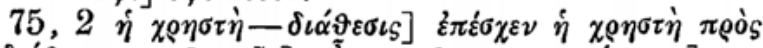

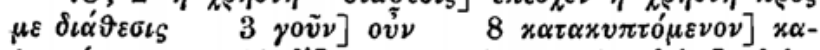

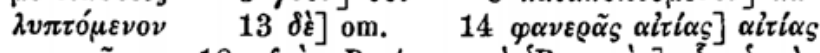

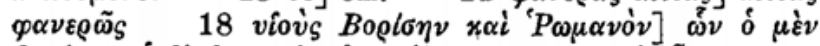

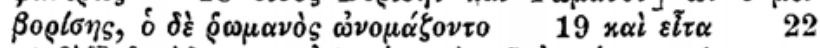

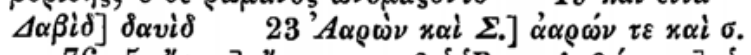

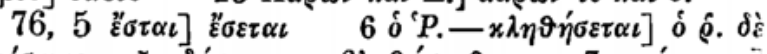

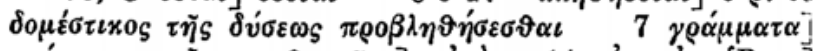

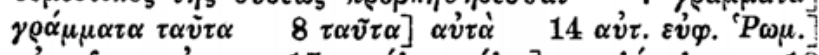

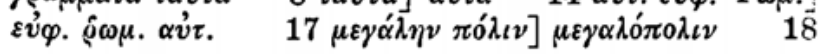




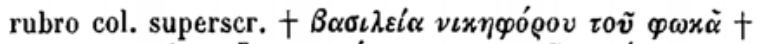

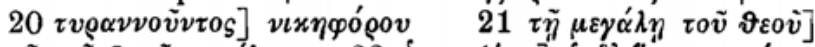

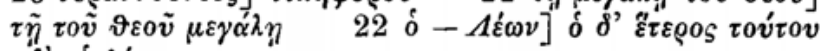
viòs ò $\lambda \varepsilon ́ \omega \nu$.

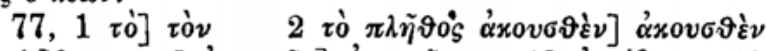

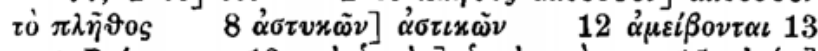

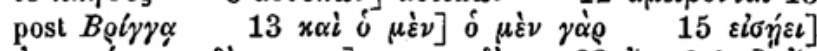

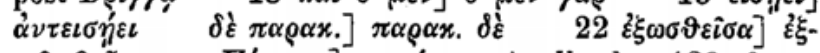

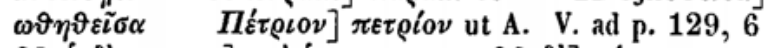

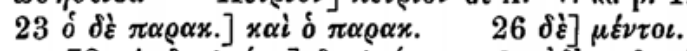

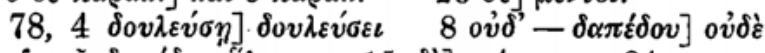

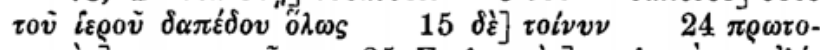

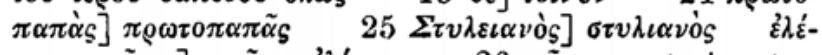

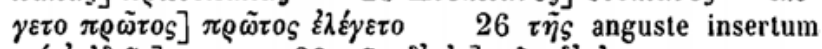
$\left.\mu \eta^{\prime} \tau i \delta \varepsilon \tilde{\imath} \nu\right]$ om. $\left.29 \tau \tilde{\omega} \nu \delta^{\prime} \dot{\varepsilon} \nu\right] \tau \tilde{\omega} \nu \delta^{\prime}, \varepsilon$.

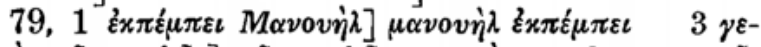

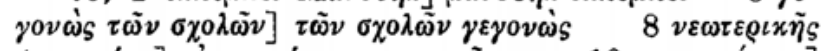

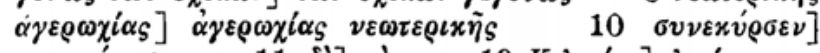

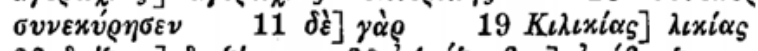

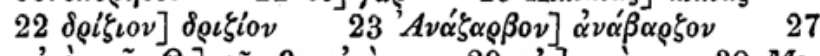

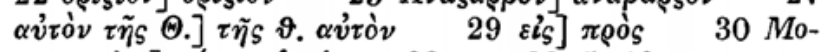

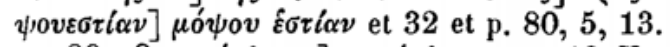

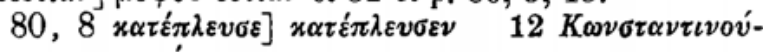
$\tau v^{\prime}$

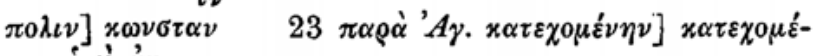
$v \eta v i j \dot{\alpha} \alpha \gamma$.

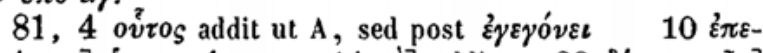
$\chi \omega \rho i \alpha \sigma \varepsilon v]$ v $\pi \varepsilon \chi \omega \varrho\left[\alpha \sigma \varepsilon v \quad 14 \tau{ }^{\circ}\right]$ addit $\left.28 \delta \varepsilon \delta \nu \tau \alpha \tau \tilde{\omega} \nu\right]$ $\tau$ :

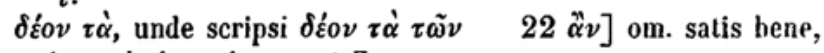
etsi saepissime sic peccat Zonaras.

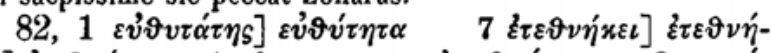

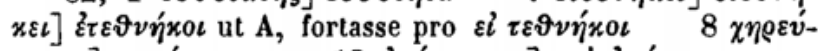

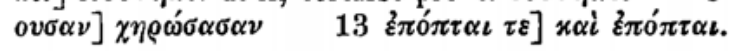

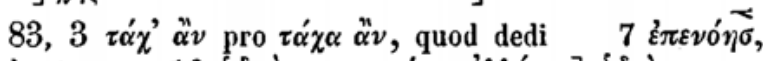

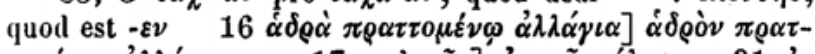

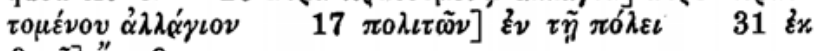

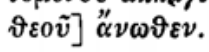


84, $7 \pi \varepsilon \varrho \iota \pi . \tau \dot{\eta} \nu \varphi . \dot{\alpha} \sigma \varphi \alpha \lambda \dot{\eta}] \dot{\alpha} \sigma \varphi \alpha \lambda \tilde{\eta} \pi \varepsilon \rho \iota \pi . \varphi . \quad 9$

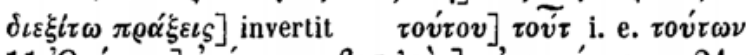

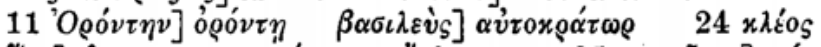

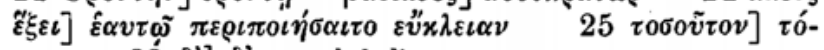
GOv $32 \delta \dot{\varepsilon}] \delta$, quod dedi.

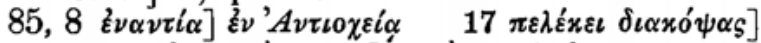

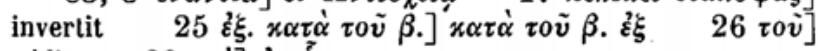
addit $29 x \alpha i] \dot{\varepsilon} \nu \stackrel{?}{\eta}$.

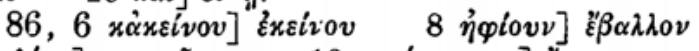

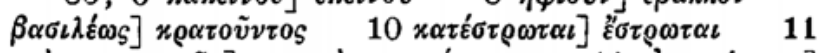

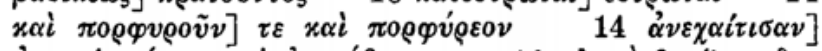

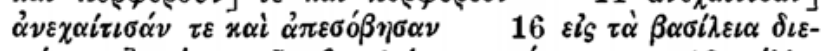

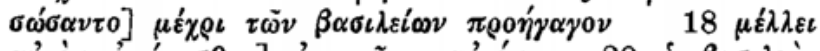

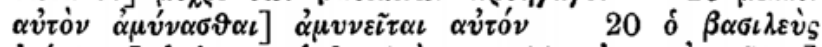

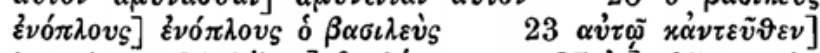

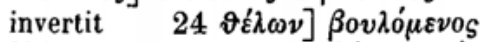

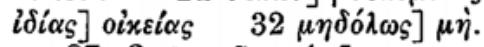

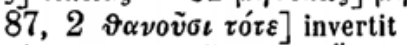

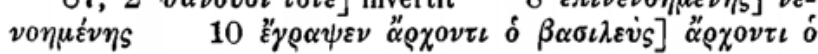
$\beta \alpha \sigma \iota \lambda \varepsilon \dot{v} \varsigma$ है $\gamma \varrho \alpha \psi \varepsilon \quad 25 \alpha \pi \dot{\eta} \lambda \alpha \sigma$ i. e. $\sigma \varepsilon \nu$ potius quam $\sigma \varepsilon$.

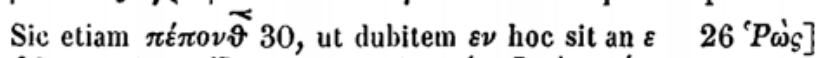

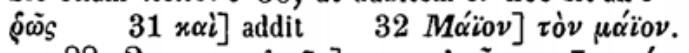

88, $2 \tau \alpha \mu \varepsilon\llcorner 0 v \lambda x \tilde{\omega} \nu] \tau \iota \mu \iota 0 v \lambda x \tilde{\omega} \nu \quad 7 \pi \rho \omega ́ \eta \nu$ s. v. ead. m. $\left.\mu \varepsilon \gamma \alpha \lambda_{0} \rho \varrho \varepsilon \varepsilon \varepsilon \iota \alpha \nu\right]$ om. 13 o门] $\delta$ perspicue 14 है $\varphi \alpha-$

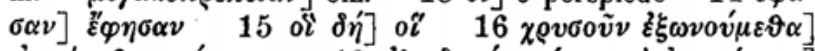

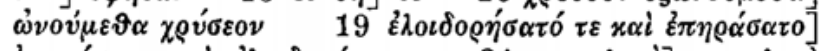

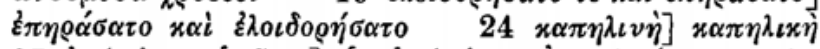

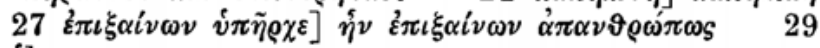
o] om.

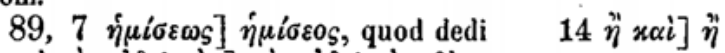

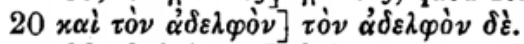

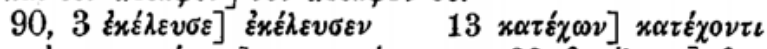

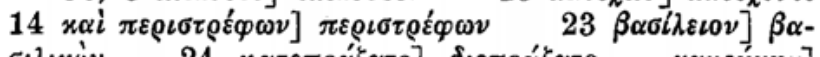

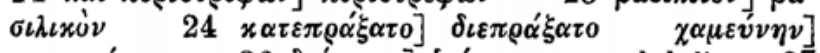

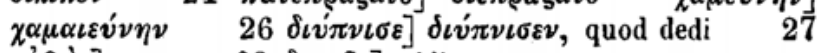
$\varepsilon \dot{v} \hat{v} \varsigma$ ] om. $\left.28 \delta \varepsilon \iota \nu \tilde{\omega}_{\varsigma}\right]$ addit.

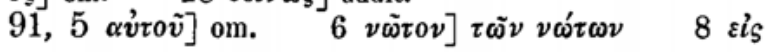




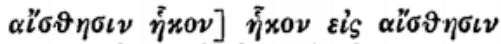

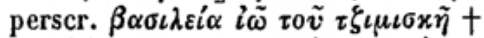

$\left.91,10 \tau 0 \tilde{\iota}_{S} \sigma . \delta \iota \dot{\alpha} \vartheta.\right] \delta \iota \dot{\alpha} \vartheta$. $\tau 0 \tilde{i}_{S} \sigma$.

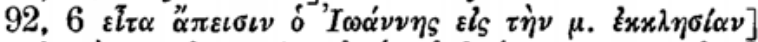

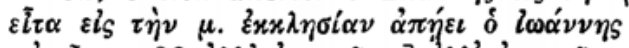

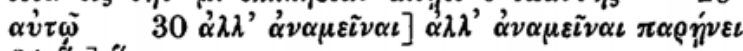
31 ố 5$]$ ö $\sigma \pi \varepsilon Q$.

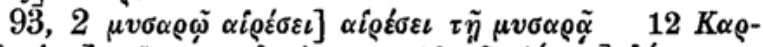

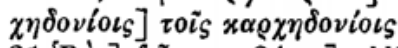

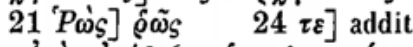

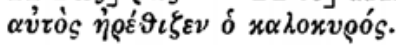

21 rubro col. su$26 \tau \dot{\eta} \nu]$ om.

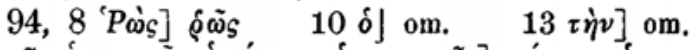

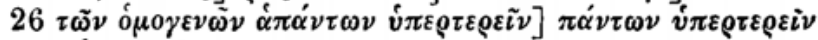
$\tau \tilde{\omega} \nu \dot{\delta} \mu о \gamma \varepsilon \nu \tilde{\omega} \nu$.

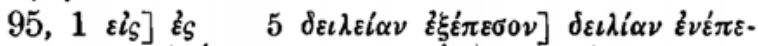

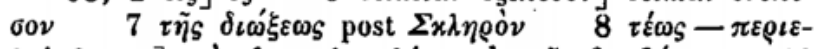
$\lambda \varepsilon i \varphi \vartheta \eta \sigma \alpha \nu] x \alpha i$ oi $\pi \varepsilon \rho \iota \lambda \varepsilon \iota \varphi \vartheta \varepsilon \dot{\varepsilon} \nu \varepsilon s$ हो $\tau \omega \check{\nu} \beta \alpha \rho \beta \alpha \rho \omega \nu 10$

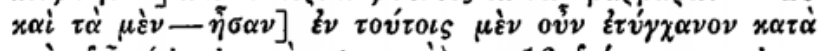

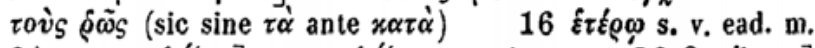
$\left.24 \pi \alpha \varrho \alpha \varphi v \lambda \alpha^{\prime} \xi \alpha \iota\right] \pi \varepsilon \rho \iota \varphi v \lambda \alpha^{\prime} \xi \iota \iota$ perspicue $\left.26 \beta \alpha \sigma i \lambda \varepsilon \iota \sigma_{s}\right]$ ह่x $\beta \alpha \sigma \iota \lambda \dot{\varepsilon} \omega s$.

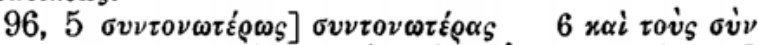

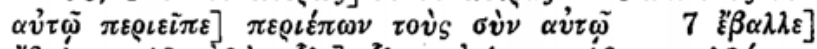

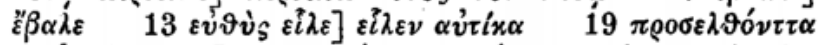

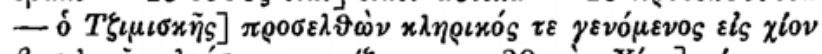

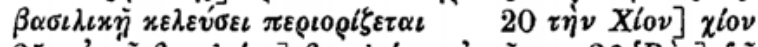

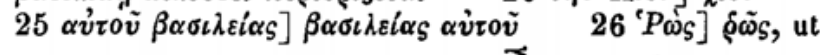
97,$8 ; 98,4$ et semper $\quad 30 \delta \iota \dot{\varepsilon} \lambda \alpha \vartheta$ ante $\pi \rho o$ s. Ergo $\varepsilon \nu$ potius quam $\varepsilon$.

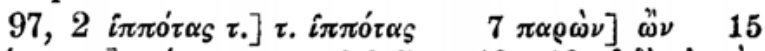

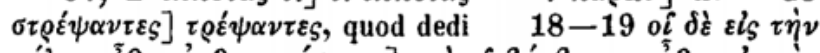

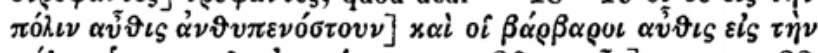

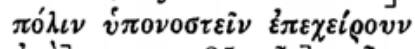

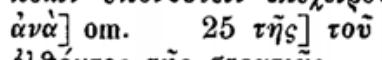

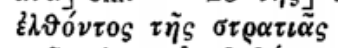

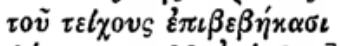

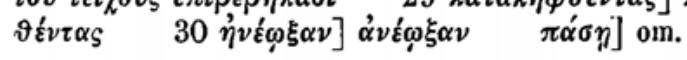

$20 \tau \iota \nu \tilde{\omega} \nu]$ om. 22

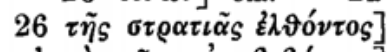

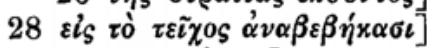
$\left.29 x \alpha \tau \alpha \lambda \eta \varphi \vartheta \dot{\varepsilon} v \tau \alpha_{\varsigma}\right] x \alpha \tau \alpha \lambda \varepsilon \iota \varphi-$ 


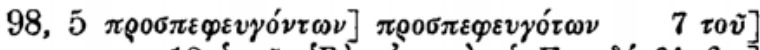

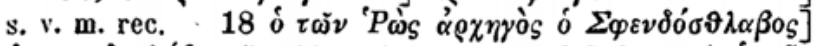

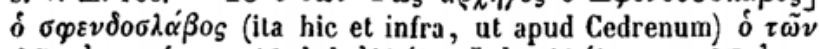

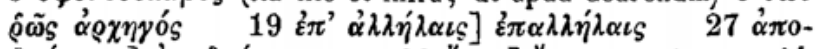

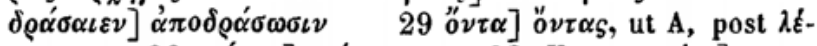
$\gamma \varepsilon \tau \alpha \iota$, et $\left.\left.30 \pi \alpha^{\prime} \nu \tau \alpha\right] \pi \alpha^{\alpha} \nu \tau \alpha_{S} \quad 32 K \omega \nu \sigma \tau \alpha \nu \tau i \alpha_{S}\right] x \omega \nu$ -

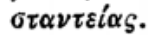

$\left.99,16 x \alpha \tau^{\prime} \alpha_{\alpha}^{\prime \prime} \lambda \alpha_{S} x \alpha i \stackrel{\alpha}{\alpha} \lambda \lambda_{S}\right] x \alpha \tau \alpha \dot{\alpha} \tau \iota \nu \alpha_{S} x \alpha i \stackrel{\alpha}{\alpha} \lambda \lambda_{\alpha_{S}}$ $17 x \alpha i$ $\left.\lambda \alpha \vartheta{ }^{\prime} \nu \tau \varepsilon \zeta-\dot{\varepsilon} \pi \alpha \nu \eta^{\prime} \lambda \vartheta 0 \sigma \alpha \nu\right]$ om. $\left.27 \delta \dot{\varepsilon} x \alpha i\right] \delta \dot{\varepsilon}$, omisso etiam $\delta \dot{\varepsilon}$ post $\left.\lambda_{\iota \mu \mu \tilde{\omega}} 28 \tau \tilde{\eta} \zeta\right]$ om. 29 ov $\mu \varepsilon^{\prime} \nu$ -

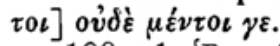

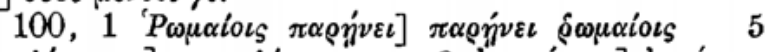

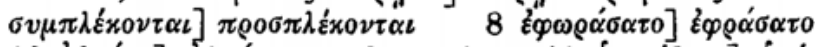

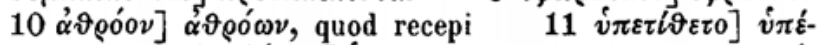

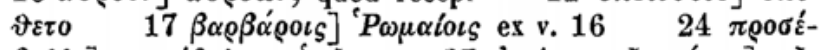

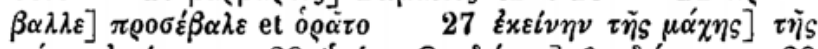

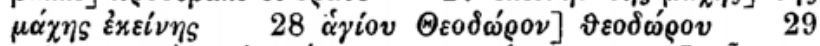

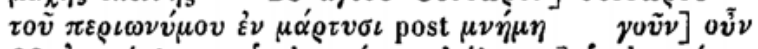

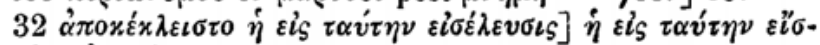

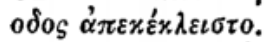

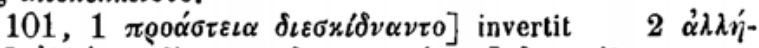

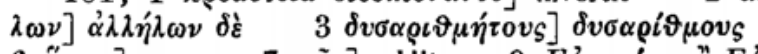

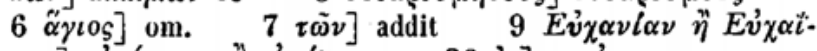

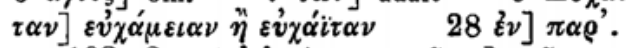

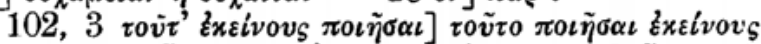

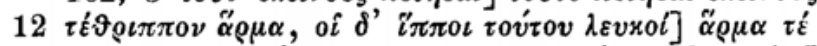

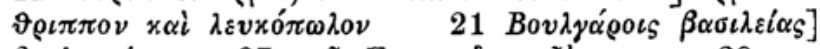

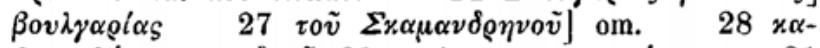

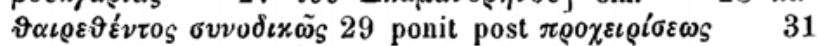

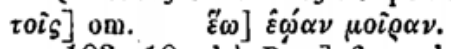

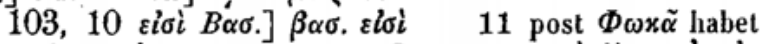

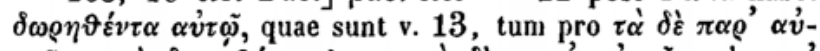

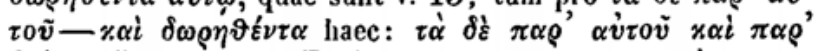

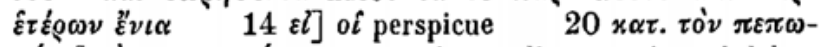

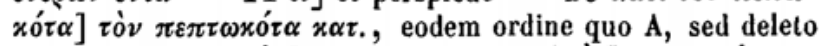
deinde $\tau \quad 23 \tau \dot{\eta} \nu]$ om. $26 \mu \varepsilon \tau \alpha \sigma \tau \dot{\alpha} \varsigma] \mu \varepsilon \vartheta \iota \sigma \tau \alpha \dot{\alpha} \mu \varepsilon \nu \sigma_{S}$

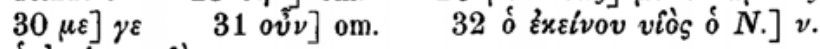
ó éxeívov viós. 
$104,8 \sigma v \nu 0 \mu 0 \tau \omega \nu] \sigma v \nu \omega \mu 0 \tau \tilde{\omega} \nu$ iò $\mu . x \alpha \tau \alpha \gamma \gamma \varepsilon i \lambda \alpha \nu \tau 0_{S}$

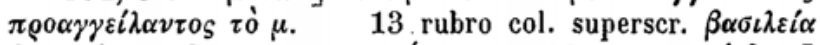

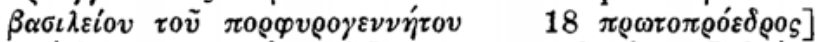

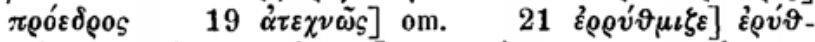
$\mu \iota \zeta \varepsilon \quad 22 \mu \varepsilon \tau \alpha \chi \varepsilon i \varrho \eta \sigma \iota \nu], \mu \varepsilon \tau \alpha \chi \varepsilon i \varrho \iota \sigma \iota \nu \quad 28 \varepsilon \sigma \alpha 0 \lambda \alpha-$

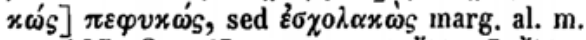

$105,2 \tau 0 \tilde{v}]$ addit $4 \not \alpha \lambda \lambda \omega \varsigma] \alpha^{\prime} \lambda \lambda \omega$ perspicue 11

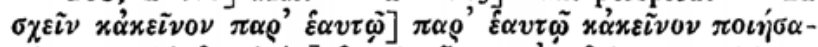

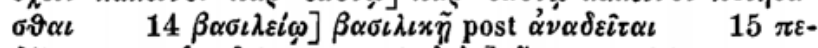

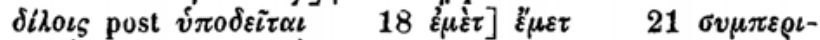

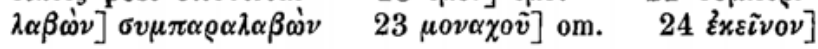

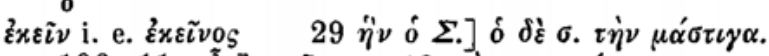

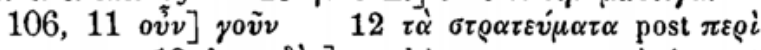
$\left.\Lambda i \pi \alpha \varrho \alpha v \quad 13 \lambda_{\iota x \alpha \nu \delta o ̀}\right] v$ ultimum $\mathrm{m}$. recentissima et pallido atramento in $\sigma$ mutatum, ut Wolfius in interpr. lat.

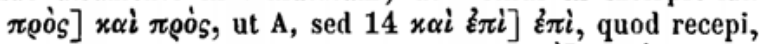
etsi alterum non notatum ex A $23 \pi \alpha \rho \dot{\alpha}] \quad \pi \varepsilon \rho i \quad 26$

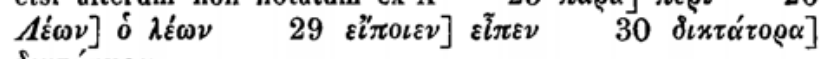
$\delta\llcorner\kappa \tau \alpha \tau \tau \omega \rho \alpha$.

$\left.107,2 \tau^{\prime}\right] \tau$ cum lacuna tertiae partis versus. Omittit illud $\tau^{\prime} \mathrm{A}$, ut Wolfius. Ducangius vero haud dubie ex alio addidit libro $8 \pi \rho \omega \tau o \beta \varepsilon \sigma \tau \iota \alpha \rho i ́ o v] \tilde{\alpha} \beta \varepsilon \sigma \tau \iota \alpha \varrho i o v$ hic, non $\left.12,1416 \eta^{\prime} \nu \varepsilon \gamma x \varepsilon\right] \eta \nu$ initio versus, supra quem prominet, in ras., fortasse etiam $\varepsilon \gamma$. Potuit pr. esse $\dot{\varepsilon} v \tilde{\eta} x \varepsilon$, quod alibi in hac formula cum

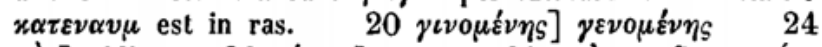

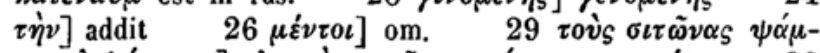

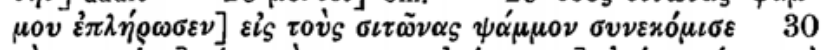

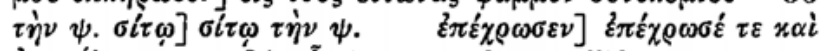

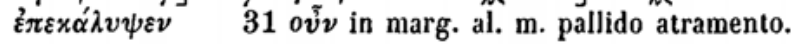

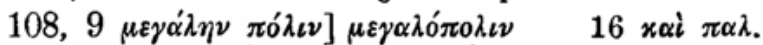

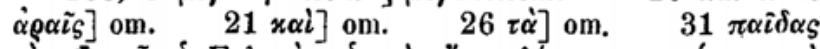

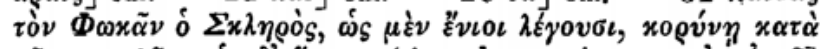

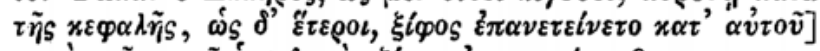

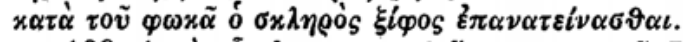

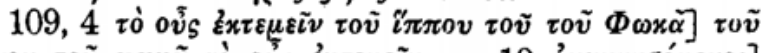

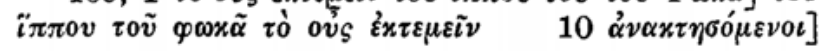




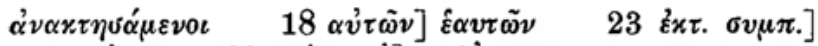

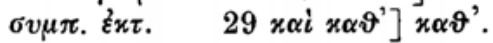

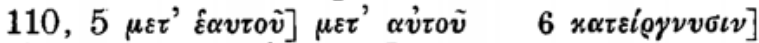

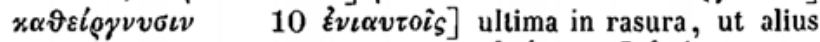

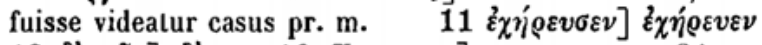

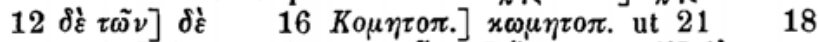

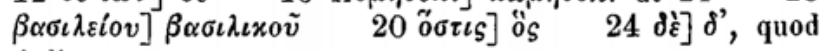
dedi.

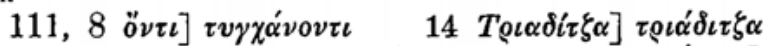

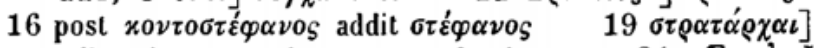
$\alpha$ medium in ras., sed ut pr. non fuerit $\tau \alpha \quad 21$ oi $], \alpha v \tau \omega$

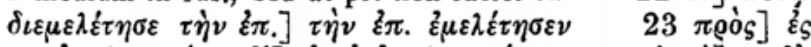
$\left.25 \dot{\alpha} \nu \alpha \zeta \varepsilon v \gamma v v_{\varepsilon} \varepsilon v \delta \dot{\varepsilon}\right] \dot{\alpha} \lambda \lambda^{\prime} \dot{\alpha} v \alpha \zeta \varepsilon v \gamma v v_{\varepsilon}\left(v \quad x \alpha i \mu \dot{\eta}^{\prime}\right] \mu \eta \delta^{\prime}$.

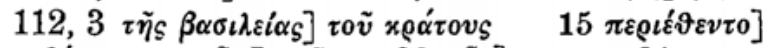
$\pi \varepsilon \rho \iota \tau \iota \vartheta \varepsilon \dot{\varepsilon} \alpha \iota$ ante $\tau \tilde{\omega} \Phi \omega x \tilde{\alpha}, 20 \tau \tilde{\omega} \nu]$ om. $21 \pi \alpha \rho \alpha-$

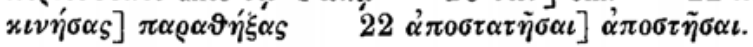

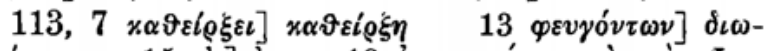

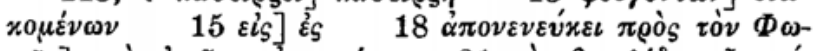

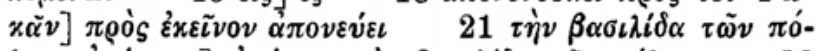

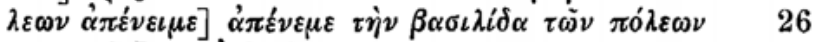
$\gamma \alpha \vartheta \iota \varepsilon \rho \tilde{\omega} \nu] \psi \alpha \vartheta^{\prime} i \varepsilon \rho \tilde{\omega} \nu$, quod recepi.

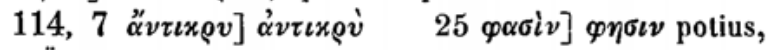
i.

sic: $\varphi \eta$

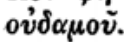

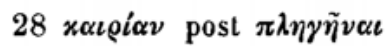

$32 \mu \eta \delta \alpha \mu \eta \overline{]}]$

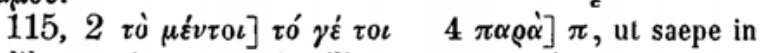
hoc libro, scriptum non intelligens corrector in marg. $m$. rec.

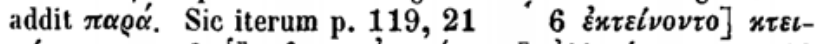

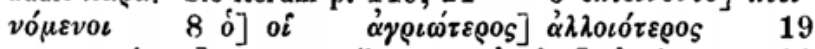

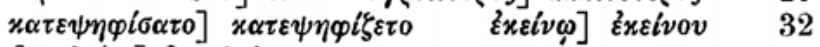
$\left.\beta \alpha \sigma \iota \lambda \varepsilon i \alpha_{\S}\right] \beta \alpha \sigma \iota \lambda \varepsilon i o \iota s$.

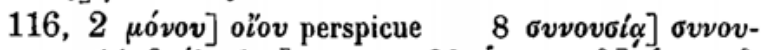

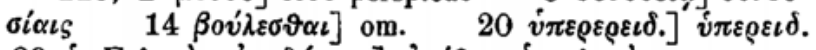

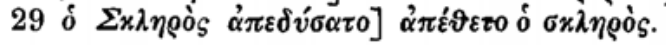

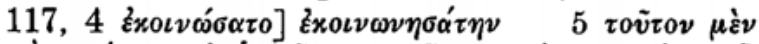

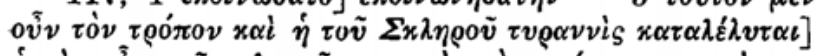

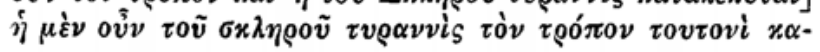




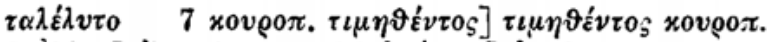

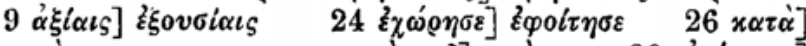

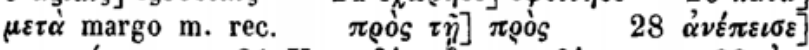

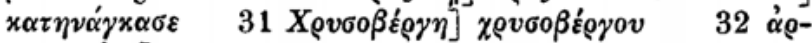
$\chi\llcorner\varepsilon$ Q $\sigma u ́ v \eta]$ per $\omega$.

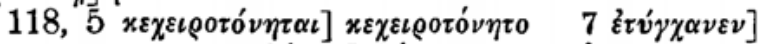

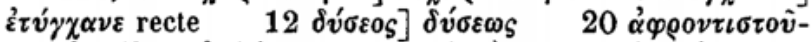

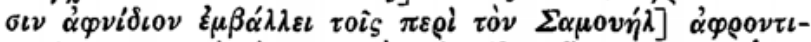

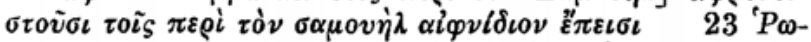

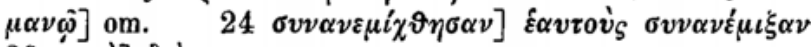
$30 \pi \alpha \rho \dot{\alpha}] \delta \iota \dot{\alpha}$.

$119,3 \tau \dot{0} v]$ om. $\quad 4 B \iota \delta i v \eta s] \beta v \delta i v \eta s$ et $9 \beta v \delta i v \eta v$, haud dubie pro $\beta \iota \delta v i v \eta_{s}$, quod est apud Cedrenum p. 705, C,

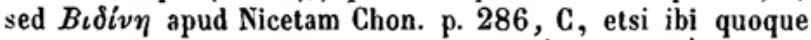

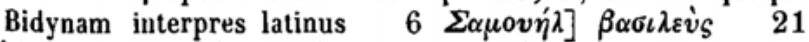

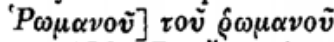

$22 \tau 0 \tilde{v} v[0 \tilde{v}]$ vio v.

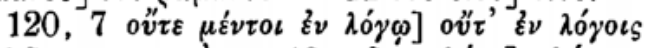
11

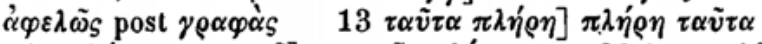
$\left.14 \tau \alpha \lambda \alpha^{\prime} \nu \tau \omega \nu \chi \varrho v \sigma o \tilde{v}\right] \chi \varrho v \sigma o \tilde{v} \tau \alpha \lambda \alpha^{\prime} \nu \tau \omega \nu, 20$ inter $x \lambda \tilde{\eta} \sigma \iota \varsigma$ et oi trium fere litterarum spatium 22 ' $\nu \varepsilon x o ́ \sigma \mu o v \nu]$ ह́xó-

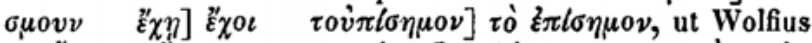

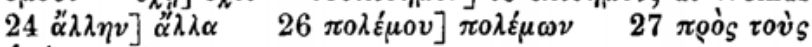
vं $\pi \dot{o} \chi \varepsilon \hat{\imath} \rho \alpha]$ om.

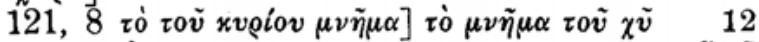
$\alpha \pi 0 x \lambda \varepsilon \tilde{\sigma} \sigma \alpha l] \dot{\alpha} v 0 x \lambda \varepsilon \tilde{\varepsilon} \sigma \alpha t, \mathrm{~m}$. rec. $v$ in $\pi$ mutato $\left.19 \varepsilon^{\prime \prime} v \alpha\right]$

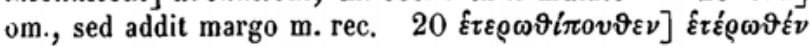
$\pi 0 \vartheta \varepsilon v$.

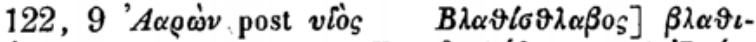

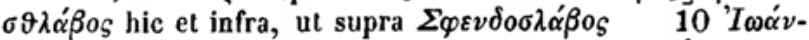
$v \eta \varsigma]$ ] $\alpha i$ i $i \omega \alpha v \nu \eta s:$ conf. p. 123, 2, $1023 \alpha v \tau \omega]$ om.

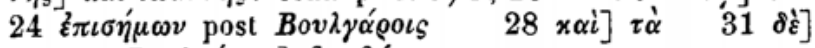
on. $\quad B v v \lambda \gamma \alpha \rho \omega \nu] \beta \alpha \rho \beta \alpha ́ \rho \omega \nu$.

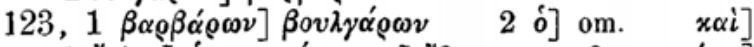

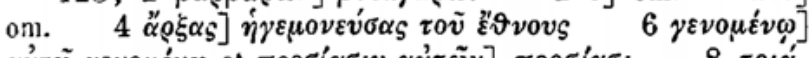
$\alpha \dot{v} \tau \tilde{\omega} \gamma \varepsilon v 0 \mu \varepsilon^{\prime} v \omega$ et $\left.\pi \rho 0 \sigma i \alpha \sigma \iota \nu \alpha \dot{v} \tau \tilde{\omega} \nu\right] \pi \varrho \circ \sigma i \alpha \sigma \iota \quad 8 \tau \rho \iota \alpha^{\prime}-$

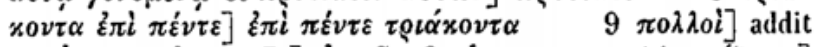

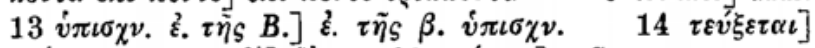

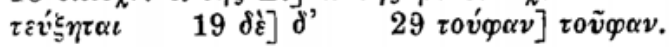




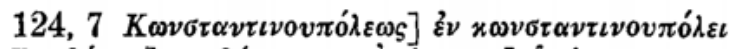

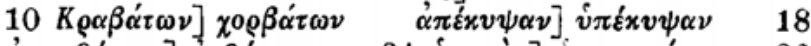

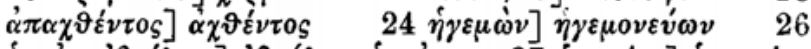

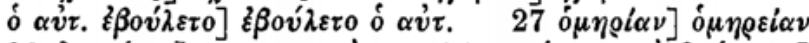

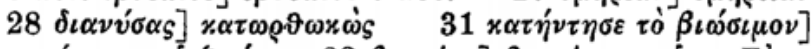

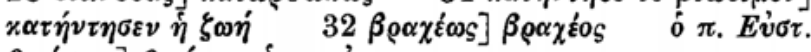
$\left.\vartheta \nu \eta^{\prime} \sigma x \varepsilon l\right] \vartheta \nu \eta^{\prime} \sigma \varepsilon \iota$ o $\pi . \varepsilon \dot{v} \sigma \tau$.

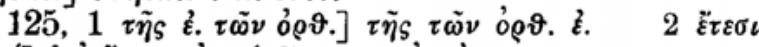

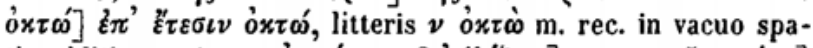

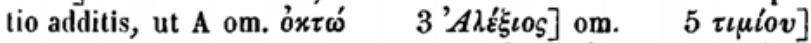

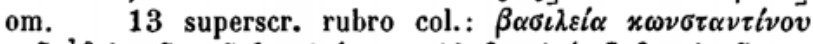

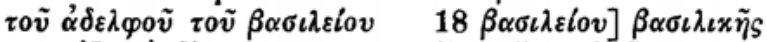

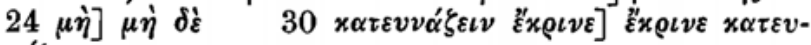
$\nu \alpha \dot{\xi} \varepsilon \varepsilon v$.

$126,15 \lambda 0 \iota x \mu \omega \tilde{\omega}] \lambda 0 \iota \mu \iota x \omega \tilde{\omega} \quad 16 x \alpha \vartheta$

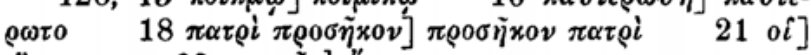
oi recte $32 x \rho \alpha \tau \omega \nu]$ 이 $\varrho \nu \nu$.

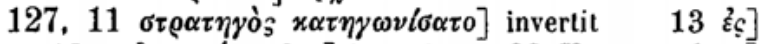

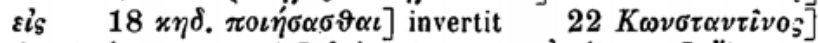

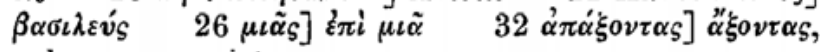
ead. m. superscripto $\pi \alpha$.

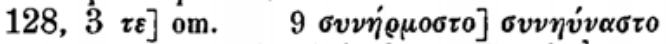
13 superscr. rubro col.: $\beta \alpha \sigma \iota \lambda \varepsilon i \alpha \hat{\varrho} \omega \mu \alpha v o \tilde{v}$ voṽ $\alpha$ Q $\gamma v \rho o \tilde{v}$

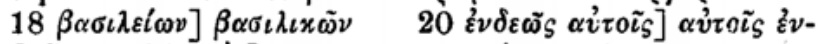

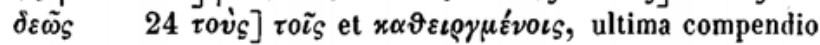
scripta.

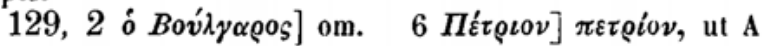
et Cedrenus p. 729, C, quod scripsi, ut supra p. 77, 228

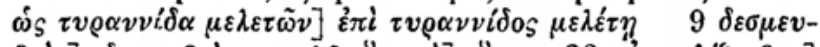

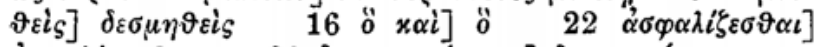

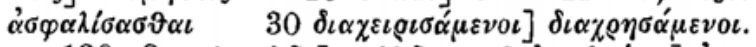

$130,8 \pi 0 \lambda v \tau \varepsilon \dot{\lambda} \tilde{\omega} \nu] \pi 0 \lambda \lambda \hat{\omega} \nu$ $9 \dot{\varepsilon} \sigma \tau \alpha \lambda \mu \varepsilon \dot{\varepsilon} \nu 0 l] \dot{\alpha} \pi \varepsilon \sigma \tau \alpha \lambda-$

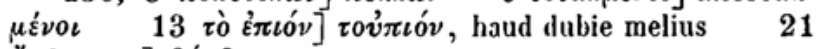

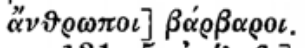

$131,5 \dot{\alpha} \nu \varepsilon \hat{\varepsilon} \alpha \beta \varepsilon] \dot{\alpha} \nu \varepsilon^{\prime} \beta \alpha \lambda \varepsilon$, quod recepi. Idem vilium in Paris. p. 129, B, et alibi notavi ad Stephanum v. $\alpha^{\prime} \nu \alpha \beta \dot{\alpha} \alpha \lambda \lambda \omega$

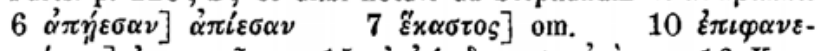

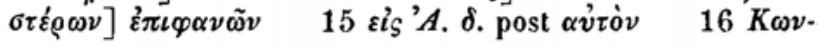




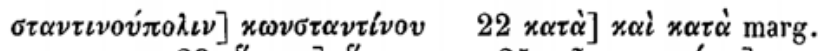

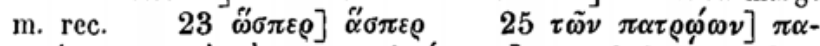

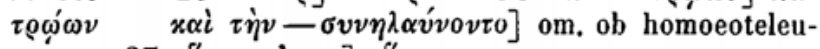

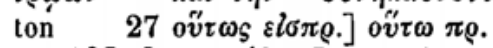

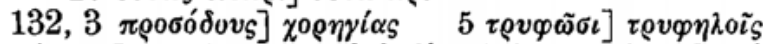

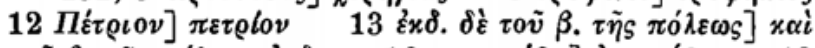

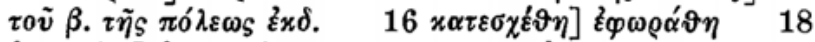

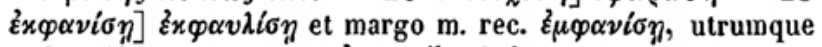
male. Nam rarissimum $\varepsilon_{x} x \alpha \nu l \xi \omega$ habet etiam Cedrenus p.

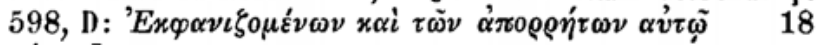

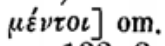

133, $3 \lambda \iota \mu o v i] \lambda \iota \mu o \dot{\nu}$, correctus m. r. $\left.\lambda \eta v \pi \delta^{\prime} \iota v\right] \tau \dot{\eta} v \mu \varepsilon \gamma \alpha \lambda o^{\prime} \pi 0 \lambda \iota v$ fere ut A $\left.\sigma \mu \alpha \sigma \iota \quad 29 \pi \alpha^{\prime} v \tau \alpha \mu \alpha \dot{\alpha} \tau \nu\right] \mu \alpha^{\prime} \tau \nu \nu \pi \alpha^{\prime} v \tau \alpha$ quod usitatius: sed $\pi \varepsilon \rho i$ supra p. 164, C.

$$
\begin{array}{r}
13 \tau \grave{\eta} \nu \mu \varepsilon \gamma^{\prime}- \\
26 \chi \rho i \mu \alpha \sigma i] \chi \rho^{i}- \\
31 \pi \varepsilon \rho i] \pi \rho o s,
\end{array}
$$

134, $8 x \alpha i]$ om.

$\left.9 \quad \varepsilon l_{S}\right]$ हैs

$11 \vartheta \alpha \lambda \alpha \mu \eta \pi 0 \lambda_{0} \tilde{\sigma} \sigma$

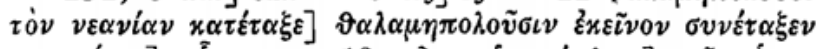

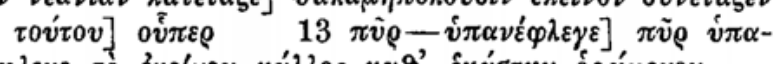

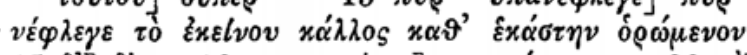

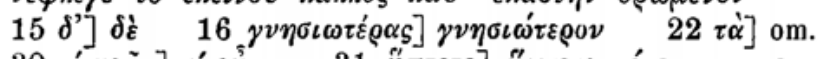

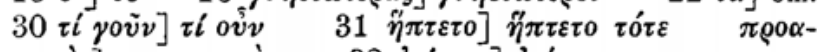

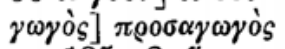

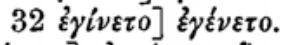

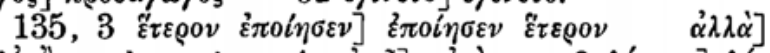

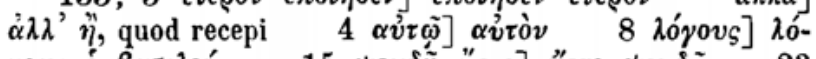
rovs of $\beta \alpha \sigma \iota \lambda \varepsilon v_{S}$ $\tilde{\alpha} \sigma \vartheta \mu \alpha] \alpha \sigma \vartheta \mu \alpha$

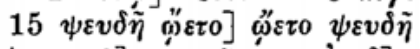

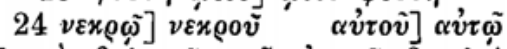

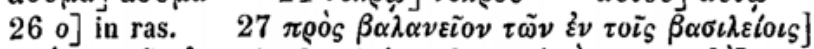

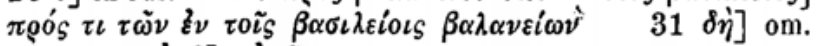
$32 \tau \iota v \alpha \mathcal{\alpha} \alpha \tau \tilde{v}] \alpha \hat{v} \tau \tilde{\omega}, \tau \iota v \alpha \varsigma$.

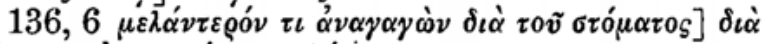

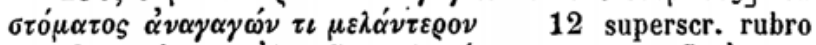

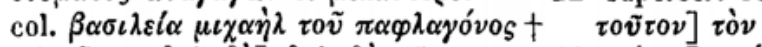

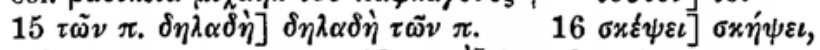
sed $\mathrm{m}$. rec. $\varepsilon$ super $\eta \quad 18 \pi \alpha \varrho \dot{\alpha}]$ in cod. scriptum ut saepius $\boldsymbol{\pi}^{\varepsilon}$ corrector in marg. correxit $\pi \alpha \rho \alpha$, idem non faciens

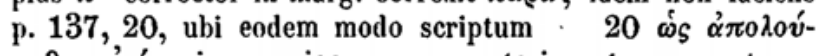
$\mu \varepsilon \vartheta \alpha \alpha v \tau i x \alpha$ in margine $\mathrm{m}$. rec., asterisco tamen post $\mu t$ - 


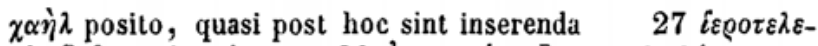

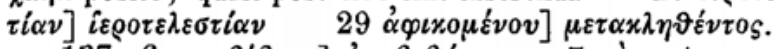

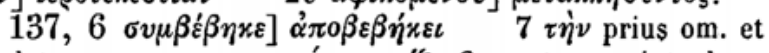
supplet margo m. rec. $\tau \alpha v^{\prime} \eta \eta \chi \alpha \varrho i \zeta \varepsilon \sigma \vartheta \alpha \iota$, ut supra interdum, integrum faciunt folii $503 \mathrm{r}$. versum postremum ab initio et

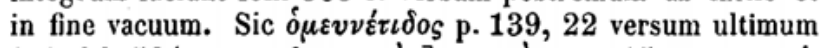
facit fol. $504 \mathrm{r}$. $9 \mu \alpha x \rho \dot{\alpha} v] \mu \alpha x \rho \dot{\nu} v \quad 15 \gamma v v \alpha \iota x \omega v i$ -

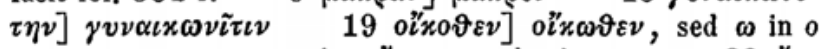
mutato m. rec., quae etiam है $\chi$ cov supplet in marg. $\quad 22 \not \prime \nu$ -

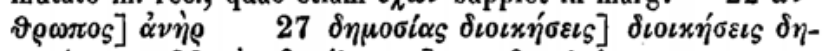
$\mu 0 \sigma i \alpha s \quad 28 \tau \dot{\eta} v \beta \alpha \sigma i \lambda \iota \sigma \sigma \alpha v] \tau \tilde{\eta} s \beta \alpha \sigma \iota \lambda \varepsilon i \alpha_{S}$, per compendium scriptum ut p. 138,3 , margo m. rec. corrigit $\tau \dot{\eta} v$ $\beta \alpha \sigma i \lambda \iota \sigma \sigma \alpha \nu$.

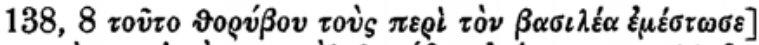

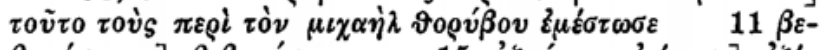

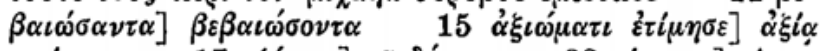

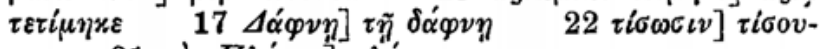

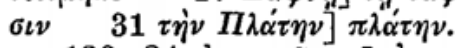

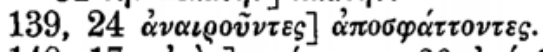

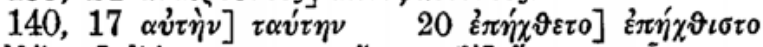

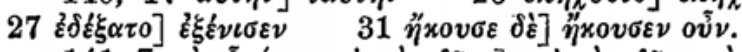

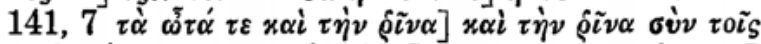

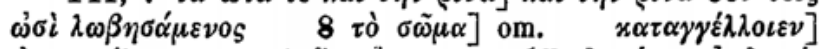

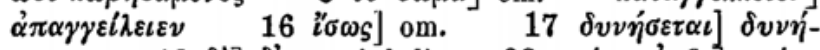

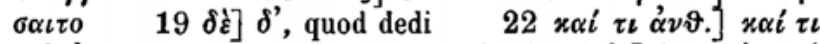
x $i \dot{\alpha} \nu \vartheta$. in verbis vulgo additis $\left.25 \lambda \eta \varphi \vartheta \varepsilon i_{S}\right] \lambda \eta \varphi \vartheta \varepsilon i_{S} x \alpha i$

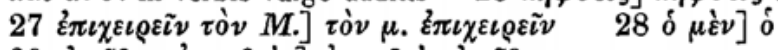

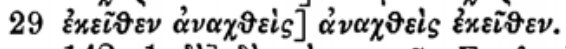

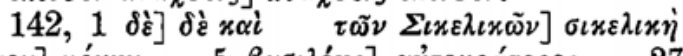

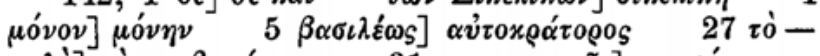

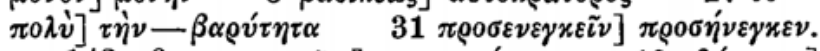

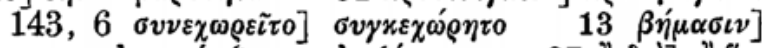

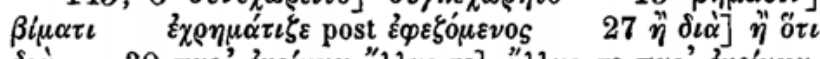

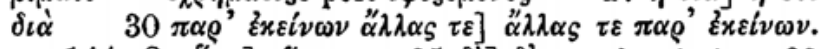

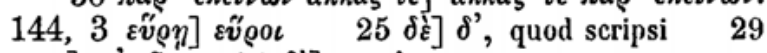
$\alpha \hat{v} \tau \tilde{\omega} \tau \varepsilon] \alpha \hat{v} \tau \tilde{\omega}$ 31 $\delta \dot{\varepsilon}] \gamma \varepsilon \mu \dot{\eta} \nu$.

145,2 हैं

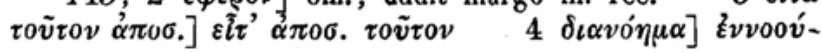
ZONARAS v. 


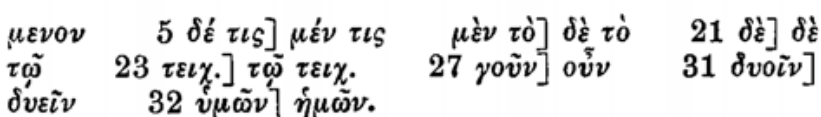

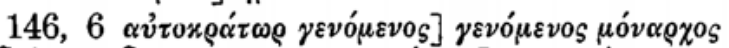

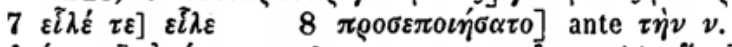

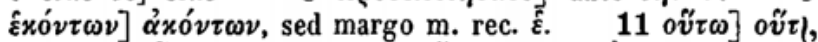

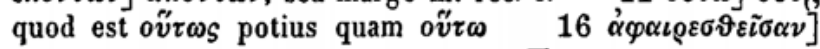

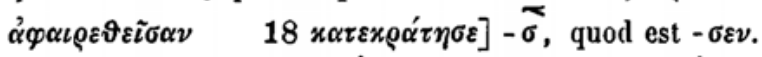

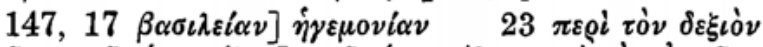

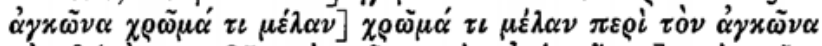

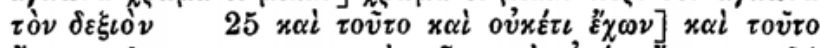

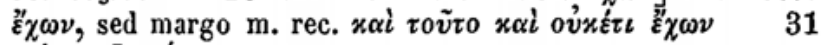
$\pi \alpha \dot{\nu} \tau \omega \varsigma]$ ] $\alpha^{\prime} \nu \tau \varepsilon \varsigma$, sed margo m. rec. $\omega \sigma$.

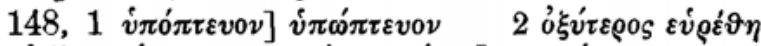

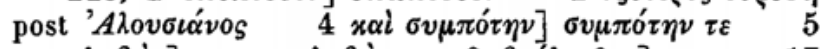

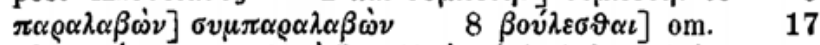

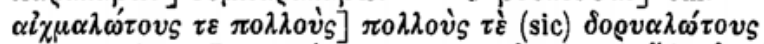

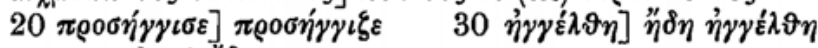

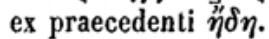

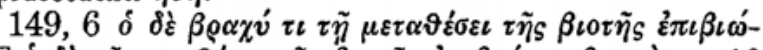

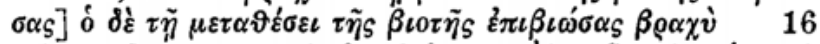

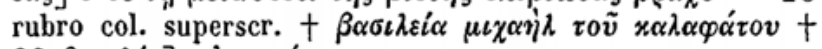

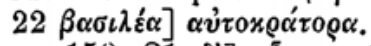

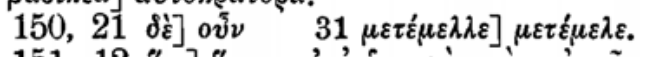

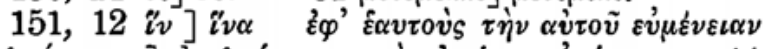

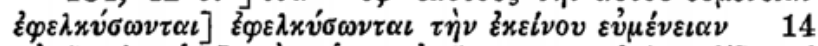

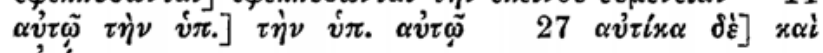

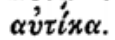

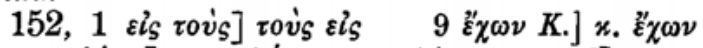

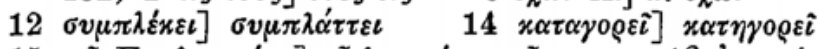

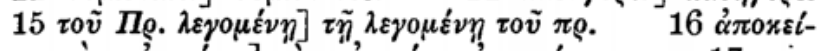

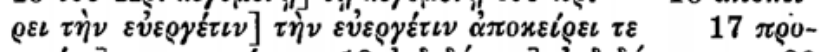

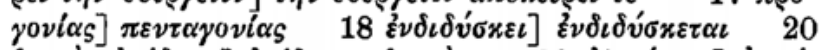

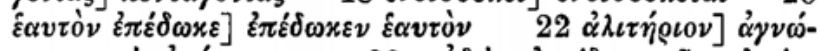

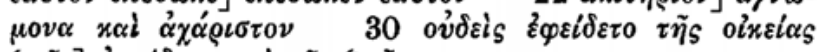

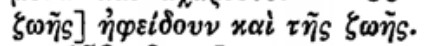

$153,6 \tau \iota]$ pr. $\tau \varepsilon$, ut videtur. Et margo m. rec. $\tau \varepsilon$ 


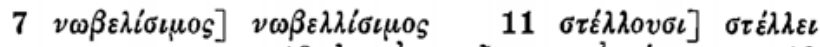

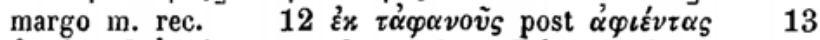

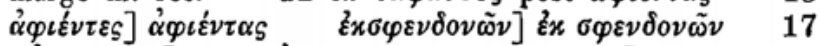

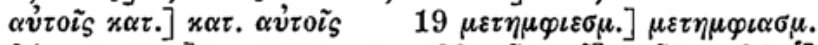

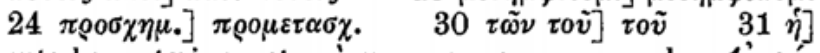
ante hoc asteriscus et $x \alpha i$ marg. m. rec., quum vulgo Avyoú-

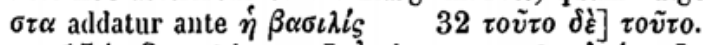

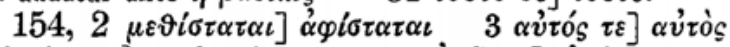
$\left.\nu \omega \beta \varepsilon \lambda i \sigma \iota \mu \circ] \nu \omega \beta \varepsilon \lambda \lambda i \sigma i \mu \sigma_{S} \quad 4 \alpha \pi \tilde{\eta} \lambda \vartheta \varepsilon\right] \dot{\alpha} \pi \tilde{\eta} \lambda \vartheta 0 \nu \quad 6$

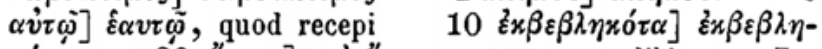

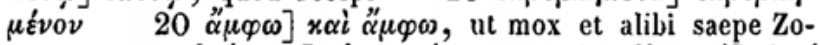

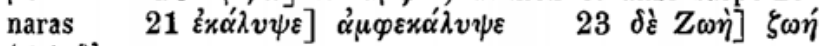
(sic) $\delta$ '.

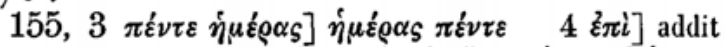

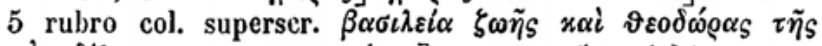

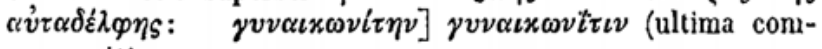

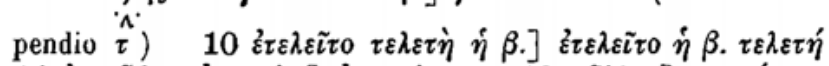

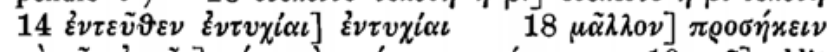

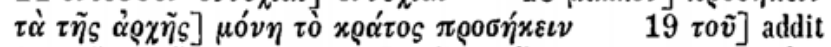

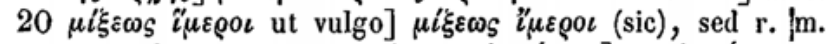

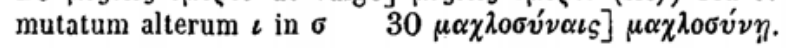

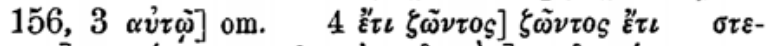

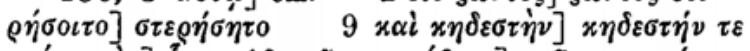

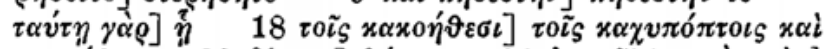

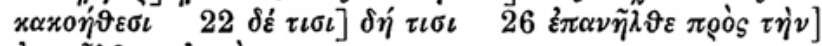
$\varepsilon \pi \alpha \nu \tilde{\eta} \lambda \vartheta \varepsilon \nu \varepsilon \iota_{S} \tau \dot{\eta} \nu$.

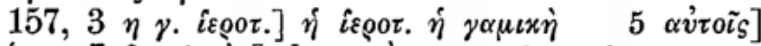

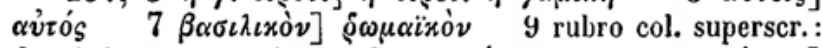

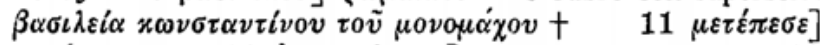

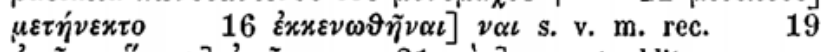
$\stackrel{\alpha}{\alpha} \tilde{\eta} \tilde{\eta} x \nu \stackrel{\alpha}{\alpha} \pi \alpha \sigma \iota] \stackrel{\alpha}{\alpha} \varphi \tilde{\eta} x \varepsilon \nu \quad 21 \tau \dot{\eta} v]$ om. et addit margo m.

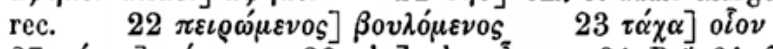

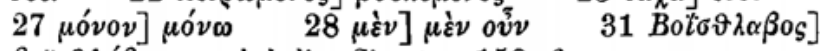

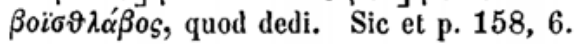

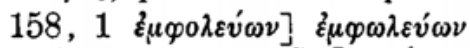
$\gamma^{\prime} \hat{\lambda} \eta$, fere ut A

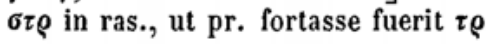

$4 \dot{\eta} \gamma \gamma \dot{\varepsilon} \lambda \vartheta \eta], \dot{\eta} \gamma-$ $11, x \alpha \tau \alpha \sigma \tau \rho \omega \sigma \alpha \varsigma]$ $17 \dot{\alpha} \nu \varepsilon \psi \iota \tilde{\alpha}\rceil \dot{\alpha} \nu \varepsilon \psi \iota \alpha$ 


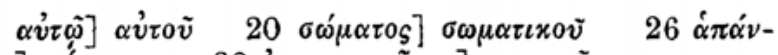

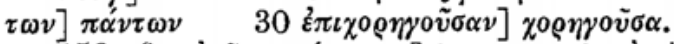

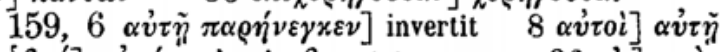

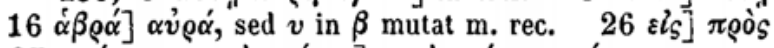

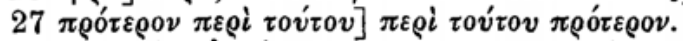

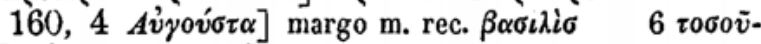
$\tau \circ \nu] \tau \dot{\sigma} \sigma 0 \nu 11 \tau o l]$ om., sed rubro superscriptum ead. $\mathrm{m}$. quae cetera hoc colore scripsit $15 \pi 0 \lambda \varepsilon \mu \eta \dot{\sigma} \sigma \omega \nu] \pi 0 \lambda \varepsilon \mu \eta$ -

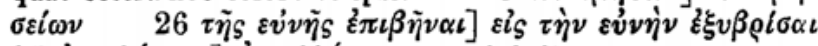
$\left.27 \dot{\alpha} \gamma \gamma \varepsilon \lambda \rho_{\mu} \varepsilon v \alpha\right] \dot{\alpha} \gamma \gamma \varepsilon \lambda \lambda o^{\mu} \mu \varepsilon v \alpha$, quod dedi.

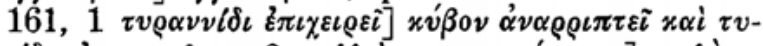

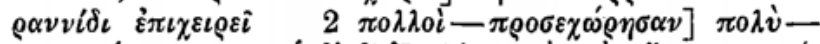

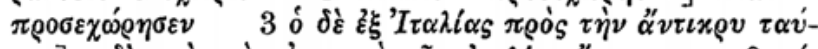

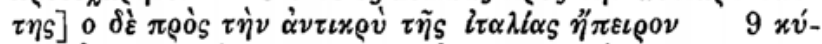

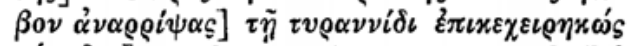

$19 \sigma \tau \varrho \alpha-$ $\tau o ́ \pi \varepsilon \delta o v]$ post hoc asteriscus $\mathrm{m}$. rec., sed nihil in margine

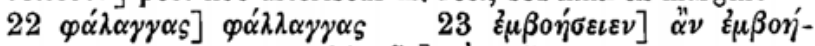

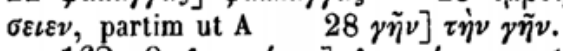

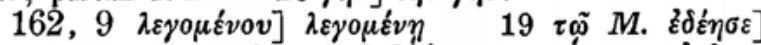

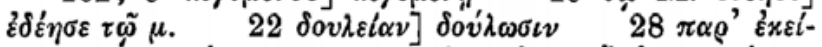

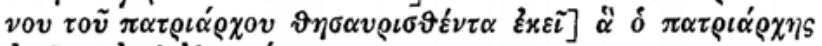

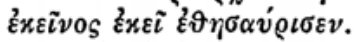

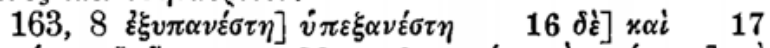

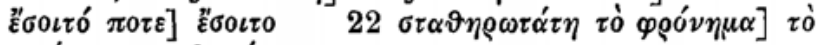

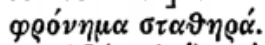

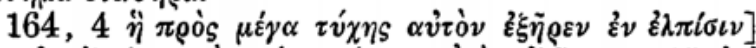

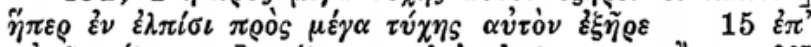

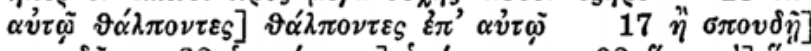

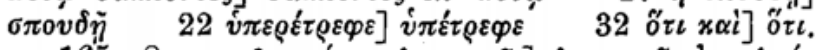

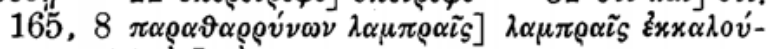
$\mu \varepsilon v 0 \mathrm{~S} \quad 17 \tau \dot{\omega} \nu] \tau \dot{0}$.

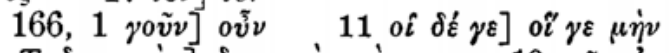

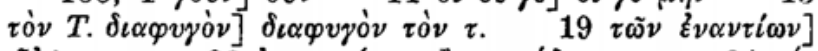

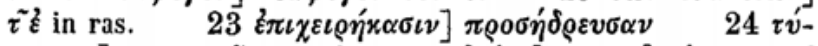

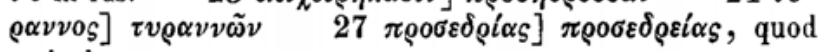
scripsi.

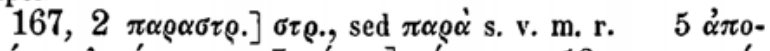

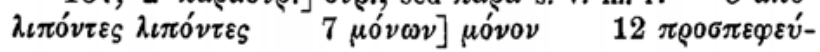




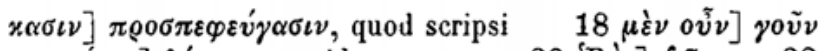

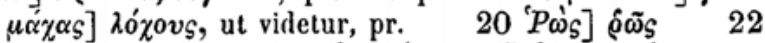

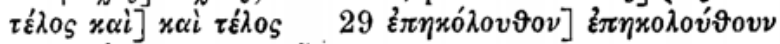
$32 \pi \varrho o ́ \varphi \alpha \sigma \iota \varsigma$ post $\tau 0 \tilde{v} \tau$.

168, 1 б. $\left.\kappa \alpha \vartheta^{\prime} \dot{\eta} \mu \tilde{\omega} \nu\right]$ ] $\alpha \vartheta^{\prime} \dot{\eta} \mu \tilde{\omega} \nu$ б. $\left.10 \tau \varepsilon\right]$ s. v. eal. $\mathrm{m}$. 17 ro่] bis om. $\left.18 \tau \alpha v^{\prime} \alpha \alpha_{\varsigma}\right] \tau \alpha v^{\prime} \tau \iota_{S} \quad 21$ $\left.\left.x \alpha \dot{i} \tau \dot{\eta} \nu] \tau \dot{\eta} \nu \delta \dot{\varepsilon} \quad 25 \delta^{\prime}\right] \delta \dot{\varepsilon} \quad 26 \dot{\alpha} \lambda \hat{\lambda} \dot{\alpha} x \alpha \vartheta \alpha \varrho \dot{\alpha}\right] \dot{\alpha} \lambda \lambda \lambda^{\prime}$

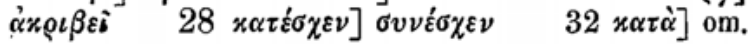

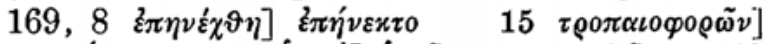

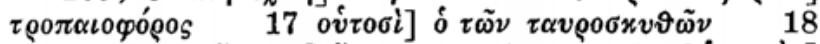

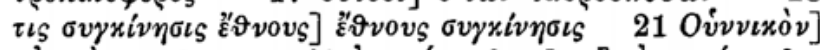

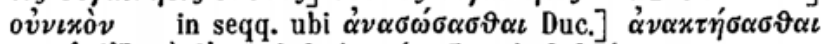

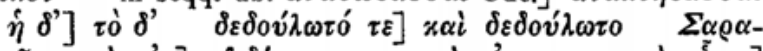

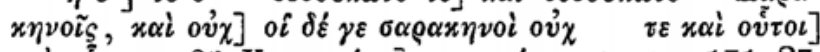

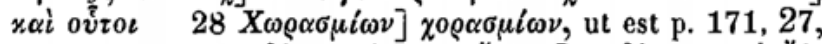

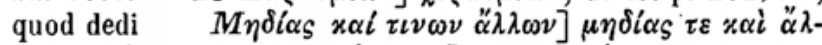

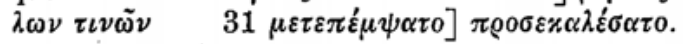

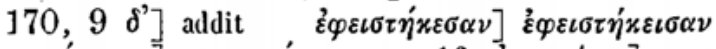

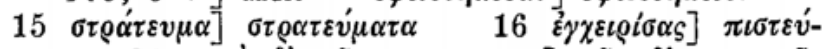

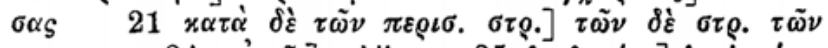

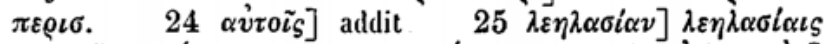

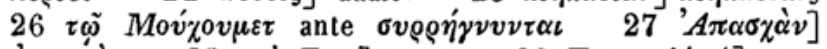

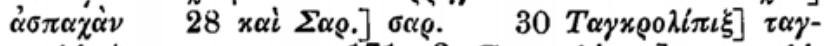

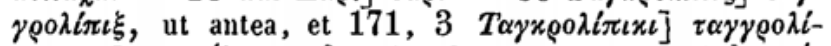

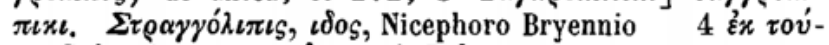

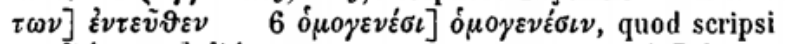

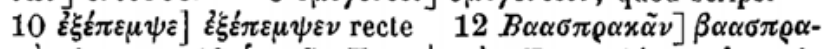

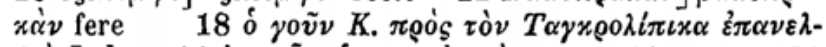

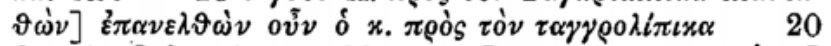

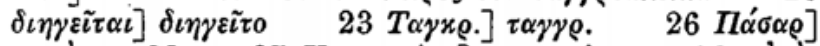

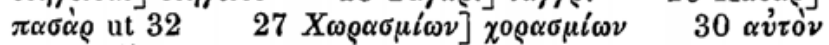
et mox $\delta \dot{\varepsilon}$ om.

$172,4 \pi \tilde{\alpha} \nu] \stackrel{\prime \prime}{\alpha} \pi \alpha \nu \quad 9 B \alpha \alpha \sigma \pi \rho \alpha x \tilde{\alpha} \nu] \beta \alpha \alpha \sigma \pi \rho x \dot{\alpha} \nu$, fere ut A $\left.\left.10 \varepsilon^{\prime} \delta \eta^{\prime} \lambda \omega \sigma \varepsilon\right] \delta \varepsilon \delta \eta^{\prime} \lambda \omega x \varepsilon \quad 19 \varepsilon \iota^{\prime} \pi 0 \iota \mu l\right] \varepsilon \iota^{\prime \prime \pi \omega} \quad 21$

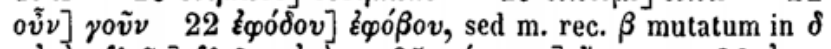

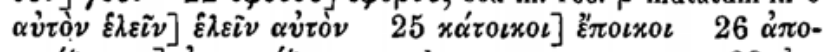

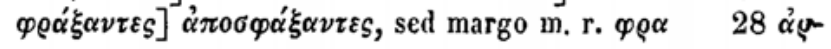




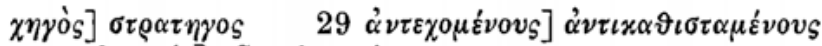

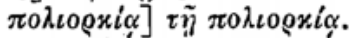

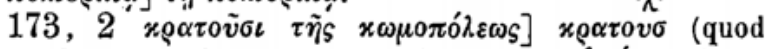
compendium significat $\iota v$, ut paullo post in $\left.\varepsilon \dot{v} \varrho \eta x^{\prime} \alpha \sigma \iota v\right)$ sed

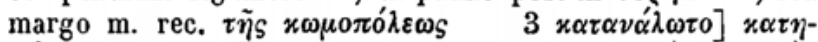

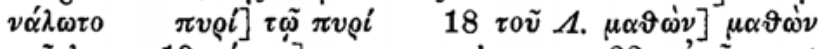

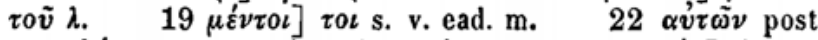

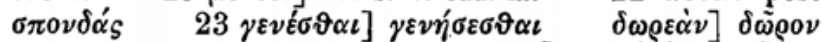

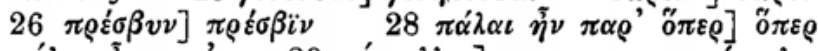

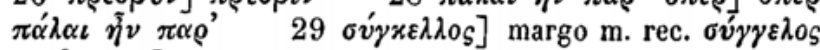
32 ĖxEivos] addit.

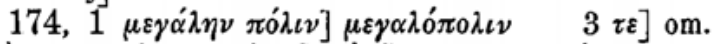

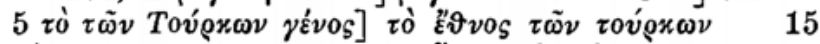

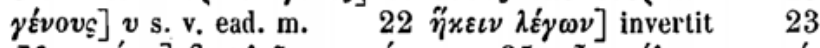

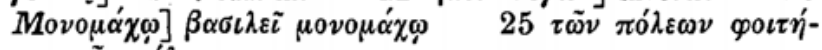
$\sigma \alpha_{S} \tau \tilde{\omega} \nu \pi \hat{\lambda} \varepsilon \omega \nu$.

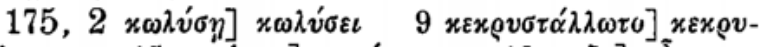

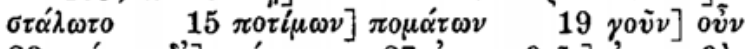

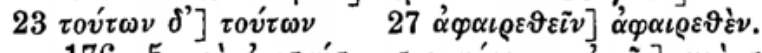

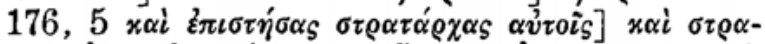

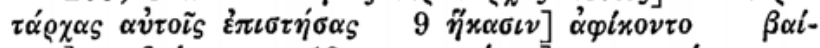

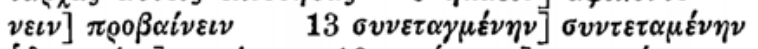

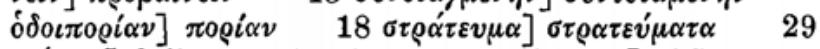
$\left.B o ́ i \lambda \alpha_{S}\right] \beta o i ̈ \lambda \alpha_{S}$, quod scripsi $\left.30 \gamma \lambda \omega \sigma \sigma \sigma \alpha \nu\right] \gamma \lambda \tilde{\tau} \tau \tau \alpha \nu$.

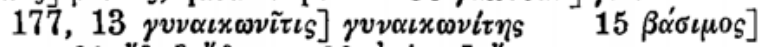

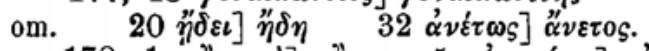

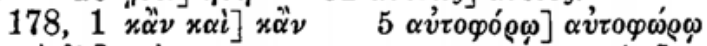

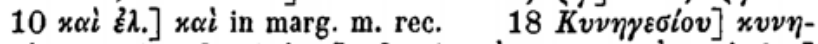

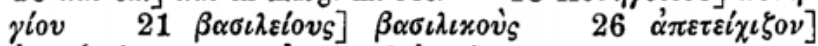

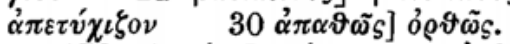

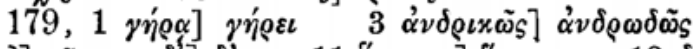

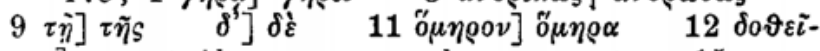
$\sigma \alpha \nu]$ pr., ut videtur, $\tau \iota \nu \alpha$, quod praecesserat $15 \chi \omega \varrho \eta$ -

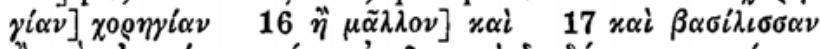

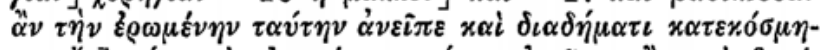

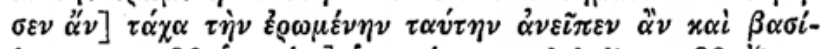
$\lambda \iota \sigma \sigma \alpha \nu 20 \delta \mu \eta \rho i \alpha s] \delta \mu \eta \rho \varepsilon i \alpha s$, quod dedi $23 \dot{\varepsilon} \xi \varepsilon \varepsilon-$

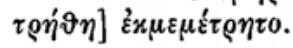




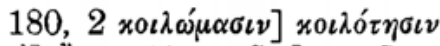

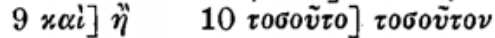
$\left.7 \lambda \varepsilon \dot{\varepsilon}{ }_{0 S}\right] \tau \dot{\varepsilon} \lambda_{O S}$ $11 \mu \eta \tau \tau^{\prime} \alpha \pi \tau \alpha i \sigma \tau \omega_{S}$

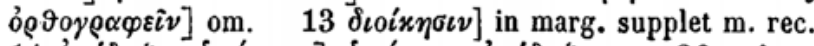

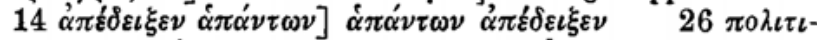

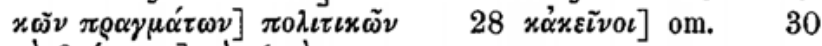
$\tau \dot{0} \beta \iota \omega ́ \sigma \iota \mu \nu] \tau \dot{\eta} \nu \xi \omega \dot{\eta} \nu$.

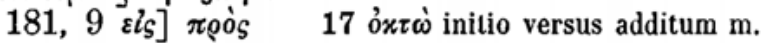

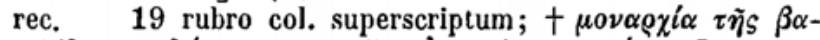

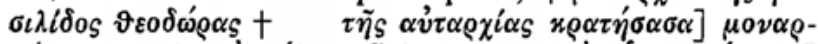

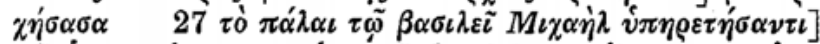

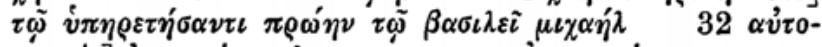

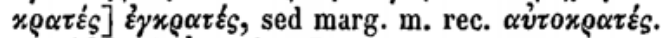

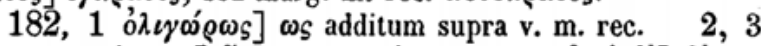
है $v \nu \eta-x \varepsilon x i \nu \eta \nu \tau 0] ~ ह ै \vartheta \nu 0 S-x \varepsilon x i \nu \eta \tau o, 9 \varepsilon$ dit marg. m. rec. $16 \tau \eta \varrho \eta ́ \sigma \varepsilon \iota]$ post $\alpha \dot{v} \tau o \tilde{i}_{S} \quad 19 \mu \varepsilon \tau \alpha-$ $\chi \varepsilon i \varrho \eta \sigma \iota v]$ in summa pagina suppletum $\mathrm{m}$. rec. 21 ö] $\ddot{\eta}$

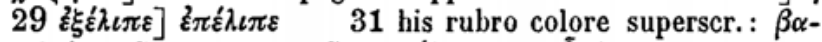
$\sigma \iota \lambda \varepsilon i \alpha \tau \sigma \tilde{v} \sigma \tau \rho \alpha \tau \iota \omega \tau \iota x \circ \tilde{v} \mu \iota \chi \alpha \eta^{\prime} \lambda+\quad$ ovj $\rfloor$ om.

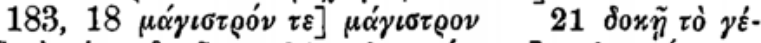

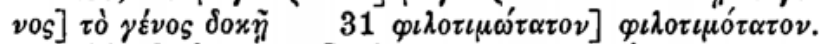

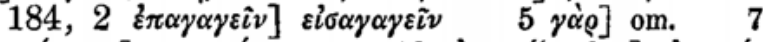

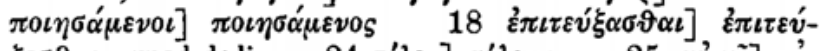

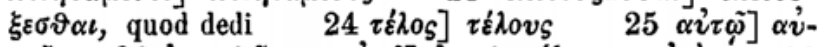

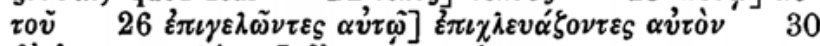

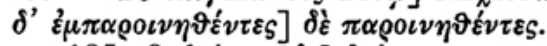

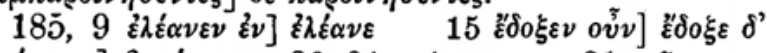

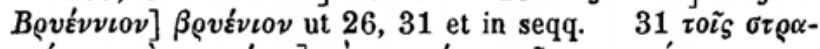

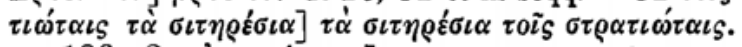

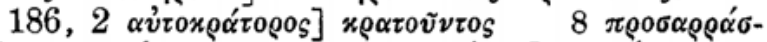

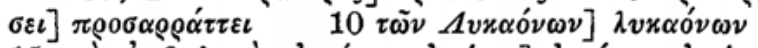

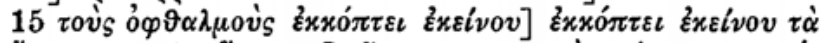

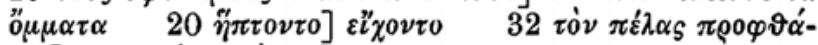

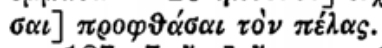

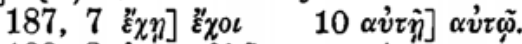

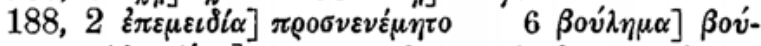
$\left.\lambda \varepsilon v \mu \alpha, 10 \Lambda^{\prime} \omega \nu\right]$ om. cum lacuna eiusdem spatii

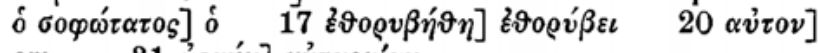
om. $\left.\quad 31 \alpha \varrho \chi \eta^{\prime} v\right] \alpha v i \alpha \varrho \chi^{i} \alpha \nu$. 


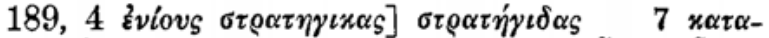

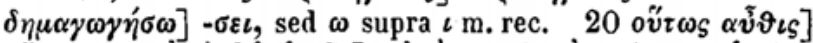

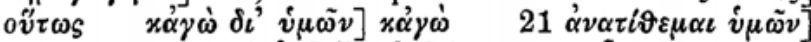

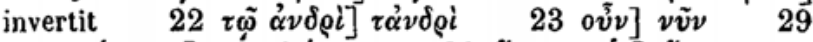

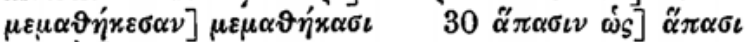
$32 \tau \dot{\eta} \nu]$ addit.

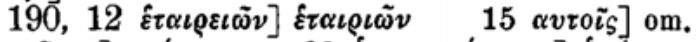

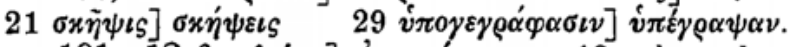

191, $12 \beta \alpha \sigma \iota \lambda \varepsilon i \omega \nu]$ $\alpha \nu \alpha x \tau o ́ \varrho \omega \nu 18$ rubro col. su-

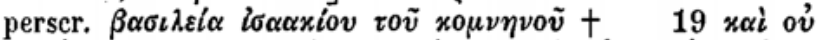

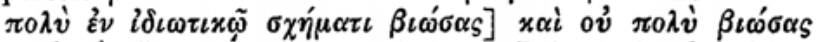
$\mu \varepsilon \tau \dot{\alpha} \tau \dot{\eta} \nu$ हैx $\pi \tau \omega \sigma \iota \nu \quad 24 \beta \alpha \sigma \iota \lambda \varepsilon i \alpha \nu] \beta \alpha \sigma \iota \lambda \varepsilon i \alpha \nu$ है $\chi \alpha \varrho i \sigma \alpha \tau o$

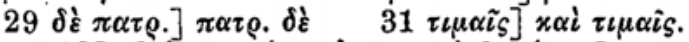

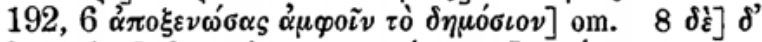

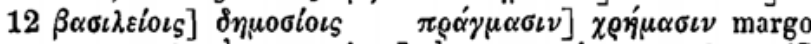

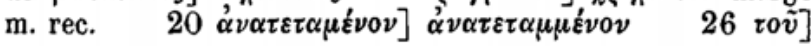
supra v. m. rec.

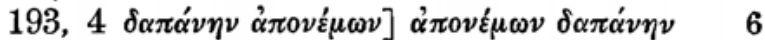

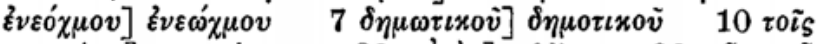

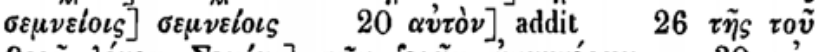

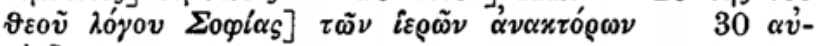
$\tau \dot{\partial} v] \mathrm{om}$.

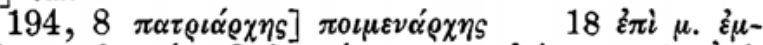

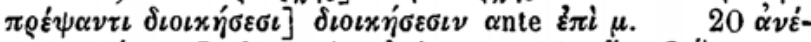

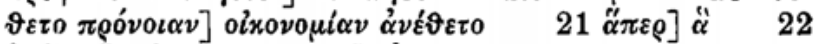

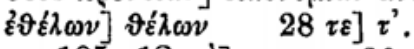

$\left.195,12 \tau \dot{0}] \mathrm{om} . \quad 26 \pi \varepsilon \rho \iota{ }^{\circ} \sigma \alpha \nu \tau \alpha \iota\right] \pi \varepsilon \rho i \sigma \tau \alpha \nu \tau o$ 32 치 om.

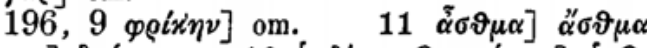

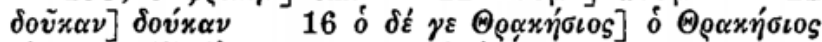

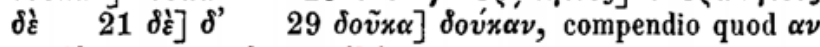
significat scripta altera syllaba.

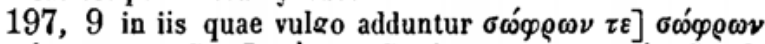

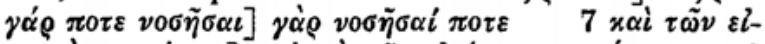

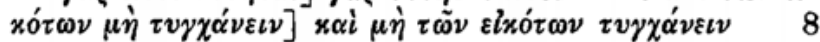

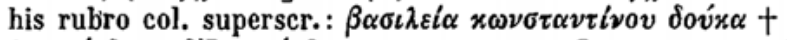

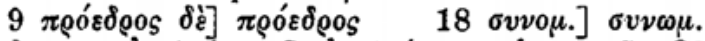

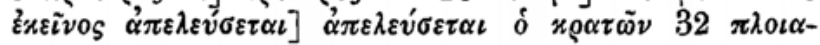


gíov] post hoc dimidium fere versum vacuum relictum, sequente initio proximi $\delta v \sigma_{\varsigma}$, recentior corrector, qui pleraque in margine scripsit, explevit verbis i" $\nu$ ' $\alpha$ vंò $x \alpha \tau \alpha-$

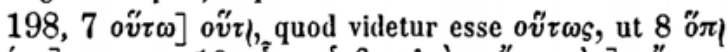
$\left.9 \mu o^{\prime} v \eta\right]$ om. 10 oṽos o $\left.\beta \alpha \sigma \iota \lambda \varepsilon v_{S} \eta v^{\prime} \chi \varepsilon \iota \mu \dot{\varepsilon} v\right] \eta v^{\prime} \chi \varepsilon \iota \mu \dot{\varepsilon} v$

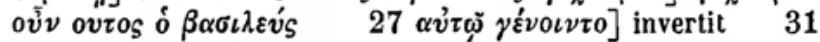

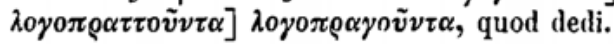

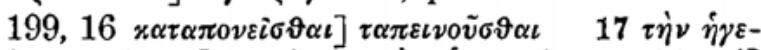
$\mu o v i \alpha \nu \mu \varepsilon \iota 0 \tilde{v} \sigma \vartheta \alpha \iota] \mu \varepsilon \iota 0 \tilde{v} \sigma \vartheta \alpha \iota$ iों $\dot{\eta} \gamma \varepsilon \mu o v i \alpha \nu \quad 24$ xci $]$

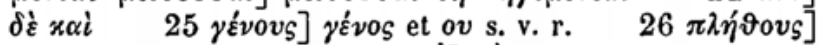
$\pi \lambda \dot{\eta} \vartheta o_{S}$ et $0 v \sigma$ s. v. r. $\left.32 \tau \dot{0}\right] \tau \dot{\nu} \nu$.

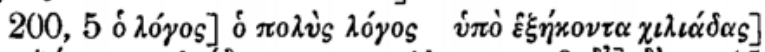

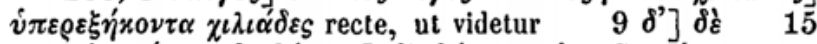

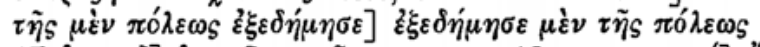

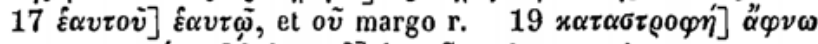

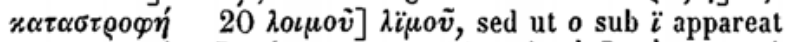

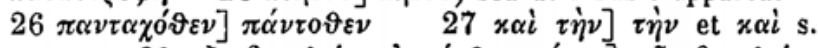

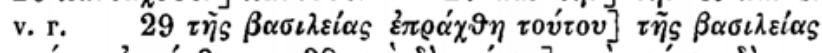

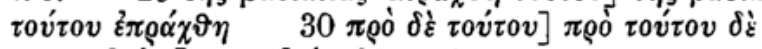

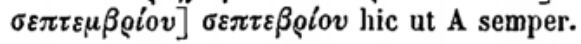

201,1 ह้อ

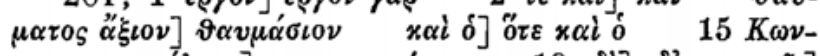

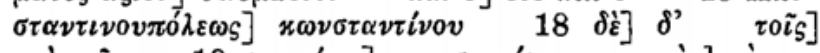

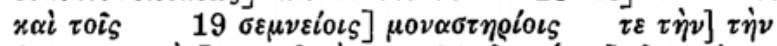

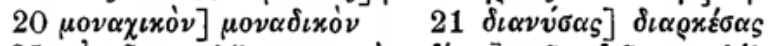

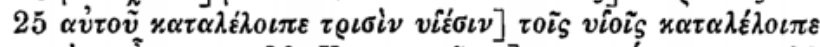

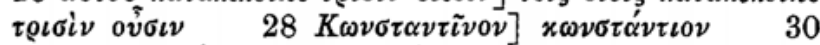

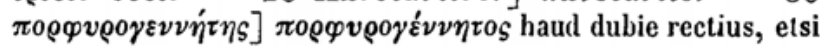
utrumque ponit etiam Ducangius, fingens alterum ex genitivo, qui est p. 198, 16 et alibi. Non minus singulari forma Theodorus Prodr. in Maii Nova Patr. Bibl. vol. 6, part. 2, p. 414,

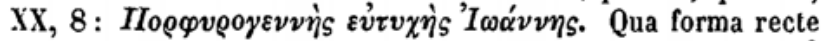
$\nu$ simplici scripta quum infra p. 257, 1 utatur Zonaras: $\Pi \alpha \rho$ '

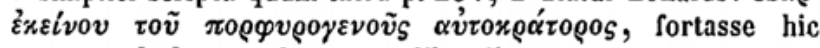
quoque ad alteram aberrarunt librarii.

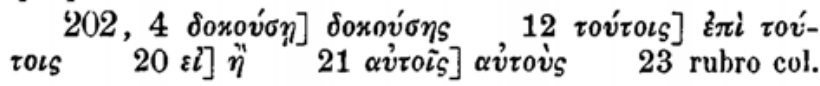




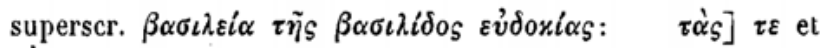
$\tau \dot{\alpha} s$ marg. $\mathrm{r}$.

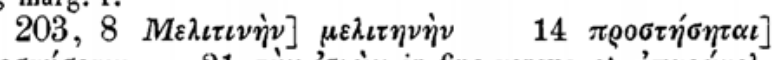

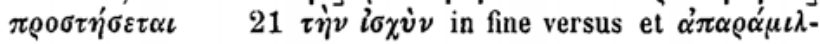
$\lambda o \nu$ initio sequentis eadem manu in marg. scripta $24 \lambda \alpha \mu$ -

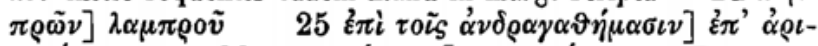

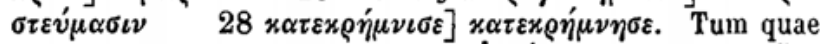
addebantur et sequentia usque ad $\alpha \dot{\xi} \iota \omega \mu \alpha \alpha \iota$ ita exhibet: "í $\nu \alpha$

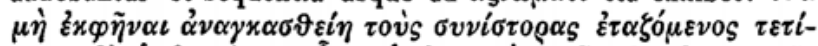

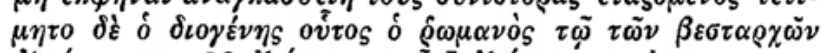

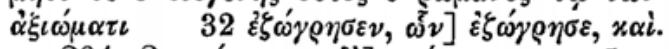

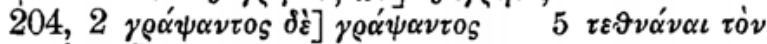
$\alpha v \tau_{0 x \varrho \alpha ́}{ }_{0 \rho \alpha]}$ haec in vacuo spatio quod in codice reliquerat librarius scripsit recentior corrector qui $\tau \varepsilon \vartheta \rho \tilde{\alpha} \nu \alpha \iota$ (sic) in

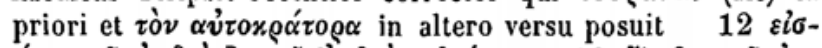

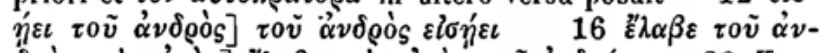

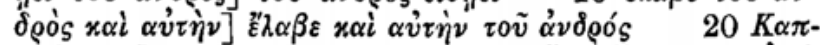

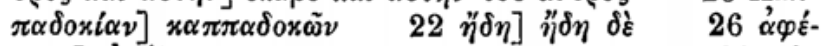
$\lambda \eta \tau 0] \alpha \varphi^{\prime} \varepsilon \eta \tau \alpha \iota$ syllaba $\tau \alpha \iota$ compendio expressa $28 \tau \tilde{\omega} \nu$

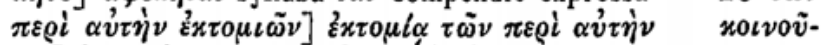

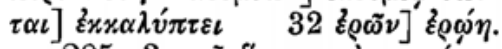

205,3 zoṽ ó

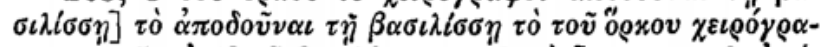

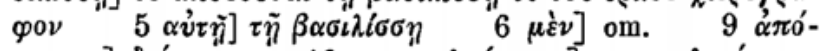

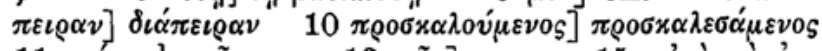

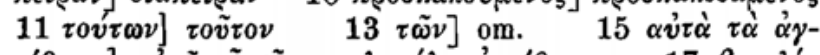

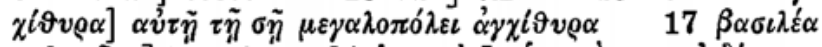

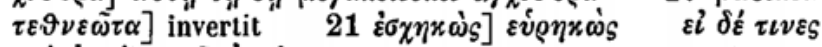
$\left.x \alpha i{ }_{\alpha}^{\alpha} \nu \tau \varepsilon^{\prime} \varepsilon \varepsilon \gamma \sigma \nu\right] \stackrel{\alpha}{\alpha} \tau \dot{\varepsilon} \lambda \varepsilon \gamma \varepsilon$, sed infra versum paginae ultimum

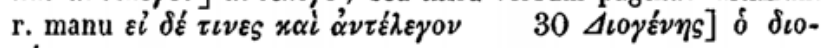

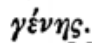

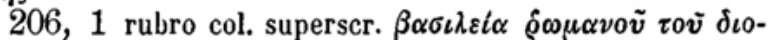

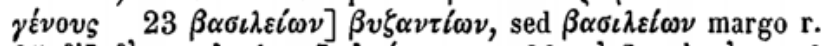

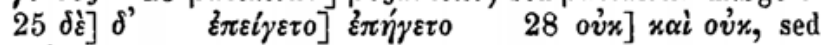
x $\alpha$ i lineola notatum $\mathrm{r}$.

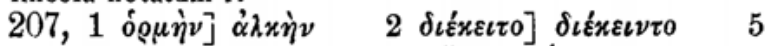
$\left.\tau \tilde{\eta}_{S}\right] \tau 0 \tilde{\iota}_{S}$ pr., $\tau \tilde{\eta}_{S}$ r. $\left.\quad \beta \alpha \varrho \beta \alpha \varrho \iota x \tilde{\eta}_{S}\right] \beta \alpha \varrho \beta \alpha \alpha^{\prime} \iota_{s}$ pr., ex quo $\beta \alpha \rho \beta \alpha \rho \iota x \tilde{\eta} s$ fecit corr. r., qui etiam vacuum in fine paginae 
versus ultimi spatium explevit verbis $\delta \iota \varepsilon \lambda \omega \dot{\nu} \sigma \tau \varrho \alpha \tau \iota \tilde{\alpha}_{S} \quad 11$

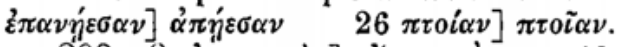

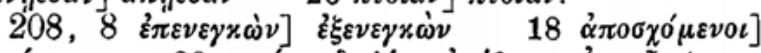

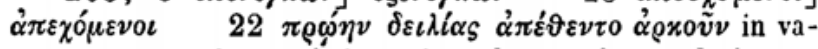
cuo versus paginae primi spatio eodem modo supplevit corrector quo alia paullo ante.

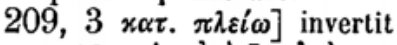

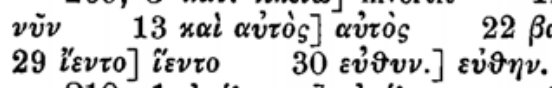

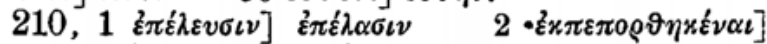

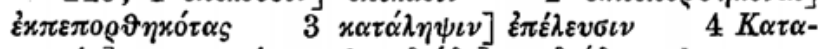

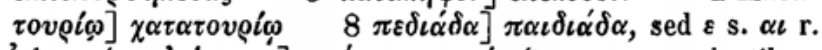

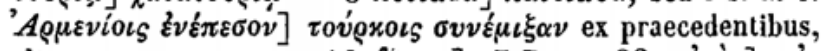

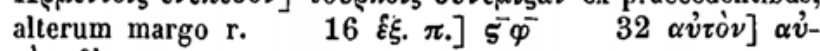

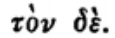

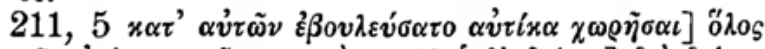

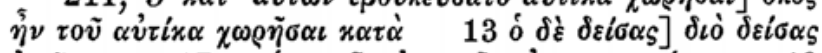

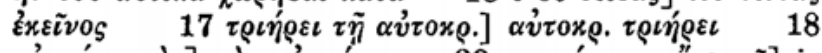

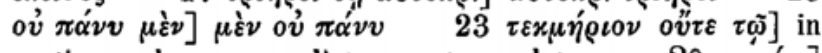
spatio quod vacuum relictum erat supplet r. $29 \pi \rho \circ \eta \eta$ $\varepsilon$ ]

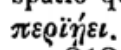

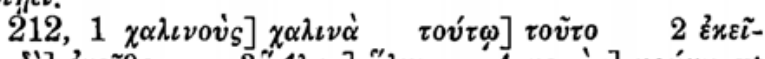

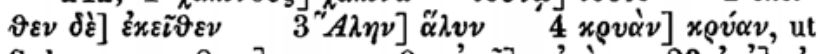

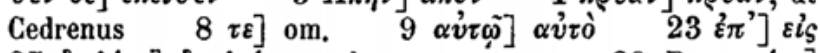

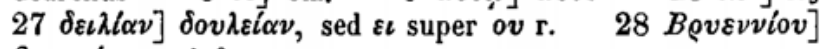

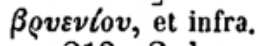

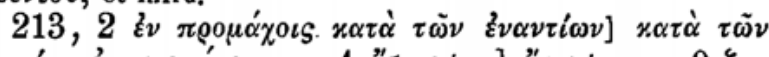

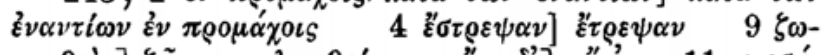


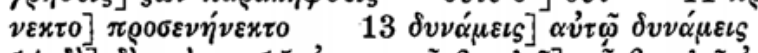

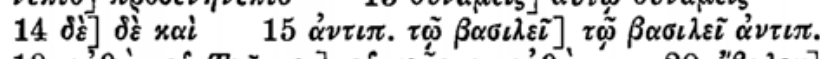

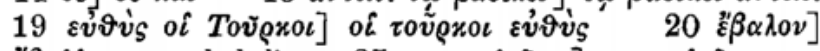

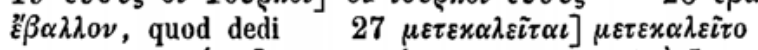

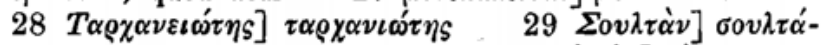

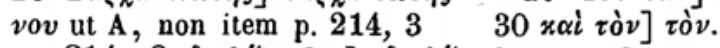

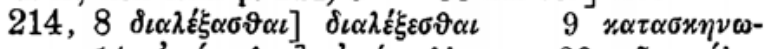

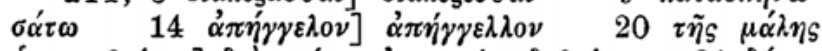

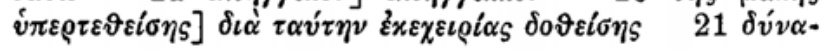




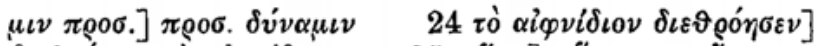

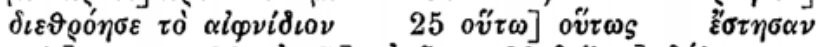

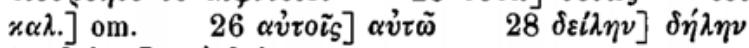

$30 \delta \varepsilon i \sigma \alpha s] x \alpha i \delta \varepsilon i \sigma \alpha \varsigma$.

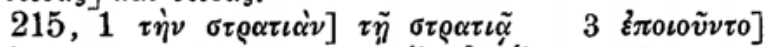

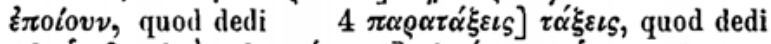

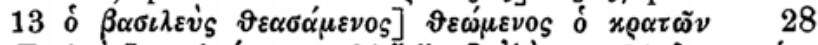

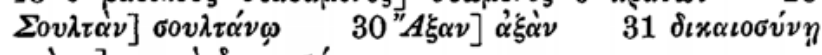

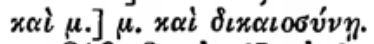

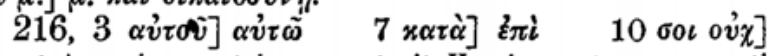
ov $\chi$ ut $\mathrm{A}$, opinor, etsi non notavit Haasius, ut ex sequenti

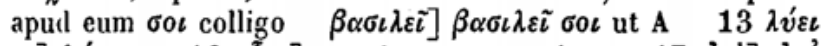

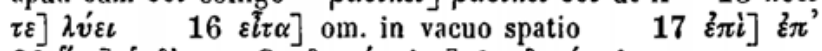

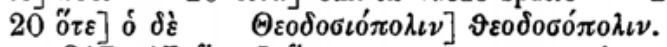

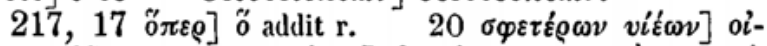

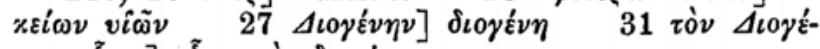

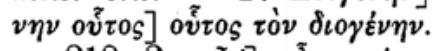

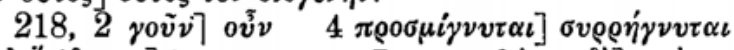

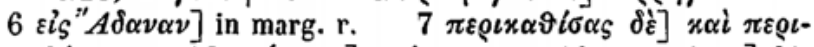

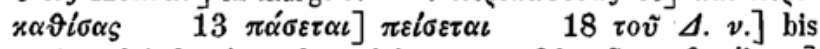
posita, deinde altero loco deleta r. $20 \psi \tilde{\eta} \varphi \circ s \beta \alpha \sigma i \lambda \varepsilon \iota \varsigma$ ]

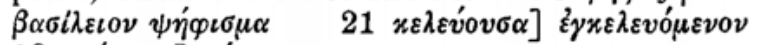

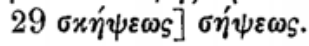

$219,1 \pi \alpha \varrho \dot{\alpha}]$ quum $\stackrel{\varepsilon}{\pi}$ pro $\pi \varepsilon \rho i$ habuisset corr., $\pi \alpha \varrho \dot{\alpha}$

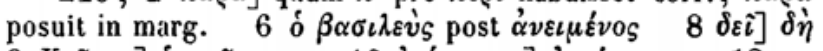

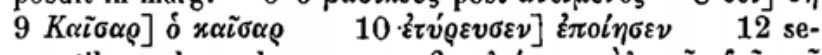

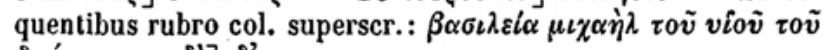

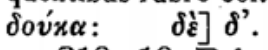

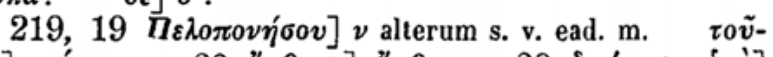

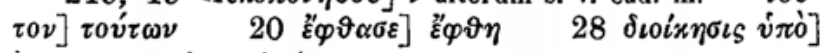
in vacuo spatio scripsit $r$.

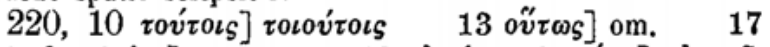

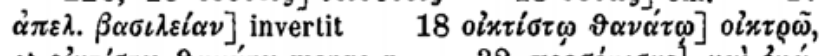

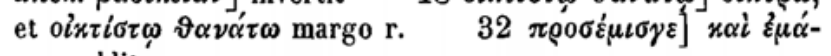
$\chi \varepsilon \tau 0$ addit.

$221,3 \mu \dot{\varepsilon} \nu]$ om. $\quad 10 \stackrel{\alpha}{\alpha} \mu \nu \eta \sigma \tau i \alpha \nu]$ suppletum in

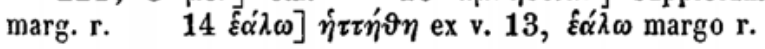




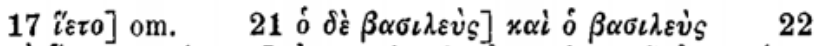

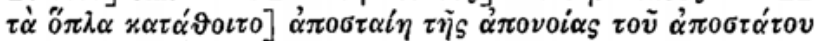
$23 \alpha \dot{v} \tau o \dot{v}] \alpha \dot{v} \tau \tilde{\omega}$, satis commode.

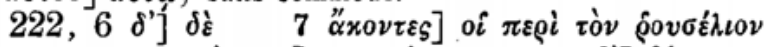

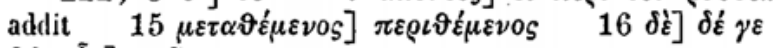

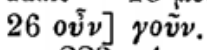

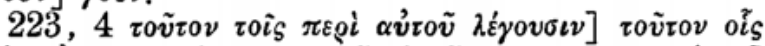

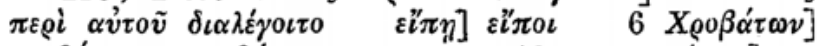
$\chi 0 \varrho \beta \alpha ́ \alpha \omega \nu$ et $\chi \varrho \circ \beta \alpha^{\prime} \tau \omega \nu$ margo r. $\left.10 x \alpha \tau \varepsilon \sigma \chi \eta \mu \varepsilon \dot{\varepsilon} \nu \omega \nu\right] x \alpha-$

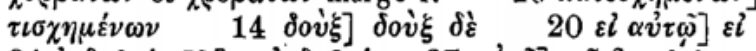

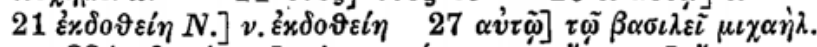

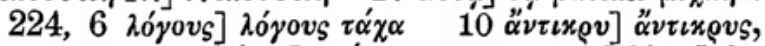

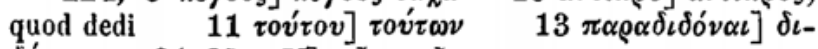
dó $v \alpha \iota$ $24 M-N] \mu \tilde{v}-v \tilde{v}$.

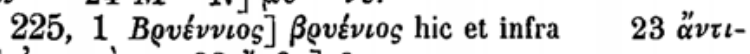
$x \varrho v] \dot{\alpha} v i x \rho \dot{v} \quad 32$ है $\varphi \vartheta \eta] \vartheta$ s. v. r.

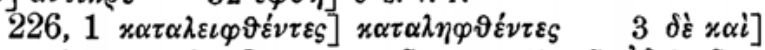
$\begin{array}{lllll}\delta \dot{\varepsilon} & 6 \mu \varepsilon \tau \alpha \sigma \tau \tilde{\eta} \nu \alpha \iota] & \mu \varepsilon \tau \alpha \nu \alpha \sigma \tau \tilde{\eta} \nu \alpha \iota & 7 \tau \tilde{\omega} & \alpha \delta \varepsilon \lambda \varphi \tilde{\omega}-\end{array}$ o $\oint_{0} v$ - omissa in versu paginae secundo in summo margine supplevit corrector r. $13 \pi \rho \circ \chi \varepsilon \varrho i \sigma \vartheta \eta] \pi \varrho 0 \varepsilon \chi \varepsilon \iota i^{\prime}$

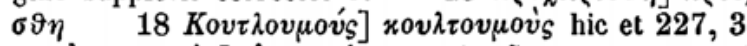
$22 \dot{\alpha} \pi \alpha \gamma \gamma \varepsilon \lambda \vartheta \dot{\varepsilon} v] \dot{\alpha} \gamma \gamma \varepsilon \lambda \vartheta \dot{\varepsilon} \nu$ et $\chi \alpha \lambda_{\iota} \varphi \tilde{\alpha}$ pro Ducangii $\chi \alpha \lambda v \varphi \tilde{\alpha}$, ad quod tacet Haasius $23 \pi \alpha \rho \alpha]$ ] $\pi \alpha \rho$, quod dedi 24

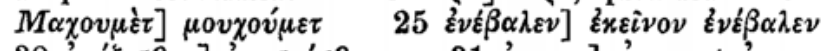

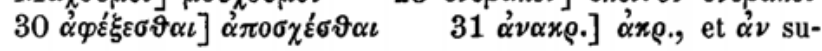
per $\alpha \mathrm{r}$.

227, $6 \pi \varrho \circ v \delta \varepsilon ́ \delta 0 \tau 0] \pi \varrho \circ \delta \varepsilon ́ \delta 0 \tau 0 \quad 7 \pi \alpha \rho \dot{\alpha}] \pi \varepsilon \rho i$ per-

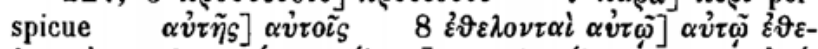

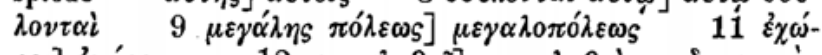

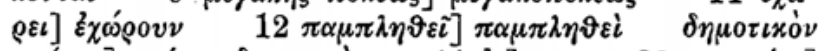

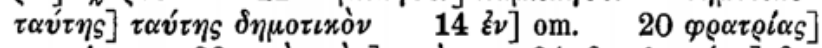

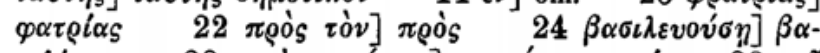

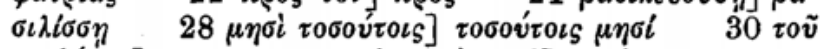

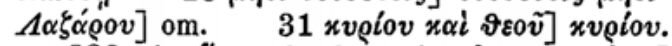

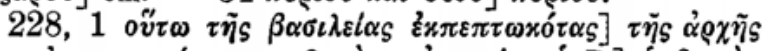

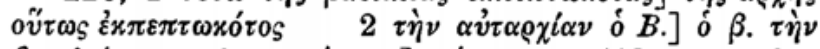
$\left.\beta \alpha \sigma i \lambda \varepsilon i \alpha \nu \quad 9 \pi \rho \circ \varepsilon_{\varepsilon}^{\prime} \mu \psi \alpha_{S}\right] \pi \dot{\varepsilon} \mu \psi \alpha_{S} \quad 12$ sequentibus

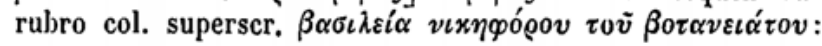




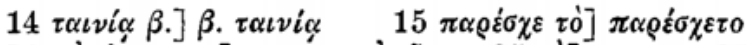

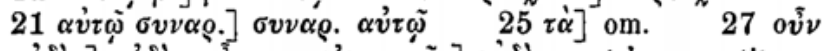

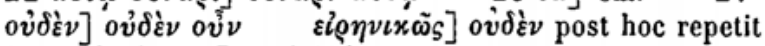
$29 \nu \omega \beta \varepsilon \lambda i \sigma \iota \mu o v] \nu \omega \beta \varepsilon \lambda \lambda i \sigma \iota \mu o v$.

229, $2 \pi 0 \lambda \lambda \tilde{\omega} \nu \tau \varepsilon x \alpha i \stackrel{\alpha}{\alpha} \alpha \vartheta \tilde{\omega} \nu] \pi 0 \lambda \lambda \alpha \tilde{\iota} \tau \varepsilon x \alpha i \dot{\alpha} \gamma \alpha-$

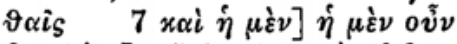
$9 x \alpha i$ oi $B . x \alpha \tau \dot{\alpha}$ iov

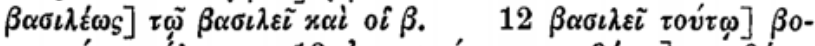

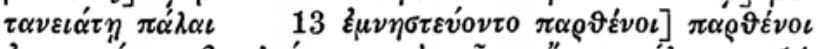

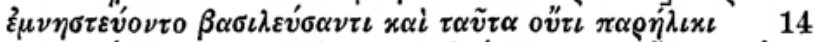

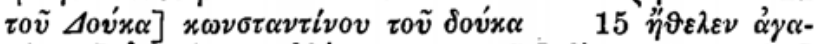

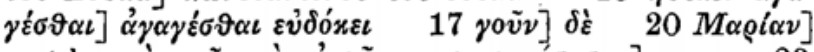

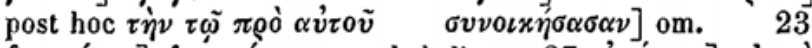

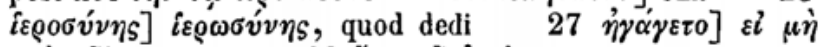

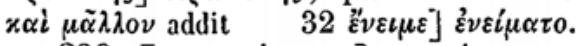

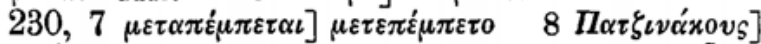

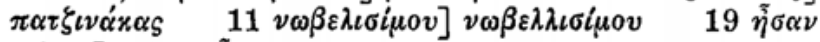
$\left.\chi \varepsilon \tilde{\imath} \varrho \alpha_{S}\right] \chi \varepsilon \hat{\imath} \varrho \alpha \varsigma \tilde{\eta} \sigma \alpha \nu$.

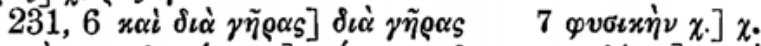

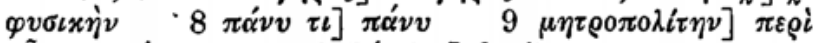

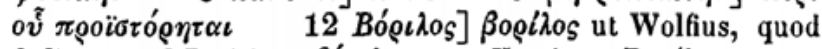
dedi, etsi ad Parisinae $\beta o ́ \varrho \iota \lambda o_{S}$ tacet Haasius. Bo@i $\alpha \nu \nu$ omnes p. $228,9, B 0 \varrho i \lambda \alpha_{S}$ Niceph. Bryennius p. 97, C; 98, A. In-

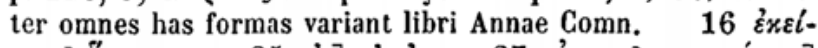

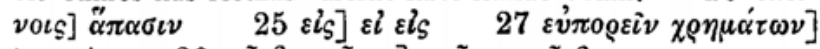
invertit $28 \tau \tilde{\tau} \beta$. $\chi \varrho \tilde{\eta} v \alpha \iota] \chi \varrho \tilde{\eta} \nu \alpha \iota \tau \tilde{\omega} \beta$.

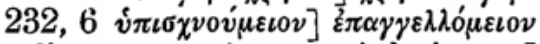

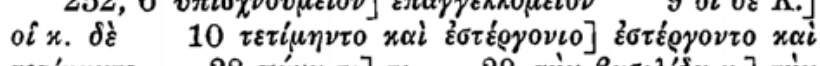

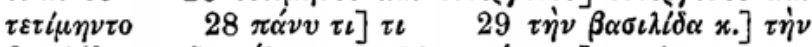

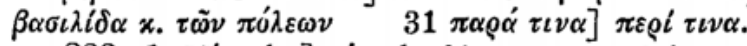

233, $\left.\left.1 N k^{\prime} \mu \varepsilon \tau \xi o \iota\right] v \varepsilon \varepsilon \mu \tau\right\} \circ \iota$ bis, ut omnes infra et supra 212,5 . Itaque hic quoque praetuli, quod est etiam apud Annam Comn. p. $\left.\left.62, \mathrm{~B} ; 63, \mathrm{C} \quad \delta^{\prime} \circ i\right] \delta \eta^{\prime} \quad 6 \alpha^{\prime \prime} v \tau \iota x \rho v\right]$

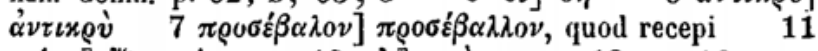

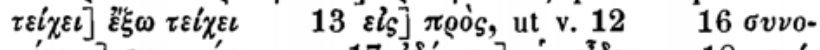

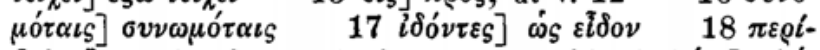
$\beta 0 \lambda o v]$ ante hoc lacuna eiusdem spatii $\left.20 \pi \lambda \eta \vartheta v^{\prime} s\right] \pi \lambda \dot{\eta}$ จovs, ovs per compendium, sed acc. super $\eta^{\prime}$.

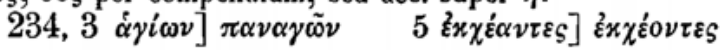




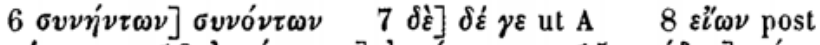

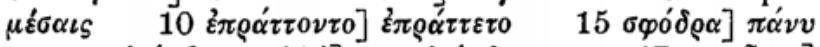

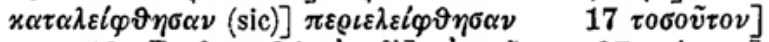

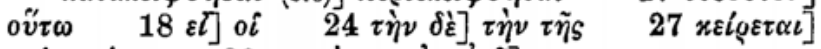

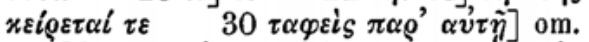

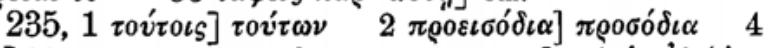
$\eta ౌ \delta \eta]$ his et seqq. rubro col. superscriptum: $\beta \alpha \sigma \iota \lambda \varepsilon i \alpha \dot{\alpha} \lambda \varepsilon \xi \xi i o v$

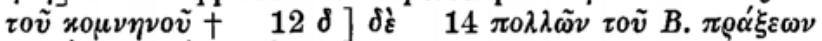

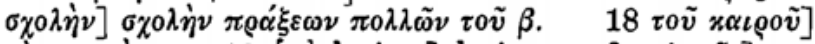

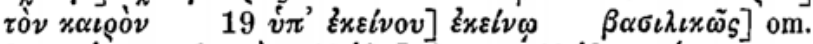

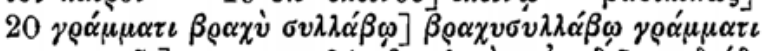

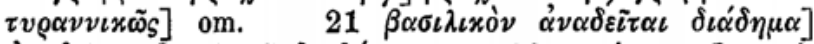

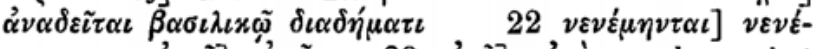

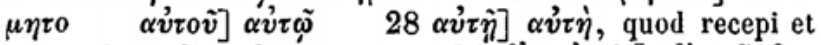
vix credam aliter legí in A $\left.31 \delta^{\prime} \alpha v \tau o \tilde{c} s\right] \quad \delta \dot{\varepsilon} \tau \tilde{\omega} \beta \alpha-$ $\sigma \iota \lambda \varepsilon \hat{i}$.

236, $11 \sigma v \mu \beta \iota \beta \alpha ́ \sigma \varepsilon \iota] \sigma v \mu \beta \alpha \dot{\sigma} \sigma \iota \quad 12 \Theta \varepsilon \sigma \sigma \alpha \lambda o v i \varkappa \eta \nu$

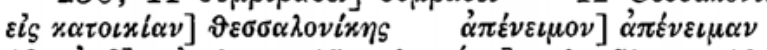
$\left.13 \alpha \dot{v} \tau \tilde{\omega}] \alpha \hat{v} \tau 0 \hat{\imath} S \quad 15 \tau \eta \lambda \iota x \circ v^{\prime} \omega\right] \tau \eta \lambda \iota x \tilde{\omega} \delta \varepsilon \quad 18 \tau \alpha-$

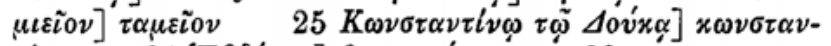

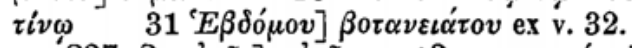

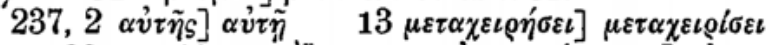

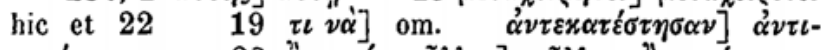

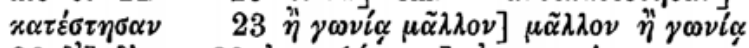

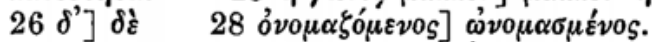

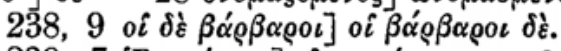

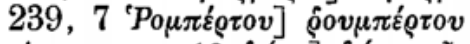
ह่ $\pi \alpha v i \sigma \tau \alpha \nu \tau 0$ oṽ̃os

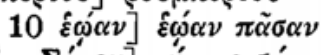

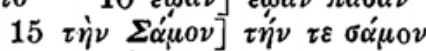
$9 \varepsilon \pi \alpha \nu i \sigma \tau \alpha \nu \tau \alpha \iota]$ $11 x \alpha i$ ov $\left.{ }^{2} \sigma s\right]$ $23 \varepsilon \pi \alpha v \varepsilon \sigma \omega ́-$

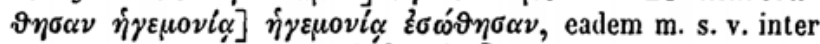

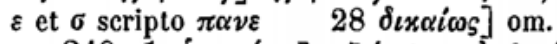

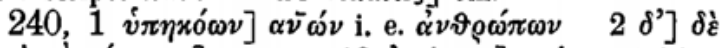

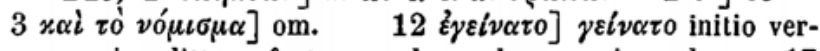
sus, omissa littera fortasse rubro colore appingenda

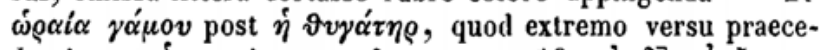
denti post $\dot{\omega}_{S}$ ascriptum eadem m. $\left.19 \alpha \dot{v} \tau \hat{\omega}\right] \alpha \dot{v} \tilde{\eta}$ $20 \tau \tilde{\eta}]$ om. $\left.22 x \alpha \varepsilon \varepsilon \gamma \gamma v \eta^{\prime} \sigma \alpha_{S}\right] x \alpha \tau \eta \gamma \gamma v \eta^{\prime} \alpha_{S} \quad 25 x \alpha \tau$.

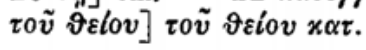




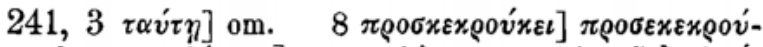

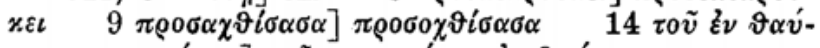

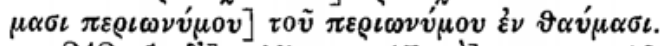

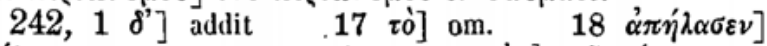

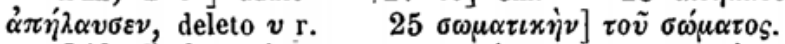

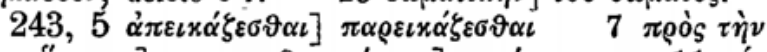

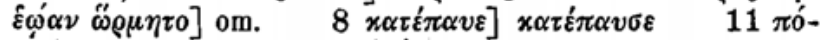
$\left.\lambda i v] \pi 0 \lambda_{\iota 0 \rho x i \alpha \nu} 25 \alpha \dot{v} \tau \dot{\alpha}\right] \tau \alpha \tilde{v} \tau \alpha$.

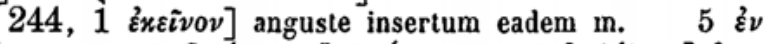

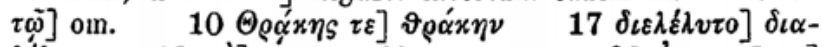
$\left.\lambda_{\varepsilon}^{\prime} \varepsilon_{v \tau o} 18 \mu \eta\right]^{\prime}$ om. et addit margo r. $23 \dot{\alpha} \nu \alpha \iota x$. Duc.]

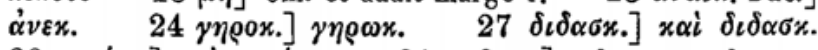

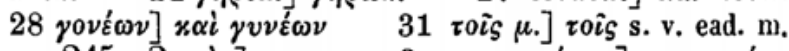

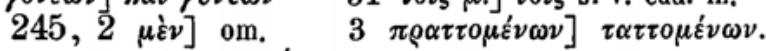
Alioqui exspectes $\pi \varrho \alpha \tau \tau o ́ v \tau \omega \nu$. Alterum infra, sed alia con-

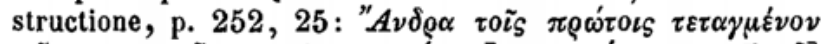

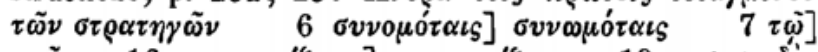

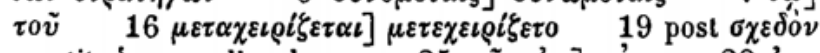

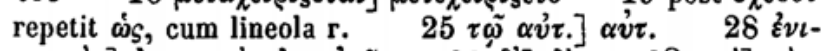

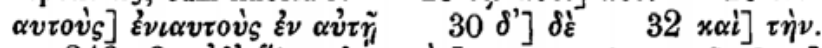

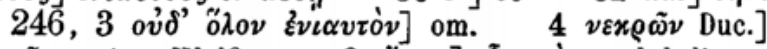

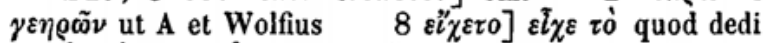

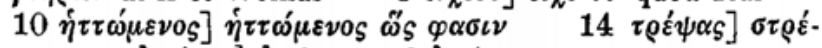

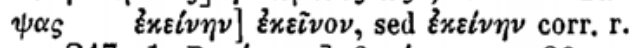

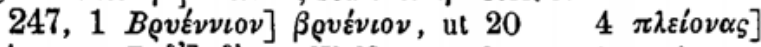
$\left.\pi \lambda \varepsilon i o v s \quad 7 \delta^{\prime}\right] \delta \dot{\varepsilon}$, ut Wolfius, et fortasse A, etsi tacet Haasius $13 \sigma \chi \varepsilon \delta \delta \dot{~} v$, post $\left.\sigma \dot{v} \mu \pi \alpha \nu \tau \alpha S \quad 15 \delta^{\prime}\right] \delta \dot{\varepsilon}$, $28 \mu \varepsilon \tau \dot{\alpha} \tau \tilde{\omega} \nu] \mu \varepsilon \tau$ ' $\alpha \dot{v} \tau \tilde{\omega} \nu$ pr. eadem m. corr. $\alpha \dot{v} \tau \dot{v} v]$

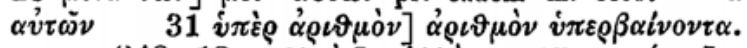

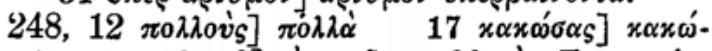

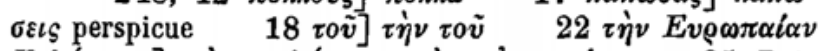

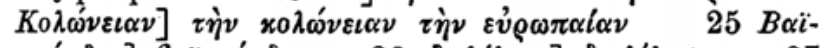

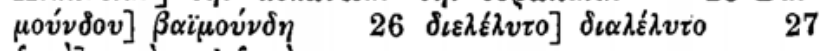
$\hat{\varepsilon} \pi \tau \dot{\alpha}] \mu \varepsilon \tau \dot{\alpha}$, sed $\dot{\varepsilon} \pi \tau \alpha$ margo r.

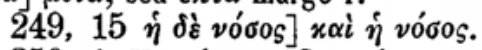

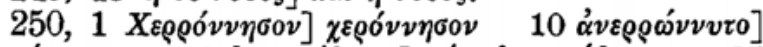

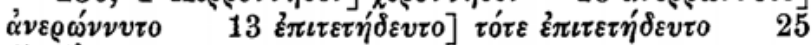

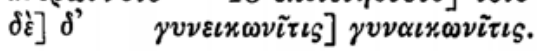


251, $2 \Pi \alpha v \lambda \iota x \iota \alpha \dot{\nu} 0 v \varsigma] \pi \alpha v \lambda \iota x \iota \alpha \nu 0 \dot{v} \varsigma \quad 4 T \xi \iota \mu \iota x \dot{\eta}]$

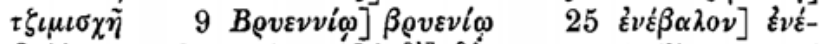
$\beta \alpha \lambda \lambda_{0 \nu}$, quod recepi $\left.26 \delta \dot{\varepsilon}\right] \delta \varepsilon \dot{\varepsilon} \gamma \varepsilon$ saepe sic illatum apud Zonaram, ut p. 252, 6, ubi $\delta \dot{\varepsilon}$ A.

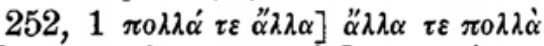

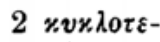

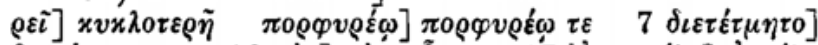

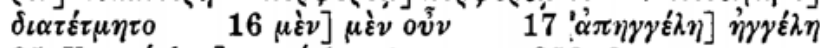
$\left.25 K \alpha \mu \mu v^{\prime} \zeta \eta v\right] \varkappa \alpha \mu v^{\prime} \zeta \eta v$, iterumque $253,3$.

253, 2 है $\varphi \varepsilon v \gamma o v]$ है $\varphi v \gamma o v$, quod recepi $\left.15 \delta^{\prime}\right] \delta \dot{\varepsilon}$ $\left.21 \pi 0 \lambda \varepsilon \mu i \iota_{\zeta}\right] \pi 0 \lambda \varepsilon \dot{\mu} \omega$

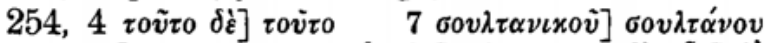

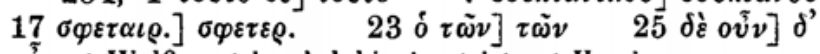
ov $\nu$ ut Wolfius et haud dubie A, etsi tacet Haasius.

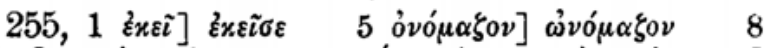

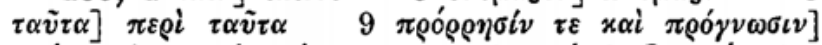

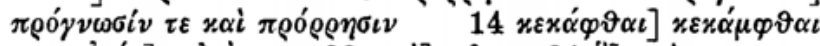

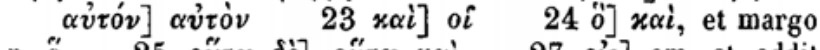

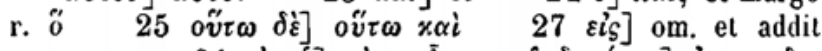

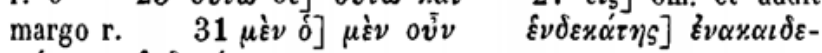

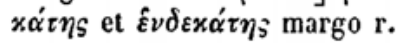

$\left.256,1 \stackrel{\tilde{\alpha} \sigma \vartheta \mu \alpha]}{\alpha} \sigma \vartheta \mu \alpha \quad 2 \beta \alpha \sigma i \lambda_{\iota} \sigma \sigma \alpha\right] \beta \alpha \sigma \iota \lambda$ is $\quad 7$

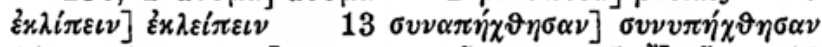

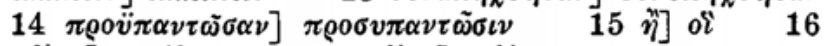
$\left.v i \varepsilon ́ \omega \nu] \pi \alpha i \delta \omega \nu \quad 20 v i \hat{i}^{\prime} \omega s\right]$ ví́os, quod recepi

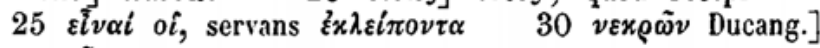


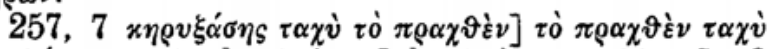

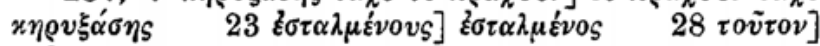
roṽro, quod dedi.

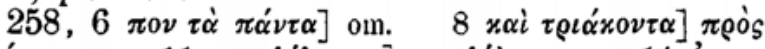

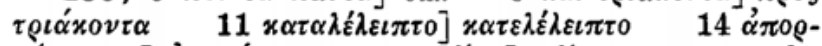

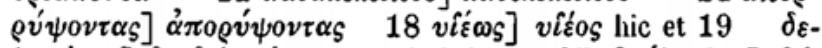

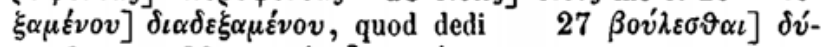

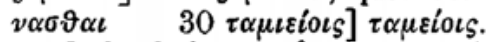

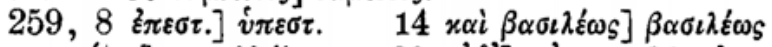
$\left.22 \mu \varepsilon \tau \alpha \tau \alpha^{\prime} \xi \alpha \iota\right] \mu \varepsilon \tau \alpha \lambda \lambda \alpha^{\prime} \xi \alpha \iota \quad 23$ ov่d'] ov 24 oino-

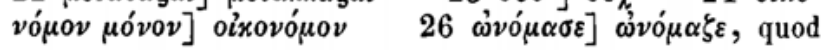
ZONARAS V. 
CXLV I

AD VOL. IV.

dedi $29 \tau \alpha \pi \varepsilon \imath \nu \tilde{\omega} \sigma \alpha \iota]$ s. v. suppletum r. $\quad 30 \stackrel{\alpha}{\alpha} \pi \alpha \sigma \iota]$

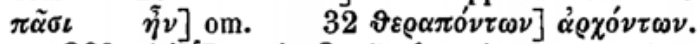

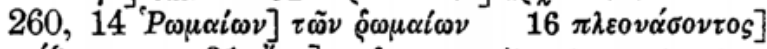

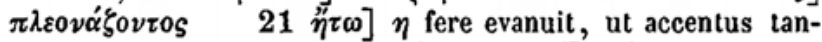
tum apices appareant $23 \lambda \varepsilon i \pi 0 \nu \tau \alpha \nu v \nu i] \lambda \varepsilon i \pi \sigma \nu \tau \tau \alpha \nu \tilde{v} \nu$ $26 \quad \delta \dot{\varepsilon}] \delta^{\prime}$. 